\title{
Design Principles for Interface Thermal Conductance
}

A Dissertation
Presented to
the Faculty of the School of Engineering and Applied Science
University of Virginia
In Partial Fulfillment
of the requirements for the Degree
Doctor of Philosophy (Electrical and Computer Engineering)
by

Carlos A. Polanco

November 2015 


\section{Approval Sheet}

This dissertation is submitted in partial fulfillment of the requirements for the degree of

Doctor of Philosophy (Electrical and Computer Engineering)

Carlos A. Polanco

This dissertation has been read and approved by the Examining Committee:

Avik Ghosh, Adviser

Pamela M. Norris, Committee Member

Patrick E. Hopkins, Committee Member

Joseph Poon, Committee Member

Lloyd R. Harriott, Committee Chair

Accepted for the School of Engineering and Applied Science:

Dean, School of Engineering and Applied Science

November 2015 


\section{Abstract}

The performance, reliability and lifetime for a system is often closely related to its temperature of operation. This temperature depends on the thermal energy generated within the system and on the thermal resistance, which determines how easy the energy flows to the external world. As the size of a system decreases to the nanoscale (on the order of tens of nanometers), its thermal resistance is dominated by interfaces. Thus, one way to engineer the operation temperature of a "small" system is to tune the resistance at its interfaces. This tuning process can be done by changing interfacial properties known to affect the value of resistance such as interatomic mixing or roughness.

Inspired by concepts on electronic impedance matching and photonic antireflection coating, we study the fundamental principles and design rules that determine the interface thermal resistance. For our particular systems, heat is carried by a broadband spectrum of interacting phonons instead of a single frequency non interacting electronic or photonic wave. In this dissertation, we focus on interfaces between two crystalline solids joined by a variable thin intermediate layer. We explain how to maximize thermal conductance on 1D atomic chains and 3D crystalline solids, with intermediate layers varying from a single atom to graded junctions, in the coherent, incoherent elastic and incoherent inelastic regimes. We also explain the role of interatomic mixing and crystal structure on the interface conductance. Our theory is built from a Landauer description of conductance that highlights the interplay between the number of propagating channels available for conduction and the average transmission per channel. Rigorous simulations using non-equilibrium Green's function formalism (NEGF), coupled with known interatomic potentials or first principles parameters, support our results. We also compare our NEGF results with non-equilibrium molecular dynamics simulations. 


\section{Acknowledgments}

The work presented in this document has been possible because of the support of many people. I want to express my gratitude to my advisor, Professor Avik Ghosh, whose influence has shaped me into the researcher I'm today. He has taught me to think simple, to make equations talk, to work in baby steps, to make effective presentations and many more important skills to do research. He has been very patient with my stubbornness and has given me advice beyond the academic life. I also want to thank the members of my committee, Professors Lloyd R. Harriott and Joseph Poon and specially Professors Pamela Norris and Patrick Hopkins for mentoring and guiding me along the field of Nano scale heat transfer. I want to thank Professor Nathan Swami, who was my advisor during my first year. He gave me an opportunity that changed my life.

I want to thank the members of my research group, and the members from Professors Norris and Hopkins groups as well, for their friendship and all the innumerable enlightening meetings. I want to thank Jingjie

Zhang and Rouzbeh Rastgar for their time, interest and collaboration with first principles parameters and MD simulations.

Last but not least, I want to thank my wife, who has been my friend, psychologist, nutritionist, and spiritual guide, my every thing during my time in Charlottesville. I want to thank my father mother and brother for their unconditional support. My discipline and self-drivability are qualities inherited from them. And I want to thank my mother in law, whose invaluable support came at a critical time. 


\section{Contents}

Contents

List of Figures . . . . . . . . . . . . . . . . . . . . . . . . . h

1 Introduction 1

1.1 Phonon Transport Across Interfaces . . . . . . . . . . . . . . . . . . . 3

2 Theoretical Background $\mathbf{8}$

2.1 Landauer viewpoint . . . . . . . . . . . . . . . . . . . . . . . . . 8

2.2 Landauer vs Boltzmann . . . . . . . . . . . . . . . . . . . . . . . . 11

2.3 Non-Equilibrium Greens Function: a brief overview . . . . . . . . . . . . . . . . . 13

2.3.1 From close to open systems . . . . . . . . . . . . . . . . . . . 13

2.3.2 Energy flow through the channel . . . . . . . . . . . . . . . . . . . . . . 14

3 Conductance Across One Boundary in 1D 18

3.1 Block Partition of 1D Chain and Contacts . . . . . . . . . . . . . . . . . . . . . 20

3.2 Transmission Using Blocks . . . . . . . . . . . . . . . . . . . . . . . . 22

3.2.1 Interface with Mass Junction . . . . . . . . . . . . . . . . . . . . . . . 23

3.2.2 Interface with Spring Junction . . . . . . . . . . . . . . . . . . . . . . . . 24

3.3 Optimally Coupled Interfaces . . . . . . . . . . . . . . . . . . . . . 25

3.3.1 Continuous vs. Discrete Limit . . . . . . . . . . . . . . . . . . . . . 26

3.3.2 Characteristics of OCI . . . . . . . . . . . . . . . . . . 28

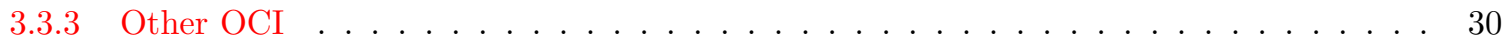

3.3.4 Extrapolation Example: Beyond Single Interfaces . . . . . . . . . . . . . . . . . . . 30

3.4 Summary of Contributions . . . . . . . . . . . . . . . . . . . . . . . . . . 32

4 Conductance Across Many Boundaries in 1D 33

4.1 Methodology . . . . . . . . . . . . . . . . . . . . . . . . 34

4.2 Single material, coherent.

Geometric Mean and anti-reflection coating. . . . . . . . . . . . . . . . . . . . . . . 35

4.3 Single material, Incoherent.

Still Geometric Mean. . . . . . . . . . . . . . . . . . . . . . . . . . . . . . . . . . . . 39

4.4 N Materials, Coherent.

Exponential. . . . . . . . . . . . . . . . . . . . . . . . . 40

4.5 N Materials, Incoherent.

Back to Exponential. . . . . . . . . . . . . . . . . . . . . . . . . . . . 43

4.6 Summary of Contributions . . . . . . . . . . . . . . . . . . . . . . . . . . 44

5 Conductance Across One Boundary in 3D 46

5.1 Methodology . . . . . . . . . . . . . . . . . . . . . . . . 47

5.2 Minimum vs. Conserving Modes . . . . . . . . . . . . . . . . . . . . . . 48

5.3 Gain vs. loss in Transmission . . . . . . . . . . . . . . . . . . . . . . . . . 54

5.4 Simulation details ... . . . . . . . . . . . . . . . . . . . 56

5.5 Polarization-Resolved Transmission . . . . . . . . . . . . . . . . . . 58 
5.6 Transmission for Mixed Interface . . . . . . . . . . . . . . . . . . . . . . . . 59

5.6.1 Equation Relating Amplitudes . . . . . . . . . . . . . . . . . . . . 59

5.6.2 Approximation of Amplitudes Equation . . . . . . . . . . . . . . . . . . . 61

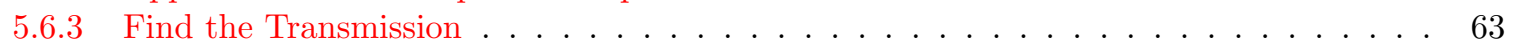

5.7 Summary of Contributions . . . . . . . . . . . . . . . . . . . . . . . 64

6 Conductance Across Many Boundaries in 3D 65

6.1 Thermal Conductance Varying Layer Mass and Length . . . . . . . . . . . . . . . . . . . . 65

6.2 Harmonic limit: Increasing Transmission vs. Decreasing Conserving Modes . . . . . . . . . . 68

6.3 Anharmonic limit: Decreasing Boundary Resistance vs. Increasing Intermediate Layer Resistance 71

6.4 Summary of Contributions . . . . . . . . . . . . . . . . . . . . 73

7 Conclusions and Future work 75

7.1 Conclusions ... . . . . . . . . . . . . . . . . . . . . . . 75

7.2 Future Work . . . . . . . . . . . . . . . . . . . . . . 75

8 Publications $\quad \mathbf{7 7}$

A NEGF Phonons

A.1 Problem and Approach: Big Picture . . . . . . . . . . . . . . . . . . . 78

A.2 Transport Formalism . . . . . . . . . . . . . . . . . . . . . . . . 79

A.2.1 System Equations: Preliminars . . . . . . . . . . . . . . . . . . . . . 79

A.2.2 Scattering Problem . . . . . . . . . . . . . . . . . . . . . 80

A.2.3 Classical Phonons . . . . . . . . . . . . . . . . . . . . . . . . . 82

A.2.4 Thermal Current Carried by Classical Phonons . . . . . . . . . . . . . . . . . . 83

A.2.5 Total Thermal Current . . . . . . . . . . . . . . . . . . . . . . . . 84

A.2.6 Transmission . . . . . . . . . . . . . . . . . . . . . . . . . 87

A.2.7 Local Density of States . . . . . . . . . . . . . . . . . . . . . . . . 88

A.2.8 Summery of Equations . . . . . . . . . . . . . . . . . . . . . . . 88

A.3 Transport in 1D Chain . . . . . . . . . . . . . . . . . . . . . . . . 89

A.3.1 Setting Up the Problem . . . . . . . . . . . . . . . . . . . . . 89

A.3.2 Analytical Example . . . . . . . . . . . . . . . . . . . . . . . 93

A.4 Transport in 3D Materials . . . . . . . . . . . . . . . . . . . . . . . . . 95

A.4.1 NEGF on an Infinite Crystalline Material . . . . . . . . . . . . . . . . . . . . . . 95

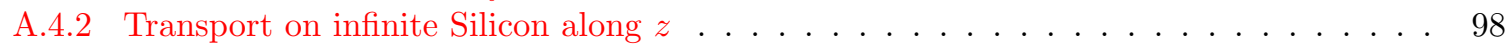

A.5 Orthogonalization of equation A.2 . . . . . . . . . . . . . . . . . . . 100

A.6 Summation in Terms of Green's Function for Orthonormal Basis . . . . . . . . . . . . . . . . 101

A.7 Summation in Terms of Green's Function for Non Orthonormal Basis . . . . . . . . . . . . . 102

A.8 A Identity . . . . . . . . . . . . . . . . . . . . . . . . . . 102

A.9 Computing Surface Green's Functions . . . . . . . . . . . . . . . . . . . . . . . 103

A.10 Dispersion Relation and Group velocity for 1D Analytical Example . . . . . . . . . . . . . . . 104

A.11 Si Force Constants from Harrison Potential . . . . . . . . . . . . . . . . . . . . . 105

A.11.1 Tensor Force Constants . . . . . . . . . . . . . . . . . . . . . . . . 105

A.11.2 Harrison Interatomic Potential . . . . . . . . . . . . . . . . . . . . . . . 106

A.11.3 Tensor Force Constants for Si . . . . . . . . . . . . . . . . . . . . . . . 107

A.11.4 Force Constants Relating Units Cells . . . . . . . . . . . . . . . . . . . . . . . 111

A.11.5 Dispersion Relation $\omega$ vs $k$. . . . . . . . . . . . . . . . . . . . . . . . . 112

A.11.6 Density of states . . . . . . . . . . . . . . . . . . . . . 114

B Anharmonicity 116

B.1 Quantum Harmonic Oscillator: 1D crystal . . . . . . . . . . . . . . . . . . 116

B.2 Green's Functions . . . . . . . . . . . . . . . . . . . . . 119

B.2.1 $G_{p}$ or $i D^{>} \ldots \ldots \ldots \ldots \ldots \ldots \ldots \ldots \ldots \ldots \ldots \ldots$

B.2.2 Retarded Green's Function . . . . . . . . . . . . . . . . . . . . . . . . . . 122

B.3 Average Harmonic Heat Current . . . . . . . . . . . . . . . . . . . . . . . . 123 
B.4 Conversion table . . . . . . . . . . . . . . . . . . . . . . . . . . . . . . . 124

B.4.1 Solution Procedure for Anharmonic NEGF . . . . . . . . . . . . . . . . . . . . . . 124

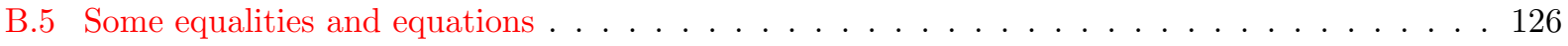

B.6 Delta function derivation . . . . . . . . . . . . . . . . . . . . 127

\section{Bibliography}




\section{List of Figures}

1.1 a): Equivalent thermal circuit of a chip [1] (TIM stands for thermal interface materials). b) and c) $25 \mathrm{~nm}$ gate length Silicon-on-Insulator (SOI) structure. b) Acoustic phonon temperature profile. c) Optical phonon temperature profile. . . . . . . . . . . . . . . . . . .

1.2 Problem statement: Given two materials, how do we couple them to get maximum thermal conductance across the junction? . . . . . . . . . . . . . . . . .

1.3 a) Measured thermal boundary resistance of several interfaces [2]. b Experimental data showing the dependence of thermal interfacial resistance with bonding strength [3]. c Experimental evidence of phonon ballistic transport [4]. $\mathrm{d}$ and e Experimental evidence of coherent transport.

1.4 Left) Thermal conductivity of Silicon using Landauer formalism [5]. Right) Thermal conductivity of GaAs using Landauer formalism coupled with interatomic force constants and scattering rates calculated from first principles $[$ Umpublished] $\ldots \ldots \ldots \ldots \ldots$

2.1 Breakdown of conductance Eq. 2.1. a) Factor $(\hbar \omega / 2 \pi) \partial N / \partial \mathbf{T}$, which filters the phonons that can contribute to conduction. At the Debye temperature of $\mathrm{Cu} \mathbf{T}_{D}^{C u}=315 \mathrm{~K}$ and $\mathrm{Si}$ $\mathbf{T}_{D}^{S i}=625 K$, the change in the factor over the allowed phonon range, denoted by the markers at the top and bottom axises, is around 10\%. b) Number of conducting channels per unit cell for a 1D chain, 2D square and 3D cubic lattices with $1^{\text {st }}$ nearest neighbor interaction. $\ldots .$.

3.1 a) $1 \mathrm{D}$ interface between dissimilar materials with an arbitrary atom in between. b) Optimal coupling between the contacts happens when we can describe the entire heterostructure only in terms of building blocks of individual materials. This decomposition makes the optimal atomic mass the arithmetic mean of its neighbors $m_{0}=m_{1} / 2+m_{2} / 2$. c) Interface with an arbitrary bond in between. d) Maximum thermal conductance occurs when the interfacial spring constant is the harmonic mean of its neighbors $1 / k_{0}=1 / 2 k_{1}+1 / 2 k_{2}$ (recall that a spring half as short is twice as strong), which follows again from a decomposition in terms of blocks of individual materials. . . . . . . . . . . . . . . . . .

3.2 a) $1 \mathrm{D}$ infinite chain of masses $m_{1}$, separated by $a$, joined by springs with force constant $k_{1} \cdot \mathbf{b}$ ) and c) Same chain separated into different blocks whose boundaries define different contacts. b) Partition into half-mass blocks with half masses across the boundary. c) Partition into half-spring blocks with half springs across the boundary. d) The half-spring blocks can be reinterpreted as half-mass blocks, provided the corresponding mass and spring constant are frequency dependent. The non-white background of the blocks represent this dependence. . .

3.3 Decomposition of interfaces into blocks for transmission calculations. An upper bound for transmission happens when the impurity atom $m_{i}$ or bond $1 / k_{i}$ are zero. a) Atom junction interface split into half-mass blocks. (b) Bond junction interface split into half-spring or (c) virtual half-mass blocks. In this case $u_{2 n}=A e^{i q_{1} n a}+B e^{i q_{1} n a} \ldots \ldots \ldots \ldots \ldots$

3.4 a) Optimally Coupled Interface and its representation as a single barrier for phonons, which is responsible for the scattering due to propagating waves changing medium. b) Non OCI and its representation as a step barrier plus an extra barrier caused by a deviation from the optimal case. This extra barrier decreases thermal conductance and can be associated with impurities, mixing or defects at the interface. . . . . . . . . . . . . . . . . 
3.5 Transmission of OCI visualized from the broadening of the contacts $\left(\Gamma_{1}\right.$ and $\left.\left.\left.\Gamma_{2}\right) . \mathbf{a}\right), \mathbf{b}\right)$ and $\mathbf{c}$ ) $\Gamma$ 's for half-mass contacts, half-spring contacts and half-spring, half-mass contacts respectively. The dotted lines represent $\Gamma_{2}$. d) Characteristic transmission function for all the cases before. At the particular frequency $\omega_{*}$, where $\Gamma$ s intersect each other, transmission becomes unity and the materials match. . . . . . . . . . . . . . . . . . .

3.6 1D interface between dissimilar materials with an arbitrary bond and atom in between. An OCI between half-spring and half-mass contacts arises when $m_{i}=0$ and $1 / k_{i}=0$. However, this OCI is an upper bound only for thermal conductance of systems in which $m_{0}$ vary arbitrarily and $1 / k_{i}=0$ or reciprocally. When $k_{0}$ and $m_{0}$ can vary together, interferences enter to the picture and the upper bound is lost. Interface with varying mass $m_{0}=m_{i}+\frac{m_{2}}{2}$ and spring $\frac{1}{k_{0}}=\frac{1}{k_{i}}+\frac{1}{2 k_{1}}$. The $1 \mathrm{D}$ chain modeled is obtained by combining the springs and masses when possible. . . . . . . . . . . . . . . . . . . . .

3.7 System consisting of two mediums sandwiching a third one characterized by the broadenings $\Gamma_{1}, \Gamma_{2}$ and $\Gamma_{0}$ respectively. Similar to the antireflection coating condition, transmission is unity when $L=\lambda / 4$ and $\Gamma_{0}=\sqrt{\Gamma_{1} \Gamma_{2}} \ldots \ldots \ldots \ldots \ldots \ldots$

3.8 Comparison of the transmission function predicted by Eq. 3.33 (dotted line) and its counterpart with the long wavelength constraint, i.e. Eq 3.33 replacing $\Gamma$ with $Z$ (solid line). The system consists only of half-mass blocks (Fig. 3.7) with $m_{1}=10^{-26} \mathrm{~kg}, \mathrm{~m}_{2}=10^{-24} \mathrm{~kg}$, $k_{1}=k_{2}=10 \mathrm{~N} / \mathrm{m}$ and $N_{b}=3 . m_{0}$ and $k_{0}$ were chosen to guarantee $T=1 @ \omega=4 \times 10^{12} \mathrm{rad} / \mathrm{s}$, i.e. $m_{0}=1.1779 \times 10^{-25} \mathrm{~kg}$ and $k_{0}=7.0338 \mathrm{~N} / \mathrm{m}$. The line with crosses represents the transmission of a system consisting only of half-spring blocks with the same parameters. . . .

4.1 Interfacial thermal conductance is maximized by inserting a graded junction with an exponentially varying "impedance" $\Gamma_{n}=\Gamma_{l} e^{\zeta n}$ (Eq. 4.18). However as the length between the contacts increases, the conductance gained by "impedance" matching is dominated by losses due to other scattering mechanisms. . . . . . . . . . . . . . . . . .

4.2 a) $1 \mathrm{D}$ crystal modeled as a chain of masses joined by springs. The masses at each contactjunction interface, balls with vertical lines, are chosen as the arithmetic mean of the neighboring masses to guarantee abrupt interfaces [6]. b) and c) The choice that maximizes $\langle T\rangle_{\omega}$ is an interplay between pushing the envelope up and fitting the right number of resonances inside the allowed frequency range. The figure shows an example where the largest envelope generated by the GM of contact broadenings (b) does not guarantee maximum $\langle T\rangle_{\omega}$ (c). It also shows the transmission for the abrupt interface between contacts $T_{l r}$ and the incoherent transmission $\langle T\rangle_{\varphi}$, which washes away interference (no wiggles). . . . . . . . . . . . . . .

4.3 (a) Choosing the impedance $Z_{1}=\sqrt{Z_{l} Z_{r}}$ does not guarantee high $\langle T\rangle_{\omega}$ because if the cut-off frequency lies below $\omega_{\max }$, tunneling cuts down transmission (shaded region). On the other hand, choosing the broadening $\Gamma_{1} \approx \sqrt{\Gamma_{l} \Gamma_{r}}$ (b) fixes a unique pair $m_{G M}, k_{G M}$ that favors the flow of both low and high frequency phonons. The figure shows a similar result for the incoherent transmission average $\left\langle\langle T\rangle_{\varphi}\right\rangle_{\omega}$. Note that $\Gamma$ for the maximum $\langle T\rangle_{\omega}\left(\Gamma_{\max }\right)$ intersects

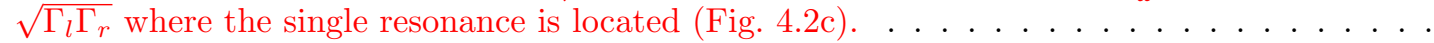

4.4 a) 1D crystal modeled as a chain of masses joined by springs. b) Transmission $T$ and its phase average $\langle T\rangle_{\text {}}$ for contacts coupled through an exponential variation of broadenings with a single junction $N=1$ and with $N=5$ junction materials. As we increase the number of materials transmission widens increasing $\langle T\rangle_{\omega}$. The spatial variation of acoustic impedance is shown in c) and of cut-off frequency in d) for $N=1$ and $N=5$. The crosses show the back of the envelope calculation given by Eq. 4.19. . . . . . . . . . . . . .

4.5 a) Using the exponential variation of broadenings, as the number of junction materials $N$ increases, $\langle T\rangle_{\omega}$ and $\left\langle\langle T\rangle_{\varphi}\right\rangle_{\omega}$ approach to one. b) As $\mathrm{N}$ increases the length between contacts increases and phonon flow is affected by scattering mechanism different from interfacial scattering. Therefore, there is a sweet spot for $N$ at which we stop increasing thermal conductance by adding junction materials. . . . . . . . . . . . . . . . . . . . .

5.1 a) Abrupt interface. b) Interface with random atomic mixing (mixed interface). c) Interface with an added homogeneous layer at the junction (uniform interface). . . . . . . . . . . 
5.2 Plot of the average $M T$ normalized by the results for an abrupt interface versus the average mass at the junction layer for the cases described at the beginning of the section. Adding mixing at an abrupt interface enhances the interfacial conductance in all systems simulated in this work, but the extent depends on crystal structure. Compared to uniform interfaces, however, mixing does not always yield an increase in conductance. . . . . . . . . . . . . .

5.3 a) For $\mathrm{SC}$, the conductance for the mixed interface is larger than the uniform interface because the loss in transmission, $T$, due to the extra scattering brought by the random atoms (area with vertical lines) is dominated by the gain in modes, $M$, coming from transmissions between phonon that do not conserve $k_{\perp}$ (area with horizontal lines). b) While the $M T_{\text {uni }}$ spectrum is limited by transmissions between phonon conserving $k_{\perp}$ (overlap region), the extra $M T_{\text {mix }}$ spectrum comes from transmissions between phonons that do not conserve $k_{\perp} \ldots \ldots \ldots$

5.4 a) For FCC, the conductance for the uniform interface is larger than the mixed interface because the loss in $T$ due to the extra scattering brought by the random atoms (area with vertical lines) dominates the gain in $M$ coming from transmissions between phonon that do not conserve perpendicular momentum (area with horizontal lines). b) $M_{c} \approx M_{\min }$ over most of the spectrum. Thus, mixing provides little advantage by allowing transmission between modes not conserving $k_{\perp} \ldots \ldots \ldots \ldots \ldots \ldots \ldots$

5.5 a) MT for DC crystal split in polarizations. The conductance of the mixed interface dominates mostly because mixing favors transmissions between TA-TA and TA-LA modes, where the ratio of $M_{\min } / M_{c}$ is larger. b) $M_{\min }$ and $M_{c}$ for the different polarization branches. . . . .

5.6 Available modes in the DC contacts for the (a) TA and (b) LA branches. The modes for SC crystal and the TA branches, where mixing enhances the most the conductance of an abrupt interface, present a central void that enhances the ratio $M_{\min } / M_{c} \ldots \ldots \ldots \ldots$

5.7 When the shift in the subbands dominate their distortion, the $k_{\perp}=0$ subband only covers a fraction of the whole band spectrum. This generates a void in the conserving modes, which is seen when mixing significantly enhances the conductance of an abrupt interface. The solid lines represent subbands for different $k_{\perp}$ and the shaded region is the projected dispersion on

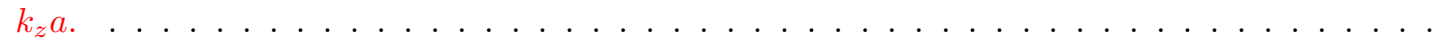

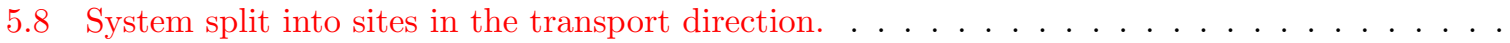

6.1 Schematic of a) an abrupt interface and b) a bridged interface with an added intermediate layer. 65

6.2 Conductance vs. junction atomic mass for different junction lengths. The IFCs come from the LJ interatomic potential. The conductance increases in the harmonic regime only $8 \%$, while the conductance increases when anharmonicity is present by about $33 \%$. This implies that anharmonic processes of phonons are the dominant factor for the enhancement of interfacial conductance, while impedance matching plays a minor role . . . . . . . . . . . . .

6.3 Conductance vs junction atomic mass for different junction lengths. The IFCs come from the SW interatomic potential. The conductance decreases in the harmonic regime when we add a junction material. This means that impedance matching is not useful to enhance conductance.

6.4 Extrapolation of the maximum relative conductance enhancement calculated by MD to $\boldsymbol{T}=0$. The extrapolation comes close to the NEGF result for very short layers but not for longer layers. This suggests the existence of an additional mechanism that is decreasing the conductance enhancement in the ballistic regime. The mechanism is a decrease in the number of conducting channels brought by an extra constraint on the modes that can conserve transverse momentum. 67

6.5 For single boundaries, as the ratio between the masses decreases, the interface thermal conductance increases as a result of an increase in both the number of conserving modes and

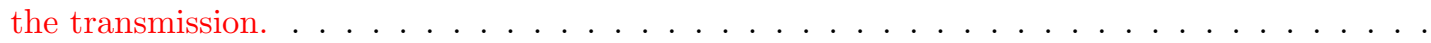

6.6 For an interface with an intermediate layer, the interface conductance results from an interplay between increasing the transmission due to decreasing the "mismatch" at each boundary and decreasing the number of conserving modes due to a new restriction coming from the

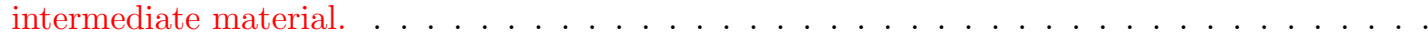


6.7 The figure shows the available modes for each material and how the adhesion material puts an extra constrain on the conserving modes. Thus hurting $M_{c}$ and decreasing the number of available modes. We plot the cases in which $G_{M_{c}}$ is minimum. (I should probably plot Mc for the abrupt interface also, that will allow a better comparison) $\ldots \ldots \ldots \ldots$

6.8 Conductance vs. junction atomic mass for two different $m_{r}$. We see a shift in the maximum conductance to the left. Note the non additivity for NEGF of resistances. I bet if I sum the

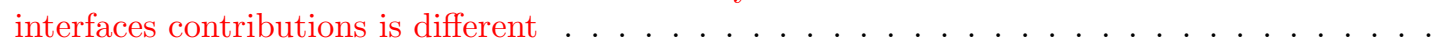

$6.9\langle M T\rangle_{A}$ for a single interface is a function of the ratio of the masses only. left this figure shows the $m_{J}$ that lead to minimum resistance calculated from $R_{T}=R_{1}+R_{2}$, the definition of $\mathrm{R}$ as a function of the cutoff frequency and AvgMT that depends on the mass ratio. This prediction based on single junction knowledge is contrasted vs the GM and the maxima from eyeballing from the MD results. Note that the $R_{T}$ are done summing the resistance from NEGF- the contacts resistance. The interpolation from MD have problems because is is not smooth and I get maxima at dirent places. Maybe the interpolation is not guaranteing the minimum increase

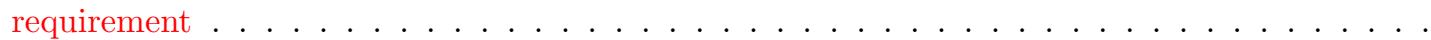

A.1 1D infinite chain of atoms split in three subsystems to apply NEGF formalism. Two contacts, characterized by atomic masses $m_{c 1}$ and $m_{c 2}$ and force constants $K^{c 1}$ and $K^{c 2}$ respectively, and a channel containing 3 atoms with masses $m_{1}, m_{2}$ and $m_{3}$ and 2 springs with force constants $K^{12}, K^{23}$. Contact $1(2)$ is coupled to the channel by a spring with force constant $K^{c 1}\left(K^{c 2}\right)$.

A.2 1D infinite chain with same mases $m=16.6 \times 10^{-27}[\mathrm{~kg}]$ and tensor force constants $K$. left) Transmission calculation right) Thermal conductance calculation. . . . . . . . . . . .

A.3 1D infinite chain of atoms separated a distance $a$ of mass $m_{0}$ related by springs with scalar force constant $K^{0}$. A single atom channel is chosen to work with scalars instead of matrices.

A.4 Left) System broken in slabs perpendicular to the transport direction (z). Right) Schematic representation of the unit cell $n_{p}$ in slab $z_{p} . m_{p}$ are the neighbors of $n_{p}$ in slab $z_{p}$, including itself, $m_{p+1}$ the neighbors of $n_{p}$ in slab $z_{p+1}$ and so on. $K^{m}$ is the block of the force constant matrix $K_{s y s}$ relating $\boldsymbol{u}_{n_{p}}$ with $\boldsymbol{u}_{m} \ldots \ldots \ldots \ldots \ldots \ldots$

A.5 Si unit cell $n_{0}$ with its nearest units cells. The different $x y$ squares represent different transport slabs. Left) Atom by atom picture. Each reddish atom joined by an orange bond with a bluish atom constitutes a unit cell. The lighter the color of the atom the larger its $z$ component. Middle) Schematic representation of the unit cells Right) Distances between the center unit cell $n_{0}$ and its neighbors $m \ldots \ldots \ldots \ldots \ldots$

A.6 up) Transmission $\Xi$ per unit area for Silicon calculated from NEGF down) Calculation by [7] 100

A.7 up) Thermal conductance per unit area (since $\Xi$ is per unit area) for Silicon calculated from NEGF down) Calculation by $[7] \ldots \ldots \ldots$. . . . . . . . . . . . . . . 100

A.8 $\omega$ vs $k$ dispersion of a 1D infinite chain of atoms separated by a distance $a$ of mass $m_{0}$ related by springs with scalar force constant $K^{0}$ (Figure A.3). The maximum allowed angular frequency

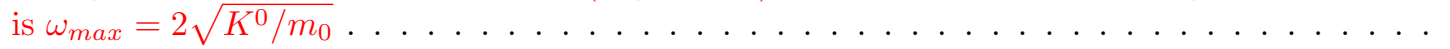

A.9 Numbering of the 16 nearest neighbors of site A and B. The reddish atoms occupy site A, the bluish atoms occupy site B. The lighter the color the larger the $z$ component of the atom. The orange bonds joined atoms in the same unit cell up Left) Neighbors of A up right) $x y$ view of the neighbors of A. Atom 4 and 6 are displaced from their equilibrium position (black circumference) as a visualization tool. The projected view only will show 4 since 6 is exactly under 4. Similarly with 8,10; 12,14 and 16,2 down Left) Neighbors of B down right $x y$ view of the neighbors of B. . . . . . . . . . . . . . . .

A.10 Unit cell $n_{0}$ with its 12 neighboring unit cells. Red bars represent coupling between unit cells. $\mathrm{d}$ and $\mathrm{u}$ stand for down and up. Table on the right shows the position vectors of each unit cell relative to the center unit cell. . . . . . . . . . . . . . . . . . .

A.11 Si dispersion relation Left using the force constants obtained with Harrison interatomic potential Right Experimental data by $[8] \ldots \ldots \ldots \ldots$

A.12 Density of States for Silicon using the force constants obtained with Harrison interatomic potential. Blue) calculation with NEGF. Red) calculation by binning. . . . . . . . . . . . 114 


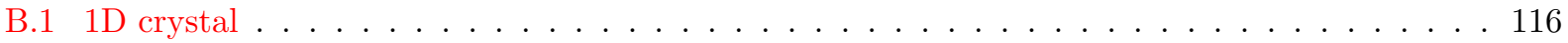




\section{Chapter 1}

\section{Introduction}

Atoms in solids are in constant motion oscillating around some equilibrium positions. The average amplitude of oscillation is proportional to the temperature. Thus, as the temperature of a system increases, the amplitude of the atomic motion also increases. This scatters more effectively electrons, increases the electronic resistance of the solid and degrades the quality of the material. In this way, an increase in temperature affects the performance, reliability and lifetime of almost any device, including MOSFETs, lasers, solar cells and batteries $[9,10,11]$.

One notable example is the CPUs clock frequency, which stabilized at around $2 \mathrm{GHz}$ when their dynamic power density became close to $100 \mathrm{~W} / \mathrm{cm}^{2}$ [12]. At that time, the concept of multi-core CPU came to rescue the increase in performance needed to continue the trend in economical growth. Nowadays, after some more generations of scaling keeping the rising performance trend, the passive power density due to leakage has also reached $100 \mathrm{~W} / \mathrm{cm}^{2}$ [12]. This increase in power density added to the increase in thermal resistance of individual transistors [10] have brought about another noticeable problem: Temperature inside the chip has risen to the point that the shell of our laptop can burn our laps. Unfortunately, that's not the only problem, temperature rise affects device reliability and lifetime [13].

As electrons move throughout devices and wires, they give away energy to the crystals causing a temperature increase inside a chip. The temperature $\boldsymbol{T}=R P$ depends on the amount of thermal energy generated per unit time within the system $P$; and on how easy this energy is dumped to the external world, which is determined by the thermal resistance $R$. Although in current devices, the dominant thermal resistance is on the package $R_{P A C K}$ (Fig. 1.1a), the resistance due to phonon flow in the neighborhood of devices $\left(R_{s c}\right)$ is already on the same order of magnitude and tends to increase as gate length decreases [1]. Thus, reducing $R_{s c}$ can cause a detectable reduction on the chip's temperature. For instance, a $50 \%$ reduction 
in $R_{s c}$ will decrease the peak temperature by approximately $5 \%$. A recent simulation on a Silicon-on-Insulator (SOI) device with $25 \mathrm{~nm}$ gate length showed more compelling evidence of the importance of $R_{s c}$ in nanodevices (Fig. 1.1b,c) [13]. For this particular device, a $10 \%$ reduction of $R_{s c}$ can reduce the temperature difference between source and drain by approximately $5 K$, effectively reducing the average temperature on the device.
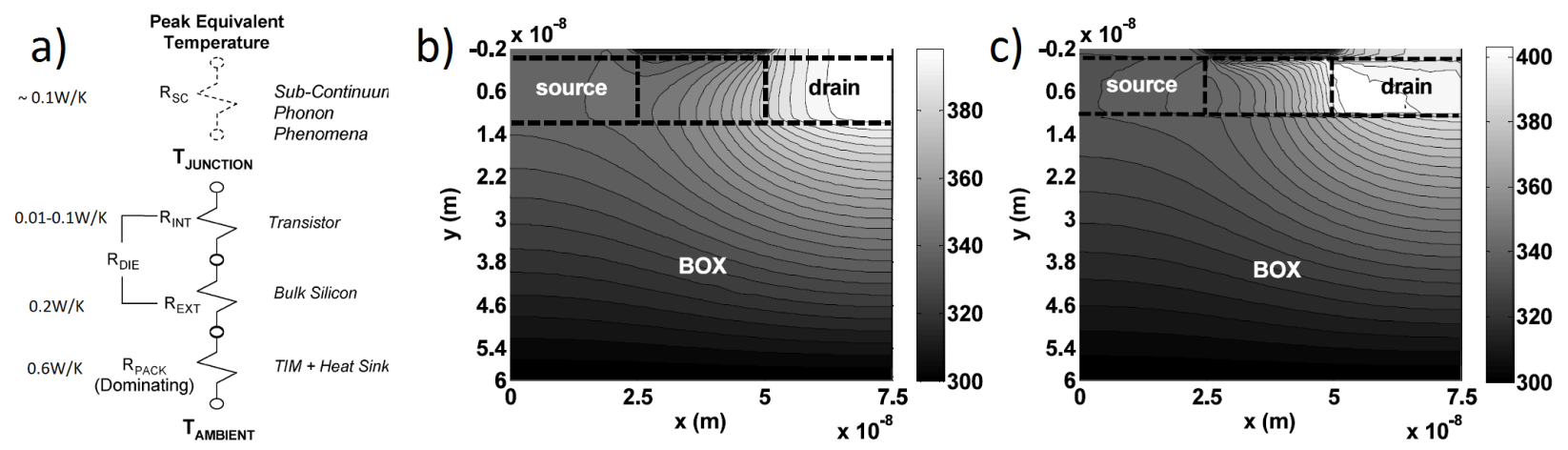

Figure 1.1: a): Equivalent thermal circuit of a chip [1] (TIM stands for thermal interface materials). b) and c) $25 \mathrm{~nm}$ gate length Silicon-on-Insulator (SOI) structure. b) Acoustic phonon temperature profile. c) Optical phonon temperature profile.

The role of interfaces on the total thermal resistance becomes increasingly important as the size of devices decreases. Thus, as we continue scaling down devices, interfaces will become the dominant factor of devices thermal resistance $\left(R_{s c}\right)$ [1]. For instance, thermal conductance measurement or various solid-solid interfaces spam from around 10 to $1000 \mathrm{M} \mathrm{W} /\left(\mathrm{m}^{2} \mathrm{~K}\right)$, which is equivalent to the conductance of a $\mathrm{SiO}_{2}$ sample with thickness between 100 to $1 \mathrm{~nm}$ [2]. In view of the gained importance of interfaces on the thermal resistance of devices at nanoscale, engineering interfaces to reduce their thermal resistance may be a way to ameliorate the high temperature problem of current and upcoming devices.

Understanding heat flow through interfaces is a hot research topic nowadays. A 2012 book chapter on thermal transport on nanostructured material stated: "In spite of 70 years of study of interfacial thermal transport, a detailed microscopic description of the Kapitza or interfacial resistance is still lacking" [14]. A 2013 News \& Views article on Nature Materials also stated in its title: "Understanding heat flow across interfaces remains an open question for thermal science" [15]. In order to address this scientific need of the community, which also could impact the technological problem of CPU heating, we post the following research problem: Given two materials, how do we couple them to get maximum thermal conductance across the junction? (Fig. 1.2).

The idea of introducing an intermediate layer to decrease interfacial resistance is motivated by concepts on electronic impedance matching and photonic antireflection coating, where an intermediate material is used to minimize signal or light reflection. Nevertheless, applying those concepts to the wavelike heat carriers, phonons, to minimize thermal resistance have three main challenges: 1) heat is carrier by a broadband 


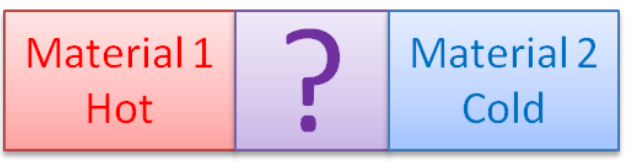

Figure 1.2: Problem statement: Given two materials, how do we couple them to get maximum thermal conductance across the junction?.

spectrum of interacting phonons instead of a single frequency non interacting electronic or photonic wave; 2) our understanding of phonon transport across interfaces is still incomplete, but it is essential to solve our problem; and 3) a set of principles that determine how to choose the properties of the interlayer material that minimize interface resistance does not exits.

To address the research question, we study thermal resistance across interfaces by varying the dimensionality of the system, the morphology of the interface, the crystal structure of the system, the length of the junction and the transport regime. We simulate the systems varying the properties of the intermediate layer in between the properties of the contacts. Then we explain the observed maximum conductance from physical principles related to the properties of the intermediate layer. For 1D chains, we simplify the system's interatomic force constants to scalars and we consider only first neighbor interactions between atoms. We study the question in both the coherent and incoherent elastic regimes. We study the effects of phonon-phonon interactions in NEGF by including the anharmonic cube perturbation to the harmonic force constants using self-consistent Born approximation. For 3D systems, we assume invariance of interatomic force constants, crystal structure and lattice constants along a particular system and only allow mass changes. In addition to the different intermediate material lengths studied, we consider the effect of interatomic mixing and different crystal structures. We perform our simulation in the coherent and incoherent elastic regime and compare those results with molecular dynamics simulations that include phonon-phonon interactions. The interatomic force constant where extracted from empirical potential as well as from first principles calculations.

\subsection{Phonon Transport Across Interfaces}

Pinpointing the mechanisms determining phonon flow through interfaces is a challenging task due to the many factors that affect the transport process $[2,16,9]$. On one hand, interfacial resistance depends on the phonon properties of the individual materials composing the interface, like group velocity, density of states or polarization. On the other hand, the resistance also depends on the atomic properties of the interface, like bonding strength, atomic mixing or defects density. From all those possibilities, figuring out the dominant 
mechanisms affecting thermal transport through interfaces requires a joint effort of experiments, simulations and theory.

Experimental works have already demonstrated the dependence of Thermal Boundary Conductance (TBC) on particular properties of the interface. For example, Hopkins et. al. showed a 30\% variation of TBC with interfacial mixing [17]. Losego et. al. showed 15\% variation of Au/SAM/Qz thermal resistance by changing the end chemistry of the molecule-Au bond (Fig. 1.3b) [3]. More details on the influence of roughness, disorder, dislocations and bonding on TBC can be found in a recent review on the topic [2]. Just to give you some prospective on the numbers, it is worth to notice that TBC of interfaces, where heat flow is dominated by phonons, extents over around 2 orders of magnitude. From Bi/Diamond with $T B C \approx 7$ $\mathrm{MW} / \mathrm{m}^{2} \mathrm{~K} @ \boldsymbol{T}=300 \mathrm{~K}$ to $\mathrm{TiN} / \mathrm{MgO}$ with $T B C \approx 700 \mathrm{MW} / \mathrm{m}^{2} \mathrm{~K} @ \boldsymbol{T}=300 \mathrm{~K}$ (Fig. 1.3a) [2]. Recently, two different conductivity measurements on superlattices have claimed the observation of coherent phonon transport (Fig. 1.3c, d and e) [4, 18]. Those controversial works support the importance of understanding ballistic, coherent and incoherent phonon flow in nanoscale devices.

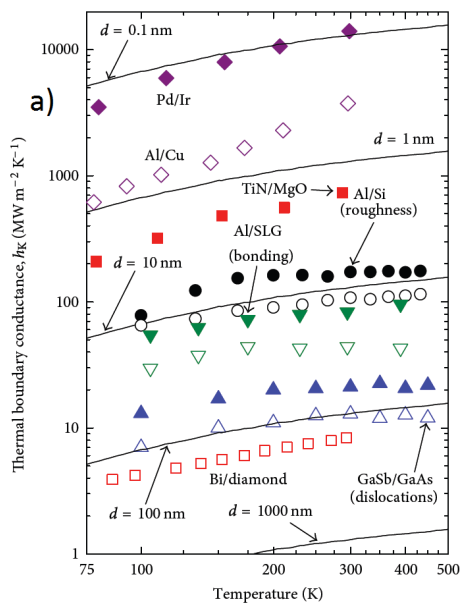

b)

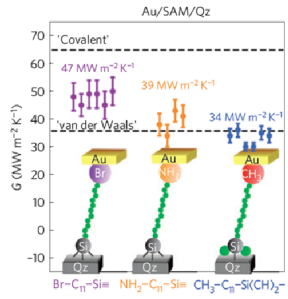

d)

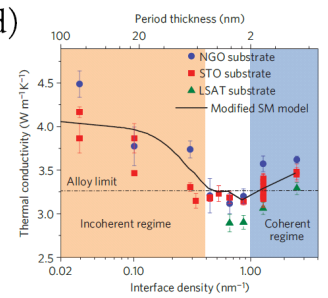

c)

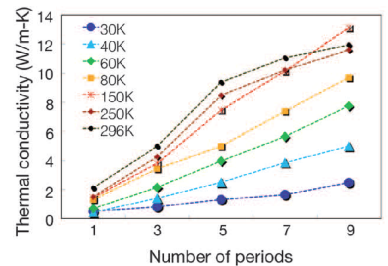

e)

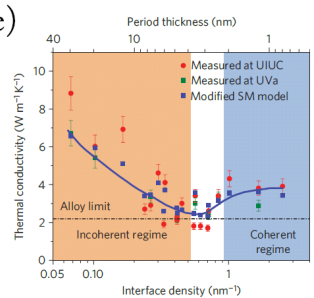

Figure 1.3: a) Measured thermal boundary resistance of several interfaces [2]. b Experimental data showing the dependence of thermal interfacial resistance with bonding strength [3]. c Experimental evidence of phonon ballistic transport [4]. d and e Experimental evidence of coherent transport.

From a theoretical point of view, describing phonon transport through interfaces is a demanding task. Heat is carried by a broad frequency spectrum of phonons with different properties. While, low frequency phonons feel average properties of crystals due to their long wavelength, high frequency phonons have shorter wavelength and mean free paths, are sensible to the atomic structure and present highly non linear dispersion relations. Additionally, phonons flow in different modes with different symmetries, so tensorial properties of materials and selection rules become important. At the interface, those phonons encounter defects, impurities and a handful of imperfections that mix them and scatter them. Moreover, when two interfaces are too close 
together $(\approx \mathrm{nm})$ wave interference entangles their individual contributions to the total thermal resistance in a non-additive way. Thus, finding a comprehensive theory is a daunting task.

Two semi-classical models to describe phonon transport through interfaces are widely used by the community because of their simplicity and low computational cost. The Acoustic Mismatch Model (AMM), which assumes coherent wave transport in continuum media and the Diffusive Mismatch Model (DMM), that assumes completely diffusive scattering at the interface [19]. Both models assume that thermal boundary resistance depends only on the properties of the individual materials composing the interface but not on the properties of the interface itself. This simple assumption is not enough to get quantitative agreement with experiments [14] and therefore, a lot of work is been devoted to include interfacial effects into the models. For instance, the effects of bonding strength [20], anharmonicity [21], more realistic density of states [22] or different types of diffusive scattering [23] have been studied but still, a complete picture is missing. An approach that has not been fully exploited by the community, but could produce some simple equations with quantitative prediction power, is the Landauer plus Non-Equilibrium Greens Formalism (NEGF) introduced by Datta [24]. This formalism has proven invaluable in bringing intuition to the mechanisms dictating electron flow at nanoscale and it is already starting to make its way into nanoscale phonon transport [5]. For instance, thermal conductivity of Silicon was calculated from the Landauer approach by including boundary scattering $\tau_{b}^{-1}=\frac{\langle v(\omega)\rangle}{F l}$, impurity scattering $\tau_{d}^{-1}=D \omega^{4}$ and unklapp scattering $\tau_{u}^{-1}=B \omega^{2} T e^{-C / T}$ into $\lambda=\frac{4}{3} v(\omega) \tau(\omega)$ [5]. We have also calculated the thermal conductivity of GaAs samples using Landauer formalism. The number of modes and mean free path are calculated from first principles interatomic force constants and scattering rates from Quantum Espresso [25] and ShengBTE [26].

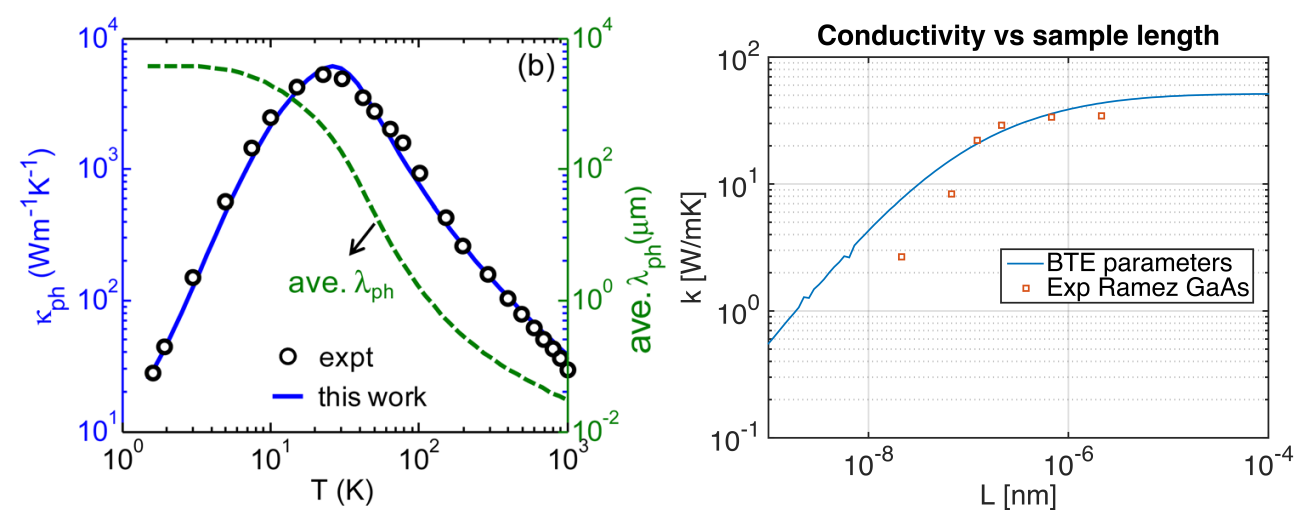

Figure 1.4: Left) Thermal conductivity of Silicon using Landauer formalism [5]. Right) Thermal conductivity of GaAs using Landauer formalism coupled with interatomic force constants and scattering rates calculated from first principles [Umpublished].

Several simulation techniques have been used to describe heat flow at nanoscale [14]. The methods based on solving Boltzmann Transport Equation (BTE) capture phonons statistics and can be coupled with first 
principle calculations, which make them suitable for predicting phonon flow at low temperatures in real materials. However, BTE works for phonon distributions close to equilibrium and assumes local thermal equilibrium, which automatically restricts the minimum size of the system to the order of the phonon mean free path, limiting the use of the method at nanoscale. Molecular Dynamics (MD) methods can capture anharmonicity and are suitable for systems at nanoscale. In fact, MD simulations are already doing a great job reproducing experimental trends and bringing physical insight. However, since this is a purely classical approach, it cannot capture quantum mechanical effects and it is not accurate at low temperature. Two commonly used MD methods are Non-equilibrium MD (NEMD) and Green-Kudo MD (GK-MD) which are based on linear response theory. The Wave Packet (WP) method is also a popular technique, which let a narrow wave packet of phonons propagate and interact with a particular nanostructure. The WP method comes useful to study properties of individual phonons. Another method being used is RTA-ED, which calculates the relaxation time of individual phonons from equilibrium MD and then uses the relaxation time approximation to calculate thermal conductivity. This method has computational advantages over the former explained MD methods. A more detailed explanation of these methods is developed in the excellent review by Chernatynskiy et. al. [14].

Recently, NEGF, a successful method for studying electron flow at nanoscale was adapted to phonon transport $[27,28,29,30,31]$. This method is a fully quantum mechanical treatment of phonon flow and can be coupled with first principle calculations. The approach can handle ballistic, diffusive and intermediate regimes. It doesn't rely on the knowledge of local temperature inside the nanostructure, which makes it the only method strictly valid for small nanostructures, since the notion of temperature becomes ill defined for domains of a few atoms. Aside from the computational load getting out of hands when the size of the system increases or when anharmonicity is included, this method offers the most complete description possible of heat flow and can be nicely complemented with MD simulations in case higher orders of anharmonicity are required.

In spite of all the advantages offered by NEGF, the method is not still widely used by the community, maybe because a link with the existing knowledge is missing. Most of the studies with NEGF are confined to ballistic transport, which limits their range of applicability to the effects of dimension mismatch or coherent scattering $[32,7,33,34,35,36,37]$ into thermal resistance. The force constants are in general obtained from inter-atomic potentials, which restrict the accuracy of NEGF to that of the inter-atomic potential $[32,7,33,34,35,36,37]$. Recent works have already used parameters from first principle calculations [38, 39, 40, 41, 42, 43, 44, 45], some of then obtained with Quantum Espresso (QE)[25]. The advantage of coupling Ab initio methods for the parameters with NEGF is the ability to make predictions without any a priori knowledge of the system. Very little work has been done on including the effects of anharmonicity 


\begin{tabular}{|c|c|c|c|c|c|}
\hline \multicolumn{7}{|c|}{ Studies of Interfacial Thermal Transport with NEGF } \\
\hline Ref. & Group & System & Anharmonic & Empirical & Ab initio \\
\hline$[43]$ & Cheng-MIT & Si/Ge (roughness) & No & Yes & Yes (Only for Si) \\
\hline$[4]$ & Cheng-MIT & GaAs/AlAs & No & Yes & No \\
\hline$[41]$ & Fisher-Purdue & TiC-GNR & No & No & Yes (Only for Bulk) \\
\hline$[50]$ & Fisher-Purdue & Polarization & No & Yes & No \\
\hline$[7]$ & Fisher-Purdue & Si/Ge & No & Harrison & No \\
\hline$[35]$ & Singapore & 3D-1D-3D (Buffer) & No & Yes & No \\
\hline$[48]$ & Singapore & Si-1D-Si & Yes & Lennard-Jones & No \\
\hline$[37]$ & Singapore & Si-1D-Si (Size, Doping) & No & Lennard-Jones & No \\
\hline$[33]$ & Wang-NUS & GNR/CBN & No & Tersoff & No \\
\hline$[34]$ & Yang-Colorado & Si/Ge (Size effects) & No & Tersoff & No \\
\hline$[36]$ & Yang-Colorado & Si/Ge (Reconstruction) & No & Tersoff & No \\
\hline$[49]$ & Luisier-Zurich & Si/Ge/Si nanowires & Yes & Yes & No \\
\hline
\end{tabular}

Table 1.1: Studies of interfacial phonon transport with NEGF. Acronyms: GNR-Graphene Nanoribons, CBN-Hexagonal Boron Nitride.

$[28,29,46,31,47,48,49]$ on NEGF and a calculation coupling QE with anharmonicity and NEGF has not been done yet. The inclusion of anharmonicity presents computational challenges and proper approximations could lead to ease the computational load. Approximations that tailor key physics in some limits while decreasing the computational load are needed.

Specific studies of interfacial phonon transport with NEGF are scarce (Tab. 1.1). Most of these works obtained the Inter-atomic Force Constants (IFC) using semi-empirical potentials [7, 48, 35, 50, 33, 37, 4, 34, $36,49]$, which restricts the accuracy of NEGF to that of the inter-atomic potential. Only two studies have used parameters from first principle calculations [41,43]. However, they only obtained ab initio IFC for the bulk materials composing the interface but not for the interface itself. Thus, the advantage brought by $a b$ initio calculations is limited by the approximations of IFC at the interface. More work is needed in finding good approximations and comparing them with complete ab initio simulations. Most of the studies artificially matched lattice structures at the interface and only 2 papers $[48,36]$ studied the effect of structural relaxation at the interface. Very little work has been done on including the effects of anharmonicity at the interface $[48,49]$ and a calculation coupling ab initio IFC, anharmonicity and NEGF has not been done yet. 


\section{Chapter 2}

\section{Theoretical Background}

\subsection{Landauer viewpoint}

Thermal conductance $G$ is defined as the ratio between heat flux $q$ and temperature drop $\Delta \mathbf{T}$. Within the Landauer theory this quantity can be expressed as [5]

$$
G=\frac{q}{\Delta \mathbf{T}}=\frac{I}{A \Delta \mathbf{T}}=\frac{1}{A} \int_{0}^{\infty} \frac{d \omega}{2 \pi} \hbar \omega \frac{\partial N}{\partial \mathbf{T}} M T
$$

where $I$ is the heat current, $A$ is the cross-sectional area, $\hbar \omega$ is the energy carried by a phonon, $N$ is the Bose-Einstein distribution

$$
N(\omega)=\frac{1}{1-\exp \left(\frac{\hbar \omega}{k_{B} T}\right)},
$$

with $k_{B}$ the Boltzmann constant; $M$ is the number of propagating modes, which we refer as "modes" throughout this document, and $T$ is the average transmission per mode. Note that $N, M$ and $T$ are all frequency dependent quantities. The factor $(\hbar \omega / 2 \pi) \partial N / \partial \mathbf{T}$ of Eq. 2.1, filters the phonons that can contribute to conduction (Fig. 2.1a). For low temperature, only low frequency, low energy phonons are allowed to contribute to transport. As the temperate increases, higher frequencies become available. At high temperatures (above the Debye temperature $\mathbf{T}_{D}$ ), the factor can be considered constant that equals $k_{B} / 2 \pi$. Similarly, in the classical limit, when $\hbar \rightarrow 0$ and $N \rightarrow k_{B} T /(\hbar \omega)$, Eq. 2.1 can be approximated as

$$
G=\frac{1}{A} \int_{0}^{\infty} \frac{d \omega}{2 \pi} k_{B} M T
$$



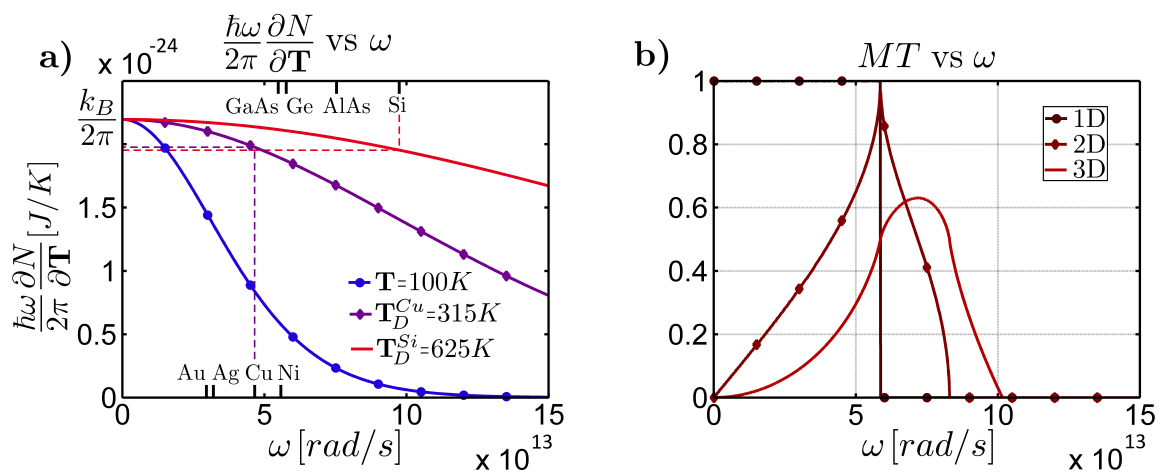

Figure 2.1: Breakdown of conductance Eq. 2.1. a) Factor $(\hbar \omega / 2 \pi) \partial N / \partial \mathbf{T}$, which filters the phonons that can contribute to conduction. At the Debye temperature of $\mathrm{Cu} \mathbf{T}_{D}^{C u}=315 K$ and Si $\mathbf{T}_{D}^{S i}=625 K$, the change in the factor over the allowed phonon range, denoted by the markers at the top and bottom axises, is around $10 \%$. b) Number of conducting channels per unit cell for a 1D chain, 2D square and 3D cubic lattices with $1^{\text {st }}$ nearest neighbor interaction.

For a given contact and frequency $\omega$, the propagating modes are the eigenvectors or plane wave solutions $\left(x_{n} \propto e^{i\left(k x_{n}-\omega t\right)}\right)$ of the equation of motion for the contact with eigenvalue $\omega^{2}$, with real wavevector $k$ and with group velocity in the transport direction. For homogeneous materials, $T=1$ and the factor $M T=M$. As shown in Fig. 2.1b, the number of conducting channels $M$ depends strongly on the dimension of the system.

The factor $M T$ on Eq. 2.1 captures the physics of phonon propagation through the device by adding the transmission probability for every pair of conducting channels on different contacts.

$$
M T=\sum_{k_{l}, k_{r}} T_{k_{l}, k_{r}}
$$

This quantity can be calculated from non-equilibrium Green's functions (NEGF) as $M T=\operatorname{Trace}\left\{\Gamma_{l} G_{r} \Gamma_{r} G_{r}^{\dagger}\right\}$, with $G_{r}$ the retarded Green's function and $\Gamma$ the broadening matrix for the left $(l)$ and right $(r)$ contacts, which is the anti-hermitian part of the self-energy and describes the interaction of the system with contact $[27,24,31]$

Eq. 2.1 with a phenomenological addition of non-coherent scattering can capture several transport regimes. We can recover the standard expression for thermal boundary conductance $h_{B D}[51]$ by noting that $M=\pi A D O S\left\langle v_{g}\right\rangle$, where $A$ is the cross sectional area, DOS is the density of states per unit volume and $\left\langle v_{g}\right\rangle$ is an average group velocity over all the phonons propagating in the positive transport direction with frequency $\omega$

$$
h_{B D}=\left[\frac{1}{2} \int_{0}^{\infty} d \omega \hbar \omega D O S\left\langle v_{g}\right\rangle T \frac{\partial N}{\partial \mathbf{T}}\right]
$$

To recover Fourier's law we assume $N_{s}$ identical scattering events that randomize the phase of the phonons 
and happen every $L_{s}$ distance. Then the phase average transmission $\langle T\rangle_{\varphi}$ satisfies the additive property $[52,53,24]$

$$
\frac{1-\langle T\rangle_{\varphi}}{\langle T\rangle_{\varphi}}=\sum_{j} \frac{1-T_{j}}{T_{j}}=N_{s} \frac{1-T_{s}}{T_{s}}=\frac{L}{L_{s}} \frac{1-T_{s}}{T_{s}},
$$

which can be expressed in the form

$$
\langle T\rangle_{\varphi}=\frac{\lambda}{\lambda+L} \quad \text { with } \quad \lambda=L_{s} \frac{T_{s}}{1-T_{s}},
$$

$L$ the device length and $\lambda$ the mean free path (mfp) for back-scattering [54]. Then we take the limit of long devices $L>>\lambda$, and use $\langle T\rangle_{\varphi} \approx \lambda / L$ instead of $T$ on Eq. 2.1. Just to make the point, consider a 3D isotropic material with a Debye like dispersion so the mfp for back-scattering $\lambda$ is $4 / 3$ of the $\operatorname{mfp} \Lambda$ and the average group velocity is half the slope of the dispersion $\left\langle v_{g}\right\rangle=v_{g} / 2$ [54]. Then the heat current is given by

$$
q A=\left[\int_{0}^{\infty} d \omega \frac{1}{3}\left(\hbar \omega D \frac{\partial N}{\partial \mathbf{T}}\right) v_{g} \Lambda\right] \frac{\Delta \mathbf{T}}{L} A .
$$

This equation is equivalent to Fourier's law

$$
q=-\kappa \nabla T=\kappa \frac{\Delta \boldsymbol{T}}{L}
$$

with the thermal conductivity $\kappa=1 / 3 C v \Lambda, C$ the heat capacity, $v$ the group velocity and $\Lambda$ the mean free path.

From Eq. 2.1, we can also get the spectral thermal conductivity as

$$
\kappa(\omega)=G(\omega) L=\frac{\hbar \omega}{2 \pi} \frac{\partial N}{\partial \boldsymbol{T}} \frac{L}{A} M T,
$$

using the definition of number of modes $M[5,54]$

$$
M=\frac{\pi}{L} \sum_{k, s} \delta\left(\omega-\omega_{k, s}\right)\left|v_{z}\right|
$$

and in the limit of long devices, where the transmission is given by $T=\lambda_{k, s} / L$ (Note that $\omega_{k, s}$ is the frequency of the $k, s$ normal mode, $k$ labels the wavevector, $s$ labels the polarization and $v_{z}$ is the group velocity of the mode labeled by $k, s$ in the direction of transport $z$ ), the spectral conductivity is given by

$$
\kappa(\omega)=\frac{\hbar \omega}{2 \pi} \frac{\partial N}{\partial \boldsymbol{T}} \frac{L}{A} \frac{\pi}{L} \sum_{k, s} \delta\left(\omega-\omega_{k, s}\right)\left|v_{z}\right| \frac{\lambda_{k, s}}{L}
$$




$$
\kappa(\omega)=\frac{1}{V} \hbar \omega \frac{\partial N}{\partial \boldsymbol{T}} \sum_{k} \delta\left(\omega-\omega_{k, s}\right) \frac{\left|v_{z}\right|}{2} \lambda_{k, s}
$$

\subsection{Landauer vs Boltzmann}

From Boltzmann transport equation within the relaxation time approximation, the heat flux in the transport direction $z$ on a homogeneous material is given by (This section follows closely reference $[54,5]$ )

$$
q_{z}(\omega)=-\frac{1}{V} \hbar \omega \frac{\partial N}{\partial \boldsymbol{T}} \sum_{k, s} \delta\left(\omega-\omega_{k, s}\right) v_{z}^{2} \tau_{k, s} \frac{\partial \boldsymbol{T}}{\partial z}
$$

where $V$ is the volume, $k$ labels the wavevector, $s$ labels the polarization, $v_{z}$ is the group velocity component in the transport direction and $\tau_{k, s}$ is the relaxation time of the mode labeled by $k, s$. Defining

$$
q_{z}=-\kappa \frac{\partial \boldsymbol{T}}{\partial z}
$$

the spectral thermal conductivity $\kappa$ is given by

$$
\kappa=\frac{1}{V} \hbar \omega \frac{\partial N}{\partial \boldsymbol{T}} \sum_{k, s} \delta\left(\omega-\omega_{k, s}\right) v_{z}^{2} \tau_{k, s}
$$

Comparing Eq. 2.11 and 2.13, we can define the mean free path to be used in Landaur theory as

$$
\lambda_{k, s}=2\left|v_{z}\right| \tau_{k, s}
$$

Because of the two in front, the $\lambda$ used in Landauer is the mean free path for back scattering. Now that we have a clear definition for the mfp for back scattering to be used in Landauer theory $\lambda_{k, s}$ in terms of the scattering time, we can use the scattering time calculated from the solution of Boltzmann transport equation in our Landauer theory.

To find the size effects on a sample, we can go back to Landauer's definition of spectral conductivity and replace $T=\lambda / L$ by $T=\lambda /(\lambda+L)$ in Eq. 2.10. In this way we calculate the thermal conductivity of a finite length GaAs sample shown in Fig. 1.4b. The mentioned replacement is equivalent to the definition of an effective scattering time using Matthiessen's rule between the bulk and boundary scattering times. Indeed, 
replacing $T=\lambda /(\lambda+L)$ and Eq. 2.14 in Eq. 2.10, we have

$$
\begin{aligned}
\kappa(\omega) & =\frac{\hbar \omega}{2 \pi} \frac{\partial N}{\partial \boldsymbol{T}} \frac{L}{A} \frac{\pi}{L} \sum_{k, s} \delta\left(\omega-\omega_{k, s}\right)\left|v_{z}\right| \frac{\lambda_{k, s}}{\lambda_{k, s}+L} \\
& =\frac{1}{V} \hbar \omega \frac{\partial N}{\partial \boldsymbol{T}} \sum_{k, s} \delta\left(\omega-\omega_{k, s}\right)\left|v_{z}\right| \frac{L}{2} \frac{2\left|v_{z}\right| \tau_{k, s}}{2\left|v_{z}\right| \tau_{k, s}+L} \\
& =\frac{1}{V} \hbar \omega \frac{\partial N}{\partial \boldsymbol{T}} \sum_{k, s} \delta\left(\omega-\omega_{k, s}\right) v_{z}^{2} \frac{1}{\frac{1}{\tau_{k, s}}+\frac{2\left|v_{z}\right|}{L}} \\
& =\frac{1}{V} \hbar \omega \frac{\partial N}{\partial \boldsymbol{T}} \sum_{k, s} \delta\left(\omega-\omega_{k, s}\right) v_{z}^{2} \tau_{e f f},
\end{aligned}
$$

where the effective scattering time is defined by

$$
\frac{1}{\tau_{e f f}}=\frac{1}{\tau_{k, s}}+\frac{1}{\tau_{B}}
$$

and $\tau_{B}$ is the boundary scattering

$$
\tau_{B}=\frac{L}{2\left|v_{z}\right|} .
$$

Many times, our knowledge of the scattering times is limited, so we only have access to scattering times dependent on frequency, loosing the resolution of particular wavevectors and polarizations. For instance, the commonly used expressions for anharmonic and impurity scattering [55] work this way:

$$
\frac{1}{\tau_{a}}=\frac{1}{B T \omega^{2} e^{-C / \boldsymbol{T}}} \quad \frac{1}{\tau_{i}}=\frac{1}{A \omega^{4}} .
$$

To define a mean free path for this situation, let us start from the spectral conductivity derived from Boltzmann transport equation (Eq. 2.13) and rewrite it in terms of quantities averaged over the wavevectors and polarizations

$$
\begin{gathered}
\left\langle v_{z}^{2} \tau\right\rangle=\frac{\sum_{k, s} \delta\left(\omega-\omega_{k, s}\right) v_{z}^{2} \tau}{\sum_{k, s} \delta\left(\omega-\omega_{k, s}\right)}, \\
D=\sum_{k, s} \delta\left(\omega-\omega_{k, s}\right),
\end{gathered}
$$

and

$$
\left\langle\left|v_{z}\right|\right\rangle=\frac{\sum_{k, s} \delta\left(\omega-\omega_{k, s}\right)\left|v_{z}\right|}{\sum_{k, s} \delta\left(\omega-\omega_{k, s}\right)},
$$


so the spectral conductivity is given by

$$
\kappa=\frac{1}{V} \hbar \omega \frac{\partial N}{\partial \boldsymbol{T}} D\left\langle\left|v_{z}\right|\right\rangle \frac{\left\langle v_{z}^{2} \tau\right\rangle}{\left\langle\left|v_{z}\right|\right\rangle}
$$

Note that $D$ is the density of states, which is different from the density of states per unit volume $D O S$ defined before. Now we rewrite the number of modes from Eq. 2.9

$$
M=\frac{\pi}{L} \sum_{k, s} \delta\left(\omega-\omega_{k, s}\right)\left|v_{z}\right|=\frac{\pi}{L}\left\langle\left|v_{z}\right|\right\rangle D
$$

and replace it on Eq. 2.25 so that the spectral conductivity becomes

$$
\kappa=\frac{\hbar \omega}{2 \pi} \frac{\partial N}{\partial \boldsymbol{T}} \frac{M}{A} \frac{2\left\langle v_{z}^{2} \tau\right\rangle}{\left\langle\left|v_{z}\right|\right\rangle} .
$$

We can compare with Landauer spectral conductance from Eq. 2.8 in the diffusive limit where $T=\lambda_{\omega} / L$

$$
\kappa(\omega)=\frac{\hbar \omega}{2 \pi} \frac{\partial N}{\partial \boldsymbol{T}} \frac{M}{A} \lambda_{\omega}
$$

Then, the mean free path for back scattering to use in Landauer theory is given by

$$
\lambda_{\omega}=\frac{2\left\langle v_{z}^{2} \tau\right\rangle}{\left\langle\left|v_{z}\right|\right\rangle}
$$

\subsection{Non-Equilibrium Greens Function: a brief overview}

In the following section we describe the basic equations of the non-equilibrium Green's function method. A more detail description of the method and a description of how to include anharmonic phonon-phonon interactions is given in the Appendix.

\subsubsection{From close to open systems}

Consider a huge contact in thermal equilibrium, whose equation of motion is given by

$$
\left[\omega^{2} M_{r_{i}}-F_{r_{i}}+i \eta\right] \boldsymbol{x}_{r_{i}}=S^{\prime}
$$

where $M_{r_{i}}$ is a diagonal matrix containing the masses of each atom, $F_{r_{i}}$ is the matrix of force constants relating neighboring atoms, $\boldsymbol{x}_{r_{i}}$ is a vector containing the displacements from equilibrium of each atom, $\eta$ is 
an infinitesimal positive number and $S^{\prime}$ is chosen to cancel $i \eta \boldsymbol{x}_{r_{i}}$. Also consider an isolated channel described by

$$
\left[\omega^{2} M-F\right] \boldsymbol{x}=0,
$$

with $M, F$ and $\boldsymbol{x}$ the mass matrix, force constant matrix and displacement vector of the atoms in the channel. When we couple those two systems together by linking their constituting atoms with force constants described by the matrix $\tau_{r_{i}}$, the energy stored on the atoms from the contact, which is at thermal equilibrium, will try to flow to the channel creating a motion on the atoms on the channel $\boldsymbol{x}$ and distorting the existing motion on the contact atoms by $\boldsymbol{y}_{r_{i}}$. Then the coupled system can be described by the following equation of motion:

$$
\left[\begin{array}{cc}
\omega^{2} M-F & -\tau_{r_{i}} \\
-\tau_{r_{i}}^{\dagger} & \omega^{2} M_{r_{i}}-F_{r_{i}}+i \eta
\end{array}\right]\left[\begin{array}{c}
\boldsymbol{x} \\
\boldsymbol{x}_{r_{i}}+\boldsymbol{y}_{r_{i}}
\end{array}\right]=\left[\begin{array}{c}
0 \\
S^{\prime}
\end{array}\right]
$$

From this matrix equation we can solve for $\boldsymbol{x}$ and obtain the displacements the we are interested in term of the somehow "known" displacements of the contact

$$
\begin{aligned}
\boldsymbol{x} & =G_{r} S \\
G_{r} & =\left[\omega^{2} M-K-\Sigma\right]^{-1} \\
\Sigma & =\sum_{r_{i}} \Sigma_{r_{i}}=\sum_{r_{i}} \tau_{r_{i}} G_{r_{i}} \tau_{r_{i}}^{\dagger} \\
G_{r_{i}} & =\left[\omega^{2} M_{r_{i}}-K_{r_{i}}+i \eta\right]^{-1} \\
S & =\sum_{r_{i}} S_{r_{i}}=\sum_{r_{i}} \tau_{r_{i}} \boldsymbol{x}_{r_{i}} \\
\boldsymbol{y}_{r_{i}} & =G_{r_{i}} \tau_{r_{i}}^{\dagger} \boldsymbol{x} \\
A_{r_{i}} & =i\left(G_{r_{i}}-G_{r_{i}}^{\dagger}\right) \\
A & =i\left(G_{r}-G_{r}^{\dagger}\right) \\
\Gamma_{r_{i}} & =i\left(\Sigma_{r_{i}}-\Sigma_{r_{i}}^{\dagger}\right)=\tau_{r_{i}} A_{r_{i}} \tau_{r_{i}}^{\dagger}
\end{aligned}
$$

In this way we turn a huge close system problem into an small open system problem.

\subsubsection{Energy flow through the channel}

The energy associated with the channel is given by

$$
H=\frac{1}{2} \dot{\boldsymbol{x}}^{\dagger} M \dot{\boldsymbol{x}}+\frac{1}{2} \boldsymbol{x}^{\dagger} F \boldsymbol{x}+\frac{1}{4}\left(\boldsymbol{x}_{r_{i}}+\boldsymbol{y}_{r_{i}}\right)^{\dagger} \tau_{r_{i}}^{\dagger} \boldsymbol{x}+\frac{1}{4} \boldsymbol{x}^{\dagger} \tau_{r_{i}}\left(\boldsymbol{x}_{r_{i}}+\boldsymbol{y}_{r_{i}}\right)
$$


We want to find the average change of energy with respect to time $d H / d t$, which is the energy current. The average will be critical, because we only know average properties of the contacts. To find that change we will use the equation of motion in time to get rid of the second derivatives in time, since

$$
\left[\begin{array}{cc}
M & 0 \\
0 & M_{r_{i}}
\end{array}\right]\left[\begin{array}{c}
\ddot{\boldsymbol{x}} \\
\ddot{\boldsymbol{x}}_{r_{i}}+\ddot{\boldsymbol{y}}_{r_{i}}
\end{array}\right]=-\left[\begin{array}{cc}
F & \tau_{r_{i}} \\
\tau_{r_{i}}^{\dagger} & F_{r_{i}}
\end{array}\right]\left[\begin{array}{c}
\boldsymbol{x} \\
\boldsymbol{x}_{r_{i}}+\boldsymbol{y}_{r_{i}}
\end{array}\right],
$$

which leads to

$$
\begin{gathered}
M \ddot{\boldsymbol{x}}=-F \boldsymbol{x}-\tau_{r_{i}}\left(\boldsymbol{x}_{r_{i}}+\boldsymbol{y}_{r_{i}}\right) \\
\ddot{\boldsymbol{x}}^{\dagger} M=-\boldsymbol{x}^{\dagger} F-\left(\boldsymbol{x}_{r_{i}}^{\dagger}+\boldsymbol{y}_{r_{i}}^{\dagger}\right) \tau_{r_{i}}^{\dagger} .
\end{gathered}
$$

Then, the change of energy with respect to time is given by

$$
\begin{aligned}
\frac{d}{d t} H= & \frac{d}{d t} \operatorname{Tr}\{H\}=\operatorname{Tr}\left\{\frac{d}{d t} H\right\} \\
= & \operatorname{Tr}\left\{\frac{1}{2} \ddot{\boldsymbol{x}}^{\dagger} M \dot{\boldsymbol{x}}+\frac{1}{2} \dot{\boldsymbol{x}}^{\dagger} M \ddot{\boldsymbol{x}}+\frac{1}{2} \dot{\boldsymbol{x}}^{\dagger} F \boldsymbol{x}+\frac{1}{2} \boldsymbol{x}^{\dagger} F \dot{\boldsymbol{x}}+\frac{1}{4}\left(\dot{\boldsymbol{x}}_{r_{i}}+\dot{\boldsymbol{y}}_{r_{i}}\right)^{\dagger} \tau_{r_{i}}^{\dagger} \boldsymbol{x}\right. \\
& \left.+\frac{1}{4}\left(\boldsymbol{x}_{r_{i}}+\boldsymbol{y}_{r_{i}}\right)^{\dagger} \tau_{r_{i}}^{\dagger} \dot{\boldsymbol{x}}+\frac{1}{4} \dot{\boldsymbol{x}}^{\dagger} \tau_{r_{i}}\left(\boldsymbol{x}_{r_{i}}+\boldsymbol{y}_{r_{i}}\right)+\frac{1}{4} \boldsymbol{x}^{\dagger} \tau_{r_{i}}\left(\dot{\boldsymbol{x}}_{r_{i}}+\dot{\boldsymbol{y}}_{r_{i}}\right)\right\} \\
= & \operatorname{Tr}\left\{\frac{1}{4}\left(\dot{\boldsymbol{x}}_{r_{i}}+\dot{\boldsymbol{y}}_{r_{i}}\right)^{\dagger} \tau_{r_{i}}^{\dagger} \boldsymbol{x}-\frac{1}{4}\left(\boldsymbol{x}_{r_{i}}+\boldsymbol{y}_{r_{i}}\right)^{\dagger} \tau_{r_{i}}^{\dagger} \dot{\boldsymbol{x}}-\frac{1}{4} \dot{\boldsymbol{x}}^{\dagger} \tau_{r_{i}}\left(\boldsymbol{x}_{r_{i}}+\boldsymbol{y}_{r_{i}}\right)+\frac{1}{4} \boldsymbol{x}^{\dagger} \tau_{r_{i}}\left(\dot{\boldsymbol{x}}_{r_{i}}+\dot{\boldsymbol{y}}_{r_{i}}\right)\right\}
\end{aligned}
$$

At this point, we replace the time derivative on the right factors by $-i \omega$ times the factor, for example we replace $\dot{\boldsymbol{x}}_{r_{i}}^{\dagger} \tau_{r_{i}}^{\dagger} \boldsymbol{x}$ with $-i \omega \boldsymbol{x}_{r_{i}}^{\dagger} \tau_{r_{i}}^{\dagger} \boldsymbol{x}$. We also replace the time derivative on the left factors by $i \omega$ times the factor, for example we replace $\boldsymbol{x}_{r_{i}}^{\dagger} \tau_{r_{i}}^{\dagger} \dot{\boldsymbol{x}}$ by $i \omega \boldsymbol{x}_{r_{i}}^{\dagger} \tau_{r_{i}}^{\dagger} \boldsymbol{x}$. The formal derivation of the replacement requires using the second quantized description of the problem and the thermal average [31, 28]. Here, we use an informal simplification that simplifies the treatment. Then

$$
\frac{d}{d t} H=\frac{1}{2} \operatorname{Tr}\left\{-i \omega\left(\boldsymbol{x}_{r_{i}}+\boldsymbol{y}_{r_{i}}\right)^{\dagger} \tau_{r_{i}}^{\dagger} \boldsymbol{x}+i \omega \boldsymbol{x}^{\dagger} \tau_{r_{i}}\left(\boldsymbol{x}_{r_{i}}+\boldsymbol{y}_{r_{i}}\right)\right\}
$$


Replacing $\boldsymbol{x}=G_{r} S, S_{r_{i}}=\tau_{r_{i}} \boldsymbol{x}_{r_{i}}$ and $\boldsymbol{y}_{r_{i}}=G_{r_{i}} \tau_{r_{i}}^{\dagger} \boldsymbol{x}$, and using $A=i\left(G_{r}-G_{r}^{\dagger}\right)$ and $\Gamma_{r_{i}}=i\left(\Sigma_{r_{i}}-\Sigma_{r_{i}}^{\dagger}\right)$, we have

$$
\begin{aligned}
\frac{d}{d t} H & =\frac{1}{2} \operatorname{Tr}\left\{-i \omega S_{r_{i}}^{\dagger} G_{r} S_{r_{i}}-i \omega \boldsymbol{x}^{\dagger} \tau G_{r_{i}}^{\dagger} \tau_{r_{i}}^{\dagger} \boldsymbol{x}+i \omega S_{r_{i}}^{\dagger} G_{r}^{\dagger} S_{r_{i}}+i \omega \boldsymbol{x}^{\dagger} \tau_{r_{i}} G_{r_{i}} \tau_{r_{i}}^{\dagger} \boldsymbol{x}\right\} \\
\frac{d}{d t} H & =\frac{1}{2} \operatorname{Tr}\left\{-\omega S_{r_{i}}^{\dagger} i\left(G_{r}-G_{r}^{\dagger}\right) S_{r_{i}}+\omega \boldsymbol{x}^{\dagger} i\left(\Sigma_{r_{i}}-\Sigma_{r_{i}}^{\dagger}\right) \boldsymbol{x}\right\} \\
\frac{d}{d t} H & =\frac{1}{2} \operatorname{Tr}\left\{-\omega S_{r_{i}}^{\dagger} A S_{r_{i}}+\omega \boldsymbol{x}^{\dagger} \Gamma_{r_{i}} \boldsymbol{x}\right\} .
\end{aligned}
$$

Reorganizing the equation, taking the thermal average and defining

$$
\Sigma_{r_{i}}^{i n}=\frac{1}{\hbar}\left\langle S_{r_{i}} S_{r_{i}}^{\dagger}\right\rangle \quad G^{n}=\frac{1}{\hbar}\left\langle\boldsymbol{x} \boldsymbol{x}^{\dagger}\right\rangle
$$

we have

$$
\begin{aligned}
\frac{d}{d t}\langle H\rangle & =-\frac{1}{2} \hbar \omega \operatorname{Tr}\left\{\left\langle S_{r_{i}} S_{r_{i}}^{\dagger}\right\rangle A-\Gamma_{r_{i}}\left\langle\boldsymbol{x} \boldsymbol{x}^{\dagger}\right\rangle\right\} \\
\frac{d}{d t}\langle H\rangle & =-\frac{1}{2} \hbar \omega \operatorname{Tr}\left\{\Sigma_{r_{i}}^{i n} A-\Gamma_{r_{i}} G^{n}\right\}
\end{aligned}
$$

To get the outgoing energy current (from the channel to the contact, i.e. the negative of what we just find), we make the inverse Fourier transform of the current. Note that the integral is taken over a frequency range varying from $-\infty$ to $\infty$.

$$
\begin{aligned}
I_{r_{i}}(t) & =\int_{-\infty}^{\infty} \frac{d \omega}{2 \pi} \frac{1}{2} \hbar \omega \operatorname{Tr}\left\{\Sigma_{r_{i}}^{i n} A-\Gamma_{r_{i}} G^{n}\right\} e^{-i \omega t} \\
I_{r_{i}}(0) & =\int_{-\infty}^{\infty} \frac{d \omega}{2 \pi} \frac{1}{2} \hbar \omega \operatorname{Tr}\left\{\Sigma_{r_{i}}^{i n} A-\Gamma_{r_{i}} G^{n}\right\} \\
I_{r_{i}} & =\int_{0}^{\infty} \frac{d \omega}{2 \pi} \hbar \omega \operatorname{Tr}\left\{\sum_{r_{i}}^{i n} A-\Gamma_{r_{i}} G^{n}\right\}
\end{aligned}
$$

For a system connected to a left $(l)$ and right $(r)$ contacts in the harmonic regime, we can get the commonly used formula for transmission by noting that $\Sigma_{l}^{i n}=\Gamma_{l} N_{l}, A=G_{r}\left(\Gamma_{l}+\Gamma_{r}\right) G_{r}^{\dagger}$ and $G^{n}=$ $G_{r} \Sigma^{i n} G_{r}^{\dagger}=N_{l} G_{r} \Gamma_{l} G_{r}^{\dagger}+N_{r} G_{r} \Gamma_{r} G_{r}^{\dagger}$. Then we have

$$
\begin{aligned}
& \Sigma_{l}^{i n} A-G^{n} \Gamma_{l}=\Gamma_{l} G_{r} \Gamma_{l} G_{r}^{\dagger} N_{l}+\Gamma_{l} G_{r} \Gamma_{r} G_{r}^{\dagger} N_{l}-\Gamma_{l} G_{r} \Gamma_{l} G_{r}^{\dagger} N_{l}-\Gamma_{l} G_{r} \Gamma_{r} G_{r}^{\dagger} N_{r} \\
& \Sigma_{l}^{i n} A-G^{n} \Gamma_{l}=\Gamma_{l} G_{r} \Gamma_{r} G_{r}^{\dagger}\left(N_{l}-N_{r}\right) .
\end{aligned}
$$


Then, the current becomes

$$
I_{r_{i}}=\int_{0}^{\infty} \frac{d \omega}{2 \pi} \hbar \omega \operatorname{Tr}\left\{\Gamma_{l} G_{r} \Gamma_{r} G_{r}^{\dagger}\right\}\left(N_{l}-N_{r}\right),
$$

which compared to Eq. 2.1 tell us that $M T=\operatorname{Tr}\left\{\Gamma_{l} G_{r} \Gamma_{r} G_{r}^{\dagger}\right\}$

A brief description of the anharmonic problem is described in the appendix. 


\section{Chapter 3}

\section{Conductance Across One Boundary in}

\section{D}

We start building our intuition by studying coherent thermal impedance matching between two dissimilar 1D materials by controlling the properties of a single mass (Fig. 3.1a) or spring (Fig. 3.1b) in between. This toy model presents a starting point to understand ballistic contributions to TBC by important factors already identified in the literature, like interfacial impurities, mixing, defects, chemistry or bond strength $[17,20,56,57,58,59,51,60]$. In fact, some authors have used this toy model to support their Molecular Dynamic simulation results arguing the increase of TBC with increase of bond strength [61, 62, 63].

The results for coherent, 1-D thermal impedance matching are incredibly diverse. For example, to achieve maximum thermal conductance we want the interfacial impedance to maximize the area under the transmission function, like a broadband filter. Following this criterion, we point out that the best matching interfacial mass $\left(m_{0}\right)$ for the single mass junction (Fig. 3.1a) is the arithmetic mean between the masses of

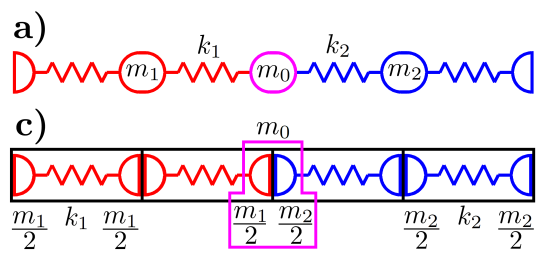

b)
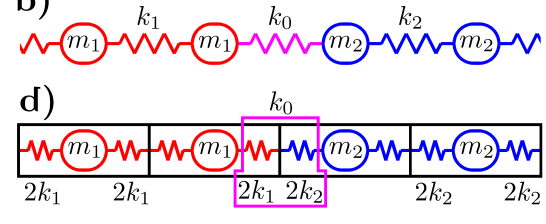

Figure 3.1: a) 1D interface between dissimilar materials with an arbitrary atom in between. b) Optimal coupling between the contacts happens when we can describe the entire heterostructure only in terms of building blocks of individual materials. This decomposition makes the optimal atomic mass the arithmetic mean of its neighbors $m_{0}=m_{1} / 2+m_{2} / 2$. c) Interface with an arbitrary bond in between. d) Maximum thermal conductance occurs when the interfacial spring constant is the harmonic mean of its neighbors $1 / k_{0}=1 / 2 k_{1}+1 / 2 k_{2}$ (recall that a spring half as short is twice as strong), which follows again from a decomposition in terms of blocks of individual materials. 
the contacts [64]. For a single spring junction (Fig. 3.1b), Zhang et al. [65] found that the best matching interfacial spring constant $\left(k_{0}\right)$ is the harmonic mean between the contact springs. When the goal is to achieve maximum phonon transmission around a fixed frequency, our expectations based on our knowledge of optical antireflection coatings posit that unity phonon transmission would require a quarter wave plate with an impedance equal to the geometric mean of its neighbors. In view of these diverse results, our aim here is to motivate these averages qualitatively in terms of the intrinsic physical properties of the junction itself.

The central point of this chapter is that the degree of mismatch at a single atom or bond interface depends on our ability to express the entire heterostructure solely in terms of primitive building blocks on either side. For instance, we find that the optimal mass (Fig. 3.1a) is one that can be decomposed precisely into two half-masses arising from the materials on either side (Fig. 3.1c). This decomposition makes the optimal mass the arithmetic mean of its neighbors, i.e. $m_{0}=m_{1} / 2+m_{2} / 2$. For an analogous decomposition of the spring constant (Fig. 3.1b and d), we find that the optimal spring constant equals the harmonic mean of its neighbors, i.e. $1 / k_{0}=1 / 2 k_{1}+1 / 2 k_{2}$ (Recall that springs in series add like resistances in parallel). Any deviation from those optimal decompositions ("Optimally Coupled Interfaces"-OCI) adds an extra barrier for heat carriers reducing the interfacial transmission.

The thermal conductance for OCI is characterized by the contact induced broadening matrix $\Gamma(\omega)$ extracted from the local vibronic spectrum, which generalizes the concept of acoustic impedance $(Z) . \Gamma$ not only includes non linear dispersion and short-wavelength (atomic) limit effects but its matrix character can account for the different modes or channels available for transport when higher dimensions are considered. Also, this character can include intricate chemical details at the interface, which may greatly affect the transport process as shown recently by Losego et at. [3]. It is worth emphasizing that $\Gamma$ alone is not enough to correctly represent general phonon transport. The broadening's Hilbert transform must be also included in the Green's function to properly account for the sum rule of the local density of states [24].

This generalization not only relates the continuum formalism [66] with the discrete Non-Equilibrium Green's Functions (NEGF) formalism [24, 67, 27, 68, 31], but also provides a way to extrapolate known results based on acoustic impedance to OCI. For instance, we can totally eliminate the interfacial reflection by impedance matching of the $\Gamma$ matrices (more precisely, the projected self-energies, $\Sigma$ ), realized when $\Gamma$ for the central layer equals the geometric mean of its neighbors. The generalization may also allow us to use existing techniques from other engineering fields in phonon engineering. For instance, broadband filter techniques from microwave engineering may be useful to engineer interfaces with maximum thermal conductance.

The chapter begins by explaining the idea of splitting 1D chains into primitive blocks, which define the properties of contacts or semi-infinite chains (Section 3.1). Then, using the block concept, phonon transmission is calculated in section 3.2, where it is also shown that maximum thermal conductance occurs 

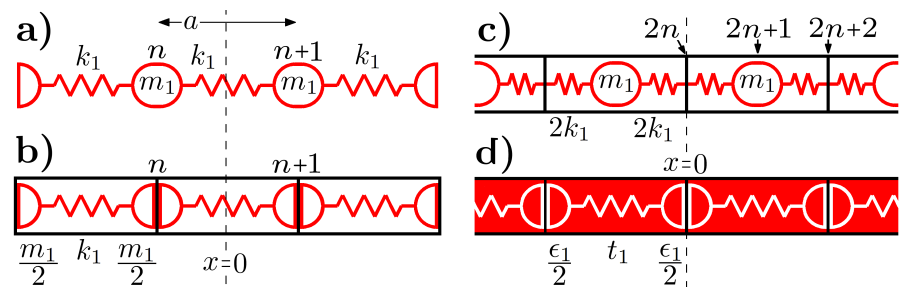

Figure 3.2: a) 1D infinite chain of masses $m_{1}$, separated by $a$, joined by springs with force constant $k_{1}$. b) and c) Same chain separated into different blocks whose boundaries define different contacts. b) Partition into half-mass blocks with half masses across the boundary. c) Partition into half-spring blocks with half springs across the boundary. d) The half-spring blocks can be reinterpreted as half-mass blocks, provided the corresponding mass and spring constant are frequency dependent. The non-white background of the blocks represent this dependence.

when the entire heterostructure can be expressed solely in terms of the building blocks on either side of the interface. These types of interfaces (OCI) are studied and characterized in section 3.3, where it is shown that OCI generalizes an abrupt interface in the continuum limit with $\Gamma$ generalizing $Z$.

\subsection{Block Partition of 1D Chain and Contacts}

An infinite 1D chain of masses coupled by springs (Fig. 3.2a) can be decomposed into different arrays of primitive blocks (Figs. 3.2b and 3.2c ). According to the blocks, different contacts, i.e. semi-infinite chains, can be built from the same homogeneous material. As we will also show, we can equally view a chain of half-spring blocks as a virtual chain of half-mass blocks, provided that the corresponding mass and spring constant are frequency dependent.

Consider a 1D infinite chain of masses $m_{1}$, separated by a distance $a$ and connected by springs with force constant $k_{1}$ (Fig. 3.2a). Newton's equation for the normalized displacement of the $n^{t h}$ atom, $\mu_{n}(t)=u_{n} e^{-i \omega t}$ with dimensions inverse square root of mass, i.e. $[\mu]=\left[M^{-1 / 2}\right]$, is given by

$$
\omega^{2} m_{1} u_{n}=-k_{1} u_{n-1}+2 k_{1} u_{n}-k_{1} u_{n+1}
$$

Plane waves $u_{n}=A e^{i q_{1} n a}$ solve this set of periodic equations. Also, after their substitution on Eq. 3.1 we obtain the dispersion relation

$$
\omega^{2} m_{1}=2 k_{1}-2 k_{1} \cos \left(q_{1} a\right)
$$

which is more commonly written as [69]

$$
\omega=2 \sqrt{\frac{k_{1}}{m_{1}}}\left|\sin \left(\frac{q_{1} a}{2}\right)\right| .
$$


Splitting each mass into its series equivalent $m_{1}=m_{1} / 2+m_{1} / 2$, the chain can be partitioned into blocks with boundaries at the masses, i.e. half-mass blocks (Fig. 3.2b). In this case, Eq. 3.1 and 3.2 can be reorganized to reflect the partition as

$$
\begin{gathered}
\omega^{2}\left(\frac{m_{1}}{2}+\frac{m_{1}}{2}\right) u_{n}=-k_{1} u_{n-1}+2 k_{1} u_{n}-k_{1} u_{n+1} \\
\omega^{2}\left(\frac{m_{1}}{2}+\frac{m_{1}}{2}\right)=2 k_{1}-2 k_{1} \cos \left(q_{1} a\right) .
\end{gathered}
$$

Note that the plane waves solving those equations represent $|A|^{2} N m_{1}$ propagating phonons of energy $\hbar \omega(N$ is the number of atoms in the chain) and carry a thermal current given by [27]

$$
J=\hbar k_{1} \sin \left(q_{1} a\right)|A|^{2}=\hbar \frac{\Gamma_{1}^{h m}}{2}|A|^{2}=\hbar \omega \frac{m_{1}}{a} v_{g}(\omega)|A|^{2}
$$

where

$$
\Gamma_{1}^{h m}=2 k_{1} \sin \left(q_{1} a\right)
$$

("hm" stands for half-mass) is the non-zero entry of the broadening matrix used in NEGF formalism and $v_{g}(\omega)$ is the frequency dependent phonon group velocity.

Similarly, we can split each spring into its series equivalent $1 / k_{1}=1 / 2 k_{1}+1 / 2 k_{1}$, separating the chain (Fig. 3.2a) into blocks with boundaries at the springs, i.e. half-spring blocks (Fig. 3.2c). The latter system is described by

$$
\begin{gathered}
\omega^{2} m_{1} u_{2 n+1}=-2 k_{1} u_{2 n}+2\left(k_{1}+k_{1}\right) u_{2 n+1}-2 k_{1} u_{2 n+2}, \\
0=-2 k_{1} u_{2 n-1}+2\left(k_{1}+k_{1}\right) u_{2 n}-2 k_{1} u_{2 n+1} .
\end{gathered}
$$

Solving for $u_{2 n}$ from Eq. 3.9 and for $u_{2 n+2}$ from Eq. 3.9 substituting $n$ by $n+1$, and replacing those solutions into Eq. 3.8 yields Eq. 3.1. More interestingly, solving for $u_{2 n+1}$ from Eq. 3.8 and for $u_{2 n-1}$ from Eq. 3.8 (substituting $n$ by $n-1$ ) and replacing into Eq. 3.9, results in an equation similar to Eq. 3.4

$$
\omega^{2}\left(\frac{\epsilon_{1}}{2}+\frac{\epsilon_{1}}{2}\right) u_{2 n}=-t_{1} u_{2 n-2}+2 t_{1} u_{2 n}-t_{1} u_{2 n+2}
$$

where

$$
\epsilon_{1}=\frac{m_{1}}{1-\frac{\omega^{2}}{\omega_{c 1}^{2}}}, \quad t_{1}=\frac{k_{1}}{1-\frac{\omega^{2}}{\omega_{c 1}^{2}}}
$$

are frequency dependent coefficients and the cut off frequency is given by $\omega_{c 1}=2 \sqrt{k_{1} / m_{1}}$. In other words, the half-spring block chain can be interpreted as a virtual half-mass block chain having frequency dependent 
masses and springs.

This analogy permits the extrapolation of algebraic treatments, like NEGF, from half-mass block to virtual half-mass block chains. For instance, plane waves describing the displacement at the boundaries of half-spring blocks $u_{2 n}=A e^{i q_{1} 2 n \frac{a}{2}}=A e^{i q_{1} n a}$ satisfy the dispersion relation

$$
\omega^{2}\left(\frac{\epsilon_{1}}{2}+\frac{\epsilon_{1}}{2}\right)=2 t_{1}-2 t_{1} \cos \left(q_{1} a\right)
$$

and carry a thermal current

$$
J=\hbar t_{1} \sin \left(q_{1} a\right)|A|^{2}=\hbar \frac{\Gamma_{1}^{h s}}{2}|A|^{2}=\hbar \omega \frac{\epsilon_{1}}{a} v_{g}(\omega)|A|^{2}
$$

with

$$
\Gamma_{1}^{h s}=2 t_{1} \sin \left(q_{1} a\right)
$$

("hs" stands for half-spring) the non-zero entry of the broadening matrix used in NEGF formalism for the virtual chain.

Although the same infinite chain or bulk material can be built from any block, different contacts are created from different blocks. Indeed, the block choice defines the edge of the contact, the positions in space described by displacement plane waves $A e^{i q n a}$ (block boundaries) and more importantly the thermal current carried by those waves. One striking example of the difference between half-mass and half-spring contacts arises when we connect them together. A phonon impinging on such an interface has non-zero probability of reflection, unlike a phonon propagating in a single block chain. Note that this interface mimics a growth defect in a $1 \mathrm{D}$ crystal.

\subsection{Transmission Using Blocks}

Since a set of phonons of equal energy propagating in a crystal are well represented by plane waves, the transmission probability of phonons impinging at an interface can be calculated from the ratio between the thermal currents carried by the transmitted and incident waves. This section presents phonon transmission calculations using the block concept to simplify the process. It is shown that maximum transmission at every frequency, and therefore maximum thermal conductance, happens when the entire heterostructure can be expressed solely in terms of building blocks on either side. This idea is equivalent to choosing the interfacial atomic mass as the arithmetic mean or the interfacial spring constant as the harmonic mean of its neighbors. 


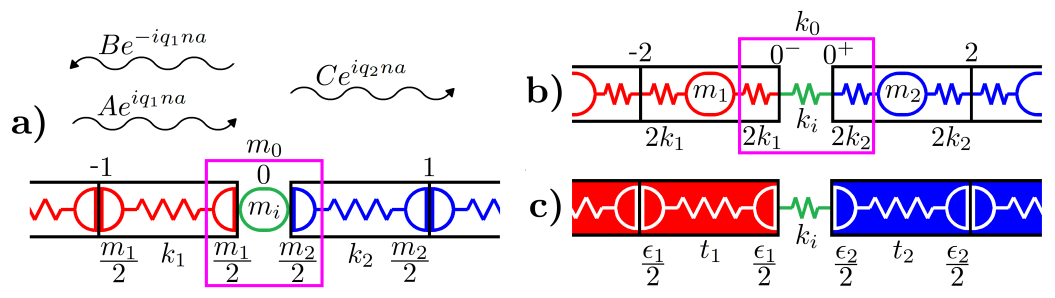

Figure 3.3: Decomposition of interfaces into blocks for transmission calculations. An upper bound for transmission happens when the impurity atom $m_{i}$ or bond $1 / k_{i}$ are zero. a) Atom junction interface split into half-mass blocks. (b) Bond junction interface split into half-spring or (c) virtual half-mass blocks. In this case $u_{2 n}=A e^{i q_{1} n a}+B e^{i q_{1} n a}$.

\subsubsection{Interface with Mass Junction}

Imagine chopping the materials of Fig. 3.1a into half-mass blocks and the interfacial mass into a series equivalent that completes the contacts' blocks plus some residual mass $m_{i}$, i.e.

$$
m_{0}=\frac{m_{1}}{2}+m_{i}+\frac{m_{2}}{2}
$$

(Fig. 3.3a). Assuming incident, reflected and transmitted plane wave solutions, transmission is given by the ratio of transmitted over incident thermal currents

$$
T=\frac{J_{t}}{J_{i}}=\frac{\Gamma_{2}^{h m}}{\Gamma_{1}^{h m}}\left|\frac{C}{A}\right|^{2}
$$

The relationship between $A$ and $C$ is found by substituting the assumed solution

$$
u_{n}= \begin{cases}A e^{i q_{1} n a}+B e^{-i q_{1} n a} & n \leq 0 \\ C e^{i q_{2} n a} & n \geq 0\end{cases}
$$

into Newton's equation at the interface of Fig. 3.3a $(n=0)$

$$
\omega^{2}\left(\frac{m_{1}}{2}+m_{i}+\frac{m_{2}}{2}\right) u_{0}=-k_{1} u_{-1}+\left(k_{1}+k_{2}\right) u_{0}-k_{2} u_{1}
$$

This process is simplified using Eq. 3.5, noting that the real part of the right hand side of Eq. 3.16 exactly cancels $\omega^{2}\left(m_{1}+m_{2}\right) u_{0} / 2$, which yields

$$
\omega^{2} m_{i} u_{0}=i(A-B) \frac{\Gamma_{1}^{h m}}{2}-i C \frac{\Gamma_{2}^{h m}}{2} .
$$


Combining this result with the fact that $u_{0}=A+B=C$, the transmission $T$ is found to be a Breit-Wigner form

$$
T\left(\omega, m_{i}\right)=\frac{4 \Gamma_{1}^{h m} \Gamma_{2}^{h m}}{4 \omega^{4} m_{i}^{2}+\left(\Gamma_{1}^{h m}+\Gamma_{2}^{h m}\right)^{2}},
$$

with $m_{i}$ being the deviation of the interfacial mass $m_{0}$ from the arithmetic mean between the contact masses (Eq. 3.14).

Note that the largest possible transmission for every $\omega$ is obtained when $m_{i}=0$. This choice maximizes the thermal current flowing across the interface and the thermal conductance of the system. That is,

$$
\begin{aligned}
I_{0} & =\int d \omega \frac{\hbar \omega}{2 \pi} T(\omega, 0)\left(N_{1}-N_{2}\right) \\
& \geq \int d \omega \frac{\hbar \omega}{2 \pi} T\left(\omega, m_{i}\right)\left(N_{1}-N_{2}\right)=I_{m_{i}} .
\end{aligned}
$$

In this particular case, the system becomes equivalent to an abrupt interface between contacts built with half-mass blocks, which is referred as an "Optimally Coupled Interface" (OCI). When $m_{i} \neq 0$, transmission decreases (for all $\omega$ ) so $m_{i}$ can be associated with an extra barrier lowering the thermal conductance. Specifically a delta scattering center like a single point impurity or defect at the interface.

\subsubsection{Interface with Spring Junction}

Imagine now chopping the contacts of Fig. 3.1b into half-spring blocks and the interfacial spring into its series equivalent

$$
\frac{1}{k_{0}}=\frac{1}{2 k_{1}}+\frac{1}{k_{i}}+\frac{1}{2 k_{2}}
$$

(Fig. 3.3b). Assuming incident, reflected and transmitted plane wave solutions at the blocks' boundaries, transmission is given by the ratio of transmitted over incident thermal currents

$$
T=\frac{J_{t}}{J_{i}}=\frac{\Gamma_{2}^{h s}}{\Gamma_{1}^{h s}}\left|\frac{C}{A}\right|^{2} .
$$

The relationship between $A$ and $C$ is found by substituting the assumed solution

$$
u_{2 n}= \begin{cases}A e^{i q_{1} n a}+B e^{-i q_{1} n a} & n<0 \text { and } n=0^{-} \\ C e^{i q_{2} n a} & n>0 \text { and } n=0^{+}\end{cases}
$$


into Newton's equation at the interface $\left(n=0^{-}\right.$and $\left.n=0^{+}\right)$for the virtual chain (Fig. 3.3c)

$$
\begin{gathered}
\omega^{2} \frac{\epsilon_{1}}{2} u_{0^{-}}=-t_{1} u_{-2}+\left(t_{1}+k_{i}\right) u_{0^{-}}-k_{i} u_{0^{+}} \\
\omega^{2} \frac{\epsilon_{2}}{2} u_{0^{+}}=-k_{i} u_{0^{-}}+\left(k_{i}+t_{2}\right) u_{0^{+}}-t_{2} u_{2} .
\end{gathered}
$$

This process is simplified using Eq. 3.11, noting that the real part of $t_{1}\left(u_{0^{-}}-u_{-2}\right)$ from Eq. 3.21 and $t_{2}\left(u_{0^{+}}-u_{2}\right)$ from Eq. 3.22 exactly cancel $\omega^{2} \epsilon_{1} u_{0^{-}} / 2$ and $\omega^{2} \epsilon_{2} u_{0^{+}} / 2$ respectively, which yields

$$
0=k_{i} u_{0^{-}}-k_{i} u_{0+}+i(A-B) \frac{\Gamma_{1}^{h s}}{2}
$$

and

$$
0=-k_{i} u_{0^{-}}+k_{i} u_{0+}-i C \frac{\Gamma_{2}^{h s}}{2}
$$

Combining these two results with $u_{0^{-}}=A+B$ and $u_{0^{+}}=C$, the transmission $T$ is given by

$$
T\left(\omega, k_{i}^{-1}\right)=\frac{4 \Gamma_{1}^{h s} \Gamma_{2}^{h s}}{\frac{1}{4 k_{i}^{2}}\left(\Gamma_{1}^{h s} \Gamma_{2}^{h s}\right)^{2}+\left(\Gamma_{1}^{h s}+\Gamma_{2}^{h s}\right)^{2}},
$$

with $k_{i}^{-1}$ measuring the deviation of the interfacial spring $k_{0}$ from the harmonic mean between the contact springs (Eq. 3.19).

Note that the largest possible transmission for every $\omega$ is obtained when $k_{i}^{-1}=0$. This choice maximizes the thermal current flowing across the interface and the thermal conductance of the system. In that case, the system also becomes equivalent to an abrupt interface between contacts built with half-spring blocks, which is also referred as an "Optimally Coupled Interface". When $k_{i}^{-1} \neq 0$, transmission (for all $\omega$ ) and thermal conductance decrease, so that $k_{i}^{-1}$ can be associated with an extra barrier at the interface.

\subsection{Optimally Coupled Interfaces}

An "Optimally Coupled Interface" (OCI) is an abrupt interface between half-mass or half-spring block contacts, which was proven equivalent to the single atomic or bonding interface with maximum possible transmission or thermal conductance (Section 3.2). This section shows that an OCI can be thought of as a step barrier for phonons responsible for the scattering due to a change in propagation medium. On the other hand, a non-OCI is represented by the same step barrier plus an extra barrier caused by a deviation from the optimal case $\left(m_{i} \neq 0\right.$ or $\left.k_{i}^{-1} \neq 0\right)$. The extra barrier decreases thermal conductance and can be associated with additional scattering mechanisms at the interface, such as impurities, mixing or dislocations. 
This section presents a useful way to visualize transmission in OCI from the contact broadenings and extends the concept of OCI to abrupt junctions between contacts built with different types of blocks.

The section also shows that OCI generalizes an abrupt interface in the continuum limit without the long wavelength constraint. Moreover, the bulk property $Z$ (acoustic impedance) is generalized by the contact property $\Gamma$ (broadening), which unlike $Z$ includes the atomistic details of the contact's edge and the non-linear effects of phonon dispersion. This analogy shows a way to extrapolate previous results for interfaces in the continuum limit to the discrete limit by replacing abrupt interfaces with OCI and $Z$ with $\Gamma$. For instance, the result of a thermal antireflection coating for a quarter wave length plate is obtained when the plate broadening equals the geometric mean of the individual contact broadenings.

\subsubsection{Continuous vs. Discrete Limit}

The continuous medium approximation assumes that the wavelengths of interest are large enough $(\lambda>>a)$ so that the atomistic details of the media are ignorable, the dispersion is linear and the group velocity is constant. Within this approximation, the scattering problem at an interface (Table 3.1-a) is solved by assuming incident, reflected and transmitted plane waves solutions and imposing boundary conditions on them to guarantee the validity of the wave equation at the interface. These conditions are nicely simplified introducing the concept of acoustic impedance (Table 3.1-b and $\mathbf{c}[66]$ )

$$
Z=\rho v_{g}=\frac{m}{a}\left(a \sqrt{\frac{k}{m}}\right)=\sqrt{k m}
$$

From them, the ratio of the wave amplitudes is calculated and then transmission is found from the ratio of transmitted over incident energy currents (Table 3.1-e). Scattering at these interfaces can be connected with medium mismatch using the reflection coefficient

$$
R=1-T=\left(\frac{Z_{1}-Z_{2}}{Z_{1}+Z_{2}}\right)^{2}
$$

which vanishes only when $Z_{1}|A|^{2}=Z_{2}|A|^{2}$. In words, if plane waves of the same amplitude do not carry the same energy current in both media then some energy has to be reflected. That is, the scattering is solely caused by mismatch of the medium properties.

When the long wavelength constraint is relaxed, the frequency dependent group velocity, the cut-off frequency and the atomistic details of the interface affect the transmission (All included in calculations in section 3.2). Nevertheless, particularizing the transmission calculation in section 3.2 to the optimal case ( $m_{i}=0$ for mass junction and $k_{i}^{-1}=0$ for spring junction) displays the resemblance between an OCI and 


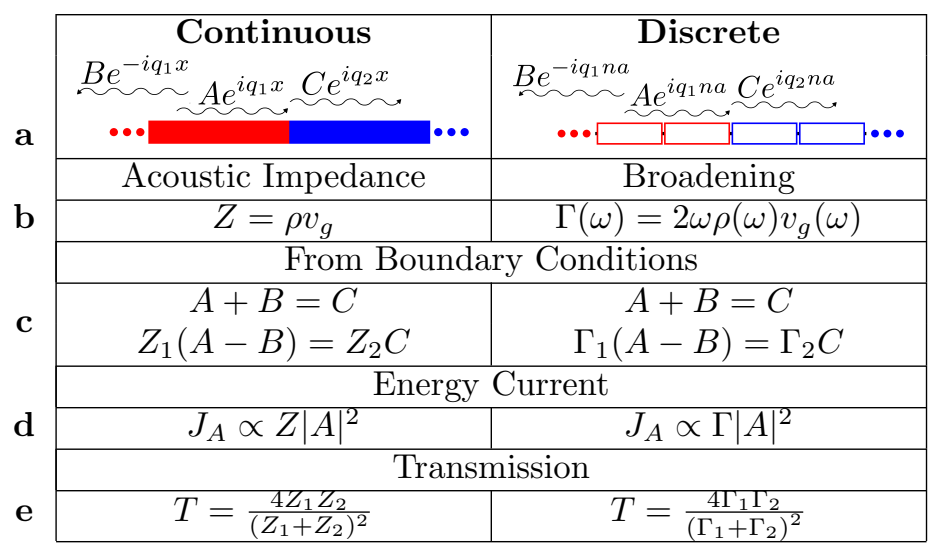

Table 3.1: Parallel between an abrupt interface in the continuum limit and an Optimally Coupled Interface. The resembling suggests that the contact induced broadening matrix $\Gamma$ generalizes the concept of acoustic impedance $(Z)$ to the nonlinear phonon dispersion as well as the short-wavelength (atomic) limit. For $Z$, $\rho=m / a$. For $\Gamma, \rho=m / a$ or $\rho=\epsilon / a$ according to the block choice. Note that the matrix character of $\Gamma$ can account for all the conduction modes available in higher dimensions, for interactions beyond first neighbor and for tensorial properties of materials.

an abrupt interface in the continuum limit (Table 3.1). This suggests that OCI generalizes the continuous interface with $\Gamma$ playing the role of acoustic impedance $Z$. Note that in the long wavelength limit $\Gamma \rightarrow 2 \omega Z$ (for both $\Gamma^{h m}$ and $\Gamma^{h s}$ ) and we recover the transmission result in terms of $Z$

$$
T=\frac{4 \Gamma_{1} \Gamma_{2}}{\left(\Gamma_{1}+\Gamma_{2}\right)^{2}} \stackrel{\lambda>>a}{\longrightarrow} \frac{4 Z_{1} Z_{2}}{\left(Z_{1}+Z_{2}\right)^{2}}
$$

Similar to the continuum limit, scattering in OCI is solely due to contact mismatch. That is, if plane waves of the same amplitude do not carry the same energy current in both contacts then some energy has to be reflected. The subtle difference from medium to contact reflects the fact that unlike $Z, \Gamma$ is a contact property which ultimately depends on the block choice and carry information about the contact's edge.

After identifying contact mismatch scattering with the transmission functional defining OCI (Table 3.1-e), the extra term decreasing the transmission in Eq. 3.18 or 3.25 is associated with an additional source of scattering at the interface. Following this train of ideas, an OCI is represented by a frequency dependent step barrier for phonons responsible for contact mismatch scattering while a non OCI is represented by the same step barrier plus an extra barrier that decreases transmission and can be associated with impurities, mixing or dislocations (Fig. 3.4).

Note that if the blocks don't have a 2-fold rotation symmetry around their centers, e.g. the masses in a half-mass block are not equal but still their equivalent mass equals $m_{1}$, the simplifications in Table 3.1-c are not possible for every $\omega$. That is, abrupt interfaces between contacts built with non-symmetric blocks do not resemble the equations in the continuum limit. 


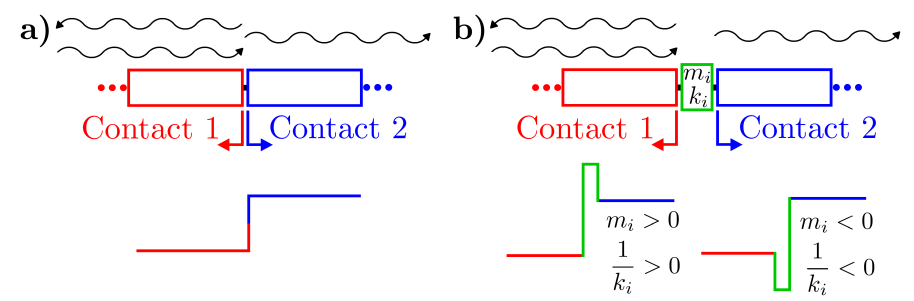

Figure 3.4: a) Optimally Coupled Interface and its representation as a single barrier for phonons, which is responsible for the scattering due to propagating waves changing medium. b) Non OCI and its representation as a step barrier plus an extra barrier caused by a deviation from the optimal case. This extra barrier decreases thermal conductance and can be associated with impurities, mixing or defects at the interface.

\subsubsection{Characteristics of OCI}

OCI transmission can be visualized from the contact broadenings using the reflection coefficient,

$$
R=1-T=\left|\frac{\Gamma_{1}-\Gamma_{2}}{\Gamma_{1}+\Gamma_{2}}\right|^{2}
$$

Unity transmission $\left(T\left(\omega_{*}\right)=1\right)$ is obtained when $\Gamma$ 's match $\left(\Gamma_{1}\left(\omega_{*}\right)=\Gamma_{2}\left(\omega_{*}\right)\right)$ at a particular frequency $\omega_{*}$. That is, a phonon with energy $\hbar \omega_{*}$ does not see the interface. Null transmission $(T(\omega)=0)$ is obtained if any of the $\Gamma$ 's becomes 0 or imaginary. This defines a cut off frequency $\omega_{c}$ over which phonons do not propagate in the contact. Also, note that the frequency dependence of $\Gamma$ disallows the possibility of matching different contacts at every $\omega$, making scattering unavoidable.

When a contact is built with half-mass blocks, $\Gamma_{1}$ from Eq. 3.7 can be rewritten using the dispersion relation as

$$
\Gamma_{1}^{h m}=2 k_{1} \sqrt{1-\left(1-\frac{2 \omega^{2}}{\omega_{c 1}^{2}}\right)^{2}}
$$

with $\omega_{c 1}=2 \sqrt{k_{1} / m_{1}}$. This concave function vanishes at $\omega=0$ and $\omega=\omega_{c_{1}}$ and has a maximum value $2 k_{1}$ at frequency $\omega_{c_{1}} / \sqrt{2}$ (Fig. 3.5a). With this function in mind, transmission of an OCI between half-mass block contacts (Fig. 3.5d) can be visualized from a plot of the real part of both contact broadenings (Fig. 3.5a) and Eq. 3.29. Figs. 3.5a and 3.5d show the case when the contacts match at a particular frequency $\omega_{*}$, i.e. when $T\left(\omega_{*}\right)=1$. This is only possible if $\Gamma$ 's intersect, which requires $k_{1}<k_{2}$ and $\omega_{c 1}>\omega_{c 2}$ or $k_{1}>k_{2}$ and $\omega_{c 1}<\omega_{c 2}$. The intersection frequency $\omega_{*}$ is found by equating $\Gamma_{1}\left(\omega_{*}\right)=\Gamma_{2}\left(\omega_{*}\right)$ as

$$
\omega_{*}^{2}= \begin{cases}\frac{4\left(m_{1} k_{1}-m_{2} k_{2}\right)}{m_{1}^{2}-m_{2}^{2}} & \text { if } m_{1} \neq m_{2} \\ {\left[0, \min \left(\omega_{c_{1}}, \omega_{c 2}\right)\right]} & \text { if } m_{1}=m_{2} \text { and } k_{1}=k_{2} \\ \text { never } & \text { if } m_{1}=m_{2} \text { and } k_{1} \neq k_{2}\end{cases}
$$




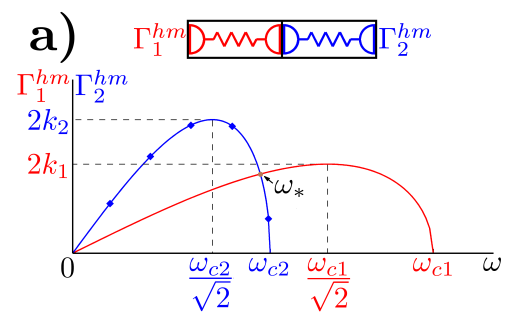

c) $\Gamma_{1}^{h s}=-1-W-\left(\Gamma_{2}^{h m}\right.$
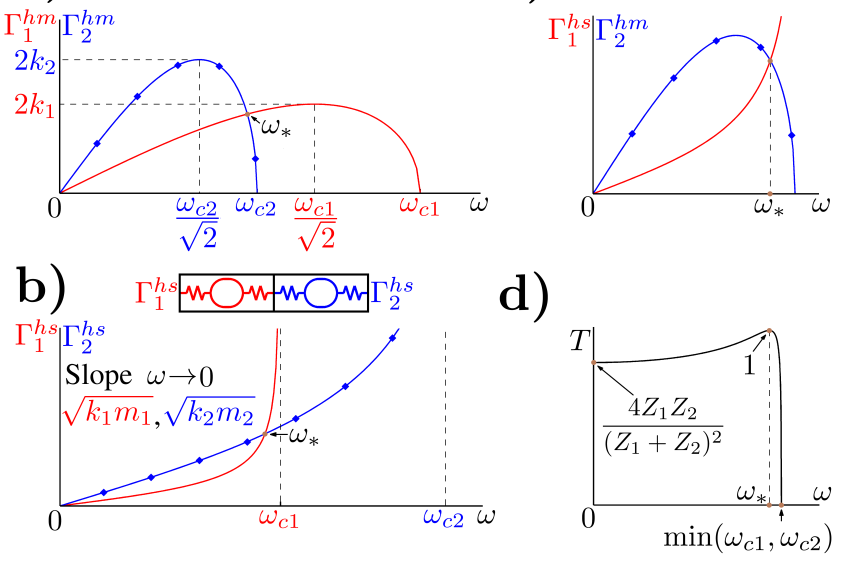

Figure 3.5: Transmission of OCI visualized from the broadening of the contacts $\left(\Gamma_{1}\right.$ and $\left.\Gamma_{2}\right)$. a), b) and c) $\Gamma$ 's for half-mass contacts, half-spring contacts and half-spring, half-mass contacts respectively. The dotted lines represent $\Gamma_{2}$. d) Characteristic transmission function for all the cases before. At the particular frequency $\omega_{*}$, where $\Gamma$ s intersect each other, transmission becomes unity and the materials match.

Note that when acoustic impedances match the intersection frequency is zero, which makes sense since in the continuous regime any wave looks like a zero frequency wave. When the contacts do not match or $\Gamma$ 's never intersect, the transmission never reaches unity. This happens if $k_{1}>k_{2}$ and $\omega_{c 1}>\omega_{c 2}$ or $k_{1}<k_{2}$ and $\omega_{c 1}<\omega_{c 2}$. Another interesting case occurs when the cut-off frequencies are equal, which makes $\Gamma_{1} \propto \Gamma_{2}$ and therefore transmission is constant.

When a contact is built with half-springs blocks, $\Gamma_{1}$ from Eq. 3.13 can be rewritten using the dispertion relation as

$$
\Gamma_{1}^{h s}=4 k_{1} \sqrt{\frac{\omega^{2}}{\omega_{c_{1}}^{2}-\omega^{2}}} .
$$

This convex function vanishes at $\omega=0$, is $\infty$ at $\omega=\omega_{c_{1}}$ and has a slope at $\omega=0$ of $2 \sqrt{k_{1} m_{1}}$ (Fig. 3.5b). Similar to the former case, Fig. 3.5b and d show the case when the contacts match, which is only possible if $k_{1} m_{1}>k_{2} m_{2}$ and $\omega_{c 1}>\omega_{c 2}$ or $k_{1} m_{1}<k_{2} m_{2}$ and $\omega_{c 1}<\omega_{c 2}$. The intersection frequency $\omega_{*}$ is found equating $\Gamma_{1}\left(\omega_{*}\right)=\Gamma_{2}\left(\omega_{*}\right)$ as

$$
\omega_{*}^{2}= \begin{cases}4 \frac{k_{1} k_{2}}{m_{1} m_{2}} \frac{\left(m_{1} k_{1}-m_{2} k_{2}\right)}{k_{1}^{2}-k_{2}^{2}} & \text { if } k_{1} \neq k_{2} \\ {\left[0, \min \left(\omega_{c_{1}}, \omega_{c 2}\right)\right]} & \text { if } k_{1}=k_{2} \text { and } m_{1}=m_{2} \\ \text { never } & \text { if } k_{1}=k_{2} \text { and } m_{1} \neq m_{2}\end{cases}
$$

The contacts do not match if $k_{1} m_{1}<k_{2} m_{2}$ and $\omega_{c 1}>\omega_{c 2}$ or $k_{1} m_{1}>k_{2} m_{2}$ and $\omega_{c 1}<\omega_{c 2}$ and transmission is constant if $\omega_{c 1}=\omega_{c 2}$. 


\subsubsection{Other OCI}

Since the key to obtain an OCI is the use of symmetric blocks, one can imagine that an abrupt interface between contacts built with different types of block is also an OCI. In fact this is shown starting from a more general interface where two parameters $m_{0}$ and $k_{0}$ can be varied (Fig. 3.6). Using the block concept to define the contacts and the impurities, phonon transmission is found to be

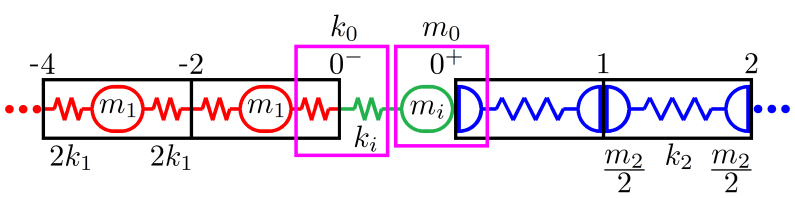

Figure 3.6: 1D interface between dissimilar materials with an arbitrary bond and atom in between. An OCI between half-spring and half-mass contacts arises when $m_{i}=0$ and $1 / k_{i}=0$. However, this OCI is an upper bound only for thermal conductance of systems in which $m_{0}$ vary arbitrarily and $1 / k_{i}=0$ or reciprocally. When $k_{0}$ and $m_{0}$ can vary together, interferences enter to the picture and the upper bound is lost. Interface with varying mass $m_{0}=m_{i}+\frac{m_{2}}{2}$ and spring $\frac{1}{k_{0}}=\frac{1}{k_{i}}+\frac{1}{2 k_{1}}$. The $1 \mathrm{D}$ chain modeled is obtained by combining the springs and masses when possible.

$$
T=\frac{\Gamma_{1}^{h s} \Gamma_{2}^{h m}}{\left[\omega^{2} m_{i}+\frac{1}{k_{i}} \frac{\Gamma_{1}^{h s}}{2} \frac{\Gamma_{2}^{h m}}{2}\right]^{2}+\left[\left(\frac{\Gamma_{1}^{h s}}{2}+\frac{\Gamma_{2}^{h m}}{2}\right)-\omega^{2} \frac{m_{i}}{k_{i}} \frac{\Gamma_{1}^{h s}}{2}\right]^{2}}
$$

From this system, two single junction interfaces can be defined by setting $k_{i}^{-1}$ (or $m_{i}$ ) to zero and letting $m_{0}\left(\right.$ or $\left.k_{0}\right)$ vary. The cancelations in the denominator of Eq. 3.32 expose an OCI when $m_{i}=0$ (or $\left.k_{i}^{-1}=0\right)$ which is an upper bound of thermal conductance for any other choice of $m_{i}$ (or $k_{i}^{-1}$ ). Similar to the cases in the last subsection, plotting $\Gamma_{1}^{h s}$ and $\Gamma_{2}^{h m}$ reflects some transmission characteristics (Fig. 3.5c and 3.5d). If the system is studied as a whole and both impurities do not vanish, the transmission of the OCI is not necessarily an upper bound for every $\omega$. This does not contradict the previous definition of OCI because the interface allows two parameters to vary.

\subsubsection{Extrapolation Example: Beyond Single Interfaces}

In subsection 3.3.1 we showed that OCIs generalize continuous interfaces to the discrete limit, which allows the extrapolation of known results between limits by changing interfaces with OCI and acoustic impedance $Z$ with broadening $\Gamma$. This analogy may provide a way to endow phonon engineering with existing design criteria from other engineering fields. For instance, broadband filter techniques from microwave engineering may be useful to engineer interfaces with maximum thermal conductance. As an example of the generalization, let us consider a system consisting of two mediums sandwiching a third one with impedances $Z_{1}, Z_{2}$ and $Z_{0}$ respectively. Recall that reflection is eliminated when the coupling medium has length of a quarter wavelength 


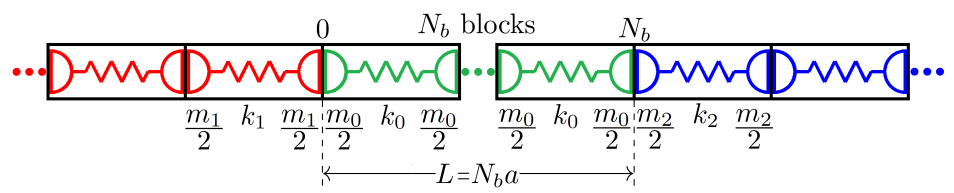

Figure 3.7: System consisting of two mediums sandwiching a third one characterized by the broadenings $\Gamma_{1}$, $\Gamma_{2}$ and $\Gamma_{0}$ respectively. Similar to the antireflection coating condition, transmission is unity when $L=\lambda / 4$ and $\Gamma_{0}=\sqrt{\Gamma_{1} \Gamma_{2}}$.

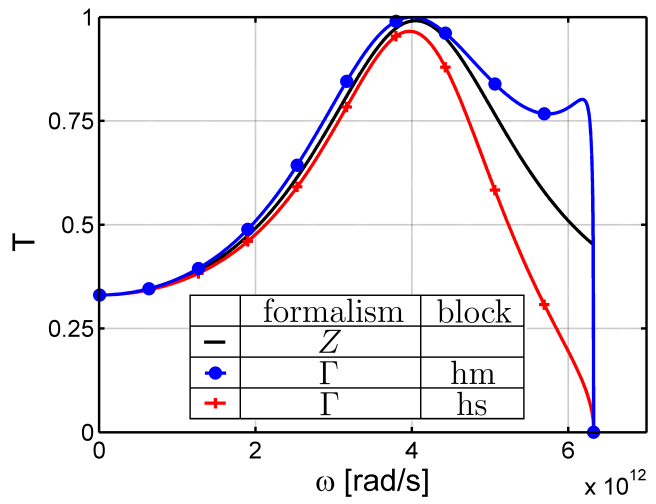

Figure 3.8: Comparison of the transmission function predicted by Eq. 3.33 (dotted line) and its counterpart with the long wavelength constraint, i.e. Eq 3.33 replacing $\Gamma$ with $Z$ (solid line). The system consists only of half-mass blocks (Fig. 3.7) with $m_{1}=10^{-26} \mathrm{~kg}, m_{2}=10^{-24} \mathrm{~kg}, k_{1}=k_{2}=10 \mathrm{~N} / \mathrm{m}$ and $N_{b}=3 . m_{0}$ and $k_{0}$ were chosen to guarantee $T=1 @ \omega=4 \times 10^{12} \mathrm{rad} / \mathrm{s}$, i.e. $m_{0}=1.1779 \times 10^{-25} \mathrm{~kg}$ and $k_{0}=7.0338 \mathrm{~N} / \mathrm{m}$. The line with crosses represents the transmission of a system consisting only of half-spring blocks with the same parameters.

and impedance $Z_{0}=\sqrt{Z_{1} Z_{2}}$. Applying the extrapolation rules to the known solution [66], the system turns into Fig. 3.7 and its transmission is given by (the result can also be obtained following the process in section

$$
T=\frac{4 \frac{\Gamma_{1}}{\Gamma_{2}}}{\left(\frac{\Gamma_{1}}{\Gamma_{2}}+1\right)^{2} \cos ^{2}\left(q_{0} L\right)+\left(\frac{\Gamma_{1}}{\Gamma_{0}}+\frac{\Gamma_{0}}{\Gamma_{2}}\right)^{2} \sin ^{2}\left(q_{0} L\right)},
$$

with $\Gamma$ 's defined according to the block choice in each particular region. Similar to the antireflection condition, transmission is unity when $L=\lambda / 4$ and $\Gamma_{0}=\sqrt{\Gamma_{1} \Gamma_{2}}$.

Unlike the impedance formalism, i.e. Eq 3.33 replacing $\Gamma$ with $Z$, the broadening formalism (Eq. 3.33) includes the effects of non linear dispersion and atomistic details. A comparison of the transmission predicted by both formalisms is shown in Fig. 3.8. Note that as the frequency increases and the non linearity of the dispersion becomes important, the transmission functions separate from each other. Also note that different atomistic details at the interface, defined by our block choice, generate different transmission functions. 


\subsection{Summary of Contributions}

1. Show that $\Gamma$ generalizes the acoustic impedance $Z$ to the nonlinear phonon dispersion as well as the short-wavelength (atomic) limit. This relates the continuum wave formalism with any type of discrete NEGF formalism, including electron and phonon NEGF.

2. Show that the interfaces with maximum conductance (with junction mass equal to the AM of the contact masses and junction spring constant equal to the harmonic mean of the contacts spring constants) are discrete abrupt interfaces. This combined with the point before, provides a way to extrapolate previous results based on acoustic impedance to combinations of abrupt discrete interfaces. For instance, broadband filter techniques from microwave engineering may be useful to engineer interfaces with maximum thermal conductance. 


\section{Chapter 4}

\section{Conductance Across Many}

\section{Boundaries in 1D}

In this chapter we explain how to choose the mass, spring constant and length $(m, k, L)$ of a single or several junction materials to maximize the average of phonon transmission over frequency $\langle T\rangle_{\omega}$, which is equivalent to maximizing the thermal conductance of 1D chains (Fig. 4.1). Our analysis extends the acoustic impedance to nonlinear dispersions using the contact-induced broadening matrix $\Gamma$, obtained from the imaginary part of the self-energy matrix in the Non-Equilibrium Green's Function (NEGF) formalism [6].
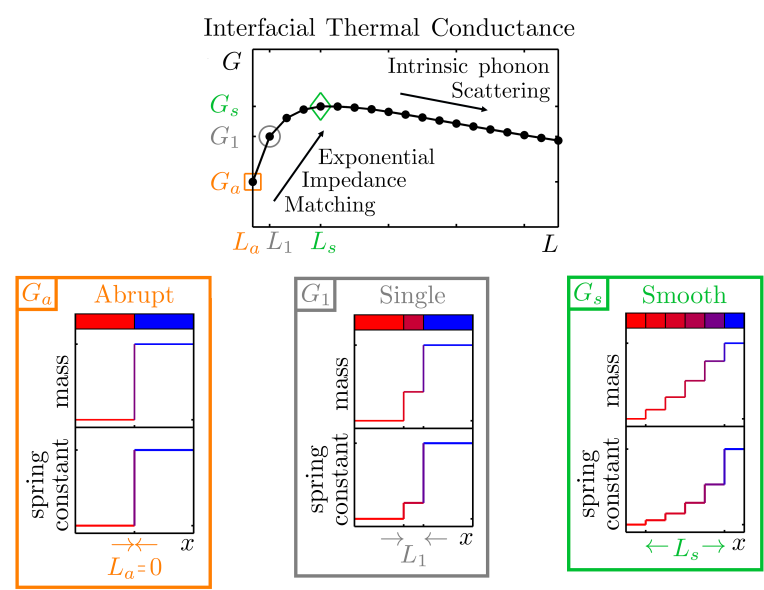

Figure 4.1: Interfacial thermal conductance is maximized by inserting a graded junction with an exponentially varying "impedance" $\Gamma_{n}=\Gamma_{l} e^{\zeta n}$ (Eq. 4.18). However as the length between the contacts increases, the conductance gained by "impedance" matching is dominated by losses due to other scattering mechanisms.

For a single junction material, we find that the desirable broadening is the Geometric Mean (GM) of the contact broadenings. In the coherent limit, this choice maximizes the resonance peaks of the transmission 
function (Sec. 4.2), while in the incoherent limit it minimizes the sum of interfacial resistances (Sec. 4.3). To satisfy the GM condition we need (1) to choose the acoustic impedance as the GM of the contact impedances to favor low frequency phonons and (2) to set the cut-off frequency close to the minimum of the contact cut-off frequencies to favor high frequecy phonon and reduce the spillage of states into the tunelling regime (Fig. 4.3a). In Sec. 4.2, those sub conditions are translated into rules to choose the junction mass, spring constant, sound velocity, Debye temperature or density of states. As the junction becomes a single atom or bond, the resonances are pushed outside the allowed frequency interval and the GM condition transforms to the Arithmetic Mean (AM) of the masses or the Harmonic Mean (HM) of the spring constants of the contacts.

For multi-junction materials, the GM condition evolves into an exponentially varying broadening, since the GM of two quantities stays near the lower partner. That variation generates a broadband anti-reflection coating in the coherent limit (Fig. 4.4b Sec. 4.4) and minimizes the sum of resistances between dissimilar materials in the incoherent limit (Sec. 4.5). In this way, thermal conductance is pushed to the maximum physical limit allowed by the contacts as the number of junctions increases. Finally, we discuss the trade-off between increasing conductance by adding junctions to improve the matching of the contacts and decreasing conductance as a result of enlarging the interface and adding intrinsic scattering (Fig. 4.1).

The results of this chapter can be used for modeling thermal conductance of Self-Assembled Monolayers (SAMs) and for extending 1D propagation models of long-wavelength phonons in semiconductors [70, 71]. It also provides a solid ground to expolore the 3D maximization problem since a 3D system can be decomposed into decoupled "1D like" subsystems, with the caveat that in each of them the nature of the polarization vectors and their overlap should play a role, depending on the symmetry breaking disorder at the interface. The exponentially varying broadening provides one example of phonon transmission engineering inspired by microwave engineering of broad band filters [72]. Similar analogies can be found for the binomial or Chebyshev matching transformers, which optimize the flatness or bandwidth of the transmission function respectively [72]. We can further envision engineering an ultra narrow transmission that filters particular phonons or an extremely low transmission to improve thermoelectric figure of merit.

\subsection{Methodology}

We start from the definition of thermal conductance in Eq. 2.1. Here we focus on a 1D system with 1st neighbor interactions, where $M$ reduces to one. We further simplify Eq. 2.1 by assuming a temperature larger than the Debye temperature, so the factor $\hbar \omega \partial N / \partial \mathbf{T}$ is almost constant over the allowed frequency spectrum. In this way, $G^{Q} \propto\langle T\rangle_{\omega}$ and maximizing $\langle T\rangle_{\omega}$ maximizes $G^{Q}$ (Table 4.1). 
In spite of our second assumption to simplify $G^{Q}$, our results are not limited to high temperatures. As we explain in Sec. 4.2 and 4.3, the necessity of finding a robust maximum requires us to maximize $\left\langle\langle T\rangle_{\varphi}\right\rangle_{\omega}$ the frequency average of the phase average of $T$. The phase average transforms $T$ into a slow varying function by washing away the interference wiggles. Therefore, for the maximization process we can pull $\langle T\rangle_{\varphi}$ the phase average of $T$ out of the integral and Eq. 2.1 can be approximated by

$$
G^{Q} \approx\left\langle\langle T\rangle_{\varphi}\right\rangle_{\omega} \int_{0}^{\omega_{\max }} \frac{d \omega}{2 \pi} \hbar \omega \frac{\partial N}{\partial \mathbf{T}}
$$

Now that the temperature dependence has been isolated, maximizing $\left\langle\langle T\rangle_{\varphi}\right\rangle_{\omega}$ yields to the more robust maximum of $G^{Q}$. The approximation in Eq. 4.1 breaks down for very low temperatures because the frequency average should be done only over the exited phonons which constitutes a frequency range shorter than $\left[0, \omega_{\max }\right]$. Nevertheless, the material properties that maximize $\left\langle\langle T\rangle_{\varphi}\right\rangle_{\omega}$ only differ from the exact solution for temperatures below $10 \%$ of the Debye temperature.

\begin{tabular}{|c|c|}
\hline$T$ & Coherent transmission \\
\hline$\langle T\rangle_{\omega}$ & Frequency average of $T$ over $\left[0, \omega_{\max }\right]$ \\
\hline$\langle T\rangle_{\varphi}$ & Incoherent transmission or phase average of $T$ \\
\hline$\left\langle\langle T\rangle_{\varphi}\right\rangle_{\omega}$ & Frequency average of the phase average of $T$ \\
\hline
\end{tabular}

Table 4.1: Summery of the notation for the different types of phonon transmission used in the document. The maximum freuquency $\omega_{\max }$ is defined by the minimum cut-off frequency of the contacts.

\subsection{Single material, coherent.}

\section{Geometric Mean and anti-reflection coating.}

Analogous to the quantum mechanical transmission of a particle through a barrier, the phonon transmission function $T$ for the system in Fig. 4.2a is found from the ratio between the incident and transmitted heat currents. After assuming plane waves solutions, imposing boundary conditions at the interfaces and replacing the acoustic impedance $Z$ with the broadening matrix $\Gamma[6], T$ is given by

$$
T=\frac{4 \gamma_{l r}}{\left(\gamma_{l r}+1\right)^{2} \cos ^{2}\left(q_{1} L\right)+\left(\gamma_{l 1}+\gamma_{1 r}\right)^{2} \sin ^{2}\left(q_{1} L\right)}
$$

where the wave vector of the junction $q_{1}$ comes from the dispersion relation, i.e. $\omega=\omega_{c 1}\left|\sin \left(q_{1} a / 2\right)\right|$ with the cut-off frequency $\omega_{c 1}=2 \sqrt{k_{1} / m_{1}}$, and $\gamma_{\alpha \beta}=\Gamma_{\alpha} / \Gamma_{\beta}$. The broadening function $\Gamma_{\alpha}$ for the region $\alpha$ is calculated from an infinite 1D chain of material $\alpha$. This is similar to the way we calculate acoustic impedances for independent regions when solving for the transmission of acoustic vibrations through different media. $\Gamma_{\alpha}$ 
describes how easily vibrations can move from one site to the next in the material and is related with the acoustic impedance through $[67,6]$

$$
\Gamma=2 \omega \rho v_{g}=2 \omega \hat{Z}
$$

with $\rho$ the mass density, $v_{g}$ the frequency dependent group velocity and $\hat{Z}$ the frequency dependent impedance, whose low frequency limit is the acoustic impedance $Z$. Note that in this paper the symbol $Z$ is used to denote the frequency independent acoustic impedance, while $\Gamma$ and all the quantities that depend on $\Gamma$ are frequency dependent. Eq. 4.2 is valid for frequencies smaller than all the cut-off frequencies. When $\omega_{c 1}<\omega<\omega_{c l}, \omega_{c r}$, phonons tunnel through the junction and the cos and sin become their hyperbolic counterparts. When the frequency is larger than the maximum frequency $\omega>\min \left(\omega_{c l}, \omega_{c r}\right)=\omega_{\max }, T=0$ defining the allowed frequency range.

Note that the transmission function $T$ also follows from adding coherently all the wave paths that cross the interface

$$
\begin{aligned}
T=\gamma_{r l}|t|^{2} & =\gamma_{r l}\left|t_{l 1} t_{1 r}+t_{l 1} r_{1 r} r_{1 l} t_{1 r} e^{i 2 q_{1} L}+\cdots\right|^{2} \\
& =\frac{T_{l 1} T_{1 r}}{1+2 \sqrt{R_{1 r} R_{1 l}} \cos \left(2 q_{1} L\right)+R_{1 r} R_{1 l}}
\end{aligned}
$$

where at each abrupt interface, the reflection and transmission coefficients are $r_{\alpha \beta}=\left(\gamma_{\alpha \beta}-1\right) /\left(\gamma_{\alpha \beta}+1\right)$ and $t_{\alpha \beta}=\left(2 \gamma_{\alpha \beta}\right) /\left(\gamma_{\alpha \beta}+1\right)$, and the reflection and transmission are $R_{\alpha \beta}=\left|r_{\alpha \beta}\right|^{2}$ and $T_{\alpha \beta}=\gamma_{\beta \alpha}\left|t_{\alpha \beta}\right|^{2}$.

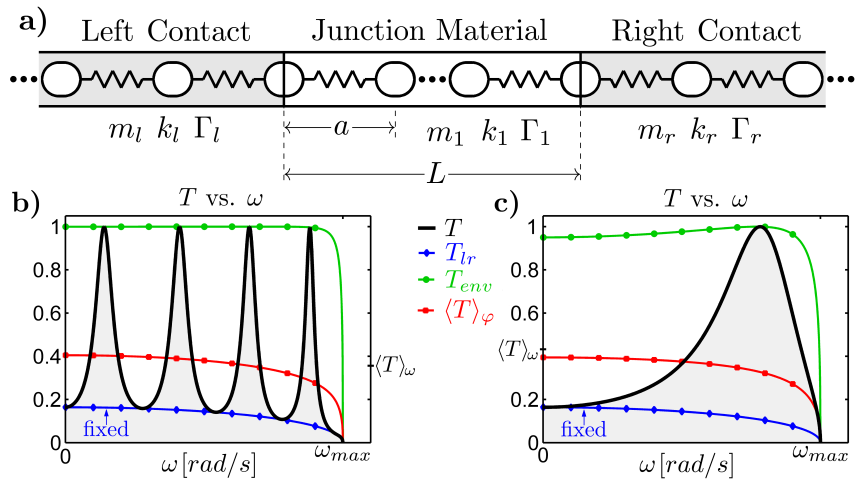

Figure 4.2: a) 1D crystal modeled as a chain of masses joined by springs. The masses at each contact-junction interface, balls with vertical lines, are chosen as the arithmetic mean of the neighboring masses to guarantee abrupt interfaces [6]. b) and c) The choice that maximizes $\langle T\rangle_{\omega}$ is an interplay between pushing the envelope up and fitting the right number of resonances inside the allowed frequency range. The figure shows an example where the largest envelope generated by the GM of contact broadenings (b) does not guarantee maximum $\langle T\rangle_{\omega}(\mathbf{c})$. It also shows the transmission for the abrupt interface between contacts $T_{l r}$ and the incoherent transmission $\langle T\rangle_{\varphi}$, which washes away interference (no wiggles). 
As shown in Fig. 4.2b, $T$ is an oscillating function bounded by the transmission of the abrupt interface between contacts

$$
T_{l r}=\frac{4 \gamma_{l r}}{\left(\gamma_{l r}+1\right)^{2}}
$$

which is prespecified, and an envelope function that depends on our choice of junction

$$
T_{e n v}=\frac{4 \gamma_{l r}}{\left(\gamma_{l 1}+\gamma_{1 r}\right)^{2}}
$$

Therefore, as long as $\Gamma_{1}$ lies in between $\Gamma_{l}$ and $\Gamma_{r}, T_{e n v}>T_{l r},\langle T\rangle_{\omega}>\left\langle T_{l r}\right\rangle_{\omega}$ and the ballistic thermal conductance of the gradual interface is larger than the abrupt interface.

Similar to an anti-reflection coating, we can eliminate reflection at a single frequency by destructive interference of the wave path undergoing a single reflection with the sum of paths undergoing more than one. The reflection coefficient $r$ is given by the sum of all the wave paths that return to the incident contact

$$
\begin{aligned}
r & =r_{l 1}+t_{l 1} r_{1 r} t_{1 l} e^{i 2 q_{1} L}+t_{l 1} r_{1 r}^{2} r_{1 l} t_{1 l} e^{i 4 q_{1} L}+\cdots \\
& =r_{l 1}+r_{1 r} \frac{t_{l 1} t_{1 l}}{1-r_{1 r} r_{1 l} e^{i 2 q_{1} L}}
\end{aligned}
$$

To eliminate reflection $R=|r|^{2}=0$, the factors in Eq. 4.7 must have equal magnitude and opposite phase. The first condition requires the junction broadening to be the GM of the contact broadenings $\Gamma_{1}=\sqrt{\Gamma_{l} \Gamma_{r}}$, and the second condition happens if the length of the junction $L_{1}$ fits exactly a quarter wavelength.

The choice that maximizes $\langle T\rangle_{\omega}$ is an interplay between pushing $T_{\text {env }}$ up and fitting the right number of resonances, since our choice of $\left(m_{1}, k_{1}, L_{1}\right)$ affects both the envelope function $T_{\text {env }}$ and the position of the resonances. Intuitively one might think that maximizing the envelope over the allowed frequency interval, by making $\Gamma_{1}$ the GM of the contact broadenings, generates the largest $\langle T\rangle_{\omega}$. However on a finite frequency range, the average fluctuates according to the number of resonances within the interval, with larger fluctuations for fewer resonances. For instance, the maximum $\langle T\rangle_{\omega}$ happens for a single resonance around $\omega_{\max } /\left(3 / 2-2 / 3 \pi^{2}\right)$ (Fig. 4.2c). This maximum is not very robust since it relies on our ability to place a single resonance at a particular frequency, which is limited by our experimental accuracy on $m_{1}, k_{1}$ and $L_{1}$.

A more robust condition consists of choosing $\Gamma_{1}$ as the GM of the contact broadenings over the allowed frequency interval. This condition maximizes the integral over $\omega$ of the phase $\left(\varphi=q_{1} L\right)$ average of the transmission $\langle T\rangle_{\varphi}$ (See Eq. 4.12)

$$
\Gamma_{G M}\left(m_{G M}, k_{G M}\right) \leftrightarrow \max _{\Gamma_{1}\left(m_{1}, k_{1}\right)} \int_{0}^{\omega_{\max }} d \omega \sqrt{T_{l r}} \sqrt{T_{e n v}}
$$


which is equivalent to pushing the envelope function up (Fig. 4.2b). Although choosing the GM condition does not guarantee the maximum $\langle T\rangle_{\omega}$, its difference with the maximum is bounded by $\langle T\rangle_{\text {max }}-\left\langle T_{G M}\right\rangle_{\omega} \lesssim$ $0.1\left(1-\left\langle T_{l r}\right\rangle_{\omega}\right)$ (This approximation follows from assuming a sinusoidal transmission oscillating between constant limits). But more importantly, the condition is more robust because it does not rely on interference effects.

The broadening for the GM condition $\Gamma_{G M}$ favors the flow of both low and high frequency phonons by making $\Gamma_{1}$ close to $\sqrt{\Gamma_{l} \Gamma_{r}}$ over the entire frequency range (Fig. 4.3b). Note that the GM of acoustic impedance by itself does not guarantee high $\langle T\rangle_{\omega}$ (Fig. 4.3a). Indeed, if our choice of $m_{1}, k_{1}$ makes $\omega_{c 1}<\omega_{\text {max }}$, phonon transmission is drastically cut by tunneling.
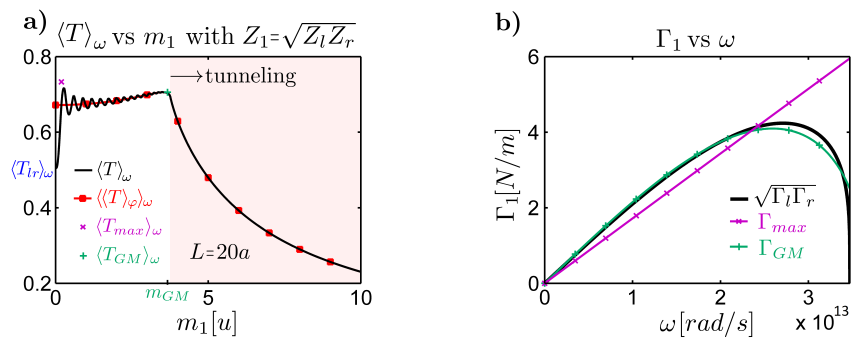

Figure 4.3: (a) Choosing the impedance $Z_{1}=\sqrt{Z_{l} Z_{r}}$ does not guarantee high $\langle T\rangle_{\omega}$ because if the cut-off frequency lies below $\omega_{\max }$, tunneling cuts down transmission (shaded region). On the other hand, choosing the broadening $\Gamma_{1} \approx \sqrt{\Gamma_{l} \Gamma_{r}}$ (b) fixes a unique pair $m_{G M}, k_{G M}$ that favors the flow of both low and high frequency phonons. The figure shows a similar result for the incoherent transmission average $\left\langle\langle T\rangle_{\varphi}\right\rangle_{\omega}$. Note that $\Gamma$ for the maximum $\langle T\rangle_{\omega}\left(\Gamma_{\max }\right)$ intersects $\sqrt{\Gamma_{l} \Gamma_{r}}$ where the single resonance is located (Fig. 4.2c).

A back of the envelope estimation for $m_{G M}$ and $k_{G M}$ follows by graphically overlapping $\Gamma_{1}\left(m_{1}, k_{1}\right)$ with $\sqrt{\Gamma_{l} \Gamma_{r}}$ (Fig. 4.3b). For low frequencies the slope of $\Gamma$ is dictated by the impedance, so to favor the flow of low frequency phonons we want $Z_{1}=\sqrt{Z_{l} Z_{r}}$. To favor the flow of high frequency phonons, we equate the frequency $\omega^{*}$ at which $\sqrt{\Gamma_{l} \Gamma_{r}}$ is maximum to the frequency at which $\Gamma$ is maximum $\omega_{c 1} / \sqrt{2}=\omega^{*}$. Then, from $Z=\sqrt{m k}$ and $\omega_{c}=2 \sqrt{k / m}$ we solve for $m_{G M}$ and $k_{G M}$

$$
k_{G M} \approx \frac{\omega_{c 1} Z_{1}}{2} \quad m_{G M} \approx \frac{2 Z_{1}}{\omega_{c 1}}
$$

From Eq. 4.9 we can also infer how to choose the junction sound velocity $v_{s}=a \sqrt{k / m}$ and the Debye temperature $T_{D}=\hbar \omega_{D} / k_{B}$ with $\omega_{D}=\omega_{c} \pi / 2$ and $k_{B}$ the Boltzmann constant. Using Eq. 4.3, we can also express the GM condition in terms of the density of states per unit cell (DOS)

$$
\Gamma=\frac{2 \omega m}{\pi D O S}
$$

which quantifies how to choose the overlap of density of states to maximize thermal conductance. 
When the junction material becomes a single mass or bond, the GM condition to maximize $\langle T\rangle_{\omega}$ transits to the AM of the contact masses or the HM of the contact spring constants respectively. This transition happens when $k_{1}$ increases enough or $m_{1}$ decreases enough to push $T_{\text {env }}$ below $T_{l r}$ (Fig. 4.2) making $\langle T\rangle_{\omega}\left\langle\left\langle T_{l r}\right\rangle_{\omega}\right.$. Then it is better to have an abrupt interface between the contacts which take us back to the AM or HM condition. In particular, if the spring constant $k_{1}>>\omega_{\max }^{2} m_{1}(L / a)^{2}$, then $\sin ^{2}\left(q_{1} L\right) \approx \omega^{2} m_{1} L^{2} / k_{1} a$ and $\Gamma_{1} \approx 2 \omega \sqrt{k_{1} m_{1}}$. Replacing the approximations into Eq. 4.2 and letting $k_{1}$ tend to infinity we recover the transmission for a single atomic junction [6]

$$
T \approx \frac{4 \gamma_{l r}}{\left(\gamma_{l r}+1\right)^{2}+\frac{4 \omega^{4} m_{1}^{2}(L / a)^{2}}{\Gamma_{r}^{2}}}
$$

The maximum of Eq. 4.11 happens when $m_{1}=0$, which recovers the AM condition for the atomic mass linking the contacts (Fig. 4.2a). Another way to rationalize the transition from the GM condition is by realizing that increasing $k_{1}$ or decreasing $m_{1}$ pushes the resonances out of the allowed frequency interval. Then, without resonances available to reach $T_{e n v}$, maximizing $T_{e n v}$ with the GM condition is no longer useful.

\subsection{Single material, Incoherent. Still Geometric Mean.}

When the phase gained by a phonon in its transit between interfaces is randomized, interference disappears and we can think of phonons as classical particles. The phase $\left(\varphi=q_{1} L\right)$ average of the transmission in Eq. 4.2 $\langle T\rangle_{\varphi}$ or the incoherent transmission is given by (Fig. 4.2)

$$
\langle T\rangle_{\varphi}=\frac{4 \gamma_{l r}}{\left(\gamma_{l r}+1\right)\left(\gamma_{l 1}+\gamma_{1 r}\right)}=\sqrt{T_{l r}} \sqrt{T_{e n v}}
$$

Since $T_{l r}$ is prespecified, this expression is once again maximized by the GM condition, for which $T_{\text {env }} \approx 1$ and $\left\langle T_{G M}\right\rangle_{\varphi} \approx \sqrt{T_{l r}}>T_{l r}$ (Fig. 4.2b). Note that Eq. 4.12 is the same expression obtained by adding incoherently all the possible wave paths from the left to the right contact

$$
\langle T\rangle_{\varphi}=T_{l 1} T_{1 r}+T_{l 1} R_{1 r} R_{1 l} T_{1 r}+\cdots=\frac{T_{l 1} T_{1 r}}{1-R_{1 l} R_{1 r}}
$$

with $T_{\alpha \beta}=4 \gamma_{\alpha \beta} /\left(\gamma_{\alpha \beta}+1\right)^{2}$, the transmission at the interface between material $\alpha$ and $\beta$, and $R_{\alpha \beta}=1-T_{\alpha \beta}$ the reflection.

The GM condition can be rationalized as a minimization of the sum of two opposite resistances, which are 
proportional to the ratio between phase average reflection and phase average transmission [52]. Reorganizing Eq. 4.13 to expose the additive property of resistance [53]

$$
\frac{\langle R\rangle_{\varphi}}{\langle T\rangle_{\varphi}}=\frac{1-\langle T\rangle_{\varphi}}{\langle T\rangle_{\varphi}}=\frac{1-T_{l 1}}{T_{l 1}}+\frac{1-T_{1 r}}{T_{1 r}}
$$

it follows that a variation of the junction produces opposite trends on the single interface resistances. Therefore, Eq. 4.14 is minimum when the individual resistances are equal, which leads to the GM condition.

Phase randomization can arise from lack of experimental control. For instance, the measured thermal conductance on a SAM junction is the sum of transmissions over molecules with slightly different lengths determined by the cross-sectional variation of the SAM. According to the variation strength, the average transmission can be approximated by

$$
\langle T\rangle_{\varphi_{0}-\delta}^{\varphi_{0}+\delta} \approx\left[T\left(\varphi_{0}\right)-\langle T\rangle_{\varphi}\right] \frac{\sin (2 \delta)}{2 \delta}+\langle T\rangle_{\varphi}
$$

with $2 \delta$ a uniformly distributed phase interval around $\varphi_{0}$ over which we average $T$. This expression follows from finding the effect of phase randomization on the peak of a sinusoidal function and using it to interpolate the two known limits. Note that $\delta=0$ recovers the fully coherent limit Eq. 4.2 while $\delta=\pi / 2$ the fully phase randomized limit Eq. 4.12.

In contrast to the coherent limit, the incoherent limit does not provide a condition for the junction length. To be consistent with our assumption that the phase is randomized between interfaces, we need to guarantee that $L_{1}$ is longer than the phonon phase coherent length. In case the phase randomization happens at the interfaces, $L_{1}$ is limited by the longest distance between interacting unit cells. That is the smallest block of material that allows us to define impedance.

\subsection{N Materials, Coherent. Exponential.}

For several junction materials in between the contacts (Fig. 4.4a), enforcing the GM condition for every three consecutive pieces translates into an exponentially varying broadening (Eq. 4.18). This trend follows from taylor expanding $\Gamma(x)=\sqrt{\Gamma(x+\Delta x) \Gamma(x-\Delta x)}$ to second order and solving the corresponding differential equation $\left(\Gamma^{\prime}\right)^{2}=\Gamma \Gamma^{\prime \prime}$. As we increase the number of materials, this variation generates a banded transmission that monotonically widens. In this way, we approach the maximum limit of thermal conductance imposed by the contacts. The idea was inspired by the exponentially tapered impedance coupling used to design 
broad band transmission lines [72], which constitutes just one example of the extrapolation possibilities from Microwave engineering to phonon engineering.

The transmission for a system with $N$ junction materials is a complicated function dictated by interference patterns. However, to maximize transmission, we intuitively expect a small reflection at each interface so we can approximate the reflection coefficient $r$ by the sum of wave paths undergoing a single reflection, i.e.

$$
r \approx r_{l 1}+r_{12} e^{i 2 \varphi_{1}}+r_{23} e^{i 2 \varphi_{2}}+\cdots+r_{N r} e^{i 2 \varphi_{N}}
$$

At this point we have $N$ exponential basis vectors to fit a desired reflection function, which is a rich problem with different answers according to the design criteria for the function. We further simplify the expression by using our intuition of anti-reflection coating, where to eliminate the first reflection $r_{l 1}$ we need $r-r_{l 1}$ to have the same magnitude and opposite phase. Then we assume equal phase gained between interfaces, i.e. $\varphi_{n}=2 n \varphi$, to get complex roots of 1 equally spaced in Eq. 4.16, and equal reflection at each interface $r_{i, i+1}=r_{i+1, i+2}=r_{i}$ to assure equal magnitude. Then Eq. 4.16 turns to

$$
r \approx r_{i} \sum_{j=0}^{N} e^{i 2 j \varphi}=r_{i} \frac{1-e^{i 2(N+1) \varphi}}{1-e^{i 2 \varphi}}
$$

As $N$ increases, the resonances $\left(r=0 \leftrightarrow e^{i 2(N+1) \varphi}=1\right)$, the anti-resonances $\left(e^{i 2(N+1) \varphi}=-1\right)$ and the decreasing trend of $r_{i}$ generate a reflection that broadens and tends to zero or equivalently a transmission that broadens and tends to unity. Fig. $4.4 \mathrm{~b}$ shows the widening of the transmission when we increase the number of junctions from $N=1$ to $N=5$.

Equal reflection at each interface follows from imposing the GM condition on every three consecutive materials, which leads to an exponential variation

$$
\Gamma_{n}=\Gamma_{l}^{\frac{N+1-n}{N+1}} \Gamma_{r}^{\frac{n}{N+1}}=\Gamma_{l} e^{\zeta n}
$$

with $\zeta=\ln \left(\Gamma_{l} / \Gamma_{r}\right) /(N+1)$. This variation maximizes the frequency average of the incoherent transmission (See Eq. 4.22 and below). Therefore we find the $m_{n}$ and $k_{n}$ that better satisfy Eq. 4.18 by maximizing $\left\langle\langle T\rangle_{\varphi}\right\rangle_{w}$. Similar to Sec. 4.2, a useful back of the envelope approximation follows by graphically overlapping the desired and possible broadenings. To match the low frequency slope we need $Z_{n}=Z_{l}^{N+1-n / N+1} Z_{r}^{n / N+1}$ and to match the high frequency spectrum we need $\omega_{c n}=\sqrt{2} \omega_{n}^{*}$, with $\omega_{n}^{*}$ the frequency at the maximum 

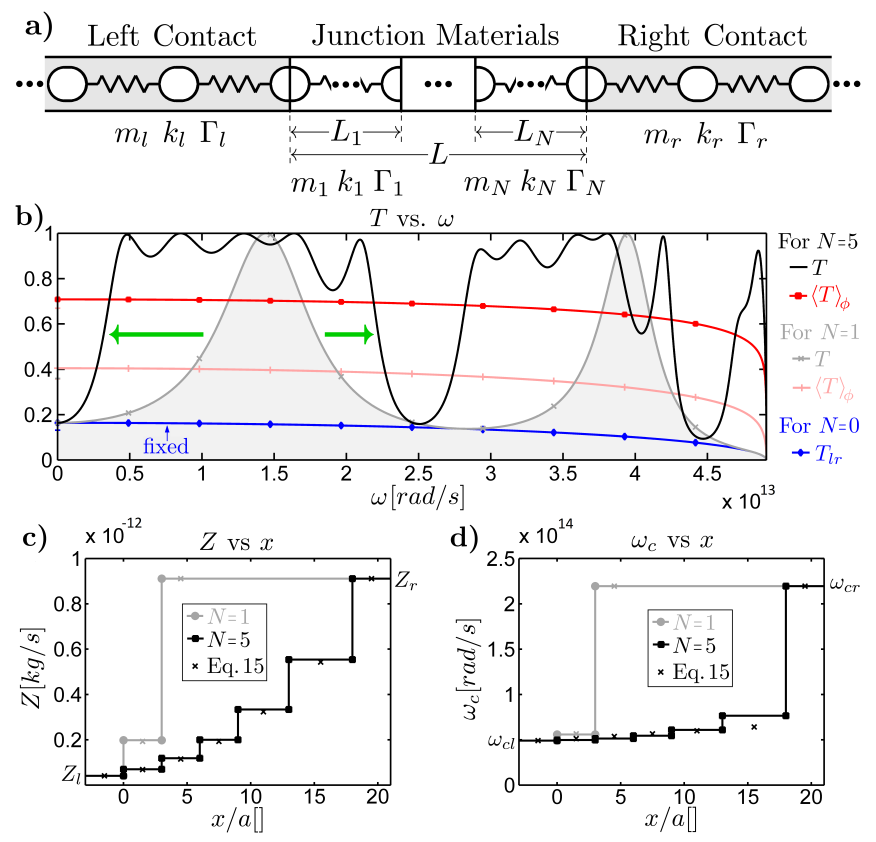

Figure 4.4: a) 1D crystal modeled as a chain of masses joined by springs. b) Transmission $T$ and its phase average $\langle T\rangle_{\varphi}$ for contacts coupled through an exponential variation of broadenings with a single junction $N=1$ and with $N=5$ junction materials. As we increase the number of materials transmission widens increasing $\langle T\rangle_{\omega}$. The spatial variation of acoustic impedance is shown in c) and of cut-off frequency in d) for $N=1$ and $N=5$. The crosses show the back of the envelope calculation given by Eq. 4.19.

value of Eq. 4.18. Then $m_{n}$ and $k_{n}$ are approximated by

$$
k_{n} \approx \frac{\omega_{c n} Z_{n}}{2} \quad m_{n} \approx \frac{2 Z_{n}}{\omega_{c n}}
$$

and rules for the variation of the sound velocity, Debye temperature and overlap of density of states can be found following Sec. 4.2. Fig. 4.4c,d show the spatial variation of junction impedances and cut-off frequencies for $N=1$ and $N=5$. Note that the trend of impedance follows an exponential variation that favors flow of low frequency phonons while the trend of cut-off frequencies is stable around $\omega_{\text {max }}$ to favor flow of high frequency phonons.

Equal phase gain between consecutive interfaces needs

$$
q_{n} L_{n}=\frac{L_{n}}{a} 2 \sin ^{-1}\left(\frac{\omega}{\omega_{c n}}\right)=\varphi
$$

which we can only satisfy at a single frequency $\omega^{* *}$. A careless choice of $\omega^{* *}$ and $\varphi$ might lead to impractically small lengths $\left(L_{n} \sim 2 a\right)$ or long lengths where phonons are not coherent $\left(L_{n} \sim\right.$ mean free path). A sensible choice is for example $\omega^{* *}=\omega_{\max } / 2$ and $\varphi=2 \pi$, which generates lengths on the order of $L_{n} \sim 10 a \sim 5 \mathrm{~nm}$.

The theory of small reflections shown in this section is valid as long as we can neglect the contributions 
from paths that involve more that one reflection, i.e. $\left|r_{i}\right|^{3}<<\left|r_{i}\right|$. For $\Gamma_{l}<\Gamma_{r}$ and if we consider $\left|r_{i}\right|<0.1$ small enough, the minimum number of junctions $N_{\min }$ needed to be in this regime is

$$
N_{\text {min }} \approx 1.5 \ln \left(\frac{\Gamma_{r}}{\Gamma_{l}}\right)-1
$$

For instance, for a 10-fold mismatch $\Gamma_{r} / \Gamma_{l}=10 N_{\min } \approx 2.5$ and for a 100 -fold mismatch $N_{\min } \approx 6$.

\subsection{N Materials, Incoherent. Back to Exponential.}

When phonon phase is randomized in between interfaces, the exponentially varying broadening minimizes resistance and generates maximum $\left\langle\langle T\rangle_{\varphi}\right\rangle_{\omega}$. Total resistance decreases as the number of junction materials $N$ increases because the addition of resistance coming from new interfaces is dominated by the decrease on interfacial resistance due to less mismatch. Nevertheless, as we increase $N$, the length between contacts increases and other scattering mechanism become important. Therefore, there is a sweet spot for $N$ at which we stop increasing thermal conductance by adding junction materials.

Transmission when phonon phase is randomized or incoherent transmission $\langle T\rangle_{\varphi}$ can be found from the total resistance of the system similar to Eq. $4.14[52,53,24]$

$$
\frac{1-\langle T\rangle_{\varphi}}{\langle T\rangle_{\varphi}}=\sum_{j} \frac{1-T_{j}}{T_{j}} .
$$

Eq. 4.22 becomes minimum when all the factors on the right hand side are equal. This condition needs the ratios of broadenings between consecutive materials to be the same and leads to the exponential variation in Eq. 4.18 (Fig. 4.4b). Replacing that variation into Eq. 4.22 give us the maximum $\langle T\rangle_{\varphi}$, which can be approximated by

$$
\langle T\rangle_{\varphi} \approx \frac{4 N}{\ln ^{2}\left(\Gamma_{l} / \Gamma_{r}\right)+4 N}
$$

when $N$ tends to infinite and by

$$
\langle T\rangle_{\varphi} \approx\left(T_{l r}\right)^{(1 / N+1)}
$$

for small $N$. As shown in Fig. 4.5a, $\langle T\rangle_{\varphi}$ increases as $N$ increases. This non intuitive result happens because the increment in resistance by the addition of an interface is dominated by the decrease of the interfacial resistance of all the interfaces due to less material mismatch. 

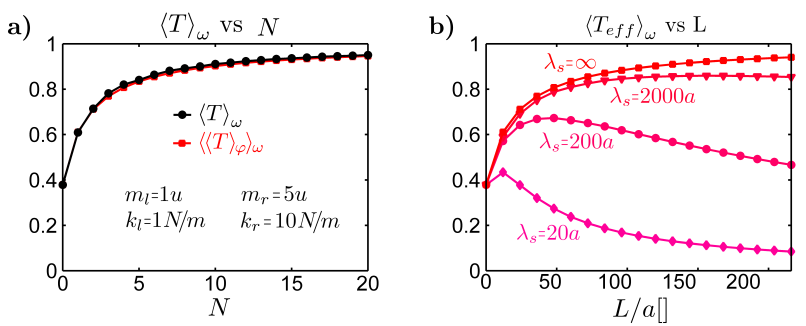

Figure 4.5: a) Using the exponential variation of broadenings, as the number of junction materials $N$ increases, $\langle T\rangle_{\omega}$ and $\left\langle\langle T\rangle_{\varphi}\right\rangle_{\omega}$ approach to one. b) As $\mathrm{N}$ increases the length between contacts increases and phonon flow is affected by scattering mechanism different from interfacial scattering. Therefore, there is a sweet spot for $N$ at which we stop increasing thermal conductance by adding junction materials.

As the number of junction materials increases, the length between contacts also increases and other scattering mechanisms besides interfacial scattering become important. We can combine all of them using Matthiessen's rule to obtain an effective mean free path $(\mathrm{mfp})$ in terms of the mfp for interfaces $\lambda_{i}$ and the mfp for other scattering mechanisms $\lambda_{s}$

$$
\frac{1}{\lambda_{e f f}}=\frac{1}{\lambda_{i}}+\frac{1}{\lambda_{s}}
$$

and an effective transmission

$$
\langle T\rangle_{\varphi}^{e f f}=\frac{\lambda_{e f f}}{L+\lambda_{e f f}}
$$

Note that Eq. 4.25 and 4.26 can be derived from equation $4.22[53,24]$. We define the interfacial mfp for $N$ materials following the exponential variation $\lambda_{i}$ by replacing Eq. 4.18 into Eq. 4.22 , defining the distance between interfaces as $L_{i}=L / N$ and comparing with Eq. 4.26 so that

$$
\langle T\rangle_{\varphi}=\frac{\lambda_{i}}{L+\lambda_{i}} \quad \text { and } \quad \lambda_{i}=\left(\frac{L L_{i}}{L+L_{i}}\right) \frac{T_{i}}{R_{i}}
$$

$\lambda_{i}$ is dominated by $L_{i}$ since we expect $L_{i}<L$ and is inversely proportional to the resistance at each interface. Thus, as $N$ increases $\lambda_{i}$ also increases. However, the effective mfp is bounded by $\lambda_{s}$. Thus, there is a sweet spot for the number of junction materials at which adding more interfaces does not increase the effective mfp and $\langle T\rangle_{\varphi}^{e f f}$ is dominated by the increment of $L$ (Fig. 4.5b).

\subsection{Summary of Contributions}

1. We show that to maximize thermal conductance, in $1 \mathrm{D}$ single junction between prespecified contacts systems within the coherent and incoherent regimes, we should choose the properties of the junction so its broadening is as close as possible to the GM of the contact broadenings. This requires two conditions: (1) the impedance equal to the GM of the contact impedances to favor low frequency phonons and (2) 
the cut-off frequency close to the minimum of the contact cut-off frequencies to favor high frequency phonons.

2. We translate those conditions into rules to choose the junction mass, spring constant, sound velocity, Debye temperature or density of states. These rules allow us to pinpoint the role of each quantity in bridging phonon across the interface.

3. We show that to maximize thermal conductance in the multi-junctions case, the GM condition evolves into an exponential variation of broadenings. This variation pushes thermal conductance to the maximum limit allowed by the contacts as the number of materials increases.

4. We integrate interfacial scattering with other scattering mechanisms and find a sweet spot for the number of material caused by the interplay between smoothing the interface by impedance matching and enlarging the distance between contacts. 


\section{Chapter 5}

\section{Conductance Across One Boundary in}

\section{$3 \mathrm{D}$}

The addition of random atoms at an abrupt interface (Fig. 5.1a and b) generates two effects in the harmonic regime: 1) it decreases phonon transmission of the transport channels that conserve transverse momentum because it adds alloy backscattering $[73,74,75,76]$; and, 2) it opens up new channels that do not conserve transverse momentum because it breaks the translational symmetry at the interfacial plane [77].

In this chapter, we demonstrate that the relative conductance between interfaces with different morphologies (Fig. 5.1) depends on crystal structure. For the systems considered in this study, adding a unit cell monolayer of mixing to an abrupt interface always enhances the interfacial conductance, but the extent varies over an order of magnitude according to the crystal structure (Fig. 5.2). Furthermore, the conductance across a mixed interface does not always increase relative to that at a uniform interface. In fact, while the conductance increases for simple cubic (SC) and diamond cubic (DC) crystal structures, it decreases for face centered cubic (FCC) crystals. This suggests that the commonly invoked virtual crystal approximation, which models the mixed interface as a uniform interface, alternatively overestimates or underestimates the effect of interfacial mixing on thermal conductance. For DC crystalline interfacial regions, we show that the enhancement of conductance by mixing depends on phonon polarization. For instance, mixing increases transmission between TA-TA modes but not between LA-LA modes.

We explain our results within the Landauer theory, where thermal conductance is directly related to the product of the number of conducting channels or modes $(M)$ times their average transmission $(T)$. We find that 1) the crystal structure determines the relative magnitude of the minimum of the contacts' modes $M_{\min }$ vs. the conserving modes $M_{c}$ that conserve the component of phonon momentum transverse to the 
a) Abrupt

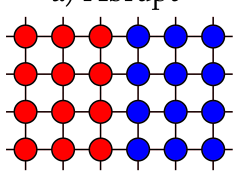

b) Mixing

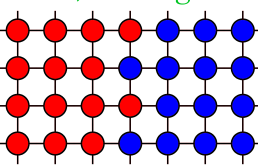

c) Uniform

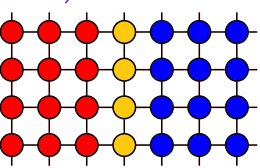

Figure 5.1: a) Abrupt interface. b) Interface with random atomic mixing (mixed interface). c) Interface with an added homogeneous layer at the junction (uniform interface).

interface. On the other hand, 2) the interface morphology determines if phonons move through $M_{\min }$ for mixed interfaces, or $M_{c}$ otherwise. Based on these two concepts, we show that the conductance across a mixed interface increases relative to that at a uniform interface when $M_{\min }>M_{c}$, with larger degree of enhancement as the inequality increases. The larger enhancements, seen in SC and in TA branches of DC, are associated with the emergence of voids in the conserving modes $\left(M_{c}=0\right)$. Such voids in turn arise when the subbands shift but do not distort with increasing momenta.

We start by deriving an inequality (Eq. 5.4) between the conductance of the mixed and uniform interfaces from the Landauer theory (Sec. 5.1). Then, we describe how the modes (Sec. 5.2) and transmission (Sec. 5.3) shape that inequality according to phonon polarization. In Sec. 5.3, we derive analytical expressions for the transmissions of the scalar SC and FCC systems. For the uniform interface we find a maximum conductance when the junction mass is the arithmetic mean of the contact masses. For the mixed interface, we find that the transmission between phonons that do not conserve transverse wavevector, $k_{\perp}$, depends on the difference of the contact masses and on the alloy scattering factor, $\alpha(1-\alpha)$ with $\alpha$ the fraction of heavy atoms at the interface.

\section{$5.1 \quad$ Methodology}

We start from the equation defining the thermal conductance Eq. 2.1 and the fact that the product $M T$ equals the sum of the phonon transmissions between modes on the left and right contacts.

For the uniform interface (Fig. 5.1c), the symmetry in the transverse direction requires that phonons crossing it conserve their transverse wavevector $k_{\perp}$. Thus, the nonzero contributions to $M T$ are transmissions $T_{k_{\perp}, k_{\perp}}$ between contact modes with the same $k_{\perp}$. Referring to the number of these transmissions as $M_{c}$, the conserving modes, and their average as $T_{c}$, we can express $M T$ for the uniform interface as

$$
M T_{u n i}=\sum_{k_{\perp}} T_{k_{\perp}, k_{\perp}}=M_{c} T_{c}<M_{c}
$$

$M_{c}$ is given by the overlap between the projections of the frequency isosurfaces of the contacts onto the $k_{\perp}$ 
plane (Fig 5.3b). Note its role as an upper bound of $M T_{\text {uni }}$. Also note that the abrupt interface (Fig. 5.1a) is a limiting case of the uniform interface.

When we replace the homogeneous interfacial layer of the uniform interface by random contact atoms (Fig. 5.1b), the atomic disorder breaks the transverse symmetry and allows phonon transmission $T_{k_{\perp}, k_{\perp}^{\prime}}$ between modes that do not conserve $k_{\perp}$ [77]. That disorder also decreases the transmission $\left(\delta T_{c \downarrow}\right)$ between modes that conserve $k_{\perp}$. We can express MT for the mixed interface as

$$
\begin{aligned}
M T_{\text {mix }} & =\sum_{k_{\perp}} T_{k_{\perp}, k_{\perp}}+\sum_{k_{\perp} \neq k_{\perp}^{\prime}} T_{k_{\perp}, k_{\perp}^{\prime}} \\
& =\underbrace{M_{c}\left(T_{c}-\delta T_{c \downarrow}\right)}_{\text {conserving }}+\underbrace{M_{n c} \delta T_{n c \uparrow}}_{\text {non-conserving }}<M_{\min },
\end{aligned}
$$

where $M_{n c} \delta T_{n c \uparrow}$ represents the increase in conductance due to the newly available channels. We define $M_{n c}$ as the minimum of the contacts' modes, $M_{\min }=\min \left(M_{l}, M_{r}\right)$, because $M T_{m i x}$ is bounded by $M_{\min }$. This result follows from the conservation of energy current. $M T \leq M_{l}$ because the transmission from each mode on the left contact is less or equal than one. Reversing the argument, we also conclude that $M T \leq M_{r}$.

Comparing Eq. 5.1 and 5.3, the conductance of the mixed interface is larger than that of the uniform interface, $G_{m i x}>G_{u n i}$, if

$$
\int_{0}^{\infty} \frac{d \omega}{2 \pi} \hbar \omega \frac{\partial N}{\partial \mathbf{T}} M_{m i n} \delta T_{n c \uparrow}>\int_{0}^{\infty} \frac{d \omega}{2 \pi} \hbar \omega \frac{\partial N}{\partial \mathbf{T}} M_{c} \delta T_{c \downarrow}
$$

In other words, $G_{m i x}>G_{u n i}$ if the gain in conductance by opening new channels that do not conserve $k_{\perp}$ $\left(M_{n c} \delta T_{n c \uparrow}\right)$ surpasses the loss in conductance by phonons conserving $k_{\perp}\left(M_{c} \delta T_{c \downarrow}\right)$ over a window set by the cut-off frequency and the temperature.

\subsection{Minimum vs. Conserving Modes}

We calculate the harmonic conductance of abrupt, uniform and mixed interfaces embedded into four different crystal structures: 1) SC and 2) FCC crystals, where the atomic movements are simplified to a single direction and thus the interatomic force constants (IFCs) are scalars; 3) FCC crystal with IFCs calculated from the Lennard-Jones (LJ) potential; and 4) DC crystal with IFCs calculated from density functional theory (DFT). The interfacial region for each system consists of a monolayer of primitive unit cells (Fig. 1). The same IFCs and lattice constants are used throughout each system to simplify the analysis. When mass variation is much larger than IFCs variation, the effect of mass disorder dominates the scattering of cross-species interactions 
[78]. However, as the inequality decreases, the effect of IFC variation will also play an important role [79]. The ratio between the atomic masses of the contacts is 3 for the SC and FCC systems and 2.6 for the DC system, corresponding to the mass ratio of $\mathrm{Si}$ and Ge. The conductance is calculated using NEGF, and the details of the simulations and assumptions are given in Sec. 5.4.
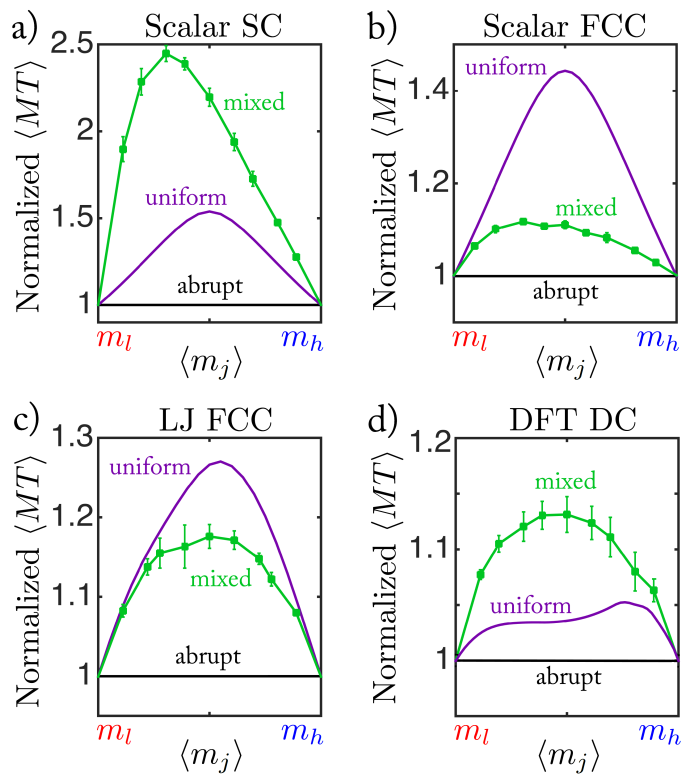

Figure 5.2: Plot of the average $M T$ normalized by the results for an abrupt interface versus the average mass at the junction layer for the cases described at the beginning of the section. Adding mixing at an abrupt interface enhances the interfacial conductance in all systems simulated in this work, but the extent depends on crystal structure. Compared to uniform interfaces, however, mixing does not always yield an increase in conductance.

Figure 5.2 plots the frequency average of $M T$, which one can interpret as conductance without the "low" temperature dependence (Eq. 2.1), vs. the average mass at the interfacial layer $\left\langle m_{j}\right\rangle$ for the different crystal structures. For these systems, adding a unit cell monolayer of mixing to the abrupt interface always enhances the interfacial conductance, but the extent depends on crystal structure. For the scalar SC crystal (Fig. 5.2a), the maximum relative change of $\langle M T\rangle$ between the mixed and abrupt interfaces is about $((2.5-1) / 1 \times 100 \%)=150 \%$. However, for the FCC and DC crystals (Fig. 5.2b, c and d), the relative change of $\langle M T\rangle$ is only about $13 \%$. Furthermore, the conductance across a mixed interface does not always increase relative to that at a uniform interface. In fact, it increases for SC and DC crystals but decreases for FCC crystals.

The increment of conductance from the abrupt interface to the mixed interface (Fig. 5.2) relies on the atomic extent of the mixing region. For this special case, Eq. 5.4 tells us that the gain in conductance by phonons that do not conserve $k_{\perp}$ surpasses the loss in conductance by phonons conserving $k_{\perp}\left(M_{n c} \delta T_{n c \uparrow}>M_{c} \delta T_{c \downarrow}\right)$. As the extent of the mixing region increases, phonon back scattering increases and transmission decreases. 
Thus, $\delta T_{n c \uparrow}$ decreases while $\delta T_{c \downarrow}$ increases, making the inequality more difficult to be satisfied. At some point, the inequality stops being true and $G_{m i x}$ becomes less than $G_{a b r}$, which is the usual experimental outcome $[2]$.

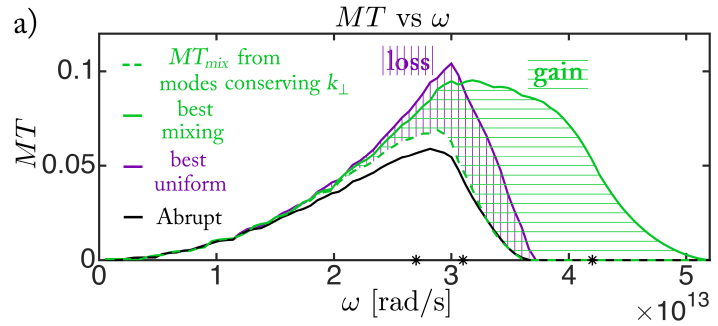

b) Available modes in the contacts

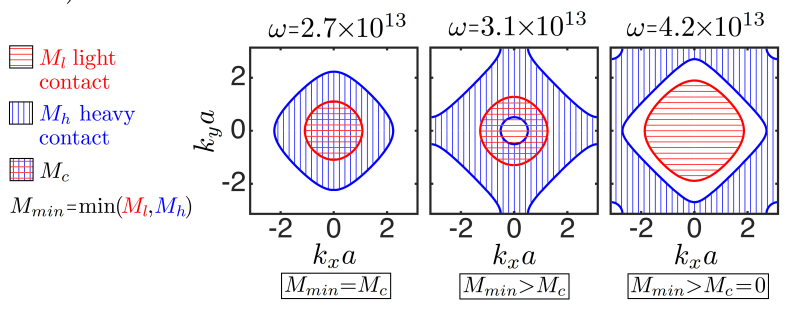

Figure 5.3: a) For SC, the conductance for the mixed interface is larger than the uniform interface because the loss in transmission, $T$, due to the extra scattering brought by the random atoms (area with vertical lines) is dominated by the gain in modes, $M$, coming from transmissions between phonon that do not conserve $k_{\perp}$ (area with horizontal lines). b) While the $M T_{u n i}$ spectrum is limited by transmissions between phonon conserving $k_{\perp}$ (overlap region), the extra $M T_{m i x}$ spectrum comes from transmissions between phonons that do not conserve $k_{\perp}$.

For the SC crystal, the large conductance increase of the mixed interface results from the wider $M T$ spectrum (Fig. 5.3a). This extra spectrum comes only from transmissions between modes that do not conserve $k_{\perp}$. In fact, over that frequency interval, the available contact modes do not overlap (Fig. 5.3b), banning transmissions between modes conserving $k_{\perp}$. Thus, $M_{c}=0$ and $M T_{u n i}=0$. Mixing removes the requirement of conserving $k_{\perp}$, opening $M_{\text {min }}$ conduction channels and making $M T_{\text {mix }}>0$.

Figure 5.3a shows $M T$ for the mixed, uniform and abrupt interfaces. $M T_{m i x}$ is split into the contributions from modes conserving $k_{\perp}$ and those that do not. This gives us a pictorial representation of Eq. 5.4: $G_{m i x}>G_{u n i}$ because the $M T$ area gained due to transmissions between modes not conserving $k_{\perp}$ is larger than the $M T$ area lost due to disorder among the modes that conserve $k_{\perp}$.

A similar pictorial representation for the scalar FCC interfaces is shown in Fig. 5.4a. In this case, $G_{u n i}>G_{m i x}$ because the $M T$ area gained due to transmissions between modes not conserving $k_{\perp}$ is less than the $M T$ area lost due to disorder among the modes that conserve $k_{\perp}$. Note that $M T_{u n i}$ and $M T_{m i x}$ cover the same frequency range, and the overlap of the contacts' modes $M_{c}$ equals their minimum $M_{\text {min }}$ over most of the spectrum (Fig. 5.4b). Thus, the accessible modes on the mixed interface $M_{\min }$ do not bring any advantage over the existing modes $M_{c}$ on the uniform interface (Eq. 5.4). The dominant conductance is then 


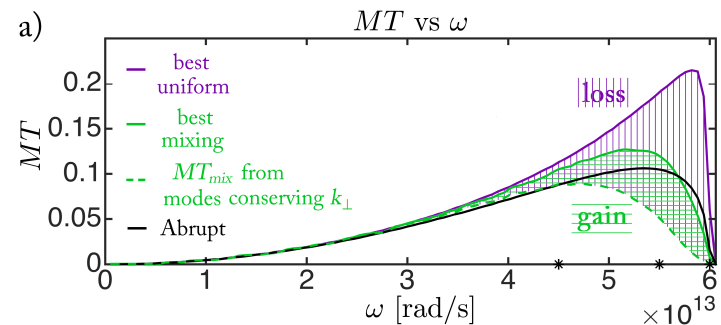

b) Available modes in the contacts

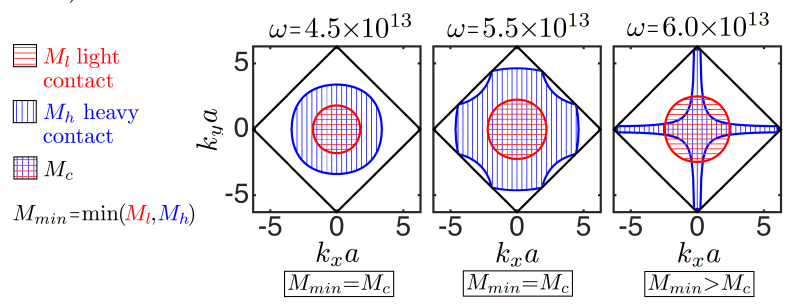

Figure 5.4: a) For FCC, the conductance for the uniform interface is larger than the mixed interface because the loss in $T$ due to the extra scattering brought by the random atoms (area with vertical lines) dominates the gain in $M$ coming from transmissions between phonon that do not conserve perpendicular momentum (area with horizontal lines). b) $M_{c} \approx M_{\min }$ over most of the spectrum. Thus, mixing provides little advantage by allowing transmission between modes not conserving $k_{\perp}$.

decided by the transmission, which in this case favors the loss in the conserving modes over the gain in the non-conserving ones.

From Fig. 5.3 and 5.4, we note that the relative magnitude between $M_{m i n}$ and $M_{c}$ plays an important role determining the larger $M T$ between the mixed and uniform interfaces (Eq. 5.4). This is not surprising because of their roles as $M T$ upper bounds for the mixed and uniform cases respectively. We can distinguish three cases: when 1) $M_{c} \approx M_{\text {min }}$, the modes conserving $k_{\perp}$ reach the physical limit of modes that can carry heat in one of the contacts. Equation 5.4 tells us that the transmission alone decides the dominant $M T$, which can be either the uniform or mixed $M T$. For the scalar SC and FCC structures, whenever $M_{c} \approx M_{m i n}$ we see that $M T_{u n i}>M T_{m i x}$ (Fig. 5.3 and 5.4). Therefore the loss in transmission on the conserving modes surpasses the gain in transmission on the non-conserving modes $\left(\delta T_{c \downarrow}>\delta T_{n c \uparrow}\right.$ in Eq. 5.4). When 2) $M_{\text {min }}>M_{c}$, the dominant $M T$ results from a balance between the added modes that do not conserve $k_{\perp}$ and the loss in transmission on the modes that conserve $k_{\perp}$ (Eq. 5.4). For instance, in the $\mathrm{SC}$ structure, $M T_{m i x}$ becomes larger than $M T_{u n i}$ as the ratio $M_{m i n} / M_{c}$ increases (Fig. 5.3). When 3) $M_{m i n}>M_{c}=0$, $M T_{\text {mix }}>M T_{u n i}=0$ and $M T_{\text {mix }}$ is only due to transmissions between modes that do not conserve $k_{\perp}$ as shown by Fig. 5.3. These three criteria may help in the search for interfacial materials where a particular outcome is expected from atomic mixing over the harmonic regime.

For the diamond crystal, the polarization of the incident and transmitted phonons plays an important role in deciding the outcome of the dominant conductance. We give a brief description of the calculation process in Sec. 5.5. Figure 5.5a shows that $G_{m i x}>G_{u n i}$ mostly because of TA phonons in the light contact transitioning 
to TA and LA phonons in the heavy material. The transmissions between phonons that do not change polarization behave similarly to the scalar crystals. For TA-TA and LA-LA transmissions, $M T_{m i x}>M T_{u n i}$ when $M_{\text {min }}>M_{c}$ and $M T_{u n i}>M T_{\text {mix }}$ when $M_{\min } \approx M_{c}$ (Fig. 5.5b). The transmission between other polarizations will be analyzed in the next section.
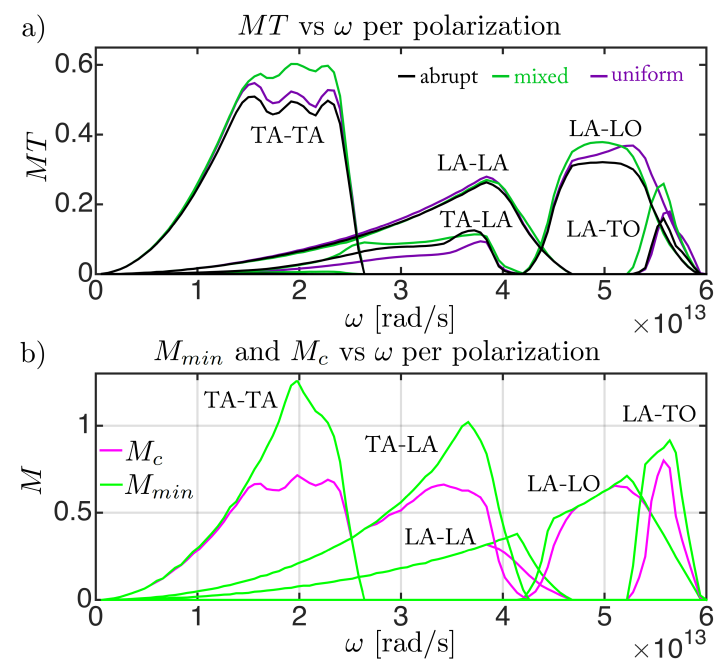

Figure 5.5: a) MT for DC crystal split in polarizations. The conductance of the mixed interface dominates mostly because mixing favors transmissions between TA-TA and TA-LA modes, where the ratio of $M_{m i n} / M_{c}$ is larger. b) $M_{\min }$ and $M_{c}$ for the different polarization branches.

The polarized modes for the diamond crystal uncover an interesting similarity between the modes of the SC and the TA branches in DC and between the modes of the scalar FCC and the LA branch in DC (Fig. 5.6). For the cases where mixing significantly enhances conductance (SC and TA-TA branches in DC), we see a common central void in the modes for the heavy contact. This void arises in phonon bands where the $k_{\perp} \approx 0$ subbands only cover a fraction of the whole band spectrum (Fig. 5.7). Indeed, after the cutoff frequency of those subbands, the $k_{\perp} \approx 0$ modes, or central modes, start to become unavailable. From another point of view, the void originates when the upward shift of the $k_{\perp} \approx 0$ subbands dominate their shrinking as $\left|k_{\perp}\right|$ increases. We see this happening for SC and TA-TA but not for FCC and LA-LA (Fig. 5.7).

For the scalar SC and FCC crystals, the existence of the void can be associated with the independence of interlayer coupling as $k_{\perp}$ increases. For a SC crystal with atomic mass $m$ and interatomic force constant $f$, the subbands are given by

$$
\omega^{2} m=f_{\text {on }}-2 f_{\text {off }} \cos \left(k_{z} a\right)
$$

with the onsite coupling $f_{\text {on }}=6 f-2 f \cos \left(k_{x} a\right)-2 f \cos \left(k_{y} a\right)$ representing the atomic interactions within a transverse layer of atoms, and the offsite coupling $f_{\text {off }}=f$ representing the interaction between layers. As the magnitude of $k_{\perp}$ increases $f_{\text {on }}$ increases, shifting upwards the subband but $f_{\text {off }}$ remains constant 
a) Available TA modes for $\mathrm{Si}$ and $\mathrm{Ge}$

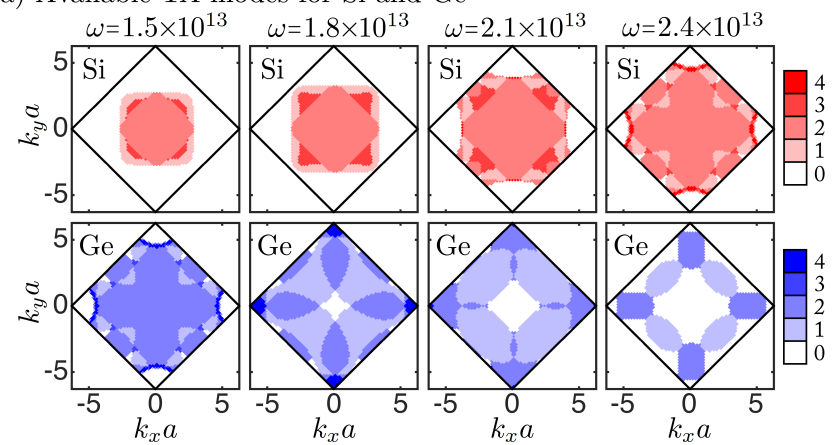

b) Available LA modes for $\mathrm{Si}$ and Ge

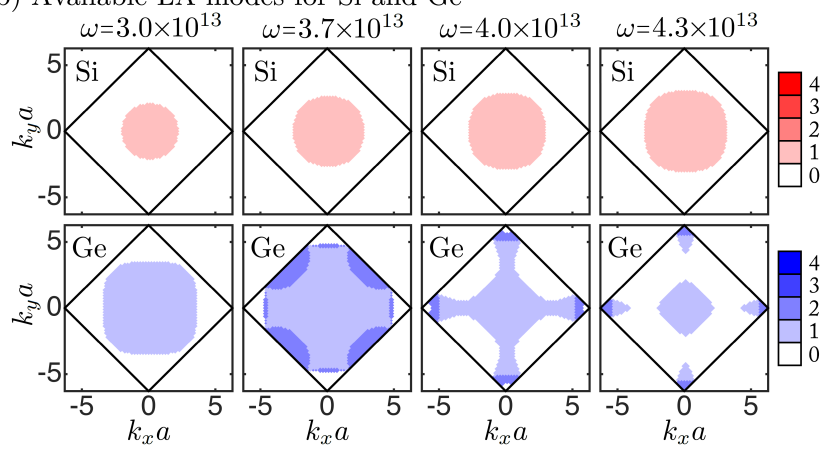

Figure 5.6: Available modes in the DC contacts for the (a) TA and (b) LA branches. The modes for SC crystal and the TA branches, where mixing enhances the most the conductance of an abrupt interface, present a central void that enhances the ratio $M_{\min } / M_{c}$.

keeping their width stable. On the other hand, for a FCC crystal the subbands are given by

$$
\omega^{2} m=f_{\text {on }}-2 f_{\text {off }} \cos \left(k_{z} \frac{a}{2}\right)
$$

with $f_{\text {on }}=12 f-4 f\left[\cos \left(k_{x} a / 2\right) \cos \left(k_{y} a / 2\right)\right]$ and $f_{\text {off }}=2 f\left[\cos \left(k_{x} a / 2\right)+\cos \left(k_{y} a / 2\right)\right]$. As the magnitude of $k_{\perp}$ increases, $f_{\text {on }}$ increases shifting the subbands upward, but at the same time, $f_{\text {off }}$ decreases shrinking their bandwidth.
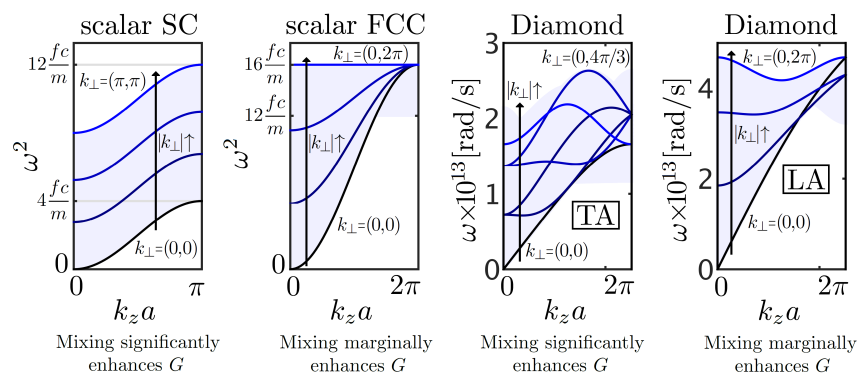

Figure 5.7: When the shift in the subbands dominate their distortion, the $k_{\perp}=0$ subband only covers a fraction of the whole band spectrum. This generates a void in the conserving modes, which is seen when mixing significantly enhances the conductance of an abrupt interface. The solid lines represent subbands for different $k_{\perp}$ and the shaded region is the projected dispersion on $k_{z} a$. 
Although we neglect anharmonicity in this paper, we hypothesize that our main conclusions should hold even when anharmonicity is present. Phonon-phonon interaction enables inelastic transmission of phonons at the interface. However, the transverse symmetry selection rules for $k_{\perp}$ continue to hold. Therefore, phonons crossing an abrupt or uniform interface have to conserve $k_{\perp}$ and are restricted to inelastic jumps within the conserving modes $M_{c}$. On the other hand, phonons crossing a mixed interface can jump in frequency within the minimum of the contacts' modes $M_{\min }$. Thus, we expect a similar relation between the crystal structure, which determines the ratio $M_{m i n} / M_{c}$, and the relative magnitude of the conductance for the abrupt, uniform and mixed interfaces. Further studies are required to evaluate the validity of this hypothesis. We also expect a conductance increase for all the systems considered in this work, since anharmonicity allows transmission of phonons with frequencies beyond the elastic limit. Thus as temperature and thereby anharmonicity increases, interfacial thermal conductance increases [80, 81]. For some systems with large Debye temperatures anharmonicity can be neglected. For instance, Si/Ge interfaces present a thermal conductance relatively independent of temperature below $500 \mathrm{~K}$, which indicates that phonon-phonon interactions are not dominant over that temperature range [81].

\subsection{Gain vs. loss in Transmission}

An essential part of the conductance inequality (Eq. 5.4) is the transmission, which can be characterized from our knowledge of $M$ and $M T$. For example at low frequencies, $M_{c} \approx M_{\text {min }}$ and $M T_{a b r} \approx M T_{u n i} \approx M T_{m i x}$, so the transmissions are similar and they only depend on the acoustic mismatch between contacts. Unfortunately most of the spectrum is outside this low frequency regime.

For the crystals with scalar IFCs over the mid-frequency range, $M T_{u n i}>M T_{m i x}>M T_{a b r}$ as long as $M_{c} \approx M_{\min }$ (Fig. 5.3a and 5.4a). Therefore, the transmission loss due to disorder for modes that conserve $k_{\perp}$ dominates the transmission gain from modes that do not conserve $k_{\perp}$. As frequency increases, thermal energy is carried by shorter wavelength phonons and disorder back scattering accentuates. At some point, it becomes strong enough to reduce $M T_{m i x}$ even below $M T_{a b r}$ (Fig. 5.4a).

For the DC crystal, the different polarizations available influence the transmission function. For instance, mixing facilitates transmissions between TA-LA modes (Fig. 5.5). This follows from $M T_{m i x}>M T_{u n i}$ in regions where $M_{m i n} \approx M_{c}$, which implies $T_{m i x}>T_{u n i}$. Note that this is the opposite of what we saw for the scalar crystals. Another interesting example shows shifting of transmission between polarizations. Around $\omega \approx 5.2 \times 10^{13} \mathrm{rad} \mathrm{s}^{-1}, M T_{m i x}$ for LA-LO decreases while $M T_{m i x}$ for LA-TO increases. At this frequency the ratio $M_{m i n} / M_{c}$ for LA-TO is larger than for LA-LO so $M T_{m i x}$ shifts towards the more favorable condition 
while conserving energy. In this paper we just scratch the surface of the importance of understanding polarization for interfacial transport, and further studies are required in the topic.

To gain further insights into the transmission, we focus on the crystals with scalar IFCs. For uniform interfaces, Fig. 5.2 shows a conductance maximum when the junction mass is the arithmetic mean (AM) of the contact masses. This follows from a generalization of the same result in $1 \mathrm{D}$ interfaces with a single atomic junction $[64,6]$. By Fourier transforming the transverse coordinates, our 3D problem decouples into a sum of $1 \mathrm{D}$ chains with IFCs that depend on the transverse wavevector. For each $k_{\perp}$ we assume an incident, reflected and transmitted wave and find their amplitudes by solving the equation of motion for the interfacial atom. The transmission $T_{k_{\perp}}$ follows from the ratio of transmitted over incident current. In this way, $M T$ for the uniform interface is

$$
M T_{u n i}=\sum_{k_{\perp}} T_{k_{\perp}}
$$

with

$$
T_{k_{\perp}}=\frac{4 \Gamma_{k_{\perp}}^{l} \Gamma_{k_{\perp}}^{h}}{\omega^{4} \Delta m^{2}+\left(\Gamma_{k_{\perp}}^{l}+\Gamma_{k_{\perp}}^{h}\right)^{2}} .
$$

$\Gamma_{k_{\perp}}$ is the broadening matrix in NEGF formalism, which reduces to a scalar function when dealing with a single degree of freedom per atom [24]. This quantity is related to the escape rate of a phonon into the contacts and is given by $\Gamma_{k_{\perp}}=2 \omega \rho v_{k_{\perp}}$, with $\rho$ the mass density and $v_{k_{\perp}}$ the frequency dependent group velocity of the mode or subband defined by $k_{\perp}$. The superscript in $\Gamma_{k_{\perp}}$ refers to the light $(l)$ and heavy $(h)$ contacts. $\Delta m=m_{j}-\left(m_{l}+m_{h}\right) / 2$ measures the deviation of the junction mass $m_{j}$ from the AM of the contact masses. Thus, when $m_{j}$ is the AM, each $T_{k_{\perp}}, M T_{u n i}$ and $G_{u n i}^{q}$ are maxima. Note that $G_{u n i}^{q}>G_{a b r}^{q}$ as long as $m_{l}<m_{j}<m_{h}$, since the abrupt interface is recovered when $m_{j}=m_{h}$. Also note that at low frequency, $\omega^{4} \Delta m^{2}<<\Gamma_{l}, \Gamma_{h}$, the transmissions only depend on the acoustic mismatch between contacts.

A similar generalization from its $1 \mathrm{D}$ counterpart $[64,6]$ leads us to conclude that in an abrupt interface where interfacial bonding is the only variable, conductance is maximized when the force constant is the harmonic mean of the contact force constants. In the same fashion, we can generalize other 1D results to 3D interfaces [82].

The conductance maximum derived from Eq. 5.8 is not valid for tensorial IFCs (Fig. 5.2). In that case, the amplitudes of the incident and transmitted waves are related through a matrix equation (Eq. 5.27$) . \Delta m \neq 0$ affects both the denominator and the numerator of the transmission, and therefore there is no clear trend when decreasing $\Delta m$. For instance, $\Delta m \neq 0$ might abate the transmission for some polarizations but enhance the transmission between others.

For mixed interfaces, we can approximate $M T_{m i x}$ starting from Eq. 5.29 (Sec. 5.6.2), the relation between 
incident and transmitted wave amplitudes at the interface. The heart of the approximation lies on finding the inverse of the matrix $\left(\tilde{\Delta}+\tilde{Z}_{B}-\tilde{Z}_{C}\right)^{-1}$, which is a diagonal matrix with tiny off-diagonal elements. These small elements come from Fourier transforming the random mass distribution at the interface. We assume that all these elements are constant, since a random mass distribution contains components in the entire frequency spectrum. Then we estimate their value relating the known real power spectrum with the $k$ space spectrum through Parseval's theorem. Finally, we find the desired inverse using a first order Taylor expansion $\left((A+B)^{-1} \approx A^{-1}-A^{-1} B A^{-1}\right)$. With this information, the sum of the transmissions becomes

$$
\begin{aligned}
& M T_{\text {mix }}=\sum_{k_{\perp}} T_{k_{\perp}, k_{\perp}}+\sum_{k_{\perp} \neq k_{\perp}^{\prime}} T_{k_{\perp}, k_{\perp}^{\prime}}, \\
& T_{k_{\perp}, k_{\perp}}=\frac{4 \Gamma_{k_{\perp}}^{l} \Gamma_{k_{\perp}}^{h}}{\omega^{4}\langle\Delta m\rangle^{2}+\left(\Gamma_{k_{\perp}}^{l}+\Gamma_{k_{\perp}}^{h}\right)^{2}}
\end{aligned}
$$

and

$$
T_{k_{\perp}, k_{\perp}^{\prime}}=\frac{\omega^{4}(1-\alpha) \alpha\left(m_{l}-m_{r}\right)^{2}}{N \Gamma_{k_{\perp}}^{l} \Gamma_{k_{\perp}}^{h}} T_{k_{\perp}, k_{\perp}} T_{k_{\perp}^{\prime}, k_{\perp}^{\prime}}
$$

$\langle\Delta m\rangle$ is the average over the junction masses, $N$ is the number of atoms in the cross section and $\alpha$ is the fraction of heavy atoms at the interface. Equation 5.11 suggests that the transmission between modes that do not conserve $k_{\perp}, T_{k_{\perp}, k_{\perp}^{\prime}}$, is proportional to the square of the difference between the atomic masses of the contacts, $\left(m_{l}-m_{r}\right)^{2}$, to the alloy scattering factor, $(1-\alpha) \alpha$, and to some function of the acoustic properties of the contacts. The equation does not capture the decrease in transmission among the modes that conserve $k_{\perp}$ due to disorder. It also over predicts the contribution from transmissions that do not conserve $k_{\perp}$ and does not capture their asymmetric bias as a function of junction mass (Fig. 5.2). In spite of that, it provides a sense for the expected conductance enhancement by mixing and insight on how to build the transmission between different modes, which is an important step forward towards qualitative understanding of interfacial conductance.

\subsection{Simulation details}

Each interface consists of two contacts joined by a layer of primitive unit cells (Fig. 5.1). We find the interfacial thermal conductance using NEGF and assume that the crystal structure, lattice constant $a$ and IFCs are invariant throughout each system. This commonly used simplification [83, 84, 80, 85] provides an easy way to study thermal conductance through vibrationally mismatched interfaces. Moreover, the simplification is well suited for Si/Ge interfaces because the IFCs of these materials are very similar [43] 
and therefore the difference in atomic mass is a dominant scattering mechanism [78]. The ratio between the atomic masses of the contacts is $3\left(m_{l}=40 \mathrm{amu}\right.$ and $\left.m_{h}=120 \mathrm{amu}\right)$ for all the systems but the diamond crystal, where we use the masses of Si and Ge.

The IFCs for the scalar SC and FCC interfaces are built considering only nearest neighbor interactions described by a force constant of $45 \mathrm{~N} / \mathrm{m}$. Assuming $a=5 \AA$, the thermal conductance for the abrupt interface is given by $G_{a b r}=7.5 \mathrm{MW} \mathrm{m}^{-2} \mathrm{~K}^{-1}$ for $\mathrm{SC}$ and $G_{a b r}=44.3 \mathrm{MW} \mathrm{m}^{-2} \mathrm{~K}^{-1}$ for FCC at a temperature of $300 \mathrm{~K}$. Note that the value for FCC is $\sim 6$ times larger than for SC because the FCC crystal has twice the number of atoms per cross sectional area and its MT is $\sim 3$ times larger (Fig. 5.3a and 5.4a).

For the FCC LJ interfaces, the IFCs are extracted from the Lennard-Jones potential using $\epsilon=0.0503 \mathrm{eV}$, $\sigma=3.37 \AA$ and a cut-off distance of $2.5 \sigma$. This potential generates interactions up to fifth-nearest atomic neighbors and corresponds to an equilibrium lattice constant of $a=5.22 \AA$. The potential is chosen to be identical to that used by English et al. [85] to have a point of reference for benchmarking. In fact, we checked the consistency of our IFCs by comparing the phonon dispersions and densities of states against the reference. The conductance for the abrupt interface is $G_{a b r}=57.8 \mathrm{MW} \mathrm{m}^{-2} \mathrm{~K}^{-1}$ at a temperature of $147 \mathrm{~K}$. Our non-equilibrium molecular dynamics (NEMD) simulations predict a larger $G_{a b r}=97.41 \mathrm{MW} \mathrm{m}^{-2} \mathrm{~K}^{-1}$ at a temperature of $30 \mathrm{~K}$ due to anharmonic transmission of phonons beyond the cut off frequency of the heavy material. Note that very low temperature NEMD results, which are classical and mostly harmonic, should tend to high temperature NEGF results, where the Bose-Einstein distribution approaches the classical limit.

For the DC crystal we use the IFCs from silicon extracted using Quantum Espresso, which is a software package for performing calculations using density functional perturbation theory, that has successfully predicted and matched experimental Kapitza conductance and thermal conductivity without any fitting parameters [25]. In this calculation, we used local density approximation (LDA) of Perdew and Zunger [86] with direct fitting. The cutoff energy for the planewave kinetic energy is 30 Ryd, while the $\mathrm{k}$ sampling is $4 \times 4 \times 4$ with Monkhorst-Pack method. We also considered $4 \times 4 \times 4$ q points when calculating the dynamical matrix. The lattice constant for silicon is found to be $5.398 \AA$. Our parameters were chosen after carefully satisfying convergence tests, and the dispersion of silicon matches the experimental data quite well. For simplicity in the calculations, we only consider interactions up to the second nearest neighbor. Our simulations predict $G_{a b r}=242.5 \mathrm{MW} \mathrm{m}^{-2} \mathrm{~K}^{-1}$ at $300 \mathrm{~K}$. To check our code, we simulate the same interface with IFCs extracted from Stillinger-Weber potential and obtain $G_{a b r}=276.6 \mathrm{MW} \mathrm{m}^{-2} \mathrm{~K}^{-1}$ at $300 \mathrm{~K}$, which is comparable to the $G_{a b r}=280 \mathrm{MW} \mathrm{m}^{-2} \mathrm{~K}^{-1}$ reported by Tian et. al. [43] for the same interface, potential and temperature. Those values are within $15 \%$ of the ones obtained using lattice dynamics and NEMD calculations $G_{a b r} \approx 310 \mathrm{MW} \mathrm{m}^{-2} \mathrm{~K}^{-1}$ at $300 \mathrm{~K}[76,81]$.

All our MT calculations are done in transverse wavevector space $\left(k_{\perp}\right.$-space) to simplify the $3 \mathrm{D}$ problem 
into a sum of 1D independent problems. For the abrupt and uniform interfaces each 1D chain consists of primitive unit cells. For the mixed interfaces we increase the size of the unit cell and randomly choose the atoms at the junction layer according to the desired average mass. The unit cell for SC has 36 atoms, for FCC has 32 atoms, for FCC LJ has 18 atoms and for diamond has 36 atoms. For the diamond crystal we also simulated 16 and 64 atoms and did not see appreciable changes in the results. Based on this, the results for FCC LJ might change less than $5 \%$ if we increase the number of atoms. The $M T$ for scalar SC and FCC agree with the $M T$ obtained using Eq. 5.29. For each mixed interface, we report the average over more than 12 independent calculations and in Fig. 5.2 we also report the standard deviation.

To split the contribution of $M T_{m i x}$ from the modes that conserve and do not conserve $k_{\perp}$ (dashed line in Fig. 5.3a and 5.4a), we find the transmission directly from Eq. 5.29 in a system with $40 \times 40$ atoms in the cross section and periodic boundary conditions. Our results show the average over 12 independent simulations of random distributions of atoms at the junction.

To calculate propagating modes for a contact we simulate an "interface" where the leads and junction are the same material. In this case $T=1$ because there is no interface and $M T=M$. The dispersions in the scalar SC and FCC crystals are simple enough that we found the propagating modes analytically by projecting the frequency iso-surface onto the $k_{x}, k_{y}$ plane.

\subsection{Polarization-Resolved Transmission}

To find the transmission resolved by polarizations we start by 1) choosing a frequency $\omega$ for which we identify all the propagating and evanescent modes of both contacts. This is done by solving a generalized eigenvalue problem as explained by Wang et. al. (Sec. 2.2.2 of [31]). Then we 2) assign a polarization to each of the propagating modes. That is, we find the dispersion branch to which each mode belongs. This is done by moving in small wavevector increments from $k_{0}$, a fixed wavevector where we know the correspondence between eigenvalues (frequencies), eigenvectors (polarizations) and branches, to $k^{\prime}$, the wavevector of the phonon we want to label. In each step we calculate the eigenvectors of adjacent $k$ grid points and project ones into the others. Then according to the maximum projection between eigenvectors we assign a branch to each of the eigenvalues and eigenvectors of the next grid point. Once we assigned a label to each propagating mode we 3) find the response around the interface to an incident mode from the left contact. This is done using the Green's function of the system, which is the impulse response of the system, by exciting the system with a superposition of impulses that resemble the mode. Then we 4) project the part of the response at the right contact onto the modes of that contact. At this point we have the amplitude of the impinging 


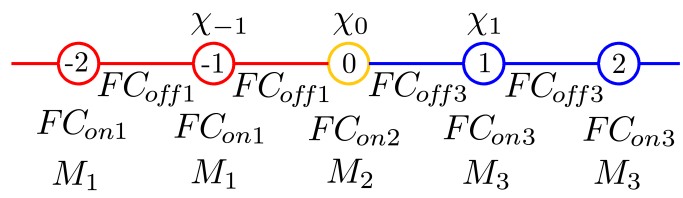

Figure 5.8: System split into sites in the transport direction.

and transmitted modes. Finally, we 5) find the current carried by each mode and the transmission between modes, which we label according to the labels of the modes involved.

For mixed interfaces we have to unfold the dispersion branches of the supercell to be able to identify their polarization and label them consistently with primitive unit cell polarizations. We start this process by expressing a particular eigenvector of the supercell as a linear combination of eigenvectors of primitive unit cells [87]. Then, we find the polarizations of each of the primitive unit cell eigenvectors following the process outlined in step 2. These primitive unit cell eigenvectors have the same polarization. Thus, we assign a unique polarization to the supercell eigenvector and to the corresponding eigenfrequency.

\subsection{Transmission for Mixed Interface}

Our aim is to solve the scattering problem of a wave impinging on an interface to obtain Eq. 5.9, 5.10 and 5.11. To this end we 1) assume incident, reflected and transmitted waves and find an equation relating their amplitudes. Then we 2) approximate that equation to find an analytical solution. Finally we 3) find the transmission from the ratio between transmitted and incident currents and sum them up to get $M T$.

\subsubsection{Equation Relating Amplitudes}

Consider a system split into sites in the transport direction (Fig. B.1) and described by the equation of motion

$$
\omega^{2} M_{s y s} \chi=F_{s y s} \chi
$$

with $M_{\text {sys }}$ the mass matrix of the system

$$
M_{\text {sys }}=\left(\begin{array}{lllll}
\ddots & & & & \\
& M_{1} & & & \\
& & M_{2} & & \\
& & & M_{3} & \\
& & & & \ddots
\end{array}\right) \text {, }
$$


$F_{\text {sys }}$ the force constant matrix of the system

$$
F_{\text {sys }}=\left(\begin{array}{cccccc}
\ddots & & & & \\
& F_{o n 1} & F_{o f f 1} & & \\
& F_{o f f 1}^{\dagger} & F_{o n 2} & F_{o f f 3} & \\
& & F_{o f f 3}^{\dagger} & F_{o n 3} & \\
& & & & \ddots
\end{array}\right)
$$

and $\chi$ the vector containing the displacements from equilibrium of each of the atoms of the system. The equation of motion for the interfacial site is given by

$$
\omega^{2} M_{2} \chi_{0}=F_{o n 2} \chi_{0}+F_{o f f 1}^{\dagger} \chi_{-1}+F_{o f f 3} \chi_{1}
$$

Because of the periodicity of the contacts, plane waves of the form $\chi_{n}=X_{j} e^{i k_{j} n a}$ (Bloch states) satisfy the equation of motion for any contact site if $X_{j}$, the polarization vector, satisfies

$$
\omega^{2} M X_{j}=\left[F_{o n}+F_{o f f}^{\dagger} e^{-i k_{j} a}+F_{o f f} e^{i k_{j} a}\right] X_{j}
$$

In terms of these plane waves we assume a solution for the system of the form

$$
\chi_{n}=\sum_{k_{1}^{+}} A_{k_{1}^{+}} X_{k_{1}^{+}} e^{i k_{1}^{+} n a}+\sum_{k_{1}^{-}} B_{k_{1}^{-}} X_{k_{1}^{-}} e^{-i k_{1}^{-} n a}
$$

for $n \leq 0$, and for $n \geq 0$

$$
\chi_{n}=\sum_{k_{3}^{+}} C_{k_{3}^{+}} Y_{k_{3}^{+}} e^{i k_{3}^{+} n a}
$$

where + and - refer to plane waves propagating to the right or left, $X$ and $Y$ refer to the polarizations on the left and right contacts. We replace the assumed solution (Eq. 5.17 and 5.18) into the equation of motion at the interface (Eq. 5.15). For the factor $F_{o f f 1}^{\dagger} \chi_{-1}$, we split each $F_{o f f 1}^{\dagger} e^{i k_{1}^{ \pm} a}$ into Hermitian and anti-Hermitian parts. We replace the Hermitian part in favor of $\omega^{2} M_{1}-F_{o n 1}$ using Eq. 5.16 and reorganize the anti-Hermitian part in matrix notation to get

$$
F_{o f f 1}^{\dagger} \chi_{-1}=\frac{\omega^{2} M_{1}-F_{o n 1}}{2} \chi_{0}-Z_{A} V_{1+} A-Z_{B} V_{1-} B
$$


with $V_{1+}$ and $V_{1-}$ the matrices whose columns are the polarizations $X_{k_{1}^{+}}$and $X_{k_{1}^{-}}$respectively and with

$$
\begin{gathered}
Z_{A}=\frac{F_{o f f 1} V_{1+} \lambda_{1+} V_{1+}^{-1}-F_{o f f 1}^{\dagger} V_{1+} \lambda_{1+}^{-1} V_{1+}^{-1}}{2}, \\
Z_{B}=\frac{F_{o f f 1} V_{1-} \lambda_{1-} V_{1-}^{-1}-F_{o f f 1}^{\dagger} V_{1-} \lambda_{1-}^{-1} V_{1-}^{-1}}{2}, \\
\lambda_{1 \pm}=\left(\begin{array}{rrr}
e^{i k_{11}^{ \pm} a} & & \\
& e^{i k_{12}^{ \pm} a} & \\
& e^{i k_{13}^{ \pm} a} & \\
& & \ddots
\end{array}\right)
\end{gathered}
$$

where the second subindex of $k_{11}^{ \pm}$run over the possible $k_{1}^{ \pm}$. In a similar way we get that

$$
F_{o f f 1} \chi_{1}=\frac{\omega^{2} M_{3}-F_{o n 3}}{2} \chi_{0}+Z_{C} V_{3+} C
$$

with

$$
Z_{C}=\frac{F_{o f f 3} V_{3+} \lambda_{3+} V_{3+}^{-1}-F_{o f f 3}^{\dagger} V_{3+} \lambda_{3+}^{-1} V_{3+}^{-1}}{2}
$$

Equating Eq. 5.17 and 5.18 at $n=0$ and putting Eq. 5.19 and 5.23 into Eq. 5.15 we get the following set of equations

$$
\begin{gathered}
\chi_{0}=V_{1+} A+V_{1-} B=V_{3+} C \\
\Delta \chi_{0}=-Z_{A} V_{1+} A-Z_{B} V_{1-} B+Z_{C} V_{3+} C
\end{gathered}
$$

with

$$
\Delta=\omega^{2}\left[M_{2}-\frac{M_{1}+M_{3}}{2}\right]-\left[F_{o n 2}-\frac{F_{o n 1}+F_{o n 3}}{2}\right]
$$

From there we can derive the coefficients for the transmitted waves

$$
C=V_{3+}^{-1}\left(\Delta+Z_{B}-Z_{C}\right)^{-1}\left(Z_{B}-Z_{A}\right) V_{1+} A
$$

\subsubsection{Approximation of Amplitudes Equation}

Imagine that each site on Fig. B.1 consists of a cross sectional plane of atoms in the mixed interface (Fig. 5.1b). For the SC and FCC scalar systems, the force constants are invariant in the transport direction, periodic in the transverse direction and scalar between atoms. Thus, $V=V_{1+}=V_{1-}=V_{3+} \cdot V$ is the matrix associated with a Fourier transformation into the transverse $k$-space, whose columns are plane waves defined by $k_{\perp}$ over 
the $N$ atomic positions $r_{n}$ in a cross sectional plane

$$
V=\frac{1}{\sqrt{N}}\left(\begin{array}{ccc}
\mid & \mid & \\
e^{i k_{\perp 1} r_{n}} & e^{i k_{\perp 2} r_{n}} & \ldots \\
\mid & \mid &
\end{array}\right)
$$

Using this information we simplify the relation between the impinging and transmitted waves (Eq. 5.27) as

$$
C=\left(\tilde{\Delta}+\tilde{Z}_{B}-\tilde{Z}_{C}\right)^{-1}\left(\tilde{Z}_{B}-\tilde{Z}_{A}\right) A
$$

where the tilde means the matrix in Fourier space, i.e. $\tilde{Z}_{A}=V^{\dagger} Z_{A} V$. Because of transverse periodicity, all the matrices in Eq. 5.29 are diagonal except

$$
\left[\tilde{M}_{2}\right]_{i, j}=\frac{1}{N} \sum_{n}\left[M_{2}\right]_{n, n} e^{i\left(k_{\perp j}-k_{\perp i}\right) \cdot r}
$$

For $i=j$ the term reduces to the average if the interfacial masses

$$
\left[\tilde{M}_{2}\right]_{i, i}=\left\langle M_{2}\right\rangle=(1-\alpha) m_{l}+\alpha m_{h}
$$

where $\alpha$ is the fraction of heavy atoms at the interfacial layer. For $i \neq j$ we are calculating a frequency component of a random distribution of masses, which should spam over all the $k_{\perp}$ spectrum. Thus we assume that all the off diagonal components of $\tilde{M}_{2}$ have the same magnitude. We estimate the value using Parseval's theorem, the power spectrum in real space and the transformation of the interfacial mass function at $k_{\perp}=0$

$$
\left|\tilde{M}_{i, j}\right|=\sqrt{\frac{(1-\alpha) \alpha}{N-1}}\left|m_{l}-m_{h}\right| .
$$

Plugging this simplification and $Z_{B}=-Z_{A}$ into Eq. 5.29 our problem reduces to solve

$$
C=-2\left[\left(\begin{array}{ccc}
\zeta_{k_{\perp 1}} & \epsilon & \\
\epsilon & \zeta_{k_{\perp 2}} & \\
& & \ddots
\end{array}\right)\right]^{-1}\left(\begin{array}{lll}
\tilde{Z}_{A k_{\perp 1}} & \\
& \tilde{Z}_{A k_{\perp 2}} & \\
& & \ddots
\end{array}\right) A
$$

with

$$
\zeta_{k_{\perp}}=\omega^{2}\left(\left\langle M_{2}\right\rangle-\frac{m_{h}+m_{l}}{2}\right)-\left(\tilde{Z}_{A k_{\perp}}+\tilde{Z}_{C k_{\perp}}\right)
$$




$$
\epsilon=\omega^{2} \sqrt{\frac{(1-\alpha) \alpha}{N-1}}\left|m_{l}-m_{h}\right|
$$

$\epsilon$ is small since it is inversely proportional to $\sqrt{N-1}$, so we approximate the inverse of the matrix using the first order of its Taylor expansion $(A+B)^{-1} \approx A^{-1}-A^{-1} B A^{-1}$ with $A$ being the diagonal part and $B$ the rest. Finding the inverse and solving Eq. 5.33 we get that

$$
C=Q A
$$

with

$$
Q_{l n}= \begin{cases}\frac{-2 Z_{A k_{\perp n}}}{\zeta_{k_{\perp n}}} & \text { if } l=n \\ \frac{\epsilon 2 Z_{A k_{\perp n}}}{\zeta_{k_{\perp l}} \zeta_{k_{\perp n}}} & \text { if } l \neq n\end{cases}
$$

Where $Q_{l n}$ relates the amplitude $A_{n}$ of the $n$ incident mode with amplitude $C_{l}$ of the $l$ transmitted mode.

\subsubsection{Find the Transmission}

Now that we know the coefficients we can calculate the transmission from mode $A_{n}$ to mode $C_{l}$ by dividing the transmitted by the incident current $[6,82]$

$$
T_{l n}=\frac{\Gamma_{r k_{l}}}{\Gamma_{l k_{n}}}\left|\frac{C_{l}}{A_{n}}\right|^{2}=\frac{\Gamma_{r k_{l}}}{\Gamma_{l k_{n}}}\left|M_{l n}\right|^{2}
$$

to obtain

$$
T_{l n}= \begin{cases}\frac{\Gamma_{l k_{\perp n}} \Gamma_{r k_{\perp n}}}{\left|\zeta_{k_{\perp n}}\right|^{2}} & \text { if } l=n \\ \frac{\epsilon^{2} \Gamma_{l k_{\perp n}} \Gamma_{r k_{\perp l}}}{\left|\zeta_{k_{\perp l}}\right|^{2}\left|\zeta_{k_{\perp n}}\right|^{2}} & \text { if } l \neq n\end{cases}
$$

with

$$
\zeta_{k}=\omega^{2}\left[\left\langle m_{n}\right\rangle-\frac{m_{l}+m_{r}}{2}\right]+i\left[\frac{\Gamma_{l k_{n}}}{2}+\frac{\Gamma_{r k_{n}}}{2}\right] .
$$

Here we replace

$$
Z_{A k_{\perp}}=-i \frac{\Gamma_{l k_{\perp}}}{2} \quad Z_{C k_{\perp}}=-i \frac{\Gamma_{r k_{\perp}}}{2}
$$

which is true only for the propagating modes and therefore it works only when both of the modes involved in $T_{l n}$ are propagating, i.e. when $T_{l n} \neq 0$.

Then the $M T$ per unit cell is

$$
M T_{p u c}=\frac{1}{N} \sum_{n} \frac{\Gamma_{l k_{n}} \Gamma_{r k_{n}}}{\left|\zeta_{k_{n}}\right|^{2}}+\frac{1}{N} \sum_{l \neq n} \frac{\epsilon^{2} \Gamma_{l k_{n}} \Gamma_{r k_{l}}}{\left|\zeta_{k_{l}}\right|^{2}\left|\zeta_{k_{n}}\right|^{2}}
$$


and from there Eq. 5.9, 5.10 and 5.11 follow.

\subsection{Summary of Contributions}

We quantify the role of crystal structure and interface morphology on the interface thermal conductance. We show that the crystal structure (SC: simple cubic, FCC: face centered cubic, or DC: diamond cubic) determines the relative magnitude of the minimum of the contacts' modes $M_{\text {min }}$ vs. the conserving modes $M_{c}$ that conserve the component of phonon wavevector transverse to the interface $k_{\perp}$. On the other hand, the interfacial morphology (abrupt, uniform: with an added homogeneous layer, or mixed: with atomic disorder) determines if phonons can move through $M_{c}$ or $M_{m i n}$. Specifically,

1. We find that adding a unit cell monolayer of mixing to an abrupt interface enhances the interfacial conductance, but the degree depends on the ratio $M_{m i n} / M_{c}$.

2. For a scalar FCC crystal, where $M_{\text {min }} \approx M_{c}$, we show that the conductance of a mixed interface increases relative to that of an abrupt interface by $13 \%$. This modest enhancement comes from a balance between the new accessible modes and the extra scattering created by disorder.

3. For a SC crystal, we show that the relative conductance increment from the abrupt interface to the mixed interface is $\sim 150 \%$. This large enhancement comes from a region where there are available modes but they do not overlap $\left(M_{\min }>M_{c}=0\right)$ because of a central void of modes in the Brillouin zone.

4. We show that the void appears when the upward shift in the subbands dominate their shrinking as $k_{\perp}$ increases, which happens due to the independence of interlayer coupling from $k_{\perp}$.

5. For a DC crystal, we find that the effect of mixing depends on the polarization. In particular, mixing increases transmissions between TA branches but not between LA branches. The modes for the TA branches present a central void similar to what we saw in the SC crystal. This suggests that materials with modes containing central voids are prone to high conductance enhancement by mixing.

6. We also find that the conductance across a mixed interface does not always increase relative to that at a uniform interface. In fact, it increases for SC and DC but decreases for FCC, and is once again correlated with the ratio $M_{m i n} / M_{c}$. This suggests that the commonly invoked virtual crystal approximation alternatively overestimates or underestimates the effect of interfacial mixing on thermal conductance. 


\section{Chapter 6}

\section{Conductance Across Many}

\section{Boundaries in 3D}

\subsection{Thermal Conductance Varying Layer Mass and Length}

a) Abrupt

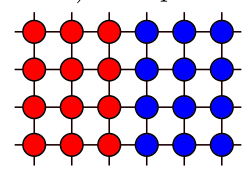

b) Bridged

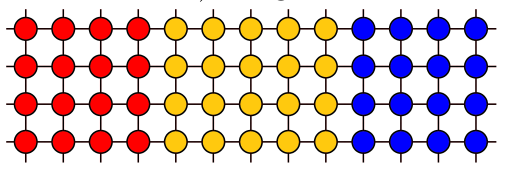

Figure 6.1: Schematic of a) an abrupt interface and b) a bridged interface with an added intermediate layer.

We calculate the thermal conductance of interfaces between two contacts with an added intermediate thin layer (Fig. 6.1b). The layer mass is varied in between the contact masses, whose ratio equals 3 . The length of the layer varies from a couple of atomic layers to tens of atomic layers, so the maximum layer thickness is on the order of tens of nanometers. The interatomic force constants and lattice constants are kept invariant along the system. We simulate two different structures: 1) a face centered cubic (FCC) crystal with interatomic force constants extracted from Lennard-Jones (LJ) potential and contact masses $m_{l}=40$ uma and $m_{r}=120$ uma. And 2) a diamond cubic (DC) crystal with interatomic force constants extracted from Stillinger-Weber (SW) potential [88] and contact masses $m_{l}=28$ uma and $m_{r}=84$ uma. The conductance is calculated from harmonic NEGF as well as from NEMD. Fig. 6.2 and Fig. 6.3 show the results of our calculations for the cases mentioned above.

Our simulations suggest that anharmonicity is a key factor to enhance the abrupt interface conductance by adding a bridging layer. Indeed, the MD results, which include anharmonicity, show a maximum relative 


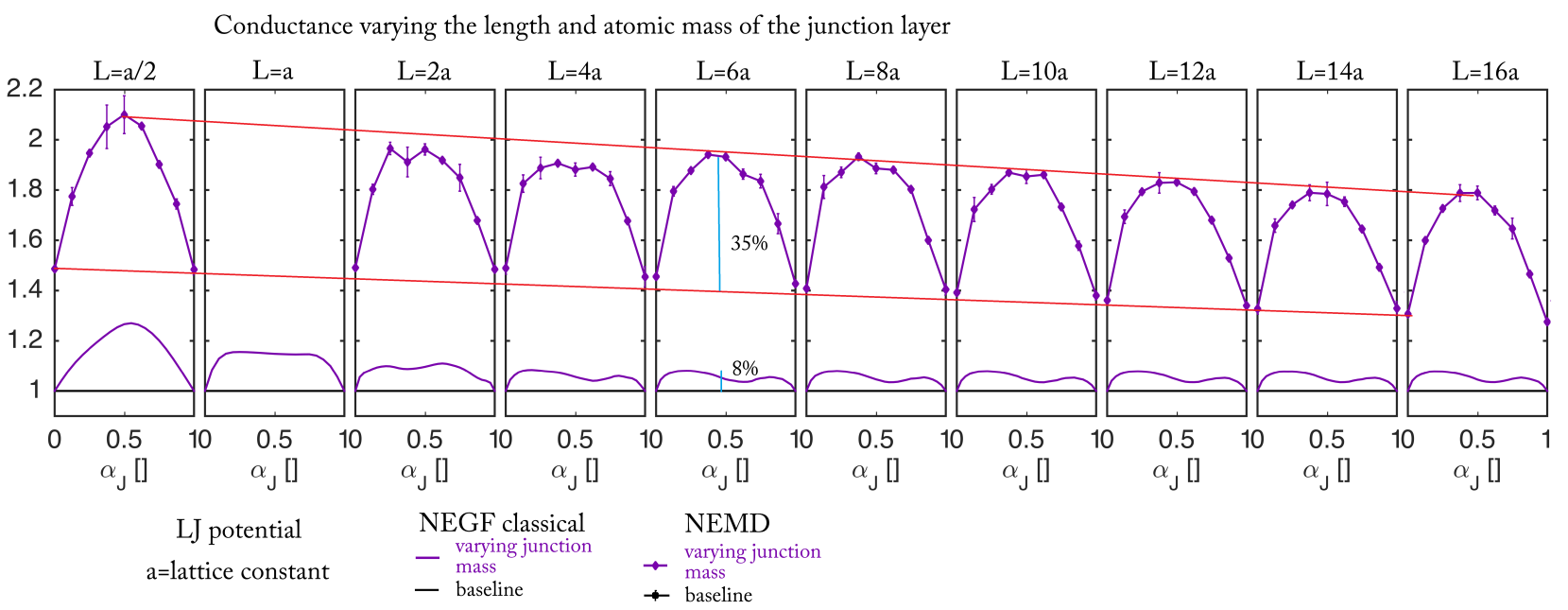

Figure 6.2: Conductance vs. junction atomic mass for different junction lengths. The IFCs come from the LJ interatomic potential. The conductance increases in the harmonic regime only $8 \%$, while the conductance increases when anharmonicity is present by about $33 \%$. This implies that anharmonic processes of phonons are the dominant factor for the enhancement of interfacial conductance, while impedance matching plays a minor role.

increase in conductance over four times larger than the one from NEGF simulations, which is a harmonic calculation. For instance, for a layer of six unit cells, Fig. 6.2 shows a $35.56 \%$ enhancement at $\boldsymbol{T}=30 \mathrm{~K}$ for MD while only a $8.04 \%$ enhancement for NEGF. This is not only due to the expected linear increase in conductance usually seen in MD simulations [cite]. In fact, the extrapolation of the relative maximum conductance enhancement to $\boldsymbol{T}=0 \mathrm{~K}$ (Fig. 6.4) comes close to the NEGF result for very short layers but not for longer layers. This suggests the existence of an additional mechanism that is decreasing the conductance enhancement in the ballistic regime. The mechanism is a decrease in the number of conducting channels brought by an extra constraint on the modes that can conserve transverse momentum (Sec. 6.2).

From the MD results we can make several observations. As the layer length $L$ increases, the conductance curve shifts downwards. The difference between maximum and minimum conductance decreases for short $L$. However, it becomes stable for $L$ longer than 4 unit cells. This suggest that in the long length regime, the explanation for the $G$ vs. $m_{J}$ shape is independent of length. In this regime, we can model the interface resistance as the sum of the boundary resistances plus the layer resistance, where the downward shift as $L$ increases is accounted by layer resistance while the shape of $G$ vs $m_{J}$, including the position of the maximum, depends only on the boundary resistances (Sec. 6.3).

In the short length regime, the NEGF results present a decrease in the difference between maximum and minimum conductance as $L$ increases. This decrease might be related to the analog decrease seen in the MD results because anharmonicity is reduced for lengths shorter than the phonon-phonon scattering length. In 
Conductance varying the length and the atomic mass of the junction

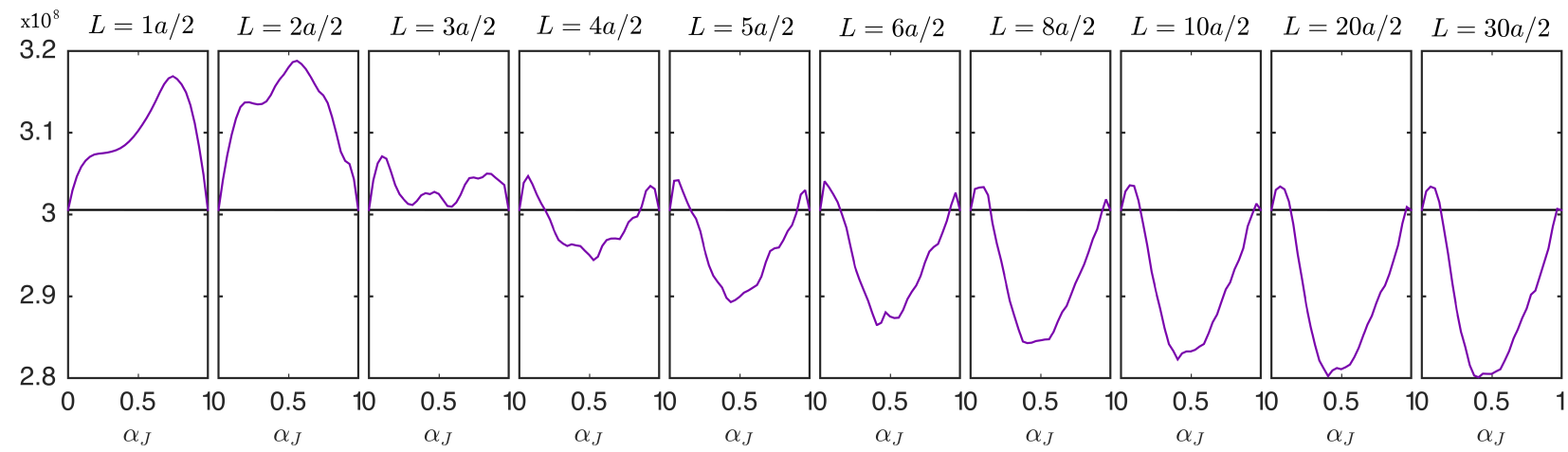

Interface $\mathrm{Si} / \mathrm{VCA} /$ heavy-Si

SW potential

NEGF classical

$\mathrm{G}[\mathrm{W} /(\mathrm{K} \mathrm{m} 2)]$

$a=$ lattice constant

- varying junction

- baseline

Figure 6.3: Conductance vs junction atomic mass for different junction lengths. The IFCs come from the SW interatomic potential. The conductance decreases in the harmonic regime when we add a junction material. This means that impedance matching is not useful to enhance conductance.

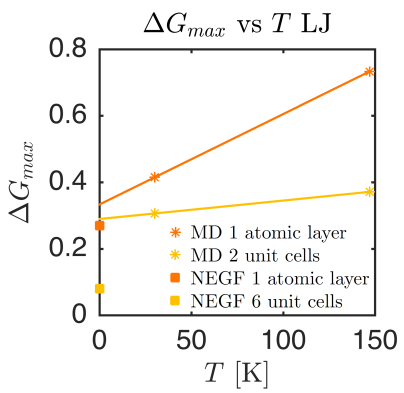

Figure 6.4: Extrapolation of the maximum relative conductance enhancement calculated by MD to $\boldsymbol{T}=0$. The extrapolation comes close to the NEGF result for very short layers but not for longer layers. This suggests the existence of an additional mechanism that is decreasing the conductance enhancement in the ballistic regime. The mechanism is a decrease in the number of conducting channels brought by an extra constraint on the modes that can conserve transverse momentum.

the long length regime, the $G$ vs. $m_{J}$ curves from NEGF are independent of $L$. This can be explained by decomposing the 3D system into decoupled "1D like" subsystems. The transmission for each subsystem is given by an oscillating function whose envelope functions are fixed by the material properties (See Fig. 2 of [82]). As we increase $L$, the frequency of the oscillations increases but the envelopes remain constant. Thus, for $L>L_{0}$, the oscillations are equally averaged by the frequency integral on the conductance (Eq. 2.1).

The $G$ vs. $m_{J}$ curves from NEGF present some wiggles that can be associated with how the average transmission per mode changes as $m_{J}$ changes (Sec. 6.2). On the other hand, the wiggles are not present in the MD simulations. This is the result of a sum of boundary resistances, each one monotonically increasing with increasing mismatch. Finally, note that $G$ for the bridged interface increases with respect to $G$ for the abrupt interface for the LJ potential while it decreases for the SW potential. These conductance results 
follows from two competing effects: a decrease in the number of available modes vs. an increase in the average transmission (Sec. 6.2.).

\subsection{Harmonic limit: Increasing Transmission vs. Decreasing Con- serving Modes}

The idea of adding an intermediate material at an interface to increase its conductance was born from wave mechanics, where the transmission at boundaries increases as the "mismatch" decreases. At particular frequencies, we can even achieve unit transmission when the interface contacts are impedance matched. The decrease of interfacial conductance on Fig. 6.3 shows that this idea is incomplete. In this section we show that the effect on interface conductance by the added intermediate layer results from a competition between an increase in the average transmission due to our initial idea and a decrease in the number of conducting modes that can conserve transverse momentum.

Conductance $G$ (Eqs. 2.1 and ??) is related to the product of the number of modes times the average transmission per mode $M T$, which can be expressed as the sum of all the possible transmissions between propagating modes of the materials

$$
M T=\sum_{k_{\perp L}, k_{\perp J}, k_{\perp R}} T_{k_{\perp L}, k_{\perp J}, k_{\perp R}}
$$

Because the interfaces on our simulations are periodic in the transverse direction, transverse phonon momentum must be conserved. Thus, only transmissions that involve propagating modes with the same perpendicular wavevector are nonzero. Since those are the actual modes contributing to transport, we conveniently express the $M T$ factor as

$$
M T=\sum_{k_{\perp}} T_{k_{\perp}}=\sum_{\substack{k_{\perp} \text { with } \\ T_{k_{\perp}} \neq 0}} T_{k_{\perp}}=M_{c} T_{c}
$$

where $k_{\perp}$ varies over the whole perpendicular Brillouin zone and the number of conserving modes $M_{c}$ and the average transmission over the conserving modes are given by

$$
M_{c}=\sum_{\substack{k_{\perp} \text { with } \\ T_{k_{\perp}} \neq 0}} 1 \quad T_{c}=\frac{1}{M_{c}} \sum_{k_{\perp}} T_{k_{\perp}}
$$

Note that $M_{c}$ depends only on bulk material properties. For instance, for homogeneous materials, $T=1$ and we can calculate the material modes from NEGF as $M=M T=\operatorname{Trace}\left\{\Gamma_{l} G \Gamma_{r} G^{\dagger}\right\}$. With the modes of the 
composing material $M_{L}, M_{J}$ and $M_{R}$, which are functions of both frequency $\omega$ and transverse wavevector $k_{\perp}$, the conserving modes are given by

$$
M_{c}(\omega)=\sum_{k_{\perp}} \min \left(M_{L}\left(\omega, k_{\perp}\right), M_{J}\left(\omega, k_{\perp}\right), M_{R}\left(\omega, k_{\perp}\right)\right)
$$

$M_{c}$ is powerful concept similar in spirit to AMM and DMM, since it describes part of the interface conductance only with knowledge of bulk properties. Thus, conclusions based on $M_{c}$ could easily motivate genomic studies of materials.

To compare the relative change in conductance, conserving modes and transmission of the bridge interface with respect to the abrupt one, let us define the conductance due to the conserving modes as

$$
G_{M_{c}}=\int_{0}^{\infty} \frac{d \omega}{2 \pi} \hbar \omega \frac{\partial N}{\partial T} M_{c}
$$

By multiplying and dividing the actual conductance (Eq. 2.1) by this quantity, we can estimate an average transmission over frequency $\langle T\rangle_{\omega}$

$$
\langle T\rangle_{\omega}=G / G_{M_{c}}
$$

and rewrite the conductance as

$$
G=\int_{0}^{\infty} \frac{d \omega}{2 \pi} \hbar \omega \frac{\partial N}{\partial \boldsymbol{T}} M_{c} T_{c}=G_{M_{c}}\left\langle T_{c}\right\rangle_{\omega}
$$

Now we can define the conductance change of the bridge interface relative to the abrupt interface as

$$
\frac{G}{G_{0}}=\frac{G_{M_{c}}}{G_{M_{c 0}}} \frac{\left\langle T_{c}\right\rangle_{\omega}}{\left\langle T_{c 0}\right\rangle_{\omega}}
$$

where the quantities with a 0 sub index refer to the abrupt interface. $M_{c 0}$ is defined analogous to Eq. 6.4 , but the minimum is taken only over the modes of the contact materials. Note that the relative change in conductance is the product of the relative change in conserving modes times the relative change in the transmission.

For a single boundary, Fig. 6.5 shows that as the mass ratio tend to one, or the "mismatch" decreases, the conductance increases because both the number of conserving modes and the transmission are increasing.

When we add the intermediate material to an abrupt interface, Fig. 6.6 shows that the conductance can either decrease or increase according to the balance between increasing the transmission and decreasing the conserving modes. In another way, conductance depends on the sum over nonzero transmissions. On one side, 


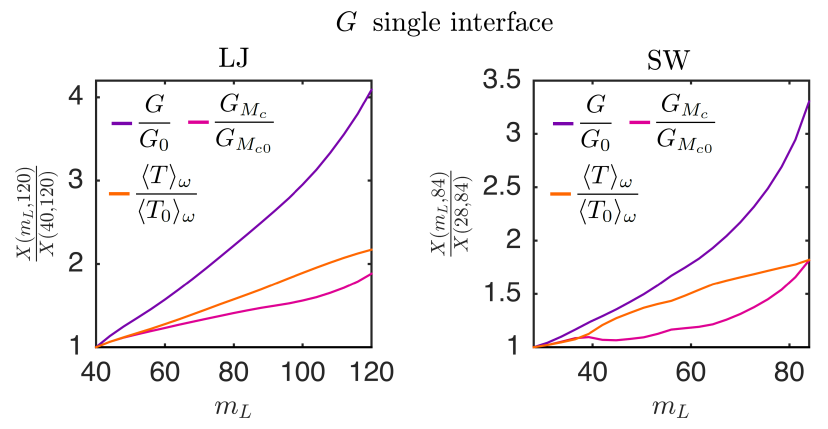

Figure 6.5: For single boundaries, as the ratio between the masses decreases, the interface thermal conductance increases as a result of an increase in both the number of conserving modes and the transmission.

the average transmission increases, but on the other side, the number of nonzero transmissions decreases. For the FCC crystal with LJ potential, the increase in transmission is enough to counter balance the decrease in conserving modes. However, for the DC crystal with SW potential, the decrease in conserving modes dominates and causes an overall decrease in conductance. This could motivate a genomic study of materials to find material combinations that decrease the less the conducting modes. Those combinations should be prone to increase of interface thermal conductance when the layer is added.

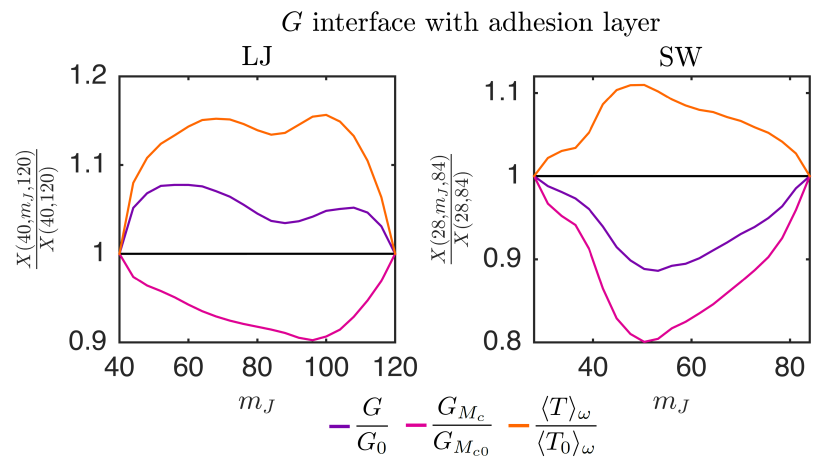

Figure 6.6: For an interface with an intermediate layer, the interface conductance results from an interplay between increasing the transmission due to decreasing the "mismatch" at each boundary and decreasing the number of conserving modes due to a new restriction coming from the intermediate material.

The decrease in the conserving modes arises due to an added constraint. The transverse momentum have to be conserved in three materials instead of two, which implies taking the minimum over tree quantities instead of two (Eq. 6.4). Therefore, in the process of adding an intermediate material, the conserving modes only can decrease from that of the abrupt interface. For instance, Fig. 6.7 shows how the extra constraint decreases the number of modes. 


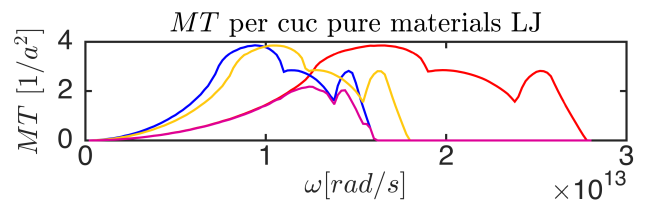

$$
\begin{aligned}
& -M_{m=40} \\
& -M_{m=96} \\
& -M_{m=120} \\
& -M_{c}
\end{aligned}
$$

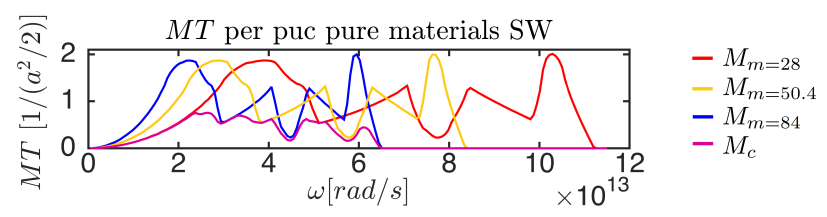

Figure 6.7: The figure shows the available modes for each material and how the adhesion material puts an extra constrain on the conserving modes. Thus hurting $M_{c}$ and decreasing the number of available modes. We plot the cases in which $G_{M_{c}}$ is minimum. (I should probably plot Mc for the abrupt interface also, that will allow a better comparison)

\subsection{Anharmonic limit: Decreasing Boundary Resistance vs. In- creasing Intermediate Layer Resistance}

This section describes how to find the intermediate layer mass that leads to maximum conductance. From MD results in Fig. 6.2 and 6.8a, the maximum $G$ happens for $m_{J}$ between $70 \mathrm{amu}$ and $80 \mathrm{amu}$, when the contacts masses are $m_{L}=40 \mathrm{amu}$ and $m_{R}=120 \mathrm{amu}$. When the contacts masses are $m_{L}=40 \mathrm{amu}$ and $m_{R}=240 \mathrm{amu}$ (Fig. 6.8b), the maximum is around $m_{J}=100 \mathrm{amu}$. Based on these two results, it seems that the $m_{J}$ that maximizes $G$ is close to the geometric mean of the contacts masses.

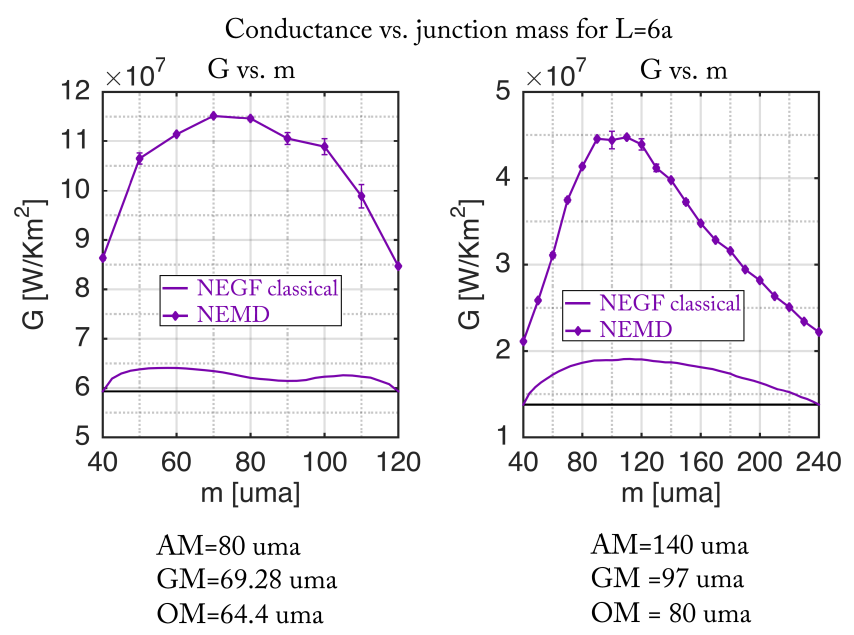

Figure 6.8: Conductance vs. junction atomic mass for two different $m_{r}$. We see a shift in the maximum conductance to the left. Note the non additivity for NEGF of resistances. I bet if I sum the interfaces contributions is different

The geometric mean condition follows because the interface resistance can be decomposed as the sum of the boundary resistances plus the junction resistance; and each boundary resistance increases monotonically as 
the mass ratio increases. To minimize the resistance, we want to minimize the mass ratio at both boundaries at the same time, which best case is when the ratios are equal. Finally, equal mass ratios lead to the GM condition. To prove the point we proceed in the following way: 1) We find out how the single boundary resistance depends on the mass ratio and cut off frequency. 2) We show that the minimum of the sum of individual resistances agrees with the maximum seen in our simulations. 3) We minimize the sum of boundary resistances and find that $m_{J}$ is close to the GM.

We start by dividing Eq. ?? by the minimum cut off frequency of the contacts $\omega_{\min }$ and reorganizing it to define a frequency average of $M T$ per unit area

$$
\frac{G}{\omega_{\min }} \frac{2 \pi}{k B}=\frac{1}{\omega_{\min }} \int_{0}^{\omega_{\max }} d \omega \frac{M T}{A}=\langle M T\rangle_{\omega}
$$

We use $\omega_{\min }$ in the definition to be able to compare with $\langle M T\rangle$ obtained from harmonic NEGF, where transmission is zero beyond $\omega_{m i n}$. Note that if we decide to divide over the maximum frequency instead, it should also works out. In essence, that frequency places the mass ratio in the frequency domain to allow for a conductance calculation.

From each of our MD simulations for bridged interfaces, we extracted two boundary conductances and plot the $\langle M T\rangle_{\omega}$ as a function of the mass ratio in Fig. 6.9a. Since all the data falls under a single curve, we assume that $\langle M T\rangle_{\omega}$ is a function of the mass ratio. Note that the data for the left interface (red) is in general larger than the one for the right interface (blue). This is because the average temperature on the left boundary is larger than that on the right boundary, since $\boldsymbol{T}$ is decreasing from left to right. Thus, $\langle M T\rangle_{\omega}$ is a function only of mass ratio and temperature. This makes sense since the only variables are the masses of the materials. Fig. 6.9a also plots $\langle M T\rangle_{\omega}$ obtained from NEGF. That $\langle M T\rangle_{\omega}$ is bounded because NEGF always includes the contact resistance. When we subtract the contact resistance, the NEGF $\langle M T\rangle_{\omega}$ is an increasing function of the mass ratio, similar to the MD result.

Using Eq. 6.9, we can rewrite $G$ as

$$
G=\frac{k_{B}}{2 \pi A_{c u c}} \omega_{\min }\langle M T\rangle_{A},
$$

where we use the area of the conventional unit cell $A_{c u c}$ to get the $\langle M T\rangle_{\omega}$ per conventional unit cell. With this definition we can write the total resistance

$$
R_{T}=R_{L J}+R_{J}+R_{J R}
$$



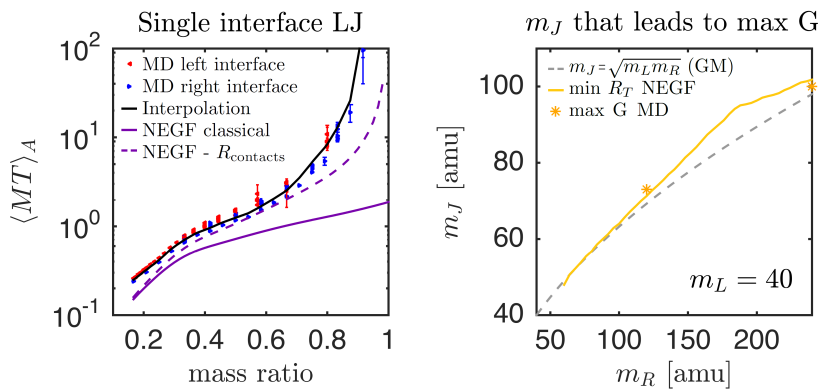

Figure 6.9: $\langle M T\rangle_{A}$ for a single interface is a function of the ratio of the masses only. left this figure shows the $m_{J}$ that lead to minimum resistance calculated from $R_{T}=R_{1}+R_{2}$, the definition of $\mathrm{R}$ as a function of the cutoff frequency and AvgMT that depends on the mass ratio. This prediction based on single junction knowledge is contrasted vs the GM and the maxima from eyeballing from the MD results. Note that the $R_{T}$ are done summing the resistance from NEGF- the contacts resistance. The interpolation from MD have problems because is is not smooth and I get maxima at dirent places. Maybe the interpolation is not guaranteing the minimum increase requirement

as

$$
R_{T}=\frac{2 \pi A_{c u c}}{k_{B} \omega_{c L} \sqrt{m_{L}}}\left[\frac{\sqrt{m_{J}}}{\left\langle M T\left(\frac{m_{L}}{m_{J}}\right)\right\rangle_{A}}+\frac{\sqrt{m_{R}}}{\left\langle M T\left(\frac{m_{J}}{m_{R}}\right)\right\rangle_{A}}\right]+R_{J}
$$

where we have replaced $\omega_{\text {min }}$ with the minimum of the cut off frequencies each boundaries and we have used $\omega_{c J}=\omega_{c L} \sqrt{m_{L} / m_{J}}$ and $\omega_{c R}=\omega_{c L} \sqrt{m_{L} / m_{R}}$, which comes from the fact that the materials are the same except for the mass, so the dispersion, DOS and $M$ are copies of the same function expanded or contracted in the frequency domain.

Now to find the minimum resistance we derivate with respect to $m_{J}$ so

$$
\begin{aligned}
\frac{\partial R_{T}}{\partial m_{J}}= & \frac{1}{2 \sqrt{m_{J}} M T\left(\frac{m_{L}}{m_{J}}\right)}-\sqrt{m_{J}}\left(\frac{1}{M T\left(\frac{m_{L}}{m_{J}}\right)}\right)^{\prime} \frac{m_{L}}{m_{J}^{2}} \\
& +\sqrt{m_{R}}\left(\frac{1}{M T\left(\frac{m_{J}}{m_{R}}\right)}\right)^{\prime} \frac{1}{m_{R}}=0
\end{aligned}
$$

If we neglect the first term, assume that $\sqrt{m_{J}} \approx \sqrt{m_{R}}$ and assume that the derivative is large, the GM will make the derivative the same and at the same time cancel the remaining factors. The exact solution is shown in Fig. 6.9b, where we can it is close to the GM.

\subsection{Summary of Contributions}

- In the harmonic regime, the addition of the layer can either increase or decrease the interface conductance according to which of two competing effects dominates. On one hand, (1) average phonon transmission increases due to an improved matching of the contact impedances. On the other hand, (2) the number 
of available conduction channels decreases because the layer adds an extra constraint on the set of modes that can conserve transverse momentum.

- In the anharmonic regime, the added layer increases the interface conductance if the decrease of resistance at the contact-layer boundaries surpasses the increase of resistance by the layer itself. Moreover, the conductance maximizes when the mismatch at the contact-layer boundaries is equal. For the particular systems simulated in this work, where only the atomic mass changes, the boundary resistance depends predominantly on the mass ratio. Thus, maximum conductance happens when the layer mass is close to the geometric mean of the contact masses, since this condition equate the boundaries mass ratios. 


\section{Chapter 7}

\section{Conclusions and Future work}

\subsection{Conclusions}

In this work we study phonon transport across interfaces with single and multiple boundaries varying the dimensionality, the crystal structure, the interface morphology and the transport regime. In the process we have developed the infrastructure to simulate phonon transport using non-equilibrium Green's functions in the harmonic and anharmonic regimes, coupled with interatomic force constants that can come from interatomic potentials as well as from first principles. We also develop various codes to find the transmission resolved by polarization and many relevant solid states quantities including DOS and dispersion. Combining Landauer theory with NEGF, we have gained insights on the processes determining phonon flow in the different interfaces studied. We have also provided rules for choosing the intermediate layer to be added to an interface to maximize its thermal conductance in the various interfaces and limits studied. Overall, the most important contributions of this document are 1) the connection between the broadening matrix and the impedance, which have the potential to easily allow transfer of knowledge between disciplines; and 2) the idea of describing conductance in terms of conserving and minimum modes, which proved very useful in identifying the strength of the conductance enhancement in interfaces with single and double boundaries.

\subsection{Future Work}

Looking ahead, there are many problems left. The most important in my opinion is the generalization of the anharmonic NEGF infrastructure to 3D. This will require a considerable effort on developing formalism and defining the simplifications that will make an anharmonic NEGF simulation for large systems viable. This will have a great impact on the nanoscale heat transfer community, since NEGF present unique advantages 
over other methods (See introduction). For example, this will allow us to find how scattering rates are affected by the finite size of the samples from first principles. The second important problem to tackle is how to combine individual interface conductances to obtain the conductance of a whole system in different transport regimes. This could be combined with generalizations of the impedance concept to create analogies of the scattering matrix theory. The third important problem is to develop simple models to include the role of interfacial properties on the interface thermal conductance. This long standing problem, has not been satisfactory solved yet, but it an important need of the community. 


\section{Chapter 8}

\section{Publications}

- Polanco, C. A.; Rastgarkafshgarkolaei, R.; Zhang, J.; Le, N. Q.; Norris, P. M.; Hopkins, P. E. and Ghosh, A. W.; Role of crystal structure and junction morphology on interface thermal conductance. Phys. Rev. B, American Physical Society, 2015, 92, 144302.

- Polanco, C. A. and Ghosh, A. W.; Enhancing phonon flow through one dimensional interfaces by impedance matching. Journal of Applied Physics, 2014, 116, 083503.

- Polanco, C. A.; Saltonstall, C. B.; Norris, P. M.; Hopkins, P. E. and Ghosh, A. W.; Impedance Matching of Atomic Thermal Interfaces Using Primitive Block Decomposition. Nanoscale and Microscale Thermophysical Engineering. 2013, 17, 263-279.

- Sajjad, R. N.; Polanco, C. A. and Ghosh A. W.; Atomistic deconstruction of current flow in graphene based hetero-junctions. Journal of Computational Electronics, 2013, 12, 232-247.

- Saltonstall, C. B.; Polanco, C. A.; Duda J. C.; Ghosh A. W.; Norris, P. M. and Hopkins, P. E.; Effect of interface adhesion and impurity mass on phonon transport at atomic junctions. Journal of Applied Physics, 2013, 113, 013516.

- Chaurey V.; Polanco C.; Chou C. F. and Swami N.; Floating-electrode enhanced constriction dielectrophoresis for biomolecular trapping in physiological media of high conductivity. Biomicrofluidics. 2012. 6, 012806.

- Chaurey V.; Chiang, P. C.; Polanco, C.; Su, Y. H,; Chou, C. F. and Swami, N.; Interplay of Electrical Forces for Alignment of Sub-100 nm Electrospun Nanofibers on Insulator Gap Collectors. Langmuir. 201026 (24), 19022-19026. 


\section{Appendix A}

\section{NEGF Phonons}

\section{A.1 Problem and Approach: Big Picture}

Imagine a system formed by two huge contacts or phonon reservoirs kept at different thermal equilibriums and connected through a channel. The hotter contact wants to bring the channel to its thermal equilibrium injecting energy carriers into the channel. Contrary, the cooler contact sucks energy carriers from the channel trying to bring it to its thermal equilibrium. This interplay generates a thermal energy flow from the hotter to the cooler contact [24]. Intuitively, this energy flow should depend on the contacts temperature or in other words, on the number of energy carries (phonons) at the contacts. It also depends on how easy a carrier enters or leaves the channel and on how many places are available in the channel to put carriers. Quantifying the former insights within the NEGF formalism is our goal on section A.2.

Non Equilibrium Green's Function (NEGF) formalism is used to find the thermal energy current. This methodology starts by finding the classical phonon or wave $\boldsymbol{\nu}$ formed inside the channel due to the incident classical phonons $\boldsymbol{\mu}_{1}$ and $\boldsymbol{\mu}_{2}$ from the contacts at a particular frequency $\omega$. Then, from the change of energy in time associated with $\boldsymbol{\nu}$, the thermal current due to $\boldsymbol{\mu}_{1}$ and $\boldsymbol{\mu}_{2}$ is calculated. Finally, total thermal current is obtained by adding the contribution of different frequencies weighted by the average occupancy of phonons at the contacts. 


\section{A.2 Transport Formalism}

\section{A.2.1 System Equations: Preliminars}

Imagine two isolated contacts ( 1 and 2$)$ and an isolated channel where the deviations of their atoms from their equilibrium positions are the components of the vectors $\chi_{1}, \chi_{2}$ and $\chi$ respectively. Using Newton's laws, the movement of every atom in contact 1 is described by the matrix equation

$$
M_{R 1} \frac{d^{2} \chi_{1}}{d t^{2}}+K_{R 1} \chi_{1}=0
$$

where $M_{R 1}$ is a diagonal matrix containing the mass of contact 1 atoms and $K_{R 1}$ is a hermitian matrix consisting of force constants coupling neighboring atoms.

Since the dimensions of $\chi_{1}$ are irrelevant in solving equation A.1, $\chi_{1}$ is substituted by $\boldsymbol{\mu}_{1} \propto \boldsymbol{\chi}_{1}$ to facilitate the coming treatment and enfasy that $\boldsymbol{\mu}_{1}$ doesn't have legth dimensions. However $\boldsymbol{\mu}_{1}$ can be thought as analog to $\boldsymbol{\chi}_{1}$ for most purposes. Assuming $\boldsymbol{\mu}_{1}=\boldsymbol{u}_{1} e^{-i \omega t}$, equation A.1 becomes time independent

$$
\left[\omega^{2} M_{R 1}-K_{R 1}\right] \boldsymbol{u}_{1}=0 .
$$

Finding an orthonormal basis of eigenvectors (normal modes or classical phonons) of equation A.2, which is an important feature to develop the formalism, is not possible in general because $M_{R 1}^{-1} K_{R 1}$ is not necessarily hermitian. To get around this, the normal modes of equation A.2 $\left\{\boldsymbol{u}_{\alpha 1}\right\}$ are defined from the orthonormal basis $\left\{\boldsymbol{u}_{\alpha 1}^{\text {orth }}\right\}$ of eigenvectors of $W_{R 1}^{\text {orth }}=M_{R 1}^{-1 / 2} K_{R 1} M_{R 1}^{-1 / 2}$ as (Appendix A.5)

$$
\boldsymbol{u}_{\alpha 1}=M_{R 1}^{-1 / 2} \boldsymbol{u}_{\alpha 1}^{\text {orth }}
$$

$\boldsymbol{u}_{\alpha 1}^{\text {orth }}$ is chosen dimensionless to facilitate the normalization of the classical phonon energy (Subsection A.2.4). Then, the dimensions of $\boldsymbol{u}_{\alpha 1}$ follow to be $\left[M^{-1 / 2}\right]$.

Similarly, the time independent matrix equations describing contact 2 and channel are

$$
\left[\omega^{2} M_{R 2}-K_{R 2}\right] \boldsymbol{u}_{2}=0 \quad\left[\omega^{2} M-K\right] \boldsymbol{v}=0
$$

and the normal modes of contact $2\left\{\boldsymbol{u}_{\alpha 2}\right\}$ are defined from

$$
\boldsymbol{u}_{\alpha 2}=M_{R 2}^{-1 / 2} \boldsymbol{u}_{\alpha 2}^{\text {orth }}
$$


To assure that the contacts remain in thermal equilibrium, equation A.2 (A.4) is modified with an external excitation or input of phonons $S_{R 1}\left(S_{R 2}\right)$ and a damping or output of phonons $i \eta$. The latter is an infinitesimal positive number that guarantees working with the retarded Green's function.

$$
\begin{aligned}
& {\left[\omega^{2} M_{R 1}-K_{R 1}+i \eta\right] \boldsymbol{u}_{1}=S_{R 1},} \\
& {\left[\omega^{2} M_{R 2}-K_{R 2}+i \eta\right] \boldsymbol{u}_{2}=S_{R 2} .}
\end{aligned}
$$

In thermal equilibrium, the average occupancy of phonons in the contacts follows Bose-Einstein distributions.

$$
N(\omega)=\frac{1}{\exp \left(\frac{\hbar \omega}{k_{B} T}\right)-1}
$$

\section{A.2.2 Scattering Problem}

When the contacts couple with the channel the classical phonons in the contacts $\left(\boldsymbol{u}_{1}\right.$ and $\left.\boldsymbol{u}_{2}\right)$ scatter at the interfaces giving rise to a classical wave inside the channel $(\boldsymbol{v})$ and backscattered classical waves in the contacts $\left(\boldsymbol{v}_{1}\right.$ and $\left.\boldsymbol{v}_{2}\right)$. This scattering problem for phonons of a particular angular frequency $\omega$ is described by the matrix equation

$$
G_{\text {sys }}^{-1}\left\{\begin{array}{c}
\boldsymbol{u}_{1}+\boldsymbol{v}_{1} \\
\boldsymbol{v} \\
\boldsymbol{u}_{2}+\boldsymbol{v}_{2}
\end{array}\right\}=\left\{\begin{array}{c}
S_{R 1} \\
0 \\
S_{R 2}
\end{array}\right\}
$$

where $G_{\text {sys }}^{-1}$ is given by

$$
\left(\begin{array}{ccc}
\text { contact } 1 & \text { channel } & \text { contact } 2 \\
\omega^{2} M_{R 1}-K_{R 1}+i \eta & -\tau_{1}^{\dagger} & 0 \\
-\tau_{1} & \omega^{2} M-K & -\tau_{2} \\
0 & -\tau_{2}^{\dagger} & \omega^{2} M_{R 2}-K_{R 2}+i \eta
\end{array}\right)
$$

$\tau_{1}, \tau_{2}$ represent the coupling between channel and contacts. That is, force constants relating atoms at the interfaces. From the couple equation and using equations A.6 and A.7, $v$ is solved in terms of the contact classical phonons as

$$
\boldsymbol{v}=G S
$$


where the channel Green's function $G$ turns out to be

$$
G=\left[\omega^{2} M-K-\Sigma_{1}-\Sigma_{2}\right]^{-1}
$$

The self energies $\Sigma_{1}$ and $\Sigma_{2}$ are complex non hermitian matrices with the channel size that depend on $\omega$. They represent the effects of contacts into the channel and they are related with the rate at which phonons leave the channel. In fact, $\Sigma$ makes the eigen frequencies complex causing a time exponential decay of $\boldsymbol{\nu}$. The self energies are given by

$$
\Sigma_{1}=\tau_{1} G_{R 1} \tau_{1}^{\dagger} \quad \Sigma_{2}=\tau_{2} G_{R 2} \tau_{2}^{\dagger}
$$

with $G_{R 1}$ and $G_{R 2}$, the Green's function of the contacts, defined by

$$
\begin{aligned}
& G_{R 1}=\left[\omega^{2} M_{R 1}-K_{R 1}+i \eta\right]^{-1} \\
& G_{R 2}=\left[\omega^{2} M_{R 2}-K_{R 2}+i \eta\right]^{-1}
\end{aligned}
$$

Note that although $\Sigma$ has the size of the channel, it comes from huge matrices multiplication and one of the factors is the inverse of giant matrix. To make this computation viable, two suppositions are made about the system. It is assumed that each atom interacts appreciably only with a finite number of neighbors, which makes most of $\tau$ elements equal to 0 . Thus, only a "small" portion of $G_{R}$, called the surface Green's functions $\left(g_{R}\right)$, is required to perform the matrix multiplication. The second assumption is that the contacts are big enough so they are well described as infinite crystals. Then the surface Green's function can be found using a recursive equation (This is explained in more detail in section A.3).

On the other hand, the input to the channel or the excitation from the contacts $S$ is found to be

$$
S=S_{1}+S_{2}=\tau_{1} \boldsymbol{u}_{1}+\tau_{2} \boldsymbol{u}_{2}
$$

and the backscattered phonons are given by

$$
\boldsymbol{v}_{1}=G_{R 1} \tau_{1}^{\dagger} \boldsymbol{v} \quad \boldsymbol{v}_{2}=G_{R 2} \tau_{2}^{\dagger} \boldsymbol{v}
$$

Based on equation A.11, A.12 and A.13, some useful quantities that will be used latter can be defined. The spectral function in the contacts and channel $A_{R 1}, A_{R 2}$ and $A$ which is related to the density of states in 
the contacts and channel (Subsection A.2.7) are defined by

$$
\begin{gathered}
A_{R 1}=i\left(G_{R 1}-G_{R 1}^{\dagger}\right) \quad A_{R 2}=i\left(G_{R 2}-G_{R 2}^{\dagger}\right) \\
A=i\left(G-G^{\dagger}\right)
\end{gathered}
$$

The broadening matrices $\Gamma_{1}$ and $\Gamma_{2}$, which are the antihermitian part of $\Sigma_{1}, \Sigma_{2}$ (Similar to the imaginary part that generates a exponential decay of the amplitude of the waves) and are related with the rate at which classical phonons leave the channel (Subsection A.3.2), are defined by

$$
\begin{aligned}
& \Gamma_{1}=\tau_{1} A_{R 1} \tau_{1}^{\dagger}=i\left(\Sigma_{1}-\Sigma_{1}^{\dagger}\right) \\
& \Gamma_{2}=\tau_{2} A_{R 2} \tau_{2}^{\dagger}=i\left(\Sigma_{2}-\Sigma_{2}^{\dagger}\right)
\end{aligned}
$$

\section{A.2.3 Classical Phonons}

Before calculating the thermal current, the energy of the classical phonon $\boldsymbol{\mu}_{\alpha}=\boldsymbol{u}_{\alpha} e^{-i \omega_{\alpha} t}$ must be normalized to $\hbar \omega_{\alpha}$. Since $\boldsymbol{\mu} \propto \boldsymbol{\chi}$, its energy should look similar to the energy associated with $\chi$ but with a normalization constant. That is

$$
E_{\boldsymbol{\mu}_{\alpha}}=A\left[\frac{1}{2} \dot{\boldsymbol{\mu}}_{\alpha}^{\dagger} M_{R} \dot{\boldsymbol{\mu}}_{\alpha}+\frac{1}{2} \boldsymbol{\mu}_{\alpha}^{\dagger} K_{R} \boldsymbol{\mu}_{\alpha}\right]=\hbar \omega_{\alpha}
$$

Using equations A.2 and A.3 and the orthonormality of $\left\{\boldsymbol{u}_{\alpha}^{\text {orth }}\right\}, A=\hbar / \omega_{\alpha}$. Note that $\hbar$ and $\omega_{\alpha}$ are the two natural constants of the problem.

With all the necessary elements at this point, a classical phonon is defined as

Definition 1. A classical phonon $\boldsymbol{\mu}_{\alpha}=\boldsymbol{u}_{\alpha} e^{-i \omega_{\alpha} t}$, with $\boldsymbol{u}_{\alpha}=M_{s}^{-1 / 2} \boldsymbol{u}_{\alpha}^{\text {orth }}$, is a wave like vector with dimensions $\left[M^{-1 / 2}\right]$ proportional to the "displacement" normal mode $\boldsymbol{\chi}_{\alpha}=\boldsymbol{x}_{\alpha} e^{-i \omega_{\alpha} t}$ of a system defined by $\left[\omega_{\alpha}^{2} M_{s}-K_{s}\right] \boldsymbol{x}=0$. Its associated energy is given by

$$
E_{\boldsymbol{\mu}_{\alpha}}=\frac{\hbar}{\omega_{\alpha}}\left[\frac{1}{2} \dot{\boldsymbol{\mu}}_{\alpha}^{\dagger} M_{s} \dot{\boldsymbol{\mu}}_{\alpha}+\frac{1}{2} \boldsymbol{\mu}_{\alpha}^{\dagger} K_{s} \boldsymbol{\mu}_{\alpha}\right]=\hbar \omega_{\alpha}
$$

From this energy, the thermal current between different degrees of freedom can be found by expressing the change of energy as a divergence of something, which is then defined as thermal current. Expanding the matrix equation, $E_{\boldsymbol{\mu}_{\alpha}}$ can be split in contributions from different degrees of freedom so that the energy 
associated with the $n^{\text {th }}$ degree of freedom is given by [27]

$$
E_{n}=\frac{\hbar}{2 \omega_{\alpha}}\left[\dot{\mu}_{n}^{*} m_{n} \dot{\mu}_{n}-\sum_{m} \mu_{n}^{*} K^{n m} \mu_{m}\right]
$$

where $m$ varies over the degrees of freedom, $K^{n m}$ are the force constants relating different degrees of freedom and $K^{m m}=-\sum_{m} K^{n m}$. Then, using $M \ddot{\boldsymbol{\mu}}=-K \boldsymbol{\mu}$ the change of energy in time is given by

$$
\frac{d E_{n}}{d t}=-\sum_{m} \frac{\hbar}{2 \omega_{\alpha}}\left(\mu_{n}^{*} K^{n m} \dot{\mu}_{m}-\mu_{m}^{*} K^{n m} \dot{\mu}_{n}\right),
$$

which suggests the definition of the thermal current between degrees of freedom $n$ and $m$ as

$$
J_{n m}=\frac{\hbar}{2 \omega_{\alpha}}\left(\mu_{n}^{*} K^{n m} \dot{\mu}_{m}-\mu_{m}^{*} K^{n m} \dot{\mu}_{n}\right)
$$

\section{A.2.4 Thermal Current Carried by Classical Phonons}

The energy associated with a general wave $\boldsymbol{\nu}_{\alpha}=\boldsymbol{v} e^{-i \omega_{\alpha} t}$ representing the superposition of classical phonons of frequency $\omega_{\alpha}$ coming from the contacts is described by

$$
E_{\boldsymbol{\nu}_{\alpha}}=\frac{\hbar}{\omega_{\alpha}}\left[\frac{1}{2} \dot{\boldsymbol{\nu}}^{\dagger} M \dot{\boldsymbol{\nu}}+\frac{1}{2} \boldsymbol{\nu}^{\dagger} K \boldsymbol{\nu}\right]
$$

Thus, the net steady state thermal current incoming into the channel due to classical phonons $\boldsymbol{u}_{\alpha 1}$ and $\boldsymbol{u}_{\alpha 2}$ is given by

$$
\begin{aligned}
I\left(\omega_{\alpha}^{2}\right) & =\frac{d}{d t} E_{\boldsymbol{\nu}_{\alpha}}=\operatorname{Tr}\left\{\frac{d}{d t} E_{\boldsymbol{\nu}_{\alpha}}\right\} \\
& =\frac{\hbar}{2 \omega_{\alpha}} \operatorname{Tr}\left\{\ddot{\boldsymbol{\nu}}_{\alpha}^{\dagger} M \dot{\boldsymbol{\nu}}_{\alpha}+\dot{\boldsymbol{\nu}}_{\alpha}^{\dagger} M \ddot{\boldsymbol{\nu}}_{\alpha}+\dot{\boldsymbol{\nu}}_{\alpha}^{\dagger} K \boldsymbol{\nu}_{\alpha}+\boldsymbol{\nu}_{\alpha}^{\dagger} K \dot{\boldsymbol{\nu}}_{\alpha}\right\}
\end{aligned}
$$

Using Newton's equation for the system

$$
M_{s y s}\left\{\begin{array}{c}
\ddot{\boldsymbol{\mu}}_{\alpha 1}+\ddot{\boldsymbol{\nu}}_{\alpha 1} \\
\ddot{\boldsymbol{\nu}}_{\alpha} \\
\boldsymbol{\mu}_{\alpha 2}+\ddot{\boldsymbol{\nu}}_{\alpha 2}
\end{array}\right\}=-K_{s y s}\left\{\begin{array}{c}
\boldsymbol{\mu}_{\alpha 1}+\boldsymbol{\nu}_{\alpha 1} \\
\boldsymbol{\nu}_{\alpha} \\
\boldsymbol{\mu}_{\alpha 2}+\boldsymbol{\nu}_{\alpha 2}
\end{array}\right\}
$$


with

$$
\begin{aligned}
M_{\text {sys }}= & {\left[\begin{array}{ccc}
M_{R 1} & 0 & 0 \\
0 & M & 0 \\
0 & 0 & M_{R 2}
\end{array}\right] } \\
K_{\text {sys }}= & {\left[\begin{array}{ccc}
K_{R 1} & \tau_{1}^{\dagger} & 0 \\
\tau_{1} & K & \tau_{2} \\
0 & \tau_{2}^{\dagger} & K_{R}
\end{array}\right] }
\end{aligned}
$$

to eliminate the second derivative and exploiting the fact that $\boldsymbol{\nu}_{\alpha}=\boldsymbol{v}_{\alpha} e^{-i \omega_{\alpha} t}, \boldsymbol{\nu}_{\alpha j}=\boldsymbol{v}_{\alpha j} e^{-i \omega_{\alpha} t}, \boldsymbol{\mu}_{\alpha j}=$ $\boldsymbol{u}_{\alpha j} e^{-i \omega_{\alpha} t}$ and $\dot{\boldsymbol{\nu}}_{\alpha}=-i \omega \boldsymbol{\nu}_{\alpha}$, the net incoming thermal current can be split in one incoming current from contact 1 and one from contact 2 which can be further divided into inflow and outflow components.

$$
\begin{aligned}
I\left(\omega_{\alpha}^{2}\right)= & \overbrace{\operatorname{Inflow~}_{1}^{\alpha}+\text { Outflow }_{1}^{\alpha}}^{\text {Incoming current from contact } 1}+\overbrace{\text { Inflow }_{2}^{\alpha}+\text { Outflow }_{2}^{\alpha}}^{\text {Incoming current from contact } 2} \\
= & \underbrace{\frac{\hbar}{2 i} \operatorname{Tr}\left\{\boldsymbol{v}_{\alpha}^{\dagger} \tau_{1} \boldsymbol{u}_{\alpha 1}-\boldsymbol{u}_{\alpha 1}^{\dagger} \tau_{1}^{\dagger} \boldsymbol{v}_{\alpha}\right\}}_{\text {Inflow }_{1}^{\alpha}} \\
& -\underbrace{\frac{\hbar}{2 i} \operatorname{Tr}\left\{\boldsymbol{v}_{\alpha 1}^{\dagger} \tau_{1}^{\dagger} \boldsymbol{v}_{\alpha}-\boldsymbol{v}_{\alpha}^{\dagger} \tau_{1} \boldsymbol{v}_{\alpha 1}\right\}}_{\text {Outflow }_{1}^{\alpha}} \\
& +\underbrace{\frac{\hbar}{2 i} \operatorname{Tr}\left\{\boldsymbol{v}_{\alpha}^{\dagger} \tau_{2} \boldsymbol{u}_{\alpha 2}-\boldsymbol{u}_{\alpha 2}^{\dagger} \tau_{2}^{\dagger} \boldsymbol{v}_{\alpha}\right\}}_{\text {Inflow }_{2}^{\alpha}} \\
& -\underbrace{\frac{\hbar}{2 i} \operatorname{Tr}\left\{\boldsymbol{v}_{\alpha 2}^{\dagger} \tau_{2}^{\dagger} \boldsymbol{v}_{\alpha}-\boldsymbol{v}_{\alpha}^{\dagger} \tau_{2} \boldsymbol{v}_{\alpha 2}\right\}}_{\text {Outflow }_{2}^{\alpha}} .
\end{aligned}
$$

\section{A.2.5 Total Thermal Current}

To find the total thermal current incoming into the channel from contact 1 , the contribution due to the classical phonon $\boldsymbol{u}_{\alpha 1}$, Inflow ${ }_{1}^{\alpha}$, is simplified first. Using $\boldsymbol{v}_{\alpha}=G\left(\omega_{\alpha}\right) \tau_{1} \boldsymbol{u}_{\alpha 1}+G\left(\omega_{\alpha}\right) \tau_{2} \boldsymbol{u}_{\alpha 2}$ (equations A.10 and A.14), $\boldsymbol{u}_{\alpha 1}^{\dagger} \boldsymbol{u}_{\alpha 2}=0, \boldsymbol{u}_{\alpha 2}^{\dagger} \boldsymbol{u}_{\alpha 1}=0$ (because of their localization on a particular contact) and equation A.17, the inflow becomes (Remember $\operatorname{Tr}(A B)=\operatorname{Tr}(B A))$

$$
\text { Inflow }_{1}^{\alpha}=\frac{\hbar}{2} \operatorname{Tr}\left\{\boldsymbol{u}_{\alpha 1}^{\dagger} \tau_{1}^{\dagger} A\left(\omega_{\alpha}\right) \tau_{1} \boldsymbol{u}_{\alpha 1}\right\}
$$


Now adding the contributions from all the classical phonons weighted by their average occupancy the total inflow from contact 1 is given by

$$
\text { Inflow }_{1}=\frac{\hbar}{2} \operatorname{Tr}\left\{\sum_{\alpha} N_{1}\left(\omega_{\alpha}\right) \tau_{1} \boldsymbol{u}_{\alpha 1} \boldsymbol{u}_{\alpha 1}^{\dagger} \tau_{1}^{\dagger} A\left(\omega_{\alpha}\right)\right\} .
$$

Turning the expression into an integral of angular frequency squared using the delta function $\delta\left(\omega^{2}-\omega_{\alpha}^{2}\right)$, the inflow modifies to

$$
\text { Inflow }_{1}=\frac{\hbar}{2} \int d \omega^{2} N_{1} \operatorname{Tr}\left\{\tau_{1}\left[\sum_{\alpha} \boldsymbol{u}_{\alpha 1} \delta\left(\omega^{2}-\omega_{\alpha}^{2}\right) \boldsymbol{u}_{\alpha 1}^{\dagger}\right] \tau_{1}^{\dagger} A\right\}
$$

The summation can be expressed in terms of the contact Green's functions. This process requires the use of an orthonormal basis and is the reason to define the classical phonons $\left\{\boldsymbol{u}_{\alpha 1}\right\}$ and $\left\{\boldsymbol{u}_{\alpha 2}\right\}$ from orthonormal basis $\left\{\boldsymbol{u}_{\alpha 1}^{\text {orth }}\right\}$ and $\left\{\boldsymbol{u}_{\alpha 2}^{\text {orth }}\right\}$. It can be shown that (Appendix A.6 and A.7)

$$
\sum_{\alpha} \boldsymbol{u}_{\alpha 1} \delta\left(\omega^{2}-\omega_{\alpha}^{2}\right) \boldsymbol{u}_{\alpha 1}^{\dagger}=\delta\left(\omega^{2} M_{R 1}-K_{R 1}\right)
$$

and

$$
2 \pi \delta\left(\omega^{2} M_{R 1}-K_{R 1}\right)=i\left(G_{R 1}-G_{R 1}\right)=A_{R 1}
$$

Replacing equations A.21 and A.22 and using equation A.18 the the total inflow from contact 1 to channel becomes

$$
\text { Inflow }_{1}=\frac{\hbar}{2} \int \frac{d \omega^{2}}{2 \pi} N_{1} \operatorname{Tr}\left\{\Gamma_{1} A\right\}=\int d \omega \frac{\hbar \omega}{2 \pi} N_{1} \operatorname{Tr}\left\{\Gamma_{1} A\right\}
$$

Note that the inflow depends on the average available number of energy carries (phonons) at the contact $1 N_{1}$, on how easy a carrier enters or leaves the channel $\Gamma_{1}$ and on how many places are available in the channel to put carriers $A$.

Similarly, to find the total outgoing thermal current from the channel to contact 1 , the contribution due to the classical wave $\boldsymbol{v}_{\alpha 1}$, Outflow ${ }_{1}^{\alpha}$, is simplified first. Using equation A.12, A.15 and A.18, the outflow becomes

$$
\text { Outflow }_{1}^{\alpha}=\frac{\hbar}{2} \operatorname{Tr}\left\{\boldsymbol{v}_{\alpha} \boldsymbol{v}_{\alpha}^{\dagger} \Gamma_{1}\left(\omega_{\alpha}\right)\right\}
$$

Now adding the contributions from all the classical phonons weighted by their average occupancy and using $\boldsymbol{v}_{\alpha}=G\left(\omega_{\alpha}\right) \tau_{1} \boldsymbol{u}_{\alpha 1}+G\left(\omega_{\alpha}\right) \tau_{2} \boldsymbol{u}_{\alpha 2}, \boldsymbol{u}_{\alpha 1}^{\dagger} \boldsymbol{u}_{\alpha 2}=0$ and $\boldsymbol{u}_{\alpha 2}^{\dagger} \boldsymbol{u}_{\alpha 1}=0$, the total outflow to contact 1 is given 
by

$$
\begin{aligned}
& \frac{\hbar}{2} \operatorname{Tr}\left\{\sum_{\alpha} N_{1}\left(\omega_{\alpha}\right) G\left(\omega_{\alpha}\right) \tau_{1} \boldsymbol{u}_{\alpha 1} \boldsymbol{u}_{\alpha 1}^{\dagger} \tau_{1}^{\dagger} G\left(\omega_{\alpha}\right)^{\dagger} \Gamma_{1}\left(\omega_{\alpha}\right)\right\} \\
& +\frac{\hbar}{2} \operatorname{Tr}\left\{\sum_{\alpha} N_{2}\left(\omega_{\alpha}\right) G\left(\omega_{\alpha}\right) \tau_{2} \boldsymbol{u}_{\alpha 2} \boldsymbol{u}_{\alpha 2}^{\dagger} \tau_{2}^{\dagger} G\left(\omega_{\alpha}\right)^{\dagger} \Gamma_{1}\left(\omega_{\alpha}\right)\right\}
\end{aligned}
$$

Turning the expression into an integral of angular frequency squared employing the delta function $\delta\left(\omega^{2}-\omega_{\alpha}^{2}\right)$, the outflow modifies to

$$
\begin{aligned}
& \frac{\hbar}{2} \int d \omega^{2} N_{1} \operatorname{Tr}\left\{G \tau_{1}\left[\sum_{\alpha} \boldsymbol{u}_{\alpha 1} \delta\left(\omega^{2}-\omega_{\alpha}^{2}\right) \boldsymbol{u}_{\alpha 1}^{\dagger}\right] \tau_{1}^{\dagger} G^{\dagger} \Gamma_{1}\right\} \\
& +\frac{\hbar}{2} \int d \omega^{2} N_{2} \operatorname{Tr}\left\{G \tau_{2}\left[\sum_{\alpha} \boldsymbol{u}_{\alpha 2} \delta\left(\omega^{2}-\omega_{\alpha}^{2}\right) \boldsymbol{u}_{\alpha 2}^{\dagger}\right] \tau_{2}^{\dagger} G^{\dagger} \Gamma_{1}\right\}
\end{aligned}
$$

then using equations A.21 and A.22 and later equation A.18, the outflow becomes

$$
\text { Outflow }_{1}=\frac{\hbar}{2} \int \frac{d \omega^{2}}{2 \pi} \operatorname{Tr}\left\{G\left(N_{1} \Gamma_{1}+N_{2} \Gamma_{2}\right) G^{\dagger} \Gamma_{1}\right\}
$$

Defining the inscattering $\Sigma^{i n}$ and the correlation function $G^{n}$ as

$$
\begin{gathered}
\Sigma^{i n}=N_{1} \Gamma_{1}+N_{2} \Gamma_{2} \\
G^{n}=G \Sigma^{i n} G^{\dagger}
\end{gathered}
$$

the total outflow from channel to contact 1 can be written as

$$
\text { Outflow }_{1}=\frac{\hbar}{2} \int \frac{d \omega^{2}}{2 \pi} \operatorname{Tr}\left\{\Gamma_{1} G^{n}\right\}=\int d \omega \frac{\hbar \omega}{2 \pi} \operatorname{Tr}\left\{\Gamma_{1} G^{n}\right\}
$$

Note that the outflow depends on how easy a carrier enters or leaves the channel $\Gamma_{1}$ and on the number of energy carries (phonons) at the channel $G^{n}$. Using equation A.71 (Appendix A.8), the former can be expressed as

$$
G^{n}=A_{1} N_{1}+A_{2} N_{2}
$$

Then, the number of phonons in the channel is proportional to how many places are available in the channel due to contact $i A_{i}$ times how they are occupied $N_{i}$. 
Doing a similar process for contact 2, the inflow from contact 2 to channel and the outflow from channel to contact 2 are given by

$$
\begin{aligned}
& \text { Inflow }_{2}=\frac{\hbar}{2} \int \frac{d \omega^{2}}{2 \pi} N_{2} \operatorname{Tr}\left\{\Gamma_{2} A\right\}=\int d \omega \frac{\hbar \omega}{2 \pi} N_{2} \operatorname{Tr}\left\{\Gamma_{2} A\right\} \\
& \text { Outflow }_{2}=\frac{\hbar}{2} \int \frac{d \omega^{2}}{2 \pi} \operatorname{Tr}\left\{\Gamma_{2} G^{n}\right\}=\int d \omega \frac{\hbar \omega}{2 \pi} \operatorname{Tr}\left\{\Gamma_{2} G^{n}\right\}
\end{aligned}
$$

Finally, adding the inflow and outflow, the thermal current at contact $i$ is given by

$$
I_{i}=\int d \omega \frac{\hbar \omega}{2 \pi} \bar{I}_{i}
$$

with

$$
\bar{I}_{i}=\operatorname{Tr}\left(\Gamma_{i} A\right) N_{i}-\operatorname{Tr}\left(\Gamma_{i} G^{n}\right)
$$

\section{A.2.6 Transmission}

Replacing equation A.27 and $A=A_{1}+A_{2}$ (equation A.71) in equation A.31, $\bar{I}_{1}$ becomes

$$
\bar{I}_{1}=\operatorname{Tr}\left(\Gamma_{1} A_{2}\right)\left(N_{1}-N_{2}\right)=\Xi_{12}\left(N_{1}-N_{2}\right)
$$

where $\Xi_{12}=\operatorname{Tr}\left(\Gamma_{1} A_{2}\right)$ is called the transmission from contact 1 to contact 2 . Similarly, $\bar{I}_{2}$ can be expressed as

$$
\bar{I}_{2}=\operatorname{Tr}\left(\Gamma_{2} A_{1}\right)\left(N_{2}-N_{1}\right)=\Xi_{21}\left(N_{2}-N_{1}\right)
$$

where $\Xi_{21}=\operatorname{Tr}\left(\Gamma_{2} A_{1}\right)$ is the transmission from contact 2 to contact 1 . Since $A_{i}=G \Gamma_{i} G^{\dagger}, \Xi_{12}=\Xi_{21}$ and $\bar{I}_{1}=-\bar{I}_{2}$. This makes physical sense because at steady state the net current from contact 1 to the channel must equal the net current from the channel to contact 2. Thus, a single transmission $\Xi$ can be defined so that the thermal current flowing between contact 1 and contact 2 is given by

$$
I=\frac{\hbar}{2} \int \frac{d \omega^{2}}{2 \pi} \Xi(\omega)\left(N_{1}-N_{2}\right)=\int d \omega \frac{\hbar \omega}{2 \pi} \Xi(\omega)\left(N_{1}-N_{2}\right)
$$

with

$$
\Xi=\operatorname{Tr}\left(\Gamma_{1} A_{2}\right)=\operatorname{Tr}\left(\Gamma_{2} A_{1}\right)=\operatorname{Tr}\left(\Gamma_{1} G \Gamma_{2} G^{\dagger}\right)=\operatorname{Tr}\left(\Gamma_{2} G \Gamma_{1} G^{\dagger}\right)
$$

Equation A.32 relates NEGF with Landauer formalisms [24]. Then, the transmission $\Xi$ can be thought as the usual ratio of transmitted thermal current (from contact 1 to contact 2) over incident thermal current. 


\section{A.2.7 Local Density of States}

The Density Of States (DOS) describes the number of normal modes per unit frequency and is given by

$$
\operatorname{DOS}(\omega)=\sum_{\alpha} \delta\left(\omega-\omega_{\alpha}\right)
$$

This quantity doesn't have any spatial information about the states, which is important for our purposes since our interest focuses on the channel properties. The missing information can be added by weighting the DOS with the amplitude of a unitary normalized wave which is called the Local Density Of States (LDOS)

$$
\operatorname{LDOS}(i, \omega)=\sum_{\alpha}\left|\boldsymbol{u}_{\alpha}^{\text {orth }}(i)\right|^{2} \delta\left(\omega-\omega_{\alpha}\right)
$$

where $\boldsymbol{u}_{\alpha}^{\text {orth }}(i)$ is the $i$ component, referring to a particular position in space, of the orthonormal eigenvector.

The LDOS is just a diagonal element of the spectral function $A$, in real space basis, normalized to account for the change in the $\delta$ function variable (from $\omega^{2}$ to $\omega$ ) and the use of non orthonormal eigenvectors $\left(\boldsymbol{u}_{\alpha}\right.$ to $\left.\boldsymbol{u}_{\alpha}^{\text {orth }}\right)$

$$
\frac{\omega}{\pi} M^{1 / 2} A M^{1 / 2}=\sum_{\alpha} \boldsymbol{u}_{\alpha}^{o r t h} \delta\left(\omega-\omega_{\alpha}\right)\left(\boldsymbol{u}_{\alpha}^{\text {orth }}\right)^{\dagger}
$$

so that

$$
\operatorname{LDOS}(i, \omega)=\left[\frac{\omega}{\pi} M^{1 / 2} A M^{1 / 2}\right]_{i, i}
$$

and

$$
D O S=\operatorname{Tr}\left(\frac{\omega}{\pi} M^{1 / 2} A M^{1 / 2}\right)
$$

Then, it can be seen that the LDOS is proportional to the spectral function.

\section{A.2.8 Summery of Equations}

The net current incoming to the channel from contact $i$ is given by

$$
I_{i}=\int d \omega \frac{\hbar \omega}{2 \pi} \bar{I}_{i}
$$

where

$$
\begin{gathered}
\bar{I}_{i}=\operatorname{Tr}\left(\Gamma_{i} A\right) N_{i}(\omega)-\operatorname{Tr}\left(\Gamma_{i} G^{n}\right) \\
A=i\left(G-G^{\dagger}\right)
\end{gathered}
$$




$$
\begin{gathered}
G=\left[\omega^{2} M-K-\Sigma_{1}-\Sigma_{2}\right]^{-1} \\
\Sigma_{i}=\tau_{i} G_{R i} \tau_{i}^{\dagger} \\
G_{R i}=\left[\omega^{2} M_{R i}-K_{R i}+i \eta\right]^{-1} \\
\Gamma_{i}=i\left(\Sigma_{i}-\Sigma_{i}^{\dagger}\right)=\tau_{i} A_{R i} \tau_{i}^{\dagger} \\
A_{R i}=i\left(G_{R i}-G_{R i}^{\dagger}\right) \\
G^{n}=G \Sigma^{i n} G^{\dagger}=A_{1} N_{1}+A_{2} N_{2} \\
\Sigma^{i n}=\Gamma_{1} N_{1}+\Gamma_{2} N_{2} \\
A_{1}=G \Gamma_{1} G^{\dagger} \quad A_{2}=G \Gamma_{2} G^{\dagger}
\end{gathered}
$$

The current flowing from contact 1 to contact $2\left(I=I_{1}=-I_{2}\right)$ is also given by

$$
I=\int d \omega \frac{\hbar \omega}{2 \pi} \Xi(\omega)\left(N_{1}-N_{2}\right)
$$

with

$$
\Xi=\operatorname{Tr}\left(\Gamma_{1} A_{2}\right)=\operatorname{Tr}\left(\Gamma_{2} A_{1}\right)=\operatorname{Tr}\left(\Gamma_{1} G \Gamma_{2} G^{\dagger}\right)=\operatorname{Tr}\left(\Gamma_{2} G \Gamma_{1} G^{\dagger}\right)
$$

\section{A.3 Transport in 1D Chain}

\section{A.3.1 Setting Up the Problem}

Imagine a $1 \mathrm{D}$ infinite chain of atoms where the interaction between neighbors is described by springs with force constant $K$, which is a $3 \times 3$ matrix representing the second rank tensor character of the quantity. The system (Figure B.1) consists of two contacts, semi-infinite chains characterized by atomic masses $m_{c 1}$ and $m_{c 2}$ and force constants $K^{c 1}$ and $K^{c 2}$ respectively, and a channel containing 3 atoms with masses $m_{1}, m_{2}$ and $m_{3}$ and 2 springs with force constants $K^{12}, K^{23}$. Contact $1(2)$ is coupled to the channel by a spring with force constant $K^{c 1}\left(K^{c 2}\right)$. 


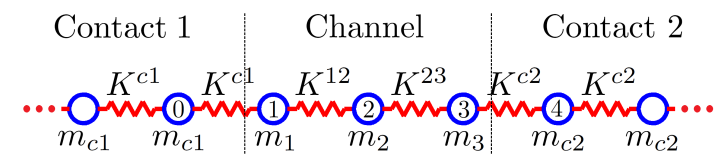

Figure A.1: 1D infinite chain of atoms split in three subsystems to apply NEGF formalism. Two contacts, characterized by atomic masses $m_{c 1}$ and $m_{c 2}$ and force constants $K^{c 1}$ and $K^{c 2}$ respectively, and a channel containing 3 atoms with masses $m_{1}, m_{2}$ and $m_{3}$ and 2 springs with force constants $K^{12}, K^{23}$. Contact $1(2)$ is coupled to the channel by a spring with force constant $K^{c 1}\left(K^{c 2}\right)$.

To extract $M, M_{R 1}, M_{R 2}, K, K_{R 1}, K_{R 2}, \tau_{1}$ and $\tau_{2}$ the system matrix equation must be found first. Thus, the starting point is the time independent Newton's equation for atom $n$

$$
\begin{aligned}
\omega^{2} m_{n} I \boldsymbol{u}_{\boldsymbol{n}}= & \left(K^{n(n+1)}+K^{n(n-1)}\right) \boldsymbol{u}_{n} \\
& -K^{n(n+1)} \boldsymbol{u}_{n+1}-K^{n(n-1)} \boldsymbol{u}_{n-1}
\end{aligned}
$$

where $I$ is the $3 \times 3$ identity matrix and the $3 \times 1$ vector $\boldsymbol{u}_{m}$ is proportional to the deviations from equilibrium of atom $m$. The set of equations can be condensed with matrix notation as

$$
\left[\omega^{2} M_{\text {sys }}-K_{\text {sys }}\right] \boldsymbol{u}=0
$$

where

$$
M_{\text {sys }}=\left[\begin{array}{ccc}
M_{R 1} & & \\
& M & \\
& & M_{R 2}
\end{array}\right], K_{\text {sys }}=\left[\begin{array}{ccc}
K_{R 1} & \tau_{1}^{\dagger} & \\
\tau_{1} & K & \tau_{2} \\
& \tau_{2}^{\dagger} & K_{R 2}
\end{array}\right]
$$

Then, it follows for the particular example that

$$
\begin{gathered}
M=\left[\begin{array}{lll}
m_{1} I & & \\
& m_{2} I & \\
& & m_{3} I
\end{array}\right]_{9 \times 9} \\
M_{R 1}=\left[\begin{array}{lll}
\ddots & & \\
& m_{c 1} I & \\
& & m_{c 1} I
\end{array}\right]_{\infty \times \infty}
\end{gathered}
$$




$$
\begin{aligned}
& M_{R 2}=\left[\begin{array}{lll}
m_{c 2} I & & \\
& m_{c 2} I & \\
& & \ddots
\end{array}\right]_{\infty \times \infty} \\
& K=\left[\begin{array}{cccc}
K^{c 1}+K^{12} & -K^{12} & & \\
-K^{12} & K^{12}+K^{23} & -K^{23} & \\
& -K^{23} & K^{23}+K^{c 2} & -K^{c 2}
\end{array}\right]_{9 \times 9} \\
& K_{R 1}=\left[\begin{array}{ccc}
\ddots & \\
\ddots & 2 K^{c 1} & -K^{c 1} \\
& -K^{c 1} & 2 K^{c 1}
\end{array}\right]_{\infty \times \infty} \\
& K_{R 2}=\left[\begin{array}{ccc}
2 K^{c 2} & -K^{c 2} & \\
-K^{c 2} & 2 K^{c 2} & \ddots \\
& \ddots &
\end{array}\right]_{\infty \times \infty} \\
& \tau_{1}=\left[-K^{c 1}\right]_{9 \times \infty} \tau_{2}=\left[\begin{array}{c} 
\\
-K^{c 2}
\end{array}\right]_{9 \times \infty}
\end{aligned}
$$

Now that the ingredients for the formalism are known, the second aim is to calculate $\Sigma_{1}$ and $\Sigma_{2}$, which are needed to compute $G, \Gamma_{1}$ and $\Gamma_{2}$. Although the size of the self energies is $9 \times 9$, they result from infinite size matrices multiplication with the inverse of an infinite matrix as a factor (Equation A.35).

$$
\begin{aligned}
& {\left[\Sigma_{1}\right]_{9 \times 9}=\left[\tau_{1}\right]_{9 \times \infty}\left[G_{R 1}\right]_{\infty \times \infty}\left[\tau_{1}^{\dagger}\right]_{\infty \times 9}} \\
& {\left[\Sigma_{2}\right]_{9 \times 9}=\left[\tau_{2}\right]_{9 \times \infty}\left[G_{R 2}\right]_{\infty \times \infty}\left[\tau_{2}^{\dagger}\right]_{\infty \times 9}}
\end{aligned}
$$

where the contacts Green's functions $G_{R 1}$ and $G_{R 2}$ are given by equation A.36. To compute this apparently impossible task, two of the premises of the problem turn very useful. It was assumed that each atom interacts appreciably only with a finite number of neighbors, in this particular case 1 . This implies that $\tau$ has only a finite number of elements different from 0 , all of them bunch up on a matrix corner. Thus, only a finite number of elements of $G_{R}$, the surface Green's functions, are required to perform the infinite matrix multiplication. 
Using equation A.47 the self energies simplify to

$$
\Sigma_{1}=\tau_{1} G_{R 1} \tau_{1}^{\dagger}=\left[\begin{array}{l}
K^{c 1} g_{R 1}\left(K^{c 1}\right)^{\dagger} \\
\end{array}\right]_{9 \times 9}
$$

and

$$
\Sigma_{2}=\tau_{2} G_{R 2} \tau_{2}^{\dagger}=\left[\begin{array}{l}
K^{c 2} g_{R 2}\left(K^{c 2}\right)^{\dagger}
\end{array}\right]_{9 \times 9}
$$

where the surface Green's functions $g_{R 1}$ and $g_{R 2}$ are the $3 \times 3$ lower-right and upper-left corners of $G_{R 1}$ and $G_{R 2}$ respectively. To find them, the second favorable assumption comes into play. The contacts are periodic and infinitely long. Therefore, the recursive equations A.74 and A.75 can be used for the computation (Appendix A.9).

$$
g_{R 1}=\left[\alpha-\beta^{\dagger} g_{R 1} \beta\right]^{-1}
$$

with

$$
\alpha=\omega^{2} m_{c 1} I-2 K^{c 1}+i \eta \quad \beta=K^{c 1}
$$

and

$$
g_{R 2}=\left[\alpha-\beta g_{R 2} \beta^{\dagger}\right]^{-1}
$$

with

$$
\alpha=\omega^{2} m_{c 2} I-2 K^{c 2}+i \eta \quad \beta=K^{c 2}
$$

Note that the quadratic equations A.50 and A.51 have two possible solutions. The job of $i \eta$ is to help the numerical recursive solution converge towards the retarded Green's function. Equations A.50 and A.51 are commonly solved using the decimation technique [89]. After the surface Green's functions are calculated, $\Sigma_{1}$, $\Sigma_{2}, G, \Gamma_{1}, \Gamma_{2}, \Xi$ and the thermal current can be computed.

Calculation of transmission and thermal conductance (Explained in Subsection A.4.2) for the particular case in which $m_{c 1}=m_{c 2}=m_{1}=m_{2}=m_{3}=m$ and $K^{c 1}=K^{c 2}=K^{12}=K^{23}=K^{c 1}=K$ with $m=16.6 \times 10^{-27}[\mathrm{~kg}]$ and

$$
K=\left(\begin{array}{ccc}
100 & 0 & 0 \\
0 & 200 & 0 \\
0 & 0 & 300
\end{array}\right)\left[\frac{N}{m}\right]
$$


are shown in FigureA.2. The steps in the transmission correspond to the cut off frequency of the different bands. Indeed, because of the diagonal nature of $K$, this problem is like three decouple 1D chains with scalar force constants. Also, because each 1D chain is homogenous, transmission on each band must be unity.
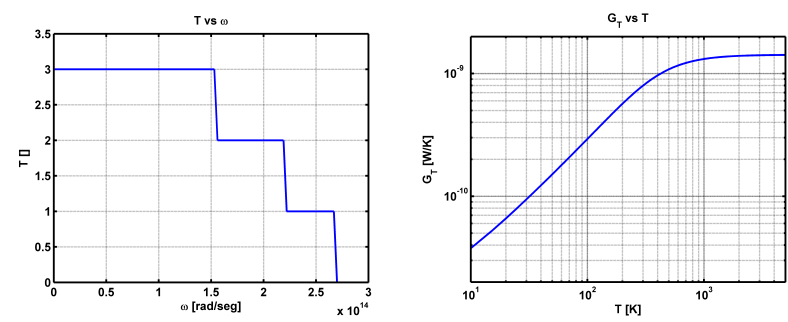

Figure A.2: $1 \mathrm{D}$ infinite chain with same mases $m=16.6 \times 10^{-27}[\mathrm{~kg}]$ and tensor force constants $K$. left) Transmission calculation right) Thermal conductance calculation.

\section{A.3.2 Analytical Example}

The particular 1D chain with equal atoms of mass $m_{0}$, separated by a distance $a$, and springs with scalar force constant $K^{0}$ can be solved analytically. Choosing a single atom channel, to work with scalars instead of matrices, and the contacts as shown in Figure A.3, $M, M_{R 1}, M_{R 2}, K, K_{R 1}, K_{R 2}, \tau_{1}$ and $\tau_{2}$ can be found like in Subsection A.3.1 (Equations A.41-A.47).

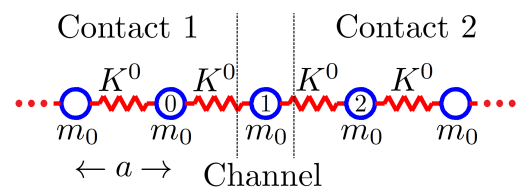

Figure A.3: 1D infinite chain of atoms separated a distance $a$ of mass $m_{0}$ related by springs with scalar force constant $K^{0}$. A single atom channel is chosen to work with scalars instead of matrices.

Based on this information the surface Green's function of contact 1

$$
g_{R 1}=\left[\alpha-\beta^{\dagger} g_{R 1} \beta\right]^{-1}
$$

where

$$
\alpha=\omega^{2} m_{0}-2 K^{0}+i \eta \quad \beta=K^{0}
$$

can be solved analytically using the quadratic formula

$$
g_{R 1}=\frac{\alpha \pm \sqrt{\alpha^{2}-4\left(K^{0}\right)^{2}}}{2\left(K^{0}\right)^{2}}
$$


Noting that i $\eta$ can be dropped as long as the retarded Green's function is picked and using the dispersion relationship in the contact (Equation A.76 Appendix A.10), $\alpha$ satisfies the equality

$$
\alpha=\omega^{2} m_{0}-2 K^{0}=-K^{0}\left(e^{i k a}+e^{-i k a}\right)=-2 K^{0} \cos (k a)
$$

Replacing this into the quadratic solution and choosing the positive exponent result, which refers to the retarded Green's function, $g_{R 1}$ becomes

$$
g_{R 1}=-\frac{e^{i k a}}{K^{0}}
$$

Similarly for contact 2 the surface Green's function turns out to be

$$
g_{R 2}=-\frac{e^{i k a}}{K^{0}}
$$

Now using equation A.48 and A.49, $\Sigma_{1}$ and $\Sigma_{2}$ are given by

$$
\Sigma_{1}=\Sigma_{2}=-K^{0} e^{i k a}
$$

Then the Green's function $G$ can be obtained from equation A.34 and further simplified using equation A.53.

$$
\begin{aligned}
G & =\left[\omega^{2} M-K-\Sigma_{1}-\Sigma_{2}\right]^{-1} \\
& =\frac{1}{\omega^{2} m_{0}-2 K^{0}+2 K^{0} e^{i k a}} \\
& =\frac{1}{2 i K^{0} \sin (k a)}
\end{aligned}
$$

Also, $\Gamma_{1}$ and $\Gamma_{2}$ can be computed replacing equation A.56 in equation A.37 and then simplified using the dispersion relation and the group velocity $v_{g}$ of phonons (equations A.77 and A.78 Appendix A.10) as

$$
\begin{aligned}
\Gamma_{1}=\Gamma_{2} & =2 K^{0} \sin (k a) \\
& =4 K^{0} \sin \left(\frac{k a}{2}\right) \cos \left(\frac{k a}{2}\right) \\
& =2 \omega m_{0} \frac{v_{g}}{a} \\
& =m_{0} \omega \sqrt{\omega_{\max }^{2}-\omega^{2}}
\end{aligned}
$$


Note that $\Gamma_{1}$ is related to the transit time $t_{a}$ of a phonon through the channel

$$
\frac{\Gamma_{1}}{2 \omega m_{0}}=\frac{v_{g}}{a}=\frac{1}{t_{a}}
$$

$\Gamma_{1}$ is also related to the current carried by classical phonons on the contact. In fact, the thermal current from atom $n$ to $n+1$ carried by $\mu_{n}=A e^{i(k n a-\omega t)}$ is found to be (from equation A.19)

$$
J=J_{n, n+1}=\hbar \frac{\Gamma_{1}}{2}|A|^{2}=\hbar \omega v_{g} \frac{m}{a}|A|^{2}
$$

Note that the dimensions of $|A|^{2}$ are $[M]$ according to the definition of $\mu$ (equation A.3) which makes the dimensions of $J$ energy per second. These two observation supports the relation between $\Gamma_{1}$ and the rate at which phonons can enter or leave the channel.

Finally the transmission $\Xi$ within the interval $\left[0, \omega_{\max }\right]$ (Equation A.38) turns out to be

$$
\Xi=\operatorname{Tr}\left(\Gamma_{1} G \Gamma_{2} G^{\dagger}\right)=1
$$

as expected in a single material.

\section{A.4 Transport in 3D Materials}

\section{A.4.1 NEGF on an Infinite Crystalline Material}

If the goal is phonon transport along a particular direction on a system composed of crystals with large cross sectional area, the periodicity perpendicular to the transport direction can be used to split the problem into a set of decoupled 1D problems. Letting $z$ be the transport direction, imagine a system that can be broken into slabs periodic in the perpendicular direction (Figure A.4 left). Assuming that a unit cell $n_{p}$ in slab $z_{p}$
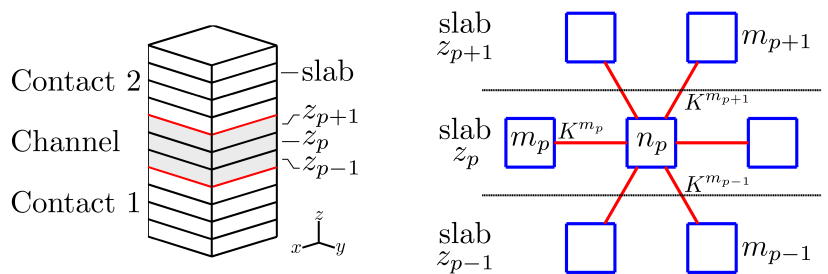

Figure A.4: Left) System broken in slabs perpendicular to the transport direction (z). Right) Schematic representation of the unit cell $n_{p}$ in slab $z_{p} . m_{p}$ are the neighbors of $n_{p}$ in slab $z_{p}$, including itself, $m_{p+1}$ the neighbors of $n_{p}$ in slab $z_{p+1}$ and so on. $K^{m}$ is the block of the force constant matrix $K_{s y s}$ relating $\boldsymbol{u}_{n_{p}}$ with $\boldsymbol{u}_{m}$ 
has interactions $K^{m}$ only with a finite number of its nearest neighbors $m$ (Figure A.4 right), Newton's time independent equation is given by

$$
\begin{aligned}
\omega^{2} M_{n_{p}} \boldsymbol{u}_{n_{p}}= & \sum_{m_{p}} K^{m_{p}} \boldsymbol{u}_{m_{p}}+\sum_{m_{p+1}} K^{m_{p+1}} \boldsymbol{u}_{m_{p+1}} \\
& +\sum_{m_{p-1}} K^{m_{p-1}} \boldsymbol{u}_{m_{p-1}}
\end{aligned}
$$

where $m_{p}$ are the neighbors of $n_{p}$ in slab $z_{p}$, including itself, $m_{p+1}$ the neighbors of $n_{p}$ in slab $z_{p+1}$ and so on. $\boldsymbol{u}_{m}$ is a vector containing the "displacement" information of every atom in unit cell $m$. For instance, if there are 2 atoms per unit cell with 3 degrees of freedom each, $\boldsymbol{u}_{m}$ is a $6 \times 1$ vector. $M_{n_{p}}$ is a matrix containing the masses of all the atoms in the unit cell repeated the number of degrees of freedom per atom and $K^{m}$ is the block of the force constant matrix $K_{s y s}$ relating $\boldsymbol{u}_{n_{p}}$ with $\boldsymbol{u}_{m}$. Since every unit cell of a particular slab sees the same environment, in other words, there is periodicity in the $x y$ plane, postulating Bloch type solutions on the perpendicular direction will simplify the problem. Therefore, assuming $\boldsymbol{u}_{m_{p}}=\tilde{\boldsymbol{u}}_{p} e^{i \boldsymbol{k}_{\perp} \cdot \boldsymbol{d}_{m_{p}}}$, noting that $M_{n_{p}}=M_{m_{p}} \equiv M_{p}$ and defining

$$
\begin{gathered}
\mathbb{M}_{p}=M_{p} \\
\mathbb{K}_{o n}\left(\boldsymbol{k}_{\perp}\right)=\sum_{m_{p}} K^{m_{p}} e^{i \boldsymbol{k}_{\perp} \cdot\left(\boldsymbol{d}_{m_{p}}-\boldsymbol{d}_{n_{p}}\right)} \\
\mathbb{K}_{o f f}\left(\boldsymbol{k}_{\perp}\right)=\sum_{m_{p+1}} K^{m_{p+1}} e^{i \boldsymbol{k}_{\perp} \cdot\left(\boldsymbol{d}_{m_{p+1}}-\boldsymbol{d}_{n_{p}}\right)}
\end{gathered}
$$

equation A.61 becomes

$$
\omega^{2} \mathbb{M}_{p} \tilde{\boldsymbol{u}}_{p}=\mathbb{K}_{o n} \tilde{\boldsymbol{u}}_{p}+\mathbb{K}_{o f f} \tilde{\boldsymbol{u}}_{p+1}+\mathbb{K}_{o f f}^{\dagger} \tilde{\boldsymbol{u}}_{p-1}
$$

which can be compiled into a matrix equation

$$
\omega^{2} \mathbb{M}_{\boldsymbol{k}_{\perp}} \tilde{\boldsymbol{u}}_{\boldsymbol{k}_{\perp}}=\mathbb{K}_{\boldsymbol{k}_{\perp}} \tilde{\boldsymbol{u}}_{\boldsymbol{k}_{\perp}}
$$

with $\mathbb{M}_{\boldsymbol{k}_{\perp}}$

$$
\begin{aligned}
& z_{p-1} \quad z_{p} \quad z_{p+1} \\
& \mathbb{M}_{\boldsymbol{k}_{\perp}}=z_{p-1}\left(\begin{array}{ccccc}
\ddots & & & & \\
& z_{p+1} \\
& \mathbb{M}_{p-1} & & & \\
& & \mathbb{M}_{p} & & \\
& & & \mathbb{M}_{p+1} & \\
& & & \ddots
\end{array}\right)
\end{aligned}
$$


and $\mathbb{K}_{\boldsymbol{k}_{\perp}}$ equal to

$$
\begin{aligned}
& z_{p-1} \quad z_{p} \quad z_{p+1} \\
& z_{p-1}\left(\begin{array}{ccccc}
z_{p} & & & & \\
& \mathbb{K}_{p+1}\left(\boldsymbol{k}_{\perp}\right) & \mathbb{K}_{o f f}\left(\boldsymbol{k}_{\perp}\right) & & \\
& \mathbb{K}_{o f f}^{\dagger}\left(\boldsymbol{k}_{\perp}\right) & \mathbb{K}_{o n}\left(\boldsymbol{k}_{\perp}\right) & \mathbb{K}_{o f f}\left(\boldsymbol{k}_{\perp}\right) & \\
& & \mathbb{K}_{o f f}^{\dagger}\left(\boldsymbol{k}_{\perp}\right) & \mathbb{K}_{o n}\left(\boldsymbol{k}_{\perp}\right) & \\
& & & & \ddots
\end{array}\right)
\end{aligned}
$$

Equation A.65 has a similar form as the 1D problem developed in subsection A.3.1 and it can be solved using a similar procedure. Therefore, for each of this problems $\Sigma_{i}\left(\boldsymbol{k}_{\perp}\right), \Gamma_{i}\left(\boldsymbol{k}_{\perp}\right), G\left(\boldsymbol{k}_{\perp}\right)$ and $\Xi\left(\boldsymbol{k}_{\perp}\right)$ can be found. In that sense, the 3D problem has been decoupled into a set of independent 1D problems, one for each $\boldsymbol{k}_{\perp}$.

The process just done can also be thought as a 2D Fourier Transform of every slab or a change in basis from the $3 \mathrm{D}$ real space basis to a $2 \mathrm{D} \mathrm{k}$ space $+1 \mathrm{D}$ real space basis.

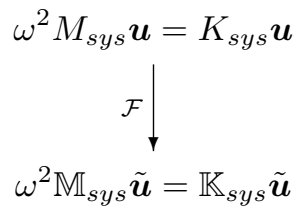

Indeed, if $V$ is the transformation matrix from the $2 \mathrm{D} \mathrm{k}+1 \mathrm{D} z$ space to the $3 \mathrm{D}$ real space then the matrix $M_{\text {sys }}$ is given by

$$
\mathbb{M}_{s y s}=V^{\dagger} M_{s y s} V=M_{\text {sys }}
$$

and becuase of the periodicity of the system, $\mathbb{K}_{\text {sys }}$ becomes block diagonal

$$
\mathbb{K}_{\text {sys }}=V^{\dagger} K_{\text {sys }} V=\left(\begin{array}{lllll}
\ddots & & & & \\
& \mathbb{K}_{\boldsymbol{k}_{\perp 1}} & & & \\
& & \mathbb{K}_{\boldsymbol{k}_{\perp 2}} & & \\
& & & \mathbb{K}_{\boldsymbol{k}_{\perp 3}} & \\
& & & \ddots
\end{array}\right)
$$

It is this block diagonal feature what makes possible to solve the problem as little independents problems or in others words, what decouples the system into a bunch of $1 D$ problems, one for each $\boldsymbol{k}_{\perp}$, described by the equation

$$
\omega^{2} \mathbb{M}_{\boldsymbol{k}_{\perp}} \tilde{\boldsymbol{u}}_{\boldsymbol{k}_{\perp}}=\mathbb{K}_{\boldsymbol{k}_{\perp}} \tilde{\boldsymbol{u}}_{\boldsymbol{k}_{\perp}}
$$

To get the quantities back in real space basis an inverse Fourier transform should be performed. Because 
of the block diagonality of the Green's function in $k$ space, the $(n, m)$ block (relating $\boldsymbol{u}_{n}$ with $\boldsymbol{u}_{m}$ ) of the Green's function is given by

$$
G(n, m)=\frac{1}{N_{k}} \sum_{\boldsymbol{k}_{\perp}} G\left(\boldsymbol{k}_{\perp}\right) \exp \left(i \boldsymbol{k}_{\perp} \cdot\left(\boldsymbol{d}_{m}-\boldsymbol{d}_{n}\right)\right)
$$

where $N_{k}$ is the total number of $\boldsymbol{k}$ points. For the particular calculations in following sections, the interest is $G$ at a single unit cell, therefore

$$
G=G(n, n)=\frac{1}{N_{k}} \sum_{\boldsymbol{k}_{\perp}} G\left(\boldsymbol{k}_{\perp}\right)
$$

The total transmission can be calculated in $k$ space, because the trace of a matrix is independent of the representation. Then, it becomes the summation of the transmission per mode or $\boldsymbol{k}_{\perp}$. That is

$$
\Xi(\omega)=\sum_{\boldsymbol{k}_{\perp}} \Xi\left(\boldsymbol{k}_{\perp}\right)
$$

with

$$
\Xi\left(\boldsymbol{k}_{\perp}\right)=\operatorname{Tr}\left(\Gamma_{l}\left(\boldsymbol{k}_{\perp}\right) G\left(\boldsymbol{k}_{\perp}\right) \Gamma_{r}\left(\boldsymbol{k}_{\perp}\right) G^{\dagger}\left(\boldsymbol{k}_{\perp}\right)\right)
$$

\section{A.4.2 Transport on infinite Silicon along $z$}

Silicon is a FCC crystal (lattice constant $a=5.43 \AA$ ) with two atoms per unit cell of mass $m=4.664 \times 10^{-26} \mathrm{~kg}$ joined by the vector $a / 4(-\hat{\boldsymbol{x}}+\hat{\boldsymbol{y}}+\hat{\boldsymbol{z}})$ (orange bonds in Figure A.5 left). The force constants relating unit cell $n_{0}$ with its neighboring unit cell $m\left(K^{m}\right)$ are calculated from Harrison interatomic potential [90] (Appendix A.11), thus only second nearest interaction between atoms or first nearest neighbor interactions between unit cells are different from zero (Figure A.5).

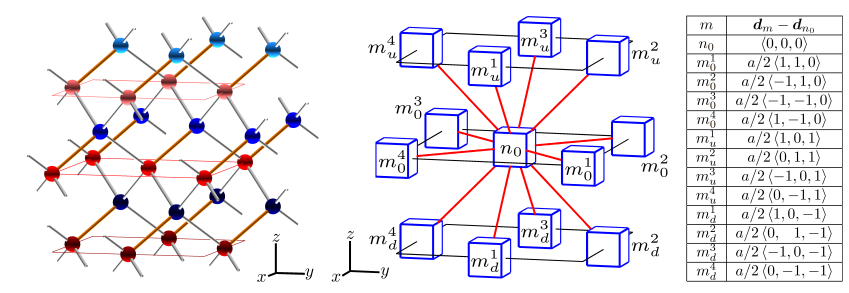

Figure A.5: Si unit cell $n_{0}$ with its nearest units cells. The different $x y$ squares represent different transport slabs. Left) Atom by atom picture. Each reddish atom joined by an orange bond with a bluish atom constitutes a unit cell. The lighter the color of the atom the larger its $z$ component. Middle) Schematic representation of the unit cells Right) Distances between the center unit cell $n_{0}$ and its neighbors $m$

To find the transmission $\boldsymbol{\Xi}$, the periodicity of the crystal in the perpendicular direction ( $x y$ in Figure A.5) is used to decouple the $3 \mathrm{D}$ system into a set of independent $1 \mathrm{D}$ systems (Equation A.67). A set of points 
$\left\{\boldsymbol{k}_{\perp}\right\}$ inside a 2D Brillouin zone in the slab containing unit cell $n_{0}$ is chosen and for each $\boldsymbol{k}_{\perp} \mathbb{K}_{o n}\left(\boldsymbol{k}_{\perp}\right)$ and $\mathbb{K}_{\text {off }}\left(\boldsymbol{k}_{\perp}\right)$ are found using equations A.63 and A.64 and Figure A.5

$$
\begin{aligned}
& \mathbb{K}_{o n}\left(\boldsymbol{k}_{\perp}\right)=K^{n_{0}}+K^{m_{0}^{1}} \exp \left[i \frac{a}{2}\left(k_{x}+k_{y}\right)\right] \\
& +K^{m_{0}^{2}} \exp \left[i \frac{a}{2}\left(-k_{x}+k_{y}\right)\right]+K^{m_{0}^{3}} \exp \left[i \frac{a}{2}\left(-k_{x}-k_{y}\right)\right] \\
& +K^{m_{0}^{4}} \exp \left[i \frac{a}{2}\left(k_{x}-k_{y}\right)\right] \\
& \mathbb{K}_{o f f}\left(\boldsymbol{k}_{\perp}\right)=K^{m_{u}^{1}} \exp \left[i \frac{a}{2}\left(k_{x}\right)\right]+K^{m_{u}^{2}} \exp \left[i \frac{a}{2}\left(k_{y}\right)\right] \\
& +K^{m_{u}^{3}} \exp \left[i \frac{a}{2}\left(-k_{x}\right)\right]+K^{m_{u}^{4}} \exp \left[i \frac{a}{2}\left(-k_{y}\right)\right]
\end{aligned}
$$

Then, the matrices $\mathbb{M}_{\boldsymbol{k}_{\perp}}=m I$, with $I$ a $6 \times 6$ identity matrix and $\mathbb{K}_{\boldsymbol{k}_{\perp}}$ are built as shown bellow equation A.65. With all the necesary components, equation A.65 can be solved in a similar way to the $1 \mathrm{D}$ problem in section A.3. Finally, total transmission is calculated from equation A.67 adding the transmission for each $\boldsymbol{k}_{\perp}$.

Since the thermal current flowing through an infinitely wide material is infinite, the transmission describing that device is also infinite. Thus, to get an useful result that allows comparison with experimental transmission $\Xi$ must be normalized per unit area. This is done by first converting the sum in equation A.67 to an integral assuming periodic boundary conditions

$$
\Xi(\omega)=\frac{A}{4 \pi^{2}} \int_{R} \Xi\left(\boldsymbol{k}_{\perp}\right) d k_{x} d k_{y}
$$

with $R$ the first 2D Brillouin zone in the perpendicular transport direction. Then the integral is approximated numerically as

$$
\Xi(\omega)=\frac{A}{4 \pi^{2}}\left(\begin{array}{c}
\text { Numerical } \\
\Xi
\end{array}\right) \Delta k_{x} \Delta k_{y}
$$

so that the transmission per unit area is given by (Figure A.6)

$$
\frac{\Xi(\omega)}{A}=\frac{1}{4 \pi^{2}}\left(\begin{array}{c}
\text { Numerical } \\
\Xi
\end{array}\right) \Delta k_{x} \Delta k_{y}
$$

Although transmission is an interested theoretical quantity, most experiments measured the thermal conductance

$$
G_{T}=\lim _{\Delta T \rightarrow 0} \frac{I^{Q}}{\Delta T}
$$



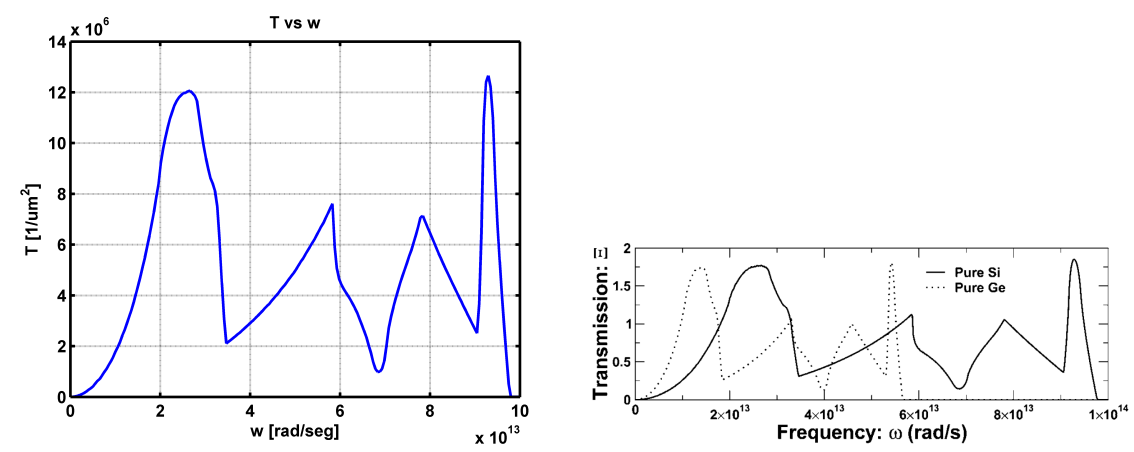

Figure A.6: up) Transmission $\Xi$ per unit area for Silicon calculated from NEGF down) Calculation by [7]

At this limit $(\Delta T \rightarrow 0), \Delta N=N\left(T_{1}\right)-N\left(T_{2}\right)$ can be approximated as

$$
\left.\Delta N \approx \frac{\partial N}{\partial T}\right|_{T_{1}}\left(T_{1}-T_{2}\right)=\frac{1}{T} \frac{x e^{x}}{\left(e^{x}-1\right)^{2}} \Delta T
$$

with $x=\hbar \omega / k_{B} T$. Then, the thermal current (equation A.32) is given by

$$
I^{Q} \approx \frac{k_{B}}{2 \pi} \Delta T \int_{0}^{\infty} d \omega \Xi \frac{x^{2} e^{x}}{\left(e^{x}-1\right)^{2}}
$$

and the thermal conductance becomes (Figure A.7)

$$
G_{T}=\frac{I^{Q}}{\Delta T}=\frac{k_{B}}{2 \pi} \int_{0}^{\infty} d \omega \Xi \frac{x^{2} e^{x}}{\left(e^{x}-1\right)^{2}}
$$
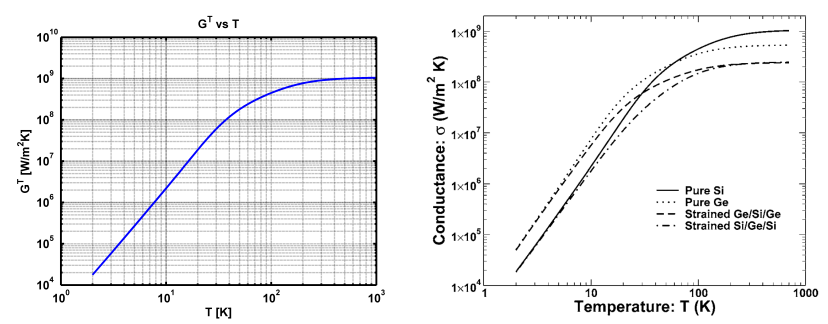

Figure A.7: up) Thermal conductance per unit area (since $\Xi$ is per unit area) for Silicon calculated from NEGF down) Calculation by [7].

\section{A.5 Orthogonalization of equation A.2}

Multiplying on the left by $M_{R 1}^{-1 / 2}$ and inserting the identity $M_{R 1}^{-1 / 2} M_{R 1}^{1 / 2}$ on the left of $\boldsymbol{u}_{1}$, equation A.2 becomes

$$
\left[\omega^{2} I-M_{R 1}^{-1 / 2} K_{R 1} M_{R 1}^{-1 / 2}\right] M_{R 1}^{1 / 2} \boldsymbol{u}_{1}=0
$$


Defining $W_{R 1}^{\text {orth }}=M_{R 1}^{-1 / 2} K_{R 1} M_{R 1}^{-1 / 2}$ and $\boldsymbol{u}_{1}^{\text {orth }}=M_{R 1}^{1 / 2} \boldsymbol{u}_{1}$ the equation turns to

$$
\left[\omega^{2} I-W_{R 1}^{\text {orth }}\right] \boldsymbol{u}_{1}^{\text {orth }}=0
$$

Since $W_{R 1}^{\text {orth }}$ is hermitian an orthonormal basis of eigenvectors $\left\{\boldsymbol{u}_{\alpha 1}^{\text {orth }}\right\}$ can be chosen.

\section{A.6 Summation in Terms of Green's Function for Orthonormal Basis}

Since $\left\{\boldsymbol{u}_{\alpha 1}^{\text {orth }}\right\}$ is the set of eigenvectors of equation A.68 and is a complete orthonormal basis, the sum

$$
\sum_{\alpha} \boldsymbol{u}_{\alpha 1}^{\text {orth }} \delta\left(\omega^{2}-\omega_{\alpha}^{2}\right)\left(\boldsymbol{u}_{\alpha 1}^{\text {orth }}\right)^{\dagger}
$$

can be interpreted as a change of basis of the operator $\delta\left(\omega^{2}-W_{R 1}^{\text {orth }}\right)$ from the orthonormal basis, where the operator is diagonal, to the real space basis. Indeed, defining $V$ as the matrix which columns are the orthonormal vectors $\boldsymbol{u}_{\alpha 1}^{\text {orth }}, V$ represents a unitary transformation from the orthonormal basis to the real space basis. Then

$$
\begin{aligned}
\delta_{r s}\left(\omega^{2}-W_{R 1}^{\text {orth }}\right) & =V \delta_{\text {orth }}\left(\omega^{2}-W_{R 1}^{\text {orth }}\right) V^{\dagger} \\
& =\sum_{\alpha} \boldsymbol{u}_{\alpha 1}^{\text {orth }} \delta\left(\omega^{2}-\omega_{\alpha}^{2}\right)\left(\boldsymbol{u}_{\alpha 1}^{\text {orth }}\right)^{\dagger}
\end{aligned}
$$

The operator $\delta\left(\omega^{2}-W_{R 1}^{\text {orth }}\right)$ can be related with the Green's function. Starting from the equality [24]

$$
\begin{aligned}
2 \pi \delta\left(\omega^{2}-\omega_{\alpha}^{2}\right) & =\left.\frac{2 \eta}{\left(\omega^{2}-\omega_{\alpha}^{2}\right)^{2}+\eta^{2}}\right|_{\eta \rightarrow 0^{+}} \\
& =i\left[\frac{1}{\omega^{2}-\omega_{\alpha}^{2}+i \eta}-\frac{1}{\omega^{2}-\omega_{\alpha}^{2}-i \eta}\right]
\end{aligned}
$$

it can be concluded that in the orthonormal basis representation

$$
\begin{aligned}
2 \pi \delta\left(\omega^{2}-W_{R 1}^{\text {orth }}\right)= & i\left(\left[\omega^{2} I+i \eta-W_{R 1}^{\text {orth }}\right]^{-1}\right. \\
& \left.-\left[\omega^{2} I-i \eta-W_{R 1}^{\text {orth }}\right]^{-1}\right) \\
= & i\left(G_{R 1}^{\text {orth }}-\left(G_{R 1}^{\text {orth }}\right)^{\dagger}\right)
\end{aligned}
$$


where $G_{R 1}^{o r t h}=\left[\omega^{2} I-W_{R 1}^{\text {orth }}+i \eta\right]^{-1}$ is the Greens function of contact 1 in the orthonormal problem. Since the equality holds for the orthonormal representation, it holds in any representation. That is

$$
2 \pi \delta\left(\omega^{2} I-W_{R 1}^{\text {orth }}\right)=i\left(G_{R 1}^{\text {orth }}-\left(G_{R 1}^{\text {orth }}\right)^{\dagger}\right)=A_{R 1}^{\text {orth }}
$$

Combining equations A.69 and A.70 the summation can be expressed in terms of the contact Green's function as

$$
\sum_{\alpha} \boldsymbol{u}_{\alpha 1}^{o r t h} \delta\left(\omega^{2}-\omega_{\alpha}^{2}\right)\left(\boldsymbol{u}_{\alpha 1}^{\text {orth }}\right)^{\dagger}=\frac{A_{R 1}^{o r t h}}{2 \pi}
$$

\section{A.7 Summation in Terms of Green's Function for Non Orthonor- mal Basis}

Using $\boldsymbol{u}_{1}^{\text {orth }}=M_{R 1}^{1 / 2} \boldsymbol{u}_{1}$ and equations A.69 and A.70

$$
\begin{aligned}
\sum_{\alpha} \boldsymbol{u}_{\alpha 1} \delta\left(\omega^{2}-\omega_{\alpha}^{2}\right) \boldsymbol{u}_{\alpha 1}^{\dagger} & =M_{R 1}^{-1 / 2} \delta\left(\omega^{2} I-W_{R 1}^{o r t h}\right) M_{R 1}^{-1 / 2} \\
& =\frac{i}{2 \pi}\left(G_{R 1}-G_{R 1}^{\dagger}\right) \\
& =\frac{A_{R 1}}{2 \pi}
\end{aligned}
$$

The process in appendix A.6 has an analogy for non orthogonal basis noting that the operator $\delta\left(\omega^{2} M_{R 1}-\right.$ $\left.K_{R 1}\right)$ can be expressed in terms of the contact Green's function as

$$
2 \pi \delta\left(\omega^{2} M_{R 1}-K_{R 1}\right)=i\left(G_{R 1}-G_{R 1}^{\dagger}\right)=A_{R 1}
$$

Therefore it can be argued that

$$
\sum_{\alpha} \boldsymbol{u}_{\alpha 1} \delta\left(\omega^{2}-\omega_{\alpha}^{2}\right) \boldsymbol{u}_{\alpha 1}^{\dagger}=\delta\left(\omega^{2} M_{R 1}-K_{R 1}\right)
$$

\section{A.8 A Identity}

Taken from [24] 
Theorem 1. If $G=\left[\omega^{2} M-K-\Sigma_{1}-\Sigma_{2}\right]$ and $\Gamma=\Gamma_{1}+\Gamma_{2}=i\left(\Sigma_{1}-\Sigma_{1}^{\dagger}\right)+i\left(\Sigma_{2}-\Sigma_{2}^{\dagger}\right)$ then

$$
\begin{aligned}
A & =i\left(G-G^{\dagger}\right)=G \Gamma G^{\dagger} \\
& =G \Gamma_{1} G^{\dagger}+G \Gamma_{2} G^{\dagger}=A_{1}+A_{2}
\end{aligned}
$$

Proof. $\left(G^{\dagger}\right)^{-1}-G^{-1}=\Sigma_{1}-\Sigma_{1}^{\dagger}+\Sigma_{2}-\Sigma_{2}^{\dagger}=-i \Gamma$. Then pre-multiplying with $G$ and post-multiplying with $G^{\dagger}$ the equality becomes

$$
G-G^{\dagger}=-i G \Gamma G^{\dagger}
$$

which implies the result.

\section{A.9 Computing Surface Green's Functions}

Assuming the matrices $A, B, C$ and $D$ are unknown and $a, b, c$ and $d$ are known in the matrix equation

$$
\left[\begin{array}{ll}
A & B \\
C & D
\end{array}\right]=\left[\begin{array}{ll}
a & b \\
c & d
\end{array}\right]^{-1},
$$

$A$ and $D$ can be computed as

$$
\begin{aligned}
A & =\left(a-b d^{-1} c\right)^{-1} \\
D & =\left(d-c a^{-1} b\right)^{-1} .
\end{aligned}
$$

In the particular case of

$$
\left[\begin{array}{ll}
A & B \\
C & D
\end{array}\right]=\left[\begin{array}{llll}
\ddots & & & \\
& \alpha & \beta & \\
& \beta^{\dagger} & \alpha & \beta \\
& & \beta^{\dagger} & \alpha
\end{array}\right]^{-1}
$$

with

$$
a=\left[\begin{array}{llll}
\ddots & & & \\
& \alpha & \beta & \\
& \beta^{\dagger} & \alpha & \beta \\
& & \beta^{\dagger} & \alpha
\end{array}\right], b=\left[\begin{array}{l}
\vdots \\
\beta
\end{array}\right], c=[\cdots \cdots
$$

$D$ is given by

$$
D=\left(\alpha-\beta^{\dagger} D \beta\right)^{-1}
$$


In the other particular case

$$
\left[\begin{array}{ll}
A & B \\
C & D
\end{array}\right]=\left[\begin{array}{llll}
\alpha & \beta & & \\
\beta^{\dagger} & \alpha & \beta & \\
& \beta^{\dagger} & \alpha & \\
& & & \ddots
\end{array}\right]^{-1}
$$

with

$$
a=\alpha, b=\left[\begin{array}{ll}
\beta & \ldots
\end{array}\right], c=\left[\begin{array}{c}
\beta^{\dagger} \\
\vdots
\end{array}\right], d=\left[\begin{array}{cccc}
\alpha & \beta & & \\
\beta^{\dagger} & \alpha & \beta & \\
& \beta^{\dagger} & \alpha & \\
& & & \ddots
\end{array}\right]
$$

$A$ is given by

$$
A=\left(\alpha-\beta A \beta^{\dagger}\right)^{-1}
$$

\section{A.10 Dispersion Relation and Group velocity for 1D Analytical Example}

To find the $\omega$ vs $k$ dispersion, the time independent Newton equation for atom $n$ in the system shown in Figure A.3 is the starting point

$$
\omega^{2} m_{0} u_{n}=K^{0}\left(2 u_{n}-u_{n+1}-u_{n-1}\right)
$$

Since the system is periodic spatially, which suggests Bloch waves type solutions, it is supposed that $u_{n}=u_{0} e^{i k n a}$. Then equation turns to

$$
\omega^{2} m_{0} u_{0}=K^{0} u_{0}\left(2-e^{i k a}-e^{-i k a}\right)
$$

or

$$
\omega^{2} m_{0}-2 K^{0}=-K^{0}\left(e^{i k a}+e^{-i k a}\right)=-2 K^{0} \cos (k a)
$$

Solving for $\omega$, the $\omega$ vs $k$ dispersion is obtained (Figure A.8)

$$
\omega=2 \sqrt{\frac{K^{0}}{m_{0}}}\left|\sin \left(\frac{k a}{2}\right)\right|
$$

where only frequencies within the interval $\left[0, \omega_{\max }\right]$ with $\omega_{\max }=2 \sqrt{K^{0} / m_{0}}$ are allowed. 


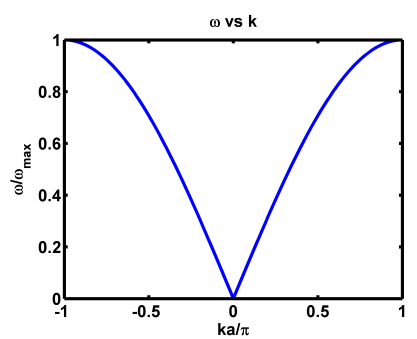

Figure A.8: $\omega$ vs $k$ dispersion of a 1D infinite chain of atoms separated by a distance $a$ of mass $m_{0}$ related by springs with scalar force constant $K^{0}$ (Figure A.3). The maximum allowed angular frequency is $\omega_{\max }=2 \sqrt{K^{0} / m_{0}}$

From the dispersion relation the group velocity of the classical phonons, which is the same group velocity of a packet of planes waves, can be obtained

$$
v_{g}=\frac{d \omega}{d k}= \begin{cases}a \sqrt{\frac{K^{0}}{m_{0}}} \cos \left(\frac{k a}{2}\right) & \text { if } k>0 \\ -a \sqrt{\frac{K^{0}}{m_{0}}} \cos \left(\frac{k a}{2}\right) & \text { if } k<0\end{cases}
$$

Note that although the process was done for an infinite 1D chain, it is also suitable for 1D semi infinite chains. That is, the same results hold for the contacts.

\section{A.11 Si Force Constants from Harrison Potential}

\section{A.11.1 Tensor Force Constants}

Within the harmonic approximation, the force on atom $i$ due to a deviation from equilibrium of atom $j$ can be described in terms of a tensor force constant $K^{i j}$ as $\boldsymbol{F}^{i}=-K^{i j} \boldsymbol{x}^{j}$. The component of this force in the direction $\hat{\boldsymbol{x}}_{p}^{i}$ can be approximated to first order as

$$
F_{x_{p}^{i}} \approx-\sum_{j, q} \frac{\partial^{2} U}{\partial x_{q}^{j} \partial x_{p}^{i}} x_{q}^{j}=-\sum_{j, q} K_{x_{p}^{i}, x_{q}^{j}} x_{q}^{j}
$$

with $U$ the potential energy of the crystal, $x_{p}^{i}, x_{q}^{j}$ degrees of freedom of atom $i, j$ (deviations from equilibrium position). $p$ and $q$ vary over $1,2,3$ and although theoretically $j$ varies over all the atoms in the crystal, the second derivatives decrease quickly as the distance between atoms $i$ and $j$ increases, thus in practice $j$ is taken only over some nearest neighbors of atom $i$. From equation A.79, the force constant relating the total 
force on atom $i$ in the direction $\hat{\boldsymbol{x}}_{p}^{i}$ with a displacement of atom $j$ in the direction $\hat{\boldsymbol{x}}_{q}^{j}$ is given by

$$
K_{x_{p}^{i}, x_{q}^{j}}=\frac{\partial^{2} U}{\partial x_{q}^{j} \partial x_{p}^{i}}
$$

From these individual force constants, the tensor force constant relating the total force on atom $i$ with a displacement of atom $j$ is defined as

$$
K^{i j}=\left(\begin{array}{lll}
K_{x_{1}^{i}, x_{1}^{j}} & K_{x_{1}^{i}, x_{2}^{j}} & K_{x_{1}^{i}, x_{3}^{j}} \\
K_{x_{2}^{i}, x_{1}^{j}} & K_{x_{2}^{i}, x_{2}^{j}} & K_{x_{2}^{i}, x_{3}^{j}} \\
K_{x_{3}^{i}, x_{1}^{j}} & K_{x_{3}^{i}, x_{2}^{j}} & K_{x_{3}^{i}, x_{3}^{j}}
\end{array}\right)
$$

Note that $K^{i j}$ is in general not hermitian, unless $i=j$.

\section{A.11.2 Harrison Interatomic Potential}

One way to calculate the potential energy change in a crystal when one or more atoms aren't on their equilibrium positions is using empirical potentials $U$. One example, specifically useful for zincblende structures, is Harrison interatomic potential [90], which describes the crystal's energy in terms of 2 body $U_{i j}$ and 3 body $U_{i j k}$ interactions as

$$
U=\sum_{i \leq j} U_{i j}+\sum_{i \leq j \leq k} U_{i j k}
$$

Favoring the equilibrium distance between atoms over any stretched configuration, the 2 body interaction $U_{i j}$ characterizes the energy of closest atoms $i$ and $j$ as

$$
U_{i j}=\frac{1}{2} C_{0} \frac{\left(d-d_{0}\right)^{2}}{d_{0}^{2}}
$$

with $d$ and $d_{0}$ the distance and equilibrium distance between the atoms [90]. Assuming that the positions of atom $i$ and $j$ are given by $\left(r_{1}^{i}, r_{2}^{i}, r_{3}^{i}\right)$ and $\left(r_{1}^{j}, r_{2}^{j}, r_{3}^{j}\right)$ respectively, the second derivative of this potential at $d=d_{0}$ is given by

$$
\left.\frac{\partial^{2} U_{i j}}{\partial x_{q}^{\beta} \partial x_{p}^{\alpha}}\right|_{d=d_{0}}=\frac{C_{0}}{d_{0}^{2}} \frac{\partial d}{\partial x_{q}^{\beta}} \frac{\partial d}{\partial x_{p}^{\alpha}}
$$

where $\alpha, \beta=i, j, p, q=1,2,3$ and

$$
\begin{gathered}
\frac{\partial d}{\partial x_{p}^{i}}=-\frac{r_{p}^{j}-r_{p}^{i}}{d_{0}} \\
\frac{\partial d}{\partial x_{p}^{j}}=\frac{r_{p}^{j}-r_{p}^{i}}{d_{0}}
\end{gathered}
$$


The derivatives of $x_{q}^{\beta}$ are the same replacing $p$ by $q$.

On the other hand, favoring the equilibrium angle between bonds, the 3 body interaction $U_{i j k}$ describes the energy of adjacent bonds formed by atoms $i j$ and $i k$ as

$$
U_{i j k}=\frac{1}{2} C_{1}\left(\theta_{i j k}-\theta_{0}\right)
$$

with $\theta_{i j k} \equiv \theta$ and $\theta_{0}$ the angle and equilibrium angle between the bonds [90]. Assuming that the positions of atom $i, j$ and $k$ are given by $\left(r_{1}^{i}, r_{2}^{i}, r_{3}^{i}\right),\left(r_{1}^{j}, r_{2}^{j}, r_{3}^{j}\right)$ and $\left(r_{1}^{k}, r_{2}^{k}, r_{3}^{k}\right)$ respectively and that atom $i$ is the center atom, the second derivative of this potential at $\theta=\theta_{0}$ is given by

$$
\left.\frac{\partial^{2} U_{i j k}}{\partial x_{q}^{\beta} \partial x_{p}^{\alpha}}\right|_{\theta=\theta_{0}}=C_{1} \frac{\partial \theta}{\partial x_{q}^{\beta}} \frac{\partial \theta}{\partial x_{p}^{\alpha}}
$$

where $p, q=1,2,3, \alpha, \beta=i, j, k$ and

$$
\begin{gathered}
\boldsymbol{a}=\left\langle a_{1}, a_{2}, a_{3}\right\rangle=\left\langle r_{1}^{j}-r_{1}^{i}, r_{2}^{j}-r_{2}^{i}, r_{3}^{j}-r_{3}^{i}\right\rangle \\
\boldsymbol{b}=\left\langle b_{1}, b_{2}, b_{3}\right\rangle=\left\langle r_{1}^{k}-r_{1}^{i}, r_{2}^{k}-r_{2}^{i}, r_{3}^{k}-r_{3}^{i}\right\rangle \\
\frac{\partial \theta}{\partial x_{p}^{i}}=\frac{1}{\sqrt{1-\left(\frac{\boldsymbol{a} \cdot \boldsymbol{b}}{a b}\right)^{2}}}\left[\frac{b_{p}+a_{p}}{a b}-\frac{\boldsymbol{a} \cdot \boldsymbol{b}}{(a b)^{2}}\left(\frac{b}{a} a_{p}+\frac{a}{b} b_{p}\right)\right] \\
\frac{\partial \theta}{\partial x_{p}^{j}}=\frac{-1}{\sqrt{1-\left(\frac{\boldsymbol{a} \cdot \boldsymbol{b}}{a b}\right)^{2}}}\left[\frac{b_{p}}{a b}-\frac{\boldsymbol{a} \cdot \boldsymbol{b}}{(a b)^{2}} \frac{b}{a} a_{p}\right] \\
\frac{\partial \theta}{\partial x_{p}^{k}}=\frac{-1}{\sqrt{1-\left(\frac{\boldsymbol{a} \cdot \boldsymbol{b}}{a b}\right)^{2}}}\left[\frac{a_{p}}{a b}-\frac{\boldsymbol{a} \cdot \boldsymbol{b}}{(a b)^{2}} \frac{a}{b} b_{p}\right]
\end{gathered}
$$

The derivatives of $x_{q}^{\beta}$ are the same replacing $p$ by $q$.

\section{A.11.3 Tensor Force Constants for Si}

Silicon is a FCC crystal with lattice constant $a=5.43 \AA$. It has 2 atoms per unit cell, each one with mass $m=4.664 \times 10^{-26} \mathrm{~kg}$, joined by the vector $a / 4(-\hat{\boldsymbol{x}}+\hat{\boldsymbol{y}}+\hat{\boldsymbol{z}})$ and separated by $d_{0}=\sqrt{3} a / 4$. These atoms will be referred as site A and site B. The potential energy $U$ of $\mathrm{Si}$ is found using Harrison interatomic potential with $C_{0}=49.1 \mathrm{eV}$ and $C_{1}=1.07 \mathrm{eV}$ [90]. Since the 2 and 3 body potentials describe the stretching energy of closest atoms and bending energy of adjacent bonds, only the tensor force constants relating an atom with up to its second neighbors are different from 0 . Therefore, for every atom in the crystal its interactions with the 16 nearest neighboring atoms and with itself need to be considered. For atoms A and B in the unit cell 
$n_{0}$, the neighbors are numbered according to Figure A.9 and their relative positions with respect to A and B and the site they occupy are listed in Table A.1.

\begin{tabular}{|c|c|c|c|c|c|}
\hline \multicolumn{3}{|c|}{ Neighbors of A } & \multicolumn{3}{|c|}{ Neighbors of B } \\
\hline$i$ & $\boldsymbol{r}_{i}-\boldsymbol{r}_{A}$ & Site & $i$ & $\boldsymbol{r}_{i}-\boldsymbol{r}_{B}$ & Site \\
\hline $\mathrm{A}$ & $\langle 0,0,0\rangle$ & $\mathrm{A}$ & $\mathrm{B}$ & $\langle 0,0,0\rangle$ & $\mathrm{B}$ \\
\hline 1 & $a / 4\langle-1,1,1\rangle$ & $\mathrm{B}$ & 1 & $a / 4\langle-1,1,-1\rangle$ & $\mathrm{A}$ \\
\hline 2 & $a / 4\langle-2,0,2\rangle$ & $\mathrm{A}$ & 2 & $a / 4\langle-2,0,-2\rangle$ & $\mathrm{B}$ \\
\hline 3 & $a / 4\langle-2,2,0\rangle$ & $\mathrm{A}$ & 3 & $a / 4\langle-2,2,0\rangle$ & $\mathrm{B}$ \\
\hline 4 & $a / 4\langle 0,2,2\rangle$ & $\mathrm{A}$ & 4 & $a / 4\langle 0,2,-2\rangle$ & $\mathrm{B}$ \\
\hline 5 & $a / 4\langle 1,1,-1\rangle$ & $\mathrm{B}$ & 5 & $a / 4\langle 1,1,1\rangle$ & $\mathrm{A}$ \\
\hline 6 & $a / 4\langle 0,2,-2\rangle$ & $\mathrm{A}$ & 6 & $a / 4\langle 0,2,2\rangle$ & $\mathrm{B}$ \\
\hline 7 & $a / 4\langle 2,2,0\rangle$ & $\mathrm{A}$ & 7 & $a / 4\langle 2,2,0\rangle$ & $\mathrm{B}$ \\
\hline 8 & $a / 4\langle 2,0,-2\rangle$ & $\mathrm{A}$ & 8 & $a / 4\langle 2,0,2\rangle$ & $\mathrm{B}$ \\
\hline 9 & $a / 4\langle 1,-1,1\rangle$ & $\mathrm{B}$ & 9 & $a / 4\langle 1,-1,-1\rangle$ & $\mathrm{A}$ \\
\hline 10 & $a / 4\langle 2,0,2\rangle$ & $\mathrm{A}$ & 10 & $a / 4\langle 2,0,-2\rangle$ & $\mathrm{B}$ \\
\hline 11 & $a / 4\langle 2,-2,0\rangle$ & $\mathrm{A}$ & 11 & $a / 4\langle 2,-2,0\rangle$ & $\mathrm{B}$ \\
\hline 12 & $a / 4\langle 0,-2,2\rangle$ & $\mathrm{A}$ & 12 & $a / 4\langle 0,-2,-2\rangle$ & $\mathrm{B}$ \\
\hline 13 & $a / 4\langle-1,-1,-1\rangle$ & $\mathrm{B}$ & 13 & $a / 4\langle-1,-1,1\rangle$ & $\mathrm{A}$ \\
\hline 14 & $a / 4\langle 0,-2,-2\rangle$ & $\mathrm{A}$ & 14 & $a / 4\langle 0,-2,2\rangle$ & $\mathrm{B}$ \\
\hline 15 & $a / 4\langle-2,-2,0\rangle$ & $\mathrm{A}$ & 15 & $a / 4\langle-2,-2,0\rangle$ & $\mathrm{B}$ \\
\hline 16 & $a / 4\langle-2,0,-2\rangle$ & $\mathrm{A}$ & 16 & $a / 4\langle-2,0,2\rangle$ & $\mathrm{B}$ \\
\hline
\end{tabular}

Table A.1: Labeling of neighbors of A and B, vectors from A (B) to the neighbor and site they occupy.
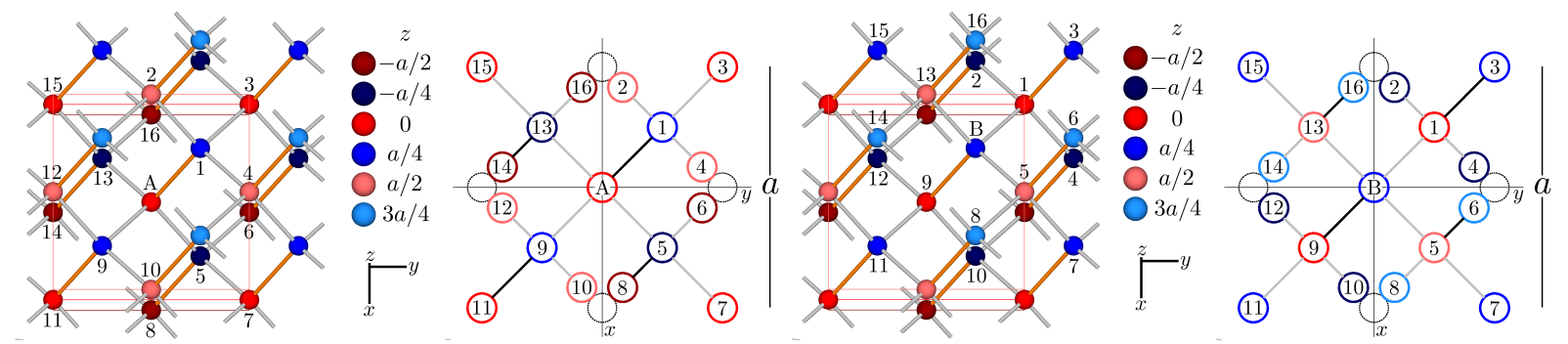

Figure A.9: Numbering of the 16 nearest neighbors of site A and B. The reddish atoms occupy site A, the bluish atoms occupy site B. The lighter the color the larger the $z$ component of the atom. The orange bonds joined atoms in the same unit cell up Left) Neighbors of A up right) $x y$ view of the neighbors of A. Atom 4 and 6 are displaced from their equilibrium position (black circumference) as a visualization tool. The projected view only will show 4 since 6 is exactly under 4 . Similarly with 8,10; 12,14 and 16,2 down Left) Neighbors of B down right $x y$ view of the neighbors of $\mathrm{B}$. 
The tensor force constant $K^{A A}$ is obtained using equations A.80, A.81, A.82 and A.83 and the potential energy

$$
\begin{aligned}
U & =U_{A, 1}+U_{A, 5}+U_{A, 9}+U_{A, 13}+U_{A, 1,5}+U_{A, 1,9} \\
& +U_{A, 1,13}+U_{A, 5,9}+U_{A, 5,13}+U_{A, 9,13}+U_{1, A, 2}+U_{1, A, 3} \\
& +U_{1, A, 4}+U_{5, A, 6}+U_{5, A, 7}+U_{5, A, 8}+U_{9, A, 10}+U_{9, A, 11} \\
& +U_{9, A, 12}+U_{13, A, 14}+U_{13, A, 15}+U_{13, A, 16}
\end{aligned}
$$

Because of symmetry, if atom $\mathrm{A}$ is moved in the $\hat{\boldsymbol{x}}_{1}^{A}$ direction only a restoring force in the same direction is expected, then $K^{A A}$ is diagonal and is given by

$$
K^{A A}=\left(\begin{array}{ccc}
\zeta_{0} & 0 & 0 \\
0 & \zeta_{0} & 0 \\
0 & 0 & \zeta_{0}
\end{array}\right)[N / m]
$$

with $\zeta_{0}=218.645231966192781$

The tensor force constant $K^{A 1}$ is obtained using equations A.80, A.81, A.82 and A.83 and the potential energy

$$
U=U_{A, 1}+U_{A, 1,5}+U_{A, 1,9}+U_{A, 1,13}+U_{1, A, 2}+U_{1, A, 3}+U_{1, A, 4}
$$

Note that the interactions with non vanishing second derivatives are those depending on degrees of freedom of $A$ and 1 at the same time. Calculating the derivatives $K^{A 1}$ results to be

$$
K^{A 1}=\left(\begin{array}{ccc}
-\zeta_{1} & \zeta_{2} & \zeta_{2} \\
\zeta_{2} & -\zeta_{1} & -\zeta_{2} \\
\zeta_{2} & -\zeta_{2} & -\zeta_{1}
\end{array}\right)[N / m]
$$

with $\zeta_{1}=55.694840409836885$ and $\zeta_{2}=43.292451390372683$

Because of crystal symmetry, $K^{B 1}, K^{A 5}, K^{B 5}, K^{A 9}, K^{B 9}, K^{A 13}$ and $K^{B 13}$ can be found from $K^{A 1}$. For instance, if the crystal is inverted through the position of $\mathrm{A}$ the crystal at $\mathrm{B}$ is gotten, therefore $K^{B 9}$ is the inverted version of $K^{A 1}$ (Figure A.9). That is, if the inversion of a vector through A is represented by the matrix $\operatorname{Inv}(A)$ then

$$
K^{B 9}=\operatorname{Inv}(A) K^{A 1} \operatorname{Inv}(A)^{\dagger}=K^{A 1}
$$


since $\operatorname{Inv}(A)=-I$. Also, representing the rotation of a vector around the $z$ axis by

$$
R_{z}(\phi)=\left(\begin{array}{ccc}
\cos \phi & -\sin \phi & 0 \\
\sin \phi & \cos \phi & 0 \\
0 & 0 & 1
\end{array}\right)
$$

and using the fact that rotating the crystal about a $z$ axis at position A by $\pi$ gives the same crystal (Figure A.9) the relashionship

$$
K^{A 9}=R_{z}(\pi) K^{A 1} R_{z}(\pi)^{\dagger}
$$

follows. By inversion of $K^{A 9}, K^{B 1}$ is also found to be

$$
K^{B 1}=K^{A 9}
$$

Similarly, using other rotation and inversion symmetries

$$
\begin{gathered}
K^{B 13}=R_{z}(\pi / 2) K^{A 1} R_{z}(\pi / 2)^{\dagger}=K^{A 5} \\
K^{B 5}=R_{z}(-\pi / 2) K^{A 1} R_{z}(-\pi / 2)^{\dagger}=K^{A 13}
\end{gathered}
$$

$K^{A 2}$ is obtained using the potential $U=U_{1 A 2}$ as

$$
K^{A 2}=\left(\begin{array}{ccc}
-\zeta_{3} & \zeta_{4} & \zeta_{3} \\
-\zeta_{4} & \zeta_{5} & \zeta_{4} \\
\zeta_{3} & -\zeta_{4} & -\zeta_{3}
\end{array}\right)[N / m]
$$

with $\zeta_{3}=0.516766209144342, \zeta_{4}=1.033532418288683$ and $\zeta_{5}=2.067064836577367$. Using the symmetry of the crystal $K^{B 2}, K^{A 4}, K^{B 4}, K^{A 6}, K^{B 6}, K^{A 8}, K^{B 8}, K^{A 10}, K^{B 10}, K^{A 12}, K^{B 12}, K^{A 14}, K^{B 14}, K^{A 16}$ and $K^{B 16}$ are obtained

$$
\begin{gathered}
K^{A 2}=K^{B 10} \\
K^{A 10}=R_{z}(\pi) K^{A 2} R_{z}(\pi)^{\dagger}=K^{B 2} \\
K^{B 14}=R_{z}(\pi / 2) K^{A 2} R_{z}(\pi / 2)^{\dagger}=K^{A 6} \\
K^{B 6}=R_{z}(-\pi / 2) K^{A 2} R_{z}(-\pi / 2)^{\dagger}=K^{A 14}
\end{gathered}
$$


Also, representing the reflexion of a vector though the plane $y=-x$ by

$$
\begin{aligned}
& \operatorname{Ref}=\left(\begin{array}{ccc}
0 & -1 & 0 \\
-1 & 0 & 0 \\
0 & 0 & 1
\end{array}\right) \\
& K^{A 4}=\operatorname{RefK^{A2}} \operatorname{Ref}^{\dagger}
\end{aligned}
$$

and from the symmetry of the crystal follows

$$
\begin{gathered}
K^{A 4}=K^{B 12} \\
K^{A 12}=R_{z}(\pi) K^{A 4} R_{z}(\pi)^{\dagger}=K^{B 4} \\
K^{B 16}=R_{z}(\pi / 2) K^{A 4} R_{z}(\pi / 2)^{\dagger}=K^{A 8} \\
K^{B 8}=R_{z}(-\pi / 2) K^{A 4} R_{z}(-\pi / 2)^{\dagger}=K^{A 16}
\end{gathered}
$$

$K^{A 3}$ is obtained using the potential $U=U_{1 A 3}$ as

$$
K^{A 3}=\left(\begin{array}{ccc}
-\zeta_{3} & \zeta_{3} & \zeta_{4} \\
\zeta_{3} & -\zeta_{3} & -\zeta_{4} \\
-\zeta_{4} & \zeta_{4} & \zeta_{5}
\end{array}\right)[N / m]
$$

From the symmetry of the crystal $K^{B 3}, K^{A 7}, K^{B 7}, K^{A 11}, K^{B 11}, K^{A 15}$ and $K^{B 15}$ are given by

$$
\begin{gathered}
K^{A 3}=K^{B 11} \\
K^{A 11}=R_{z}(\pi) K^{A 3} R_{z}(\pi)^{\dagger}=K^{B 3} \\
K^{B 15}=R_{z}(\pi / 2) K^{A 3} R_{z}(\pi / 2)^{\dagger}=K^{A 7} \\
K^{B 7}=R_{z}(-\pi / 2) K^{A 3} R_{z}(-\pi / 2)^{\dagger}=K^{A 15}
\end{gathered}
$$

\section{A.11.4 Force Constants Relating Units Cells}

With the tensor force constants relating individual atoms, the 13 force constants relating a unit cell with itself and with its 12 nearest neighbors are built using Figure A.9 and A.10. 


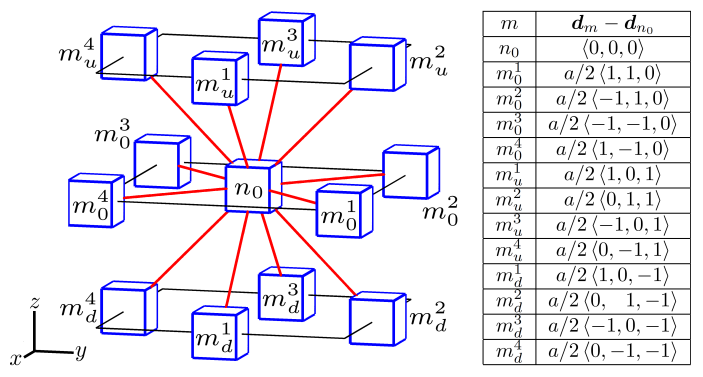

Figure A.10: Unit cell $n_{0}$ with its 12 neighboring unit cells. Red bars represent coupling between unit cells. $\mathrm{d}$ and $\mathrm{u}$ stand for down and up. Table on the right shows the position vectors of each unit cell relative to the center unit cell.

$$
\begin{aligned}
& K^{n_{0}}=\left[\begin{array}{cc}
K^{A A} & K^{A 1} \\
K^{B 9} & K^{B B}
\end{array}\right] \quad K^{m_{0}^{1}}=\left[\begin{array}{cc}
K^{A 7} & 0 \\
0 & K^{B 7}
\end{array}\right] \\
& K^{m_{0}^{2}}=\left[\begin{array}{cc}
K^{A 3} & 0 \\
K^{B 1} & K^{B 3}
\end{array}\right] \quad K^{m_{0}^{3}}=\left[\begin{array}{cc}
K^{A 15} & 0 \\
0 & K^{B 15}
\end{array}\right] \\
& K^{m_{0}^{4}}\left[\begin{array}{cc}
K^{A 11} & K^{A 9} \\
0 & K^{B 11}
\end{array}\right] \quad K^{m_{u}^{1}}=\left[\begin{array}{cc}
K^{A 10} & 0 \\
0 & K^{B 8}
\end{array}\right] \\
& K^{m_{u}^{2}}=\left[\begin{array}{cc}
K^{A 4} & 0 \\
K^{B 5} & K^{B 6}
\end{array}\right] \quad K^{m_{u}^{3}}=\left[\begin{array}{cc}
K^{A 2} & 0 \\
K^{B 13} & K^{B 16}
\end{array}\right] \\
& K^{m_{u}^{4}}=\left[\begin{array}{cc}
K^{A 12} & 0 \\
0 & K^{B 14}
\end{array}\right] \quad K^{m_{d}^{1}}=\left[\begin{array}{cc}
K^{A 8} & K^{A 5} \\
0 & K^{B 10}
\end{array}\right] \\
& K^{m_{d}^{2}}=\left[\begin{array}{cc}
K^{A 6} & 0 \\
0 & K^{B 4}
\end{array}\right] \quad K^{m_{d}^{3}}=\left[\begin{array}{cc}
K^{A 16} & 0 \\
0 & K^{B 2}
\end{array}\right] \\
& K^{m_{d}^{4}}=\left[\begin{array}{cc}
K^{A 14} & K^{A 13} \\
0 & K^{B 12}
\end{array}\right]
\end{aligned}
$$

\section{A.11.5 Dispersion Relation $\omega$ vs $k$}

To find the dispersion relationship, plane wave solutions $\boldsymbol{u}=\boldsymbol{u}_{0} e^{i \boldsymbol{k} \cdot \boldsymbol{d}_{n}}$ are assumed on Newton's time independent equation. After a similar process to the one in subsection A.4.1, this equation is changed to $\boldsymbol{k}$ space

$$
\omega^{2} \mathbb{M} \boldsymbol{u}_{0}=\mathbb{K}(\boldsymbol{k}) \boldsymbol{u}_{0}
$$


where $\mathbb{M}$ and $\mathbb{K}$ are square matrices of size \# atoms per unit cell $\times$ degrees of freedom per atom $d_{f}$. For instance, $2 \cdot 3=6$ for Silicon. $\mathbb{M}$ is a diagonal matrix containing the mass of each unit cell atom repeated $d_{f}$ times and $\mathbb{K}$ is given by

$$
\mathbb{K}(\boldsymbol{k})=\sum_{m} K^{m} \exp \left(i \boldsymbol{k} \cdot\left(\boldsymbol{d}_{m}-\boldsymbol{d}_{n_{0}}\right)\right)
$$

where $m$ refers to the neighbor unit cells including itself and $\boldsymbol{d}_{m}$ refers to the position of the unit cell. For the particular case of Silicon and based on the notation introduced in Figure A.10 $\mathbb{K}(\boldsymbol{k})$ is given by

$$
\begin{aligned}
& \mathbb{K}(\boldsymbol{k})=K^{n_{0}}+K^{m_{0}^{1}} \exp \left[i \frac{a}{2}\left(k_{x}+k_{y}\right)\right] \\
& +K^{m_{0}^{2}} \exp \left[i \frac{a}{2}\left(-k_{x}+k_{y}\right)\right]+K^{m_{0}^{3}} \exp \left[i \frac{a}{2}\left(-k_{x}-k_{y}\right)\right] \\
& +K^{m_{0}^{4}} \exp \left[i \frac{a}{2}\left(k_{x}-k_{y}\right)\right]+K^{m_{u}^{1}} \exp \left[i \frac{a}{2}\left(k_{x}+k_{z}\right)\right] \\
& +K^{m_{u}^{2}} \exp \left[i \frac{a}{2}\left(k_{y}+k_{z}\right)\right]+K^{m_{u}^{3}} \exp \left[i \frac{a}{2}\left(-k_{x}+k_{z}\right)\right] \\
& +K^{m_{u}^{4}} \exp \left[i \frac{a}{2}\left(-k_{y}+k_{z}\right)\right]+K^{m_{d}^{1}} \exp \left[i \frac{a}{2}\left(k_{x}-k_{z}\right)\right] \\
& +K^{m_{d}^{2}} \exp \left[i \frac{a}{2}\left(k_{y}-k_{z}\right)\right]+K^{m_{d}^{3}} \exp \left[i \frac{a}{2}\left(-k_{x}-k_{z}\right)\right] \\
& +K^{m_{d}^{4}} \exp \left[i \frac{a}{2}\left(-k_{y}-k_{z}\right)\right]
\end{aligned}
$$

Now, finding the dispersion relation along a particular direction in $\boldsymbol{k}$ space becomes a matter of defining a line in $\boldsymbol{k}$ space and plot the square root of the eigenvalues of $\mathbb{M}^{-1} \mathbb{K}(\boldsymbol{k})$ for each $\boldsymbol{k}$ in the line.

$$
\omega^{2} \boldsymbol{u}_{0}=\mathbb{M}^{-1} \mathbb{K}(\boldsymbol{k}) \boldsymbol{u}_{0}
$$

This procedure generates six bands, three acoustic and three optical. Silicon dispersion relation using the force constants obtained with Harrison interatomic potential is shown in Figure A.11
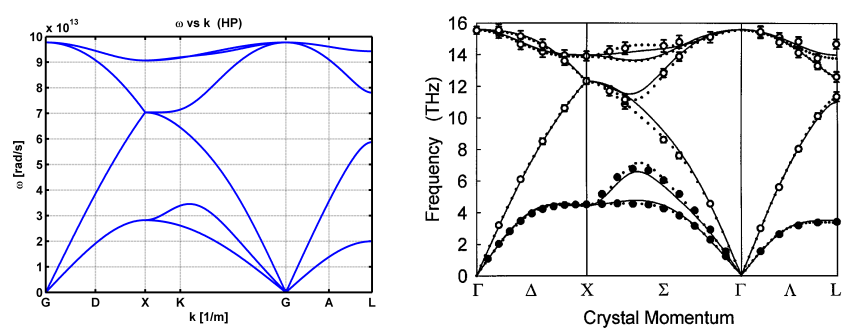

Figure A.11: Si dispersion relation Left using the force constants obtained with Harrison interatomic potential Right Experimental data by [8] 


\section{A.11.6 Density of states}

A "numerical count of states" referred as binning can be found by calculating the eigen frequencies for some equally space set of $\boldsymbol{k}$ points inside the first Brillouin zone and then counting how many of them lie in a particular frequency interval. From that an estimate of the density of states can be found. Indeed, the states inside $\Delta \omega$ are

$$
N(\omega) \Delta \omega=\sum_{\substack{\boldsymbol{k} \in B Z \\ \omega<\omega(\boldsymbol{k}) \\ \omega(\boldsymbol{k})<\omega+\Delta \omega}} 1=\frac{1}{\Delta k_{x} \Delta k_{y} \Delta k_{z}} \int_{R} d k_{x} d k_{y} d k_{z}
$$

For the second equality, a large size material, periodic boundary conditions and $R=\{\boldsymbol{k} \mid \omega<\omega(\boldsymbol{k})<\omega+\Delta \omega\}$ were assumed. Then the integral is approximated numerically as

$$
N(\omega) \Delta \omega=\frac{V}{8 \pi^{3}}\left(\begin{array}{c}
\text { Numerical } \\
\text { count of states }
\end{array}\right) \Delta k_{x} \Delta k_{y} \Delta k_{z}
$$

and the Density Of State per unit volume is

$$
\operatorname{DOS}(\omega)=\frac{1}{8 \pi^{3}}\left(\begin{array}{c}
\text { Numerical } \\
\text { count of states }
\end{array}\right) \frac{\Delta k_{x} \Delta k_{y} \Delta k_{z}}{\Delta \omega}
$$

The DOS for Silicon is shown in Figure A.12

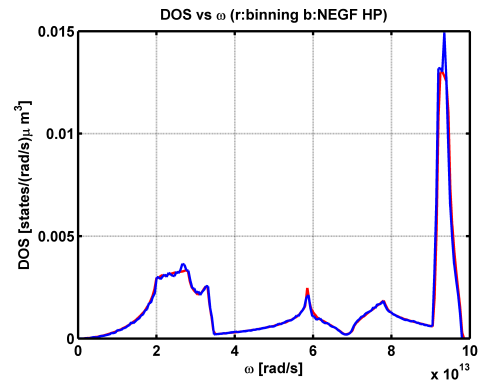

Figure A.12: Density of States for Silicon using the force constants obtained with Harrison interatomic potential. Blue) calculation with NEGF. Red) calculation by binning.

From the device Green's function $G$ or from the spectral function $A$ the DOS in the device can also be found (equation A.33)

$$
D O S_{d}=\operatorname{Tr}\left(\frac{i \omega}{\pi} M^{1 / 2}\left(G-G^{\dagger}\right) M^{1 / 2}\right)
$$


Note that since $M=m I$ (I is the identity matrix), the $D O S_{d}$ can be simplified to

$$
D O S_{d}=\operatorname{Tr}\left(\frac{\omega m}{\pi} A\right)
$$

To obtain the density of states per unit volume, this quantity must be normalized by the volume of the device. For the particular simulation in this paper, the device is a primitive unit cell of Silicon with 2 atoms and a volume of $a^{3} / 4$. Then the DOS per unit volume is given by (Figure A.12)

$$
D O S=\frac{4}{a^{3}} D O S_{d}
$$




\section{Appendix B}

\section{Anharmonicity}

This chapter is meant as a help to follow the reference [28], where the process to included phonon-phonon interactions in NEGF is develop, with the different notation we have used over the document.

\section{B.1 Quantum Harmonic Oscillator: 1D crystal}

Imagine we wave a 1D chain of atoms linked by springs that is periodic after $N$ atoms (Fig. B.1). Where $r_{n}$ is the position, $x_{n}$ is the deviation from equilibrium and $m$ is the mass of atom $n$ and $\kappa$ is the spring constant of each spring. The total energy of the system or classical Hamiltonian is given by the sum of the kinetic

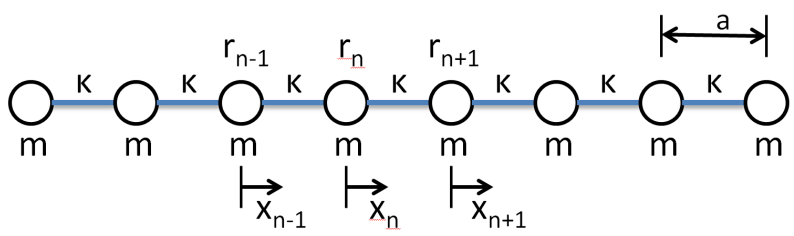

Figure B.1: 1D crystal

energy of every atom plus the potential energy stored in the springs for every pair of atoms. That is

$$
H=\sum_{n=0}^{N-1} \frac{1}{2} m \dot{x}_{n}^{2}+\sum_{n=0}^{N-1} \frac{1}{2} \kappa\left(x_{n+1}-x_{n}\right)^{2}
$$

This Hamiltonian is not diagonal, in other words we have a bunch of couple oscillators (each atom is an oscillator and it is couple with the movement of its two neighbors). Since the system is translational invariant or periodic, we can decouple the oscillators by performing a Fourier transform. This procedure is referred as splitting the system in normal modes, which each normal mode corresponding to a Fourier component. So we 
define the following transform

$$
x_{n}=\frac{1}{\sqrt{N}} \sum_{k=0}^{N-1} \tilde{x}_{k} e^{i k r_{n}}
$$

Replacing this transformation for $x_{n}$ into the Hamiltonian and using the identity

$$
\sum_{n=0}^{N-1} e^{i k x_{n}}=N \delta_{k, 0}
$$

we get

$$
H=\sum_{k=0}^{N-1} \frac{1}{2} \frac{\tilde{p}_{k} \tilde{p}_{-k}}{m}+\frac{1}{2} m \omega_{k}^{2} \tilde{x}_{k} \tilde{x}_{-k}
$$

with

$$
\omega_{k}^{2}=\frac{2 \kappa}{m}(1-\cos k a)=\frac{4 \kappa}{m} \sin ^{2} \frac{k a}{2}
$$

and

$$
p_{n}=m \dot{x}_{n} \quad \tilde{p}_{k}=m \dot{\tilde{x}}_{k}
$$

At this points it looks like we have $2 N$ degrees of freedom. Two for each of $k$ since $\tilde{x}_{k}$ is complex. Those degrees of freedom are not actually independent because we need to guarantee that $x_{n}$ is real, which implies that

$$
\tilde{x}_{k}^{*}=\tilde{x}_{-k}
$$

This result follows from comparing $x_{k}$ with its complex conjugate:

$$
\begin{aligned}
\tilde{x}_{k} & =\frac{1}{\sqrt{N}} \sum_{n=0}^{N-1} \tilde{x}_{n} e^{-i k r_{n}} \\
\tilde{x}_{k}^{*} & =\frac{1}{\sqrt{N}} \sum_{n=0}^{N-1} \tilde{x}_{n} e^{i k r_{n}} \\
& =\frac{1}{\sqrt{N}} \sum_{n=0}^{N-1} \tilde{x}_{n} e^{-i(-k) r_{n}} \\
& =\tilde{x}_{-k}
\end{aligned}
$$


Now we promote the classical variables to quantum operators and state the usual commutation rules between canonical variables

$$
\begin{aligned}
\tilde{x}_{k} & \rightarrow \tilde{X}_{k} \\
\tilde{x}_{k}^{*} & \rightarrow \tilde{X}_{k}^{\dagger} \\
\tilde{p}_{k} & \rightarrow \tilde{P}_{k} \\
\tilde{p}_{k}^{*} & \rightarrow \tilde{P}_{k}^{\dagger} \\
\tilde{X}_{k}^{\dagger} & =\tilde{X}_{-k} \\
\tilde{P}_{k}^{\dagger} & =\tilde{P}_{-k}
\end{aligned}
$$

Then the Hamiltonian becomes

$$
H=\sum_{k=0}^{N-1} \frac{1}{2} \frac{\tilde{P}_{k} \tilde{P}_{-k}}{m}+\frac{1}{2} m \omega_{k}^{2} \tilde{X}_{k} \tilde{X}_{-k}
$$

For each $k$, this Hamiltonian is very similar to the harmonic oscillator but with a mix between $+k$ and $-k$. So we use operators similar to the creation and annihilation operators to further simplify the Hamiltonian.

$$
\begin{aligned}
a_{k} & =\sqrt{\frac{m \omega_{k}}{2 \hbar}} X_{k}+i \sqrt{\frac{1}{2 m \omega_{k} \hbar}} P_{-k} \\
a_{k}^{\dagger} & =\sqrt{\frac{m \omega_{k}}{2 \hbar}} X_{-k}-i \sqrt{\frac{1}{2 m \omega_{k} \hbar}} P_{k} \\
\tilde{X}_{k} & =\sqrt{\frac{\hbar}{2 m \omega_{k}}}\left(a_{k}+a_{-k}^{\dagger}\right) \\
P_{k} & =-i \sqrt{\frac{\hbar m \omega_{k}}{2}}\left(a_{k}-a_{-k}^{\dagger}\right)
\end{aligned}
$$

Note that

$$
\begin{aligned}
\tilde{X}_{k} \tilde{X}_{-k} & =\frac{\hbar}{2 m \omega_{k}}\left(a_{k} a_{-k}+a_{k} a_{k}^{\dagger}+a_{-k}^{\dagger} a_{-k}+a_{-k}^{\dagger} a_{k}^{\dagger}\right) \\
\tilde{P}_{k} \tilde{P}_{-k} & =\frac{\hbar m \omega_{k}}{2}\left(-a_{k} a_{-k}+a_{k} a_{k}^{\dagger}+a_{-k}^{\dagger} a_{-k}-a_{-k}^{\dagger} a_{k}^{\dagger}\right)
\end{aligned}
$$

so replacing into the Hamiltonian we get

$$
H=\sum_{k=0}^{N-1} \frac{1}{2} \hbar \omega_{k}\left(a_{k} a_{k}^{\dagger}+a_{-k}^{\dagger} a_{-k}\right)
$$


Now we reorganize the sum and used the commutation relation $\left[a_{k}, a_{k}^{\dagger}\right]=a_{k} a_{k}^{\dagger}-a_{k}^{\dagger} a_{k}=1$

$$
\begin{aligned}
H & =\sum_{k=0}^{N-1} \frac{1}{2} \hbar \omega_{k}\left(a_{k} a_{k}^{\dagger}+a_{k}^{\dagger} a_{k}\right) \\
H & =\sum_{k=0}^{N-1} \frac{1}{2} \hbar \omega_{k}\left(2 a_{k}^{\dagger} a_{k}+1\right) \\
H & =\sum_{k=0}^{N-1} \hbar \omega_{k}\left(a_{k}^{\dagger} a_{k}+\frac{1}{2}\right)
\end{aligned}
$$

and

$$
\begin{aligned}
& X_{n}=\frac{1}{\sqrt{N}} \sum_{k=0}^{N-1} \tilde{X}_{k} e^{i k r_{n}} \\
& X_{n}=\frac{1}{\sqrt{N}} \sum_{k=0}^{N-1} \sqrt{\frac{\hbar}{2 m \omega_{k}}}\left(a_{k}+a_{-k}^{\dagger}\right) e^{i k r_{n}} \\
& X_{n}=\frac{1}{\sqrt{N}} \sum_{k=0}^{N-1} \sqrt{\frac{\hbar}{2 m \omega_{k}}}\left(a_{k} e^{i k r_{n}}+a_{-k}^{\dagger} e^{i k r_{n}}\right) \\
& X_{n}=\frac{1}{\sqrt{N}} \sum_{k=0}^{N-1} \sqrt{\frac{\hbar}{2 m \omega_{k}}}\left(a_{k} e^{i k r_{n}}+a_{k}^{\dagger} e^{-i k r_{n}}\right)
\end{aligned}
$$

letting the creation and annihilation operator evolve in time we get

$$
x_{n}(t)=\sum_{m} \sqrt{\frac{\hbar}{2 \omega_{m}}}\left[e_{n}^{m} a_{m} e^{-i \omega_{m} t}+\left(e_{n}^{m}\right)^{*} a_{m}^{\dagger} e^{i \omega_{m} t}\right]
$$

Note that for $x_{n}$ to be real $a_{-m}=a_{m}^{\dagger}$ and $e_{n}^{-m}=\left(e_{n}^{m}\right)^{*}$

\section{B.2 Green's Functions}

$$
\begin{aligned}
& x_{n_{1}}\left(t_{1}\right)=\sum_{m_{1}} \sqrt{\frac{\hbar}{2 \omega_{m_{1}}}}\left[e_{n_{1}}^{m_{1}} a_{m_{1}} e^{-i \omega_{m_{1}} t_{1}}+\left(e_{n_{1}}^{m_{1}}\right)^{*} a_{m_{1}}^{\dagger} e^{i \omega_{m_{1}} t_{1}}\right] \\
& x_{n_{2}}\left(t_{2}\right)=\sum_{m_{2}} \sqrt{\frac{\hbar}{2 \omega_{m_{2}}}}\left[e_{n_{2}}^{m_{2}} a_{m_{2}} e^{-i \omega_{m_{2}} t_{2}}+\left(e_{n_{2}}^{m_{2}}\right)^{*} a_{m_{2}}^{\dagger} e^{i \omega_{m_{2}} t_{2}}\right]
\end{aligned}
$$


Let me start by calculating the equilibrium green's function.

$$
\begin{aligned}
{\left[G_{r_{i}}^{n}\left(t_{1}, t_{2}\right)\right]_{n_{1}, n_{2}} } & =\left[i D_{0}^{<}\left(t_{1}, t_{2}\right)\right]_{n_{1}, n_{2}} \\
{\left[G_{r_{i}}^{n}(\tau)\right]_{n_{1}, n_{2}} } & =\left[i D_{0}^{<}\left(t_{1}-t_{2}\right)\right]_{n_{1} n_{2}}=\left[i D_{0}^{<}(\tau)\right]_{n_{1} n_{2}} \\
& =\frac{1}{\hbar}\left\langle x_{n_{2}}\left(t_{2}\right) x_{n_{1}}\left(t_{1}\right)\right\rangle \\
& =\sum_{m_{1} m_{2}} \frac{1}{2 \sqrt{\omega_{m_{1}} \omega_{m_{2}}}}\left[\left\langle a_{m_{2}} a_{m_{1}}^{\dagger}\right\rangle e_{n_{2}}^{m_{2}}\left(e_{n_{1}}^{m_{1}}\right)^{*} e^{i\left(\omega_{m_{1}} t_{1}-\omega_{m_{2}} t_{2}\right)}+\left\langle a_{m_{2}}^{\dagger} a_{m_{1}}\right\rangle\left(e_{n_{2}}^{m_{2}}\right)^{*} e_{n_{1}}^{m_{1}} e^{i\left(\omega_{m_{2}} t_{2}-\omega_{m_{1}} t_{1}\right)}\right] \\
& =\sum_{m_{1} m_{2}} \frac{1}{2 \sqrt{\omega_{m_{1}} \omega_{m_{2}}}}\left[\left(N_{m_{1}}+1\right) \delta_{m_{1}, m_{2}} e_{n_{2}}^{m_{2}}\left(e_{n_{1}}^{m_{1}}\right)^{*} e^{i\left(\omega_{m_{1}} t_{1}-\omega_{m_{2}} t_{2}\right)}+N_{m_{1}} \delta_{m_{1}, m_{2}}\left(e_{n_{2}}^{m_{2}}\right)^{*} e_{n_{1}}^{m_{1}} e^{i\left(\omega_{m_{2}} t_{2}-\omega_{m_{1}} t_{1}\right)}\right] \\
& =\sum_{m} \frac{1}{2 \omega_{m}}\left[\left(N_{m}+1\right) e_{n_{2}}^{m}\left(e_{n_{1}}^{m}\right)^{*} e^{i \omega_{m}\left(t_{1}-t_{2}\right)}+N_{m}\left(e_{n_{2}}^{m}\right)^{*} e_{n_{1}}^{m} e^{i \omega_{m}\left(t_{2}-t_{1}\right)}\right] \\
& =\sum_{m} \frac{1}{2 \omega_{m}}\left[\left(N_{-m}+1\right) e_{n_{2}}^{-m}\left(e_{n_{1}}^{-m}\right)^{*} e^{i \omega_{-m}\left(t_{1}-t_{2}\right)}+N_{m}\left(e_{n_{2}}^{m}\right)^{*} e_{n_{1}}^{m} e^{i \omega_{m}\left(t_{2}-t_{1}\right)}\right] \\
& =\sum_{m} \frac{1}{2 \omega_{m}}\left[\left(N_{-m}+1\right)\left(e_{n_{2}}^{m}\right)^{*} e_{n_{1}}^{m} e^{i \omega_{m} \tau}+N_{m}\left(e_{n_{2}}^{m}\right)^{*} e_{n_{1}}^{m} e^{-i \omega_{m} \tau}\right] \\
& =\sum_{m} \frac{1}{2 \omega_{m}}\left(e_{n_{2}}^{m}\right)^{*} e_{n_{1}}^{m}\left[\left(N_{-m}+1\right) e^{i \omega_{m} \tau}+N_{m} e^{-i \omega_{m} \tau}\right]
\end{aligned}
$$

Fourier transforming the expression with

$$
f(\omega)=\int d \tau f(\tau) e^{i \omega \tau}
$$

using

$$
\begin{gathered}
F\left\{e^{i \omega_{m} t}\right\}=2 \pi \delta\left(\omega+\omega_{m}\right), \\
2 \omega_{m} \delta\left(\omega^{2}-\omega_{m}^{2}\right) \theta(\omega)=\delta\left(\omega-\omega_{m}\right) \quad 2 \omega_{m} \delta\left(\omega^{2}-\omega_{m}^{2}\right) \theta(-\omega)=\delta\left(\omega+\omega_{m}\right)
\end{gathered}
$$

(The delta function derivation assumes $\omega_{m}>0$ see appendix) and

$$
[A]_{n_{1} n_{2}}\left(\omega^{2}\right)=2 \pi \sum_{m} e_{n_{1}}^{m} \delta\left(\omega^{2}-\omega_{m}^{2}\right)\left(e_{n_{2}}^{m}\right)^{*}
$$




$$
\begin{aligned}
{\left[G_{r_{i}}^{n}(\omega)\right]_{n_{1}, n_{2}} } & =\left[i D_{0}^{<}(\omega)\right]_{n_{1} n_{2}} \\
& =\sum_{m} \frac{2 \pi}{2 \omega_{m}}\left(e_{n_{2}}^{m}\right)^{*} e_{n_{1}}^{m}\left[\left(N_{-m}+1\right) \delta\left(\omega+\omega_{m}\right)+N_{m} \delta\left(\omega-\omega_{m}\right)\right] \\
& =\sum_{m} \frac{2 \pi}{2 \omega_{m}}\left(e_{n_{2}}^{m}\right)^{*} e_{n_{1}}^{m} 2 \omega_{m} \delta\left(\omega^{2}-\omega_{m}^{2}\right)\left[\left(N_{-m}+1\right) \theta(-\omega)+N_{m} \theta(\omega)\right] \\
& =\left[A\left(\omega^{2}\right)\right]_{n_{1} n_{2}}\left[\left(N_{-\omega}+1\right) \Theta(-\omega)+N_{\omega} \Theta(\omega)\right]
\end{aligned}
$$

\section{B.2.1 $G_{p}$ or $i D^{>}$}

$$
\begin{aligned}
{\left[G_{r_{i}}^{p}\left(t_{1}, t_{2}\right)\right]_{n_{1}, n_{2}} } & =\left[i D_{0}^{>}\left(t_{1}, t_{2}\right)\right]_{n_{1}, n_{2}} \\
{\left[G_{r_{i}}^{p}(\tau)\right]_{n_{1}, n_{2}} } & =\left[i D_{0}^{>}\left(t_{1}-t_{2}\right)\right]_{n_{1} n_{2}}=\left[i D_{0}^{>}(\tau)\right]_{n_{1} n_{2}} \\
& =\frac{1}{\hbar}\left\langle x_{n_{1}}\left(t_{1}\right) x_{n_{2}}\left(t_{2}\right)\right\rangle \\
& =\sum_{m_{1} m_{2}} \frac{1}{2 \sqrt{\omega_{m_{1}} \omega_{m_{2}}}}\left[\left\langle a_{m_{1}} a_{m_{2}}^{\dagger}\right\rangle e_{n_{1}}^{m_{1}}\left(e_{n_{2}}^{m_{2}}\right)^{*} e^{i\left(\omega_{m_{2}} t_{2}-\omega_{m_{1}} t_{1}\right)}+\left\langle a_{m_{1}}^{\dagger} a_{m_{2}}\right\rangle\left(e_{n_{1}}^{m_{1}}\right)^{*} e_{n_{2}}^{m_{2}} e^{i\left(\omega_{m_{1}} t_{1}-\omega_{\left.m_{2} t_{2}\right)}\right]}\right. \\
& =\sum_{m_{1} m_{2}} \frac{1}{2 \sqrt{\omega_{m_{1}} \omega_{m_{2}}}}\left[\left(N_{m_{1}}+1\right) \delta_{m_{1}, m_{2}} e_{n_{1}}^{m_{1}}\left(e_{n_{2}}^{m_{2}}\right)^{*} e^{i\left(\omega_{m_{2}} t_{2}-\omega_{m_{1}} t_{1}\right)}+N_{m_{1}} \delta_{m_{1}, m_{2}}\left(e_{n_{1}}^{m_{1}}\right)^{*} e_{n_{2}}^{m_{2}} e^{i\left(\omega_{m_{1}} t_{1}-\omega_{m_{2}} t_{2}\right)}\right] \\
& =\sum_{m} \frac{1}{2 \omega_{m}}\left[\left(N_{m}+1\right) e_{n_{1}}^{m}\left(e_{n_{2}}^{m}\right)^{*} e^{i \omega_{m}\left(t_{2}-t_{1}\right)}+N_{m}\left(e_{n_{1}}^{m}\right)^{*} e_{n_{2}}^{m} e^{i \omega_{m}\left(t_{1}-t_{2}\right)}\right] \\
& =\sum_{m} \frac{1}{2 \omega_{m}}\left[\left(N_{m}+1\right) e_{n_{1}}^{m}\left(e_{n_{2}}^{m}\right)^{*} e^{i \omega_{m}\left(t_{2}-t_{1}\right)}+N_{-m}\left(e_{n_{1}}^{-m}\right)^{*} e_{n_{2}}^{-m} e^{i \omega_{-m}\left(t_{1}-t_{2}\right)}\right] \\
& =\sum_{m} \frac{1}{2 \omega_{m}}\left[\left(N_{m}+1\right) e_{n_{1}}^{m}\left(e_{n_{2}}^{m}\right)^{*} e^{-i \omega_{m} \tau}+N_{-m} e_{n_{1}}^{m}\left(e_{n_{2}}^{m}\right)^{*} e^{i \omega_{m} \tau}\right] \\
& =\sum_{m} \frac{1}{2 \omega_{m}}\left(e_{n_{2}}^{m}\right)^{*} e_{n_{1}}^{m}\left[\left(N_{m}+1\right) e^{-i \omega_{m} \tau}+N_{-m} e^{i \omega_{m} \tau}\right]
\end{aligned}
$$

$$
\begin{aligned}
{\left[G_{r_{i}}^{p}(\omega)\right]_{n_{1}, n_{2}} } & =\left[i D_{0}^{>}(\omega)\right]_{n_{1} n_{2}} \\
& =\sum_{m} \frac{2 \pi}{2 \omega_{m}}\left(e_{n_{2}}^{m}\right)^{*} e_{n_{1}}^{m}\left[\left(N_{m}+1\right) \delta\left(\omega-\omega_{m}\right)+N_{-m} \delta\left(\omega+\omega_{m}\right)\right] \\
& =\sum_{m} \frac{2 \pi}{2 \omega_{m}}\left(e_{n_{2}}^{m}\right)^{*} e_{n_{1}}^{m} 2 \omega_{m} \delta\left(\omega^{2}-\omega_{m}^{2}\right)\left[\left(N_{m}+1\right) \theta(\omega)+N_{-m} \theta(-\omega)\right] \\
& =\left[A\left(\omega^{2}\right)\right]_{n_{1} n_{2}}\left[\left(N_{\omega}+1\right) \Theta(\omega)+N_{-\omega} \Theta(-\omega)\right]
\end{aligned}
$$


Note that in equation B.39 I could've replace $m$ by $-m$ on the other factor. That way we would've get

$$
\left[G_{r_{i}}^{p}(\tau)\right]_{n_{1}, n_{2}}=\sum_{m} \frac{1}{2 \omega_{m}}\left(e_{n_{1}}^{m}\right)^{*} e_{n_{2}}^{m}\left[\left(N_{-m}+1\right) e^{-i \omega_{m} \tau}+N_{m} e^{i \omega_{m} \tau}\right]
$$

which at the end of the process will lead to

$$
\left[G_{r_{i}}^{p}(\omega)\right]_{n_{1}, n_{2}}=\left[A\left(\omega^{2}\right)\right]_{n_{2} n_{1}}\left[\left(N_{-\omega}+1\right) \Theta(\omega)+N_{\omega} \Theta(-\omega)\right]
$$

Then we have the relationship

$$
G_{r}^{n}(-\omega)=G_{r}^{p}(\omega)=\left[G_{r}^{p}(\omega)\right]^{T}
$$

\section{B.2.2 Retarded Green's Function}

Now let's do the retarded green's function

$$
\begin{aligned}
{\left[i G^{R}\left(t_{1}, t_{2}\right)\right]_{n_{1}, n_{2}} } & =\left[i D^{R}\left(t_{1}, t_{2}\right)\right]_{n_{1}, n_{2}} \\
{\left[i G^{R}\left(t_{1}-t_{2}\right)\right]_{n_{1}, n_{2}} } & =\left[i D^{R}\left(t_{1}-t_{2}\right)\right]_{n_{1}, n_{2}} \\
{\left[i G^{R}(\tau)\right]_{n_{1}, n_{2}} } & =\left[i D^{R}(\tau)\right]_{n_{1}, n_{2}} \\
= & \frac{1}{\hbar} \Theta(\tau)\left\langle\left[x_{n_{1}}\left(t_{1}\right), x_{n_{2}}\left(t_{2}\right)\right]\right\rangle \\
= & \frac{1}{\hbar} \Theta(\tau)\left[\left\langle x_{n_{1}}\left(t_{1}\right) x_{n_{2}}\left(t_{2}\right)\right\rangle-\left\langle x_{n_{2}}\left(t_{2}\right) x_{n_{1}}\left(t_{1}\right)\right\rangle\right] \\
= & \Theta(\tau)\left[\sum_{m} \frac{1}{2 \omega_{m}}\left(e_{n_{2}}^{m}\right)^{*} e_{n_{1}}^{m}\left[\left(N_{m}+1\right) e^{-i \omega_{m} \tau}+N_{-m} e^{i \omega_{m} \tau}-\left(N_{-m}+1\right) e^{i \omega_{m} \tau}-N_{m} e^{-i \omega_{m} \tau}\right]\right] \\
= & \Theta(\tau)\left[\sum_{m} \frac{1}{2 \omega_{m}}\left(e_{n_{2}}^{m}\right)^{*} e_{n_{1}}^{m}\left[e^{-i \omega_{m} \tau}-e^{i \omega_{m} \tau}\right]\right]
\end{aligned}
$$

Now fourier transforming using

$$
\begin{aligned}
& F^{-1}\left\{\frac{1}{\omega-\omega_{m}+i \eta}\right\}=-i \theta(t) e^{-i \omega_{m} t} \\
& F^{-1}\left\{\frac{1}{\omega+\omega_{m}+i \eta}\right\}=-i \theta(t) e^{i \omega_{m} t}
\end{aligned}
$$




$$
\begin{aligned}
{\left[G^{R}(\omega)\right]_{n_{1}, n_{2}} } & =\left[D^{R}(\omega)\right]_{n_{1}, n_{2}} \\
& =\sum_{m} \frac{1}{2 \omega_{m}}\left(e_{n_{2}}^{m}\right)^{*} e_{n_{1}}^{m}\left[\frac{1}{\omega-\omega_{m}+i \eta}-\frac{1}{\omega+\omega_{m}+i \eta}\right] \\
& =\sum_{m} \frac{1}{2 \omega_{m}}\left(e_{n_{2}}^{m}\right)^{*} e_{n_{1}}^{m}\left[\frac{2 \omega_{m}}{(\omega+i \eta)^{2}-\omega_{m}^{2}}\right] \\
& =\sum_{m}\left[\frac{\left(e_{n_{2}}^{m}\right)^{*} e_{n_{1}}^{m}}{(\omega+i \eta)^{2}-\omega_{m}^{2}}\right] \\
& =G
\end{aligned}
$$

Note that there is a suttle difference in the definition of $\eta$ between this $G$ and the one we used (negleting $\eta^{2}$ and defining $\left.\eta^{\prime}=2 \omega \eta\right)$

$$
\begin{aligned}
G_{n_{1} n_{2}} & =\sum_{m} e_{n_{1}}^{m}\left[\frac{1}{(\omega+i \eta)^{2}-\omega_{m}^{2}}\right]\left(e_{n_{2}}^{m}\right)^{*} \\
& =\sum_{m} e_{n_{1}}^{m}\left[\frac{1}{\left(\omega^{2}+i 2 \omega \eta-\eta^{2}\right)-\omega_{m}^{2}}\right]\left(e_{n_{2}}^{m}\right)^{*} \\
& =\sum_{m} e_{n_{1}}^{m}\left[\frac{1}{\omega^{2}+i \eta^{\prime}-\omega_{m}^{2}}\right]\left(e_{n_{2}}^{m}\right)^{*} \\
& =G_{n_{1} n_{2}} \text { (what we use) }
\end{aligned}
$$

\section{B.3 Average Harmonic Heat Current}

$$
I_{r_{i}}=\int_{0}^{\infty} \frac{d \omega}{2 \pi} \hbar \omega \operatorname{Tr}\left\{\Sigma_{r_{i}}^{i n} A-G^{n} \Gamma_{r_{i}}\right\}
$$

Now (last steps because I'm only using it on positive frequencies)

$$
\begin{aligned}
\Sigma_{r_{i}}^{i n} & =\frac{1}{\hbar}\left\langle S_{r_{i}} S_{r_{i}}^{\dagger}\right\rangle \\
& =\frac{1}{\hbar}\left\langle\tau_{r_{i}} \boldsymbol{x}_{r_{i}} \boldsymbol{x}_{r_{i}}^{\dagger} \tau_{r_{i}}^{\dagger}\right\rangle \\
& =\tau_{r_{i}} \frac{1}{\hbar}\left\langle\boldsymbol{x}_{r_{i}} \boldsymbol{x}_{r_{i}}^{\dagger}\right\rangle \tau_{r_{i}}^{\dagger} \\
& =\tau_{r_{i}} G_{r_{i}}^{n} \tau_{r_{i}}^{\dagger} \\
& =\tau_{r_{i}} A_{r_{i}}\left(\omega^{2}\right) \tau_{r_{i}}^{\dagger}\left[\left(N_{-\omega}+1\right) \Theta(-\omega)+N_{\omega} \Theta(\omega)\right] \\
& =\Gamma_{r_{i}}(-\omega)\left(N_{-\omega}+1\right) \Theta(-\omega)+\Gamma_{r_{i}}(\omega) N_{\omega} \Theta(\omega)
\end{aligned}
$$




$$
\begin{aligned}
G^{n} & =\frac{1}{\hbar}\left\langle\boldsymbol{x} \boldsymbol{x}^{\dagger}\right\rangle \\
& =\frac{1}{\hbar}\left\langle G S S^{\dagger} G^{\dagger}\right\rangle \\
& =G\left\langle\frac{1}{\hbar} S S^{\dagger}\right\rangle G^{\dagger} \\
& =G\left\langle\sum_{r_{i}} \frac{1}{\hbar} S_{r_{i}} S_{r_{i}}^{\dagger}\right\rangle G^{\dagger} \\
& =G\left[\sum_{r_{i}} \Sigma_{r_{i}}^{i n}\right] G^{\dagger} \\
& =G \Sigma^{i n} G^{\dagger}
\end{aligned}
$$

\section{B.4 Conversion table}

Conversion table. I should define in the correlations in terms of field operators to compare properly

\begin{tabular}{|c|c|c|c|}
\hline Original & Datta (el) & Mingo & our \\
\hline$i G^{<}$ & $-G^{n}$ & $i D^{<}$ & $G^{n}$ \\
\hline$i G^{>}$ & $G^{p}$ & $i D^{>}$ & $G^{p}$ \\
\hline$i \Sigma^{<}$ & $-\Sigma^{i n}$ & $i \Sigma^{<}$ & $\Sigma^{i n}$ \\
\hline$i \Sigma^{>}$ & $\Sigma^{\text {out }}$ & $i \Sigma^{>}$ & $\Sigma^{\text {out }}$ \\
\hline$G^{R}$ & $G$ & $D^{R}=G$ & $G$ \\
\hline$G^{A}$ & $G^{\dagger}$ & $D^{A}=G^{\dagger}$ & $G^{\dagger}$ \\
\hline$\Sigma^{R}$ & $\Sigma$ & $\Sigma=\Sigma^{R}$ & $\Sigma$ \\
\hline$\Sigma^{A}$ & $\Sigma^{\dagger}$ & $\Sigma^{\dagger}$ & $\Sigma^{\dagger}$ \\
\hline
\end{tabular}

\section{B.4.1 Solution Procedure for Anharmonic NEGF}

1. Assume you know the contact details $M_{r_{i}}, K_{r_{i}}, T_{r_{i}} \rightarrow N_{r_{i}}$ and the channel details $M, K$ and the coupling details $\tau_{r_{i}}$. Also assume the following relations

$$
\begin{gathered}
G(-\omega)=G^{*}(\omega) \\
\Sigma(-\omega)=\Sigma^{*}(\omega) \\
\Gamma(-\omega)=-\Gamma^{*}(\omega) \\
\Sigma^{\text {in }}(-\omega)=\left[\Sigma^{\text {out }}(\omega)\right]^{T}
\end{gathered}
$$




$$
G^{n}(-\omega)=\left[G^{p}(\omega)\right]^{T}
$$

2. Calculate $\Sigma_{r_{1}}$ and $\Sigma_{r_{2}}$ from equation 2.35

$$
\Sigma_{r_{i}}=\tau_{r_{i}} G_{r_{i}} \tau_{r_{i}}^{\dagger}
$$

3. Calculate $\Gamma_{r 1}$ and $\Gamma_{r 2}$ from equation 2.41

$$
\Gamma_{r_{i}}=i\left(\Sigma_{r_{i}}-\Sigma_{r_{i}}^{\dagger}\right)
$$

4. Calculate $\Sigma_{r_{1}}^{i n}$ and $\Sigma_{r_{2}}^{i n}$ from equation B.67

$$
\sum_{r_{i}}^{i n}(\omega)=\Gamma_{r_{i}}(-\omega)\left(N_{r_{i}}(-\omega)+1\right) \Theta(-\omega)+\Gamma_{r_{i}}(\omega) N_{r_{i}}(\omega) \Theta(\omega)
$$

5. Calculate $\Sigma_{r_{1}}^{\text {out }}$ and $\Sigma_{r_{2}}^{\text {out }}$ from equation

$$
\begin{gathered}
\Sigma_{r_{i}}^{\text {out }}(\omega)=\Sigma_{r_{i}}^{\text {in }}(-\omega) \\
\sum_{r_{i}}^{\text {out }}(\omega)=\Gamma_{r_{i}}(\omega)\left(N_{r_{i}}(\omega)+1\right) \Theta(\omega)-\Gamma_{r_{i}}(-\omega) N_{r_{i}}(-\omega) \Theta(-\omega)
\end{gathered}
$$

6. Assume $\Sigma_{s}^{i n}=0, \Sigma_{s}=0$

7. Calculate $\Sigma$ and $\Sigma^{i n}$ from equations

$$
\begin{gathered}
\Sigma=\Sigma_{r_{1}}+\Sigma_{r_{2}}+\Sigma_{s} \\
\Sigma^{\text {in }}=\Sigma_{r_{1}}^{\text {in }}+\Sigma_{r_{2}}^{\text {in }}+\Sigma_{s}^{\text {in }} \\
\Sigma^{\text {out }}=\Sigma_{r_{1}}^{\text {out }}+\Sigma_{r_{2}}^{\text {out }}+\Sigma_{s}^{\text {out }}
\end{gathered}
$$

8. Calculate $G, G^{n}$ and $G^{p}$ using equation 2.34 and B.73

$$
\begin{gathered}
G=\left[\omega^{2} M-K-\Sigma\right]^{-1} \\
G^{n}=G \Sigma^{i n} G^{\dagger} \\
G^{p}=G \Sigma^{\text {out }} G^{\dagger}
\end{gathered}
$$


9. Calculate $\Sigma_{s}^{i n}$ and $\Sigma_{s}^{o u t}$. For three phonon processes

$$
\begin{gathered}
{\left[\Sigma_{s 1}^{i n}(\omega)\right]_{i, n}=-\hbar \sum_{j k l m} \int_{-\infty}^{\infty} d \omega^{\prime} V_{i j k} G_{j l}^{n}\left(\omega^{\prime}\right) G_{k m}^{n}\left(\omega-\omega^{\prime}\right) V_{l m n}} \\
{\left[\Sigma_{s 1}^{o u t}(\omega)\right]_{n, i}=-\hbar \sum_{j k l m} \int_{-\infty}^{\infty} d \omega^{\prime} V_{i j k} G_{j l}^{n}\left(\omega^{\prime}\right) G_{k m}^{n}\left(-\omega-\omega^{\prime}\right) V_{l m n}} \\
{\left[\Sigma_{s 2}^{i n}(\omega)\right]_{i, n}=-\hbar \sum_{j k l m} \int_{-\infty}^{\infty} d \omega^{\prime} V_{i j k} G_{j l}^{p}\left(\omega^{\prime}\right) G_{k m}^{n}\left(\omega+\omega^{\prime}\right) V_{l m n}} \\
{\left[\Sigma_{s 2}^{o u t}(\omega)\right]_{n, i}=-\hbar \sum_{j k l m} \int_{-\infty}^{\infty} d \omega^{\prime} V_{i j k} G_{j l}^{n}\left(\omega^{\prime}\right) G_{k m}^{p}\left(-\omega+\omega^{\prime}\right) V_{l m n}}
\end{gathered}
$$

10. Calculate $\Gamma_{s}$ from

$$
\Gamma_{s}=\Sigma_{s}^{o u t}-\Sigma_{s}^{i n}
$$

11. Calculate $\Im\left\{\Sigma_{s}\right\}$

$$
\Im\left\{\Sigma_{s}\right\}=-\frac{\Gamma_{s}}{2}
$$

12. Calculate $\Re\left\{\Sigma_{s}\right\}$ from the Hilbert transform

$$
\Re\left\{\Sigma_{s}\right\}=-\frac{1}{\pi} \int d \omega^{\prime} \frac{\Im\left\{\Sigma_{s}\right\}\left(\omega^{\prime}\right)}{\omega-\omega^{\prime}}=\frac{1}{2 \pi} \int d \omega^{\prime} \frac{\Gamma_{s}\left(\omega^{\prime}\right)}{\omega-\omega^{\prime}}
$$

13. With $\Sigma_{s}$ and $\Sigma_{s}^{i n}$ recalculate $\Sigma$ and $\Sigma^{i n}($ step 7$)$

$$
\begin{gathered}
\Sigma=\Sigma_{r_{1}}+\Sigma_{r_{2}}+\Sigma_{s} \\
\Sigma^{\text {in }}=\Sigma_{r_{1}}^{\text {in }}+\Sigma_{r_{2}}^{\text {in }}+\Sigma_{s}^{\text {in }} \\
\Sigma^{\text {out }}=\Sigma_{r_{1}}^{\text {out }}+\Sigma_{r_{2}}^{\text {out }}+\Sigma_{s}^{\text {out }}
\end{gathered}
$$

\section{B.5 Some equalities and equations}

$$
i\left(\Sigma_{r}(\omega)-\Sigma_{r}^{*}(\omega)\right)= \begin{cases}\tau_{r} A_{r}(\omega) \tau_{r}^{\dagger} & \text { if } \omega>0 \\ -\tau_{r} A_{r}(\omega) \tau_{r}^{\dagger} & \text { if } \omega<0\end{cases}
$$




$$
\begin{gathered}
-2 \Im\left\{\Sigma_{r}(\omega)\right\}= \begin{cases}\tau_{r} A_{r}(\omega) \tau_{r}^{\dagger} & \text { if } \omega>0 \\
-\tau_{r} A_{r}(\omega) \tau_{r}^{\dagger} & \text { if } \omega<0\end{cases} \\
A_{r}(-\omega)=A_{r}(\omega) \\
A_{r}(\omega)=\left[A_{r}(\omega)\right]^{T} \\
\sum_{r}^{\text {in }}(\omega)=\tau_{r} A_{r}(\omega) \tau_{r}^{\dagger}\left[\Theta(-\omega)+N_{r}\left(\omega^{2}\right)\right] \\
\Sigma_{r}^{\text {out }}(\omega)=\tau_{r} A_{r}(\omega) \tau_{r}^{\dagger}\left[\Theta(\omega)+N_{r}\left(\omega^{2}\right)\right] \\
J_{h}(\omega)=\frac{\hbar \omega}{2 \pi} \operatorname{Tr}\left\{\Sigma_{h}^{\text {in }} G^{p}-\Sigma_{h}^{\text {out }} G^{n}\right\}
\end{gathered}
$$

\section{B.6 Delta function derivation}

Let's assume $\omega_{m}>0$ as it is the case

$$
\begin{aligned}
\delta\left(\omega^{2}-\omega_{m}^{2}\right) & =\frac{\delta\left(\omega-\omega_{m}\right)}{\left|2 \omega_{m}\right|}+\frac{\delta\left(\omega+\omega_{m}\right)}{\left|-2 \omega_{m}\right|} \\
& =\frac{1}{2 \omega_{m}}\left[\delta\left(\omega-\omega_{m}\right)+\delta\left(\omega+\omega_{m}\right)\right] \\
2 \omega_{m} \delta\left(\omega^{2}-\omega_{m}^{2}\right) & =\delta\left(\omega-\omega_{m}\right)+\delta\left(\omega+\omega_{m}\right)
\end{aligned}
$$




\section{Bibliography}

[1] E. Pop, S. Sinha, and Kenneth E. Goodson. Heat generation and transport in nanometer-scale transistors. Proceedings of the IEEE, 94(8):1587-1601, 2006.

[2] Patrick E. Hopkins. Thermal transport across solid interfaces with nanoscale imperfections: Effects of roughness, disorder, dislocations, and bonding on thermal boundary conductance. ISRN Mechanical Engineering, 2013(682586):19, 2013.

[3] Mark D. Losego, Martha E. Grady, Nancy R. Sottos, David G. Cahill, and Paul V. Braun. Effects of chemical bonding on heat transport across interfaces. Nat Mater, 11(6):502-506, Jun 2012.

[4] Maria N. Luckyanova, Jivtesh Garg, Keivan Esfarjani, Adam Jandl, Mayank T. Bulsara, Aaron J. Schmidt, Austin J. Minnich, Shuo Chen, Mildred S. Dresselhaus, Zhifeng Ren, Eugene A. Fitzgerald, and Gang Chen. Coherent phonon heat conduction in superlattices. Science, 338(6109):936-939, 2012.

[5] Changwook Jeong, Supriyo Datta, and Mark Lundstrom. Thermal conductivity of bulk and thin-film silicon: A landauer approach. Journal of Applied Physics, 111(9):093708, 2012.

[6] Carlos A. Polanco, Christopher B. Saltonstall, Pamela M. Norris, Patrick E. Hopkins, and Avik W. Ghosh. Impedance matching of atomic thermal interfaces using primitive block decomposition. Nanoscale and Microscale Thermophysical Engineering, 17(3):263-279, 2013.

[7] W. Zhang, T. S. Fisher, and N. Mingo. Simulation of interfacial phonon transport in si-ge heterostructures using an atomistic green's function method. Journal of Heat Transfer, 129(4):483-491, 2007.

[8] M. Holt, Z. Wu, Hawoong Hong, P. Zschack, P. Jemian, J. Tischler, Haydn Chen, and T.-C. Chiang. Determination of phonon dispersions from x-ray transmission scattering: The example of silicon. Phys. Rev. Lett., 83(16):3317-3319, Oct 1999.

[9] David G. Cahill, Wayne K. Ford, Kenneth E. Goodson, Gerald D. Mahan, Arun Majumdar, Humphrey J. Maris, Roberto Merlin, and Simon R. Phillpot. Nanoscale thermal transport. Journal of Applied Physics, 93(2):793-818, 2003.

[10] Eric Pop. Energy dissipation and transport in nanoscale devices. Nano Research, 3:147-169, 2010. 10.1007/s12274-010-1019-z.

[11] Eric S. Toberer, Lauryn L. Baranowski, and Chris Dames. Advances in thermal conductivity. Annual Review of Materials Research, 42(1):179-209, 2012.

[12] W. Haensch, , E.J. Nowak, R.H. Dennard, P.M. Solomon, A. Bryant, O.H. Dokumaci, A. Kumar, X. Wang, J.B. Johnson, and M.V. Fischetti. Silicon cmos devices beyond scaling. IBM Journal of Research and Development, 50(4.5):339-361, 2006.

[13] D. Vasileska, K. Raleva, and S.M. Goodnick. Modeling heating effects in nanoscale devices: thepresentandthefuture. Journal of Computational Electronics, 7(2):66-93, 2008.

[14] Aleksandr Chernatynskiy, David Clarke, and Simon Phillpot. Handbook of Nanoscience, Engineering, and Technology. Chapter 19: Thermal Transport in Nanostructured Materials. CRC Press, thrid edition, 2012. 
[15] Mark D. Losego and David G. Cahill. Thermal transport: Breaking through barriers. Nat Mater, page 2, 32013 .

[16] David G. Cahill, Paul V. Braun, Gang Chen, David R. Clarke, Shanhui Fan, Kenneth E. Goodson, Pawel Keblinski, William P. King, Gerald D. Mahan, Arun Majumdar, Humphrey J. Maris, Simon R. Phillpot, Eric Pop, and Li Shi. Nanoscale thermal transport. ii. 20032012. Applied Physics Reviews, 1(1):-, 2014.

[17] Patrick E. Hopkins, Pamela M. Norris, Robert J. Stevens, Thomas E. Beechem, and Samuel Graham. Influence of interfacial mixing on thermal boundary conductance across a chromium/silicon interface. Journal of Heat Transfer, 130(6):062402, 2008.

[18] Jayakanth Ravichandran, Ajay K. Yadav, Ramez Cheaito, Pim B. Rossen, Arsen Soukiassian, S. J. Suresha, John C. Duda, Brian M. Foley, Che-Hui Lee, Ye Zhu, Arthur W. Lichtenberger, Joel E. Moore, David A. Muller, Darrell G. Schlom, Patrick E. Hopkins, Arun Majumdar, Ramamoorthy Ramesh, and Mark A. Zurbuchen. Crossover from incoherent to coherent phonon scattering in epitaxial oxide superlattices. Nat Mater, 13(2):168-172, February 2014.

[19] E. T. Swartz and R. O. Pohl. Thermal boundary resistance. Rev. Mod. Phys., 61(3):605-668, Jul 1989.

[20] Ravi Prasher. Acoustic mismatch model for thermal contact resistance of van der waals contacts. Applied Physics Letters, 94(4):041905, 2009.

[21] Patrick E. Hopkins, John C. Duda, and Pamela M. Norris. Anharmonic phonon interactions at interfaces and contributions to thermal boundary conductance. Journal of Heat Transfer, 133(6):11, 3 2011.

[22] John C. Duda, Thomas E. Beechem, Justin L. Smoyer, Pamela M. Norris, and Patrick E. Hopkins. Role of dispersion on phononic thermal boundary conductance. Journal of Applied Physics, 108(7):073515, 2010.

[23] John C. Duda, Patrick E. Hopkins, Justin L. Smoyer, Matthew L. Bauer, Timothy S. English, Christopher B. Saltonstall, and Pamela M. Norris. On the assumption of detailed balance in prediction of diffusive transmission probability during interfacial transport. Nanoscale and Microscale Thermophysical Engineering, 14(1):21-33, 2010.

[24] Supriyo Datta. Quantum Transport: Atom to Transistor. Cambridge University Press, 2nd edition, July 2005.

[25] Paolo Giannozzi, Stefano Baroni, Nicola Bonini, Matteo Calandra, Roberto Car, Carlo Cavazzoni, Davide Ceresoli, Guido L Chiarotti, Matteo Cococcioni, Ismaila Dabo, Andrea Dal Corso, Stefano de Gironcoli, Stefano Fabris, Guido Fratesi, Ralph Gebauer, Uwe Gerstmann, Christos Gougoussis, Anton Kokalj, Michele Lazzeri, Layla Martin-Samos, Nicola Marzari, Francesco Mauri, Riccardo Mazzarello, Stefano Paolini, Alfredo Pasquarello, Lorenzo Paulatto, Carlo Sbraccia, Sandro Scandolo, Gabriele Sclauzero, Ari P Seitsonen, Alexander Smogunov, Paolo Umari, and Renata M Wentzcovitch. Quantum espresso: a modular and open-source software project for quantum simulations of materials. Journal of Physics: Condensed Matter, 21(39):395502, 2009.

[26] Wu Li, Jesus Carrete, Nebil A. Katcho, and Natalio Mingo. Shengbte: a solver of the boltzmann transport equation for phonons. Comp. Phys. Commun., 185:17471758, 2014.

[27] N. Mingo and Liu Yang. Phonon transport in nanowires coated with an amorphous material: An atomistic green's function approach. Phys. Rev. B, 68:245406, Dec 2003.

[28] N. Mingo. Anharmonic phonon flow through molecular-sized junctions. Phys. Rev. B, 74:125402, Sep 2006.

[29] Jian-Sheng Wang, Jian Wang, and Nan Zeng. Nonequilibrium green's function approach to mesoscopic thermal transport. Phys. Rev. B, 74:033408, Jul 2006. 
[30] Takahiro Yamamoto and Kazuyuki Watanabe. Nonequilibrium green's function approach to phonon transport in defective carbon nanotubes. Phys. Rev. Lett., 96:255503, Jun 2006.

[31] J.-S. Wang, J. Wang, and J. T. L. Quantum thermal transport in nanostructures. The European Physical Journal B, 62(4):381-404, 2008.

[32] W. Zhang, N. Mingo, and T. S. Fisher. Simulation of phonon transport across a non-polar nanowire junction using an atomistic green's function method. Phys. Rev. B, 76:195429, Nov 2007.

[33] Jin-Wu Jiang and Jian-Sheng Wang. Manipulation of heat current by the interface between graphene and white graphene. EPL (Europhysics Letters), 96(1):16003, 2011.

[34] Xiaobo Li and Ronggui Yang. Size-dependent phonon transmission across dissimilar material interfaces. Journal of Physics: Condensed Matter, 24(15):155302, 2012.

[35] Xuean Zhao, Jing Li, T. C. Au Yeung, C. H. Kam, Qing-Hu Chen, and Chang Q. Sun. Phonon transport in atomic chains coupled by thermal contacts: The role of buffer layer. Journal of Applied Physics, 107(9):094312, 2010.

[36] Xiaobo Li and Ronggui Yang. Effect of lattice mismatch on phonon transmission and interface thermal conductance across dissimilar material interfaces. Phys. Rev. B, 86:054305, Aug 2012.

[37] Jing Li, Tin Cheung Au Yeung, and Chan Hin Kam. Phonon transport in nanowire with contacts: Size and doping. Journal of Applied Physics, 111(9):094308, 2012.

[38] N. Mingo, D. A. Stewart, D. A. Broido, and D. Srivastava. Phonon transmission through defects in carbon nanotubes from first principles. Phys. Rev. B, 77:033418, Jan 2008.

[39] Ivana Savić, Derek A. Stewart, and Natalio Mingo. Thermal conduction mechanisms in boron nitride nanotubes: Few-shell versus all-shell conduction. Phys. Rev. B, 78:235434, Dec 2008.

[40] Derek A. Stewart, Ivana Savic?, and Natalio Mingo. First-principles calculation of the isotope effect on boron nitride nanotube thermal conductivity. Nano Letters, 9(1):81-84, 2009.

[41] Zhen Huang, Timothy Fisher, and Jayathi Murthy. An atomistic study of thermal conductance across a metal-graphene nanoribbon interface. Journal of Applied Physics, 109(7):074305, 2011.

[42] A. Kundu, N. Mingo, D. A. Broido, and D. A. Stewart. Role of light and heavy embedded nanoparticles on the thermal conductivity of sige alloys. Phys. Rev. B, 84:125426, Sep 2011.

[43] Zhiting Tian, Keivan Esfarjani, and Gang Chen. Enhancing phonon transmission across a si/ge interface by atomic roughness: First-principles study with the green's function method. Phys. Rev. B, 86:235304, Dec 2012.

[44] M. Bachmann, M. Czerner, S. Edalati-Boostan, and C. Heiliger. Ab initio calculations of phonon transport in zno and zns. The European Physical Journal B, 85(5):1-9, 2012.

[45] M. Bachmann, M. Czerner, and C. Heiliger. Coherent phonon scattering in zno and zns at sulfite and oxygen impurities. physica status solidi (a), 210(1):125-130, 2013.

[46] Yong Xu, Jian-Sheng Wang, Wenhui Duan, Bing-Lin Gu, and Baowen Li. Nonequilibrium green's function method for phonon-phonon interactions and ballistic-diffusive thermal transport. Phys. Rev. $B, 78: 224303$, Dec 2008.

[47] Natalio Mingo. Green's function methods for phonon transport through nano-contacts. In Sebastian Volz, editor, Thermal Nanosystems and Nanomaterials, volume 118 of Topics in Applied Physics, pages 63-94. Springer Berlin Heidelberg, 2009.

[48] Jing Li, T. C. Au Yeung, C. H. Kam, Yue Peng, Qing hu Chen, Xuean Zhao, and Chang Q. Sun. Anharmonic phonon transport in atomic wire coupled by thermal contacts with surface bond reconstruction. Journal of Applied Physics, 106(1):014308, 2009. 
[49] Mathieu Luisier. Atomistic modeling of anharmonic phonon-phonon scattering in nanowires. Phys. Rev. $B, 86: 245407$, Dec 2012.

[50] Zhen Huang, Jayathi Y. Murthy, and Timothy S. Fisher. Modeling of polarization-specific phonon transmission through interfaces. Journal of Heat Transfer, 133(114502):3, Sep 2011.

[51] Gang Chen. Nanoscale Energy Transport and Conversion : A Parallel Treatment of Electrons, Molecules, Phonons, and Photons: A Parallel Treatment of Electrons, Molecules, Phonons, and Photons. MIT-Pappalardo series in mechanical engineering. Oxford University Press, USA, 2005.

[52] P. W. Anderson, D. J. Thouless, E. Abrahams, and D. S. Fisher. New method for a scaling theory of localization. Phys. Rev. B, 22:3519-3526, Oct 1980.

[53] S. Datta. Electronic Transport in Mesoscopic Systems. Cambridge Studies in Semiconductor Physics and Microelectronic Engineering. Cambridge University Press, 1997.

[54] Changwook Jeong, Supriyo Datta, and Mark Lundstrom. Full dispersion versus debye model evaluation of lattice thermal conductivity with a landauer approach. Journal of Applied Physics, 109(7):-, 2011.

[55] M. Asen-Palmer, K. Bartkowski, E. Gmelin, M. Cardona, A. P. Zhernov, A. V. Inyushkin, A. Taldenkov, V. I. Ozhogin, K. M. Itoh, and E. E. Haller. Thermal conductivity of germanium crystals with different isotopic compositions. Phys. Rev. B, 56:9431-9447, Oct 1997.

[56] Patrick E. Hopkins, Leslie M. Phinney, Justin R. Serrano, and Thomas E. Beechem. Effects of surface roughness and oxide layer on the thermal boundary conductance at aluminum/silicon interfaces. Phys. Rev. B, 82:085307, Aug 2010.

[57] Patrick E. Hopkins, Khalid Hattar, Thomas Beechem, Jon F. Ihlefeld, Douglas L. Medlin, and Edward S. Piekos. Reduction in thermal boundary conductance due to proton implantation in silicon and sapphire. Applied Physics Letters, 98(23):231901, 2011.

[58] Patrick E. Hopkins, John C. Duda, Stephen P. Clark, Christopher P. Hains, Thomas J. Rotter, Leslie M. Phinney, and Ganesh Balakrishnan. Effect of dislocation density on thermal boundary conductance across gasb/gaas interfaces. Applied Physics Letters, 98(16):161913, 2011.

[59] Patrick E. Hopkins, Thomas Beechem, John C. Duda, Khalid Hattar, Jon F. Ihlefeld, Mark A. Rodriguez, and Edward S. Piekos. Influence of anisotropy on thermal boundary conductance at solid interfaces. Phys. Rev. B, 84:125408, Sep 2011.

[60] John C. Duda and Patrick E. Hopkins. Systematically controlling kapitza conductance via chemical etching. Applied Physics Letters, 100(11):111602, 2012.

[61] Ming Hu, Pawel Keblinski, and Patrick K. Schelling. Kapitza conductance of silicon `amorphous polyethylene interfaces by molecular dynamics simulations. Phys. Rev. B, 79:104305, Mar 2009.

[62] Lin Hu, Lifa Zhang, Ming Hu, Jian-Sheng Wang, Baowen Li, and Pawel Keblinski. Phonon interference at self-assembled monolayer interfaces: Molecular dynamics simulations. Phys. Rev. B, 81:235427, Jun 2010.

[63] Meng Shen, William J. Evans, David Cahill, and Pawel Keblinski. Bonding and pressure-tunable interfacial thermal conductance. Phys. Rev. B, 84:195432, Nov 2011.

[64] Christopher B. Saltonstall, Carlos A. Polanco, John C. Duda, Avik W. Ghosh, Pamela M. Norris, and Patrick E. Hopkins. Effect of interface adhesion and impurity mass on phonon transport at atomic junctions. Journal of Applied Physics, 113(1):-, 2013.

[65] Lifa Zhang, Pawel Keblinski, Jian-Sheng Wang, and Baowen Li. Interfacial thermal transport in atomic junctions. Phys. Rev. B, 83(6):064303, Feb 2011.

[66] H. J. Pain. The physics of vibrations and waves. Wiley, 2nd edition, 1976. 
[67] Patrick E. Hopkins, Pamela M. Norris, Mikiyas S. Tsegaye, and Avik W. Ghosh. Extracting phonon thermal conductance across atomic junctions: Nonequilibrium green's function approach compared to semiclassical methods. Journal of Applied Physics, 106(6):063503, 2009.

[68] W. Zhang, T. S. Fisher, and N. Mingo. The atomistic green's function method: An efficient simulation approach for nanoscale phonon transport. Numerical Heat Transfer, Part B, 51(4):333-349, 2007.

[69] Neil W. Ashcroft and David N. Mermin. Solid State Physics. Thomson Learning, Toronto, 1 edition, January 1976.

[70] Fernando de León-Pérez and Rolando Pérez-Alvarez. Phonon propagation in nonpolar semiconductor heterostructures. Phys. Rev. B, 63:245304, May 2001.

[71] de León-Pérez Fernando Villegas, Diosdado. and Rolando Pérez-Alvarez. Longwave phonon tunnelling using an impedance concept. PIERS ONLINE, 4:227-230, 2008.

[72] David M. Pozar. Microwave Engineering. Wiley, 4 edition, November 2011.

[73] A. G. Kozorezov, J. K. Wigmore, C. Erd, A. Peacock, and A. Poelaert. Scattering-mediated transmission and reflection of high-frequency phonons at a nonideal solid-solid interface. Phys. Rev. B, 57:7411-7414, Apr 1998.

[74] G. Fagas, A. G. Kozorezov, C. J. Lambert, J. K. Wigmore, A. Peacock, A. Poelaert, and R. den Hartog. Lattice dynamics of a disordered solid-solid interface. Phys. Rev. B, 60:6459-6464, Sep 1999.

[75] Hong Zhao and Jonathan B. Freund. Phonon scattering at a rough interface between two fcc lattices. Journal of Applied Physics, 105(1):-, 2009.

[76] H. Zhao and J. B. Freund. Lattice-dynamical calculation of phonon scattering at ideal sige interfaces. Journal of Applied Physics, 97(2):-, 2005.

[77] D Kechrakos. The phonon boundary scattering cross section at disordered crystalline interfaces: a simple model. Journal of Physics: Condensed Matter, 2(11):2637, 1990.

[78] Ashton Skye and Patrick K. Schelling. Thermal resistivity of si-ge alloys by molecular-dynamics simulation. Journal of Applied Physics, 103(11):-, 2008.

[79] Takuru Murakami, Takuma Shiga, Takuma Hori, Keivan Esfarjani, and Junichiro Shiomi. Importance of local force fields on lattice thermal conductivity reduction in pbte ${ }_{1-x} \mathrm{se}_{x}$ alloys. EPL (Europhysics Letters), 102(4):46002, 2013.

[80] Robert J. Stevens, Leonid V. Zhigilei, and Pamela M. Norris. Effects of temperature and disorder on thermal boundary conductance at solid-solid interfaces: Nonequilibrium molecular dynamics simulations. International Journal of Heat and Mass Transfer, 50(19-20):3977 - 3989, 2007.

[81] E. S. Landry and A. J. H. McGaughey. Thermal boundary resistance predictions from molecular dynamics simulations and theoretical calculations. Phys. Rev. B, 80:165304, Oct 2009.

[82] Carlos A. Polanco and Avik W. Ghosh. Enhancing phonon flow through one-dimensional interfaces by impedance matching. Journal of Applied Physics, 116(8):-, 2014.

[83] P. K. Schelling, S. R. Phillpot, and P. Keblinski. Phonon wave-packet dynamics at semiconductor interfaces by molecular-dynamics simulation. Applied Physics Letters, 80(14):2484-2486, 2002.

[84] A. J. H. McGaughey, M. I. Hussein, E. S. Landry, M. Kaviany, and G. M. Hulbert. Phonon band structure and thermal transport correlation in a layered diatomic crystal. Phys. Rev. B, 74:104304, Sep 2006. 
[85] Timothy S. English, John C. Duda, Justin L. Smoyer, Donald A. Jordan, Pamela M. Norris, and Leonid V. Zhigilei. Enhancing and tuning phonon transport at vibrationally mismatched solid-solid interfaces. Phys. Rev. B, 85:035438, Jan 2012.

[86] J. P. Perdew and Alex Zunger. Self-interaction correction to density-functional approximations for many-electron systems. Phys. Rev. B, 23:5048-5079, May 1981.

[87] Timothy B. Boykin, Neerav Kharche, and Gerhard Klimeck. Brillouin-zone unfolding of perfect supercells having nonequivalent primitive cells illustrated with a $\mathrm{Si} / \mathrm{Ge}$ tight-binding parameterization. Phys. Rev. B, 76:035310, Jul 2007.

[88] Frank H. Stillinger and Thomas A. Weber. Computer simulation of local order in condensed phases of silicon. Phys. Rev. B, 31:5262-5271, Apr 1985.

[89] Michael Galperin, Sivan Toledo, and Abraham Nitzan. Numerical computation of tunneling fluxes. The Journal of Chemical Physics, 117(23):10817-10826, 2002.

[90] W.A. Harrison. Electronic structure and the properties of solids: the physics of the chemical bond. Dover books on physics. Dover Publications, 1989.

[91] David G. Cahill. Extremes of heat conduction: Pushing the boundaries of the thermal conductivity of materials. MRS Bulletin, 37:855-863, 82012.

[92] Patrick E. Hopkins, Mira Baraket, Edward V. Barnat, Thomas E. Beechem, Sean P. Kearney, John C. Duda, Jeremy T. Robinson, and Scott G. Walton. Manipulating thermal conductance at metalgraphene contacts via chemical functionalization. Nano Letters, 12(2):590-595, 2012.

[93] D Kechrakos. The role of interface disorder in the thermal boundary conductivity between two crystals. Journal of Physics: Condensed Matter, 3(11):1443, 1991.

[94] Yu-Shiang Lin, D Sylvester, and D Blaauw. A sub-pw timer using gate leakage for ultra low-power sub-hz monitoring systems. In Custom Integrated Circuits Conference, 200\%. CICC 'O\%. IEEE, pages 397-400, 2007.

[95] Chuck Moore. Data processing in exascale-class computer systems. www.lanl.gov/orgs/hpc/salishan/salishan2011/3moore.pdf, April 2011.

[96] Carlos A. Polanco, Rouzbeh Rastgarkafshgarkolaei, Jingjie Zhang, Nam Q. Le, Pamela M. Norris, Patrick E. Hopkins, and Avik W. Ghosh. Role of crystal structure and junction morphology on interface thermal conductance. Phys. Rev. B, 92:144302, Oct 2015.

[97] Mohsen Radfar, Jugdutt Singh, and Jugdutt Singh. Recent subthreshold design techniques. Active and Passive Electronic Components, 2012:11, 2012. 